\title{
Interpretation and Analysis on Various Time Scales of Narrow-Band Coronal Observations Obtained with a New Coronagraph System
}

\author{
Dissertation \\ zur Erlangung des Doktorgrades \\ der Mathematisch-Naturwissenschaftlichen Fakultäten \\ der Georg-August-Universität zu Göttingen
}

\author{
vorgelegt von \\ Guillermo Adrián Stenborg \\ aus \\ Buenos Aires/Argentinien
}

Göttingen 2000 
D7

Referent: Prof. Dr. Franz Kneer

Korreferent: Prof. Dr. Rainer Schwenn

Tag der mündlichen Prüfung: 21. Juni 2000 


\section{Contents}

$\begin{array}{ll}\text { Zusammenfassung } & 7\end{array}$

1 Introduction $\quad 9$

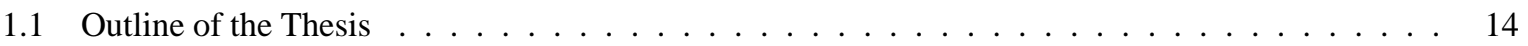

2 The Telescope and the Auxiliary Systems $\quad 17$

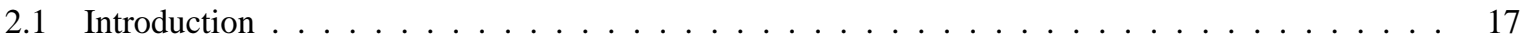

2.2 The optical layout of the MICA telescope . . . . . . . . . . . . . . . . . . . . . . 19

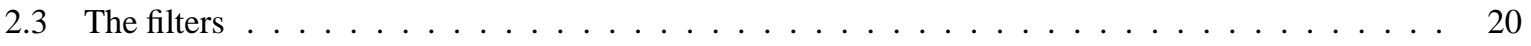

2.3.1 Theoretical background ......................... 21

2.3.2 The filters in MICA . . . . . . . . . . . . . . . . . . . . . 23

2.4 The CCD camera . . . . . . . . . . . . . . . . . . . . . . . . . 25

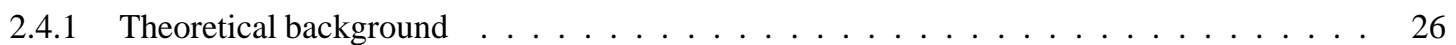

2.4 .2 The MICA camera . . . . . . . . . . . . . . . . . . . . . . . . . . . . . 29

2.5 Auxiliary devices . . . . . . . . . . . . . . . . . . . . . . . 31

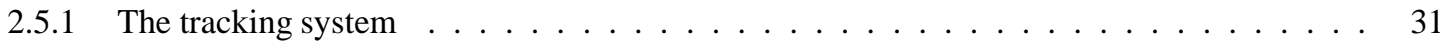

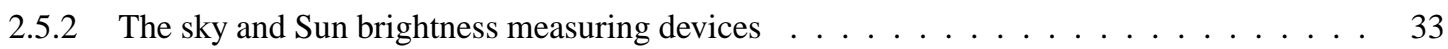

2.5.3 Temperature controlling . . . . . . . . . . . . . . . . . . 34

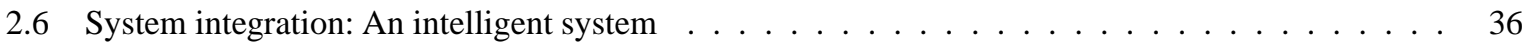

2.6 .1 Block diagram . . . . . . . . . . . . . . . . . . . . 36

2.6.2 How does the system work? . . . . . . . . . . . . . . . . . . 38

2.6 .3 The Graphical User Interface $(\mathrm{GUI}) \ldots \ldots \ldots$. . . . . . . . . . . . . . 40

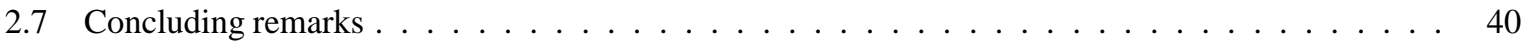

3 The Calibration of the Observations $\quad 43$

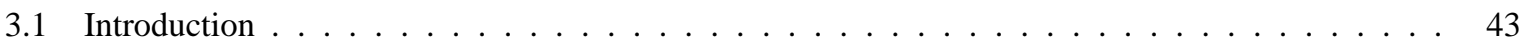

3.2 A theoretical view . . . . . . . . . . . . . . . . . . . . . 43

3.2 .1 Summary . . . . . . . . . . . . . . . . . . . 48

3.3 Practical implementation of the reduction and calibration procedure of MICA images . . . . . 51

3.3.1 On the development of the basic observation sequence . . . . . . . . . . . . 51

3.3 .2 On the determination of the calibration factor $\xi \ldots \ldots \ldots \ldots$

3.3.3 On the conversion of the on- and off-line images to relative units and continuum removal . 53

3.3 .4 On the determination of the scaling factor $\beta \ldots \ldots \ldots \ldots$

3.3.5 On the effects of the transmittance of the Earth's atmosphere $\tau_{i} \ldots \ldots \ldots$ 
3.3.6 $\bar{\beta}$ as an estimate of the accuracy of the calibration in regard to the transmittance of the Earth's atmosphere . . . . . . . . . . . . . . . . . . 60

3.3.7 On the determination of the normalization factor $f_{k} \ldots \ldots \ldots \ldots$

3.4 The practical real-time reduction scheme . . . . . . . . . . . . . . . . . . 62

3.5 Comparison with calibrated data . . . . . . . . . . . . . . . . . . . . 64

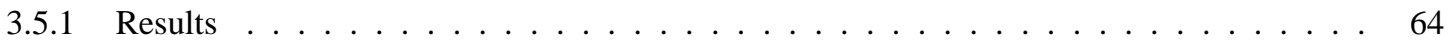

3.6 Concluding remarks on the calibration $\ldots \ldots \ldots \ldots \ldots \ldots$

3.6 .1 Constraints to the calibration . . . . . . . . . . . . . . . . . 65

3.6.2 On the interpretation of coronal images . . . . . . . . . . . . . . . . 68

4 The Observations at Short Time Scales $r$

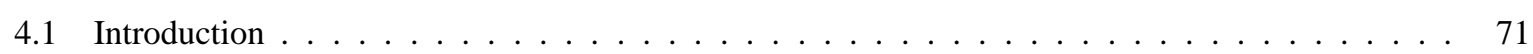

4.2 Mathematical description of typical scenarios observed in coronal images . . . . . . . . . . . 74

4.3 Stationary coronal features . . . . . . . . . . . . . . . . . . . 77

4.3.1 The green line above quiescent prominences _. . . . . . . . . . . . . . . 77

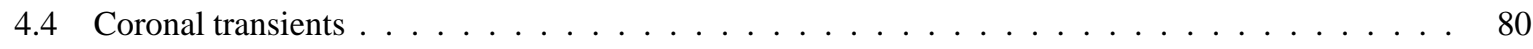

4.4.1 The September 30, 1998 event . . . . . . . . . . . . . . . . . . . 82

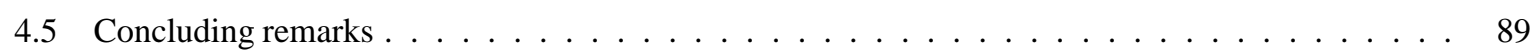

5 On the Rotation of the Emission Solar Corona 91

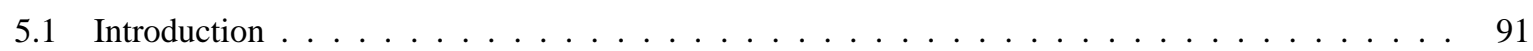

5.2 Part I: Analysis of the recurrence of the green line emission . . . . . . . . . . . . . . 93

5.2 .1 The LASCO-C1 observations . . . . . . . . . . . . . . . . . 93

5.2.2 The Phase Dispersion Minimization technique: a brief overview . . . . . . . . . . . 95

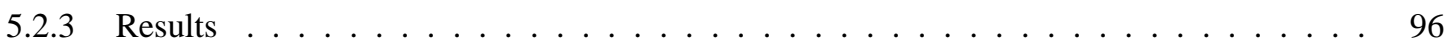

5.2 .4 Discussion ........................... 100

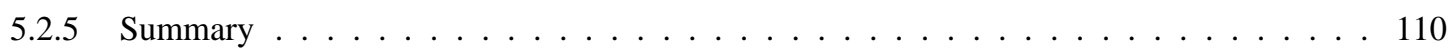

5.3 Part II: Analysis of the longitude displacement of coronal and transition-region features . . . . . 113

5.3 .1 The EIT observations . . . . . . . . . . . . . . . . . . . . . 113

5.3.2 The method to compute the longitude displacement . . . . . . . . . . . . . . 115

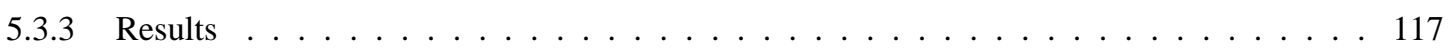

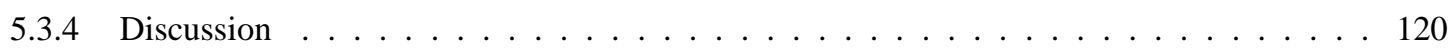

5.4 Concluding remarks . . . . . . . . . . . . . . . . . . . . . . . . 122

6 Summary and Outlook $r$

6.1 Future prospects . . . . . . . . . . . . . . . . . . . . . 127

A On the Spectral Distribution of the Sunlight 129 
B On the Atmospheric Extinction $\quad 132$

B.1 Air mass . . . . . . . . . . . . . . . . . . . . . . . . . 132

B.2 Transmittance of the Earth's atmosphere . . . . . . . . . . . . . . . . . . . 133

B.2.1 Rayleigh scattering of air molecules . . . . . . . . . . . . . . . . 133

B.2.2 Mie scattering of water vapour and dust particles . . . . . . . . . . . . . . 133

B.2.3 Absorption by gases . . . . . . . . . . . . . . . . . . . 134

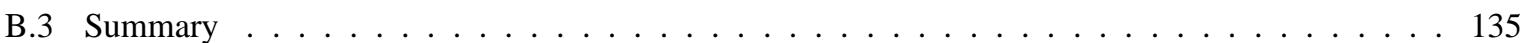

$\begin{array}{llr}\text { C The HASTA Instrument } & 136\end{array}$

$\begin{array}{lr}\text { List of Figures } & 137\end{array}$

$\begin{array}{lr}\text { List of Tables } & 139\end{array}$

$\begin{array}{lr}\text { Bibliography } & 141\end{array}$

$\begin{array}{lr}\text { Danksagung } & 149\end{array}$

$\begin{array}{lr}\text { Lebenslauf } & 151\end{array}$ 



\section{Zusammenfassung}

Die Analyse der Struktur des koronalen Plasmas gibt wichtige Einblicke in die Topologie des magnetischen Feldes in der Sonnenatmosphäre. Systematische Beobachtungen der Entwicklung von Strukturen der inneren Korona über längere Zeiträume sagen etwas aus sowohl über die globale solare Aktivität als auch über kleinskalige dynamische Vorgänge. Beobachtungen mit hoher zeitlicher und räumlicher Auflösung können die Randbedingungen für theoretische Modelle und auch Kriterien für deren Gültigkeit liefern.

Für die Durchführung solcher Beobachtungen war ein neuartiger Spiegelkoronograph mit internem Okkulter (MICA) am Max-Planck-Institut für Aeronomie in Katlenburg-Lindau (MPAe) entwickelt und im August des Jahres 1997 in El Leoncito, Argentinien aufgestellt worden. Im Rahmen dieser Arbeit wurde der Betrieb von MICA automatisiert, unter besonderer Berücksichtigung von externen Umgebungsbedingungen (z.B. Klarheit und Konstanz der Erdatmosphäre, Wolken und Wind) und geräteinternen Kriterien (z.B. Temperaturregelung von Struktur und Filtern, CCD-Kamera, Filter, Eingangstür, Nachführung usw.). Die Zuverlässigkeit der entwickelten Kalibrationsprozedur wurde ausführlich untersucht. Dabei spielt auch der Extinktionskoeffizient der Erdatmosphäre und insbesondere seine Variabilität eine entscheidende Rolle.

Die Interpretation der beobachteten Strukturen unter unterschiedlichen physikalischen Bedingungen in der Sonnenkorona wurde kritisch diskutiert. Dies wurde anhand einer besonderes schnellen, dynamischen Ereignisses, das in der grünen Koronalinie beobachtet wurde, illustriert.

Die Korona stellt das Bindeglied zwischen den unterschiedlichen Schichten der unteren Sonnenatmosphäre (Photosphäre, Chromosphäre und Übergangszone) und der ausgedehnten Heliosphäre dar, d.h. dem von Sonnenwindplasma erfüllten interplanetaren Raum. Aus diesem Grund ist das Studium der Rotation der Korona in Zusammenhang mit der bekannten differentiellen Rotation der Photosphäre von großer Bedeutung, um neue Erkentnisse über die Expansion der Korona, die Aufheizmechanismen und die Beschleunigung des Sonnenwinds zu gewinnen. In dieser Arbeit wurden deshalb die Rotationsmuster der grünen Korona anhand der neuen Koronagraphendaten untersucht. Wegen der enormen atmosphärischen Effekte bei den Beobachtungen mit einem erdgebundenen Korona-graph (MICA) und des Tag-Nacht-Zyklusses wurden Daten des nahezu baugleichen LASCOC1-Instruments auf dem Weltraumobservatorium SOHO verwendet. Die Ergebnisse deuten darauf hin, daß zwei unterschiedliche Rotationsmuster einander überlagert sind: Das eine entspricht "starrer" Rotation, d.h. gleiche Rotationsraten bei allen Breiten und Abständen und betrifft vor allem die langlebigen, mehrfach umlaufenden Strukturen. Dem ist überlagert das Muster differentieller, d.h. stark breitenabhängiger Rotation, für die eher kleinskaligen Strukturen. Letzteres Ergebnis wurde durch Analyse von Bildern der Sonnenscheibe bestätigt, die von SOHO-EIT in mehreren EUV-Spektralbereichen aufgenommen wurden. 



\section{Chapter 1}

\section{Introduction}

Many things in nature give us ideas about how to exploit natural phenomena. For example, Man wanted to fly since the first bird was seen to fly. In ancient times, eclipses revealed extraordinary features not understood at all. Intrigued by that, astronomers began to build instruments to simulate eclipses in analogy to the engineers who created planes to emulate the flight of birds. Generating an idea is not a formidable task, the formidable task is the creation of the necessary technology to pursue the idea and finally prove it.

The only way to observe the "tongues of fire" coming out of the Sun was, until the 1930's, when the Moon, Sun and Earth are in the peculiar position to produce a total eclipse. If the distance Earth-Moon were not such to produce an angular diameter of the Moon slightly larger than that of the Sun, we would have never seen or even suspected the Sun's extended atmosphere, missing precious information. How many things are we still missing simply because we do not have at hand the right tools to inquire nature? It's not the purpose of this work to discuss such philosophical thoughts in too much detail but I personally consider it important to keep an open mind on such basic issues.

One of the most beautiful displays on the sky we get from nature appears during total eclipses (see, e.g., Figure 1.1), when the Sun's extended atmosphere becomes visible against a darkened sky. Many references to observations of such events can be found in the literature. The few minutes of totality available approximately once a year during a solar eclipse stimulates us to learn more about the extended and expanding atmosphere of the Sun. However, it was not until 1842, when a total solar eclipse crossed southern Europe, that the solar corona became object of systematic scientific research. At that time, scientists were skeptical about the solar origin of the features seen in eclipses. The application of new photographic methods and of spectroscopy in 1860 and thereafter gave new insight and transformed eclipse observations into vital experiments about the nature of the Sun. In 1869, spectroscopic observations carried out by W. Harkness and by C. A. Young during a solar eclipse at the United States, led to the discovery of the green coronal line. Two years later, Janssen found Fraunhofer lines in the coronal spectrum, this fact proving the solar origin of the coronal light and showing that it is at least in part due to scattered sunlight. Thanks to the invention in 1930 by B. Lyot of a device to produce artificial eclipses, the so-called "coronagraph", the natural and fascinating spectacle became more readily accessible. This gave us the possibility to observe the tenuous and faint solar atmosphere, without having to wait for the rare occasions when a solar eclipse occurs. This instrument (described in Lyot, 1931) contained special features to reduce the instrumentally scattered radiation and allowed routine coronal observations from mountaintop observatories with clear skies.

By the early 1930's, when the observation of the solar corona was not any longer restricted to times of solar eclipses, the physical interpretation of these observations was still in a state of complete confusion; with the thermodynamic and spectroscopic premises of that time, the existence of the corona could not be explained. First, the corona was seen to extend 100 times further from the solar surface than expected. Second, the coronal spectrum 


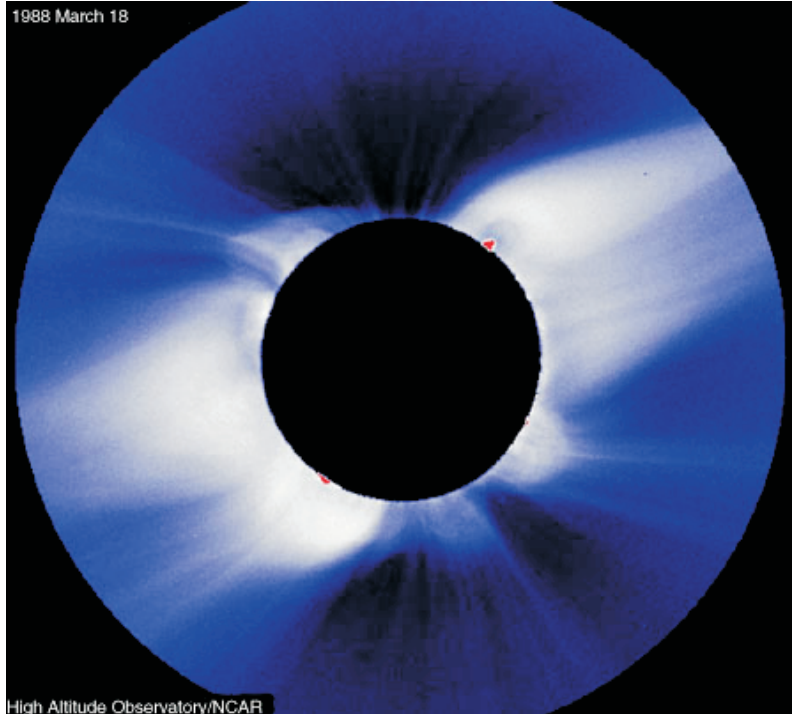

Figure 1.1: Total solar eclipse image of March 1988 taken from a site located in Philippines by an expedition of the High Altitude Observatory of Boulder, Colorado.

was observed to consist of a continuum with an assembly of emission lines. The inability to identify these emission lines had been the most outstanding problem in astrophysics for 50 years. The big breakthrough occurred when Grotrian (1939) showed that two of the observed coronal lines correspond to forbidden transitions ${ }^{1}$ of the $\mathrm{Fe}^{9+}$ and $\mathrm{Fe}^{10+}$ ions. Grotrian based his calculations on measurements made by the swedish spectroscopist B. Edlén (1936, 1937). Edlén proceeded to identify further 17 coronal lines, all of them emitted by highly ionized atoms (see, e.g., Chapter 2 of Shklovskii, 1965 for a detailed discussion). Hence, Grotrian and Edlén showed that the strange coronal lines are emitted by elements such as iron, calcium and nickel in very high stages of ionization. The presence of these ions unequivocally indicated temperatures in the corona of at least one million degrees, necessary for the formation of these ions. Such high temperatures could also explain the great height of the corona ${ }^{2}$ above the solar surface.

The existence of the hot corona explained a lot of observational facts but rose other fundamental questions such as: where does the coronal material come from and how is it heated? In 1957, Sydney Chapman proposed that the corona should extend out into interplanetary space (out to $1 \mathrm{AU}$ and probably much beyond) rather than being confined to a thin layer above the solar surface, like in planetary atmospheres (Chapman, 1957). In his model (corona in static equilibrium), the pressure at infinity remains much higher than that of the interstellar background unless one assumes an unreasonably low temperature for the inner corona. In other words, the corona cannot be

\footnotetext{
${ }^{1}$ In complex ions, there are an enormous number of possible transitions. Not all of these possible transitions are observed. This is because some transitions are more likely than others. The term permitted lines is used for those transitions whose transition probability is high, i.e., $A \approx 10^{8} \mathrm{sec}^{-1}$. On the other hand, forbidden lines have low transition probabilities, because they cannot radiate in a dipole transition as is the case for permitted lines. Forbidden transitions often occur through magnetic dipole $\left(A \approx 1 \mathrm{sec}^{-1}\right)$ or electric quadrupole $\left(A \approx 10^{-4} \sec ^{-1}\right)$ transitions (see, e.g, Chapter 5 of Zirin, 1988). They are principally of importance in tenuous gases, such as the corona or the interstellar medium (because of the extremely low densities, the lifetime of forbidden transitions is shorter than the mean time between collisions).

${ }^{2}$ For an isothermal atmosphere, the "density scale height", i.e. the distance over which a $1 / e$ drop in density occurs, is $k T / m g$, where $T$ is the temperature of the atmosphere and $m$ is the mass of a typical atom or particle of the gas. It is clear that for an extended atmosphere must be either $T$ large or $m$ small.
} 


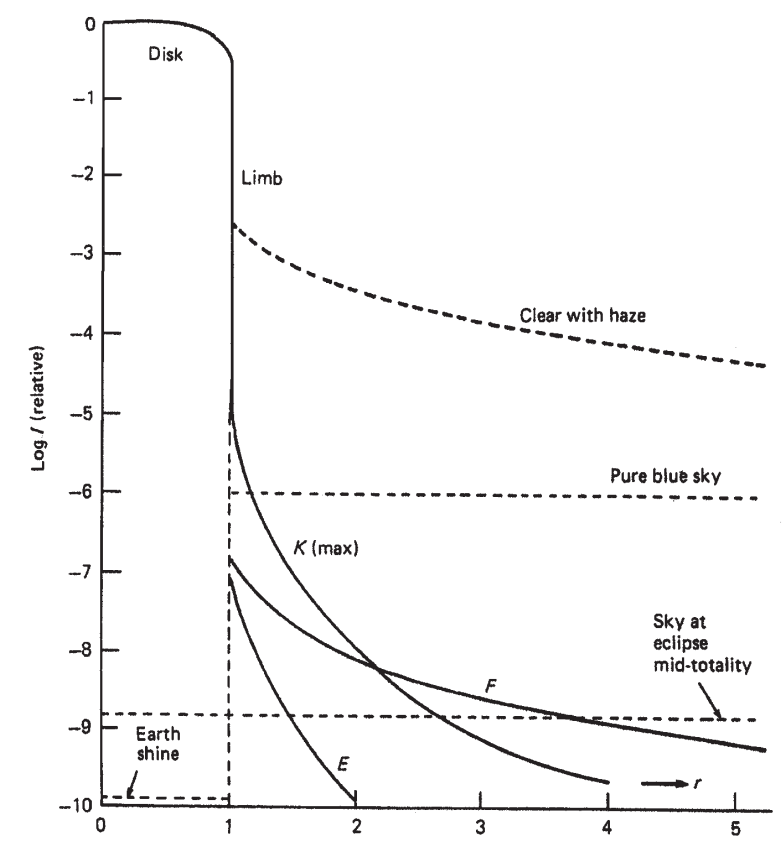

Figure 1.2: Relative intensity of the coronal light components and the sky as function of solar distance (in solar radii). The relative intensity is normalized to the intensity at the center of the solar disk. E: emission line corona; F: Fraunhofer corona; K: continuum (“white light”) corona. After van der Hulst, 1953.

contained. One year later, Parker (1958) showed that the physical inconsistency in the Chapman model implies that the corona is not merely extended, but must necessarily be expanding into interplanetary space. That is what he called "solar wind", a term which became of common use among the solar physicists.

Theoretical developments as the aforementioned were later on observationally confirmed. That marked the direction in which solar research would go in the future.

The first and natural way of studying the Sun's atmosphere has been with coronagraphs from Earth. Groundbased observations in visible light complement eclipse observations, providing basic steps in our long way to the final understanding. Unfortunately, observing the faint solar corona is strongly limited by the day-time brightness and variability of the Earth's atmosphere, as well as by the residual scattered light inside any instrument. For illustration purposes, the radial variation of the relative intensity of the sky under several atmospheric conditions in comparison to the different components which conform the coronal emission is shown in Figure 1.2. Knowledge of the characteristics of these components allows the implementation of instruments and reduction techniques to isolate their emissions from the relative high intensity of the sky, and reveal in this way the mechanisms responsible for such emissions. The different components are labelled the K- ("Kontinuerlich”), F- (Fraunhofer), and E(Emission) corona. These three components are now known to be due to very different mechanisms. The K-corona displays a continuum emission spectrum. Its light is found to be strongly polarized parallel to the solar limb. It arises from photospheric white light scattered on free electrons in the partly ionized corona (Thomson scattering). The absence of Fraunhofer absorption lines in the K-corona spectrum is due to the high electron temperature in the corona, causing Doppler smear-out. On the other hand, the F-corona shows the same spectrum found for the photosphere, characterized by the presence of dark absorption lines (Fraunhofer lines). It arises from scattering of the photospheric light by small dust particles in the ecliptic plane, and is more accurately thought of as the 
inner "zodiacal light". It shows a very low degree of polarization. The E-corona represents the only component that arises from the emission of light by the coronal gas, i.e., the emission of highly ionized ions. Recently, a fourth component was identified, the T- (Thermal) corona, which is caused by thermal (largely infrared) emission of interplanetary dust particles.

Detailed knowledge of the observational characteristics of the different corona components leads to the understanding of the physical mechanisms responsible of the emission, permitting their separation. The use of polarizers, for example, allows the isolation of the K-corona. On the other hand, the use of narrow band filters centered on the desired wavelength increases the contrast of the emission lines relative to the background emission allowing in this way their detection. Furthermore, background subtraction techniques, as will be shown in detail in Chapter 3 , make possible the almost complete isolation of such lines. Nevertheless, the high variability of the sky still puts constraints to ground-based observations.

The advent of space technology made possible to overcome the large limitations imposed by the Earth's atmosphere. Observations from space are not affected by turbulent motions in the atmosphere nor subjected to changes in sky brightness. Furthermore, by operating outside the atmosphere, the observations could be extended to other spectral ranges not visible from Earth due to the absorption suffered in the Earth's atmosphere. The X-EUV spectral range is a typical example where, in addition, the corona can be observed without any perturbation from the solar disk. An excellent review by Koutchmy (1988) accounts for the main aspects of coronal observations from space and the historical development of white-light, externally-occulted coronagraphs ${ }^{3}$.

Until recently, there was an observational gap between close-to-limb early coronagraph (both ground and space based) observations and what we know from eclipses. The externally-occulted coronagraphs used so far in space missions suffered from vignetting at the inner edge of the occulter and did not allow useful observations inside about $2 \mathrm{R}_{\odot}$ (see Chapter 2). Newkirk and Bohlin (1963) pointed out in the 1960's that the residual instrumental straylight can be reduced considerably by the use of mirror optics instead of lenses. Small, externally occulted rocket- and ballon-borne coronagraphs have successfully made use of reflective optics (see, e.g., Kohl et al., 1978). Therefore, the use of a "coronagraphic quality mirror" as a primary objective would allow to go back to the original Lyot concept of internally-occulted coronagraph. Recently, extremely low scatter mirror development has allowed the use of such mirrors as objectives for internally-occulted coronagraphs (see, e.g., Smartt et al., 1990). LASCO-C1 aboard SOHO (Brueckner et al., 1995) and a prior prototype (Epple \& Schwenn, 1994) were the first internally-occulted coronagraphs designed with reflective optics. An excellent work about the design of a mirror coronagraph with a detailed study of the instrumental straylight problem can be found in Epple (1997).

Following this line, a third mirror coronagraph was built as a by-product of the instruments previously mentioned. The so-called MICA (Mirror Coronagraph for Argentina) is almost identical in design to the LASCO-C1 instrument. Therefore, it incorporates all the advantages of internally occulting and mirror design such as compactness, low level of instrumental scattered light and optical achromacy, to name the most important ones. The in-

\footnotetext{
${ }^{3}$ The so-called externally occulted coronagraphs use a design introduced by Evans (1948) in the Lyot optical scheme to shield the objective lens from direct photospheric radiation and thus reduce the instrumental straylight level. Briefly, a circular occulting disk is located at a substantial distance in front of the entrance aperture, so that the Sun is artificially eclipsed and no direct sunlight falls into the instrument. In Chapter 2 this topic is addressed in deeper detail.
} 
strument, built by the Max-Planck-Institut für Aeronomie, is part of a bilateral science program between Germany and Argentina. The institutions involved in this program are, for Germany, the Max-Planck-Institut für Aeronomie (MPAe) at Lindau, and the Max-Planck-Institut für Extraterrestrische Physik (MPE) at Garching; and on the Argentinean side, the Instituto de Astronomía y Física del Espacio (IAFE), Buenos Aires, as part of the Argentinean National Research Council (Consejo Nacional de Investigaciones Científicas y Técnicas, CONICET) and the Observatorio Astronómico Félix Aguilar (OAFA), San Juan, as part of the San Juan University. The bilateral science program is aimed at establishing a wide cooperation in the area of Solar Physics Research between the institutions involved. It includes joint elaboration and development of technological and scientific research programs, professors, researchers and students exchange, updating and training of personnel, and participation in joint publications.

The MICA telescope is located at the Prof. Ulrico Cesco High Altitude Station of OAFA at El Leoncito $\left(31.8^{\circ} \mathrm{S}\right.$, $\left.69.3^{\circ} \mathrm{W}\right)$, San Juan, Argentina at $2400 \mathrm{~m}$ altitude, in the western part of the mountain chain "El Tontal" in the Argentinean Precordillera, 50 km eastwards from the "Cordillera de los Andes" (Figure 1.3). Due to the "shadow" effect of the high Andes mountains, the air is usually dry and clean, and similarly good weather conditions prevail throughout the year. The strategic location in the southern hemisphere allows a complementary summer-condition data with respect to the other solar observatories, all located in the northern hemisphere.

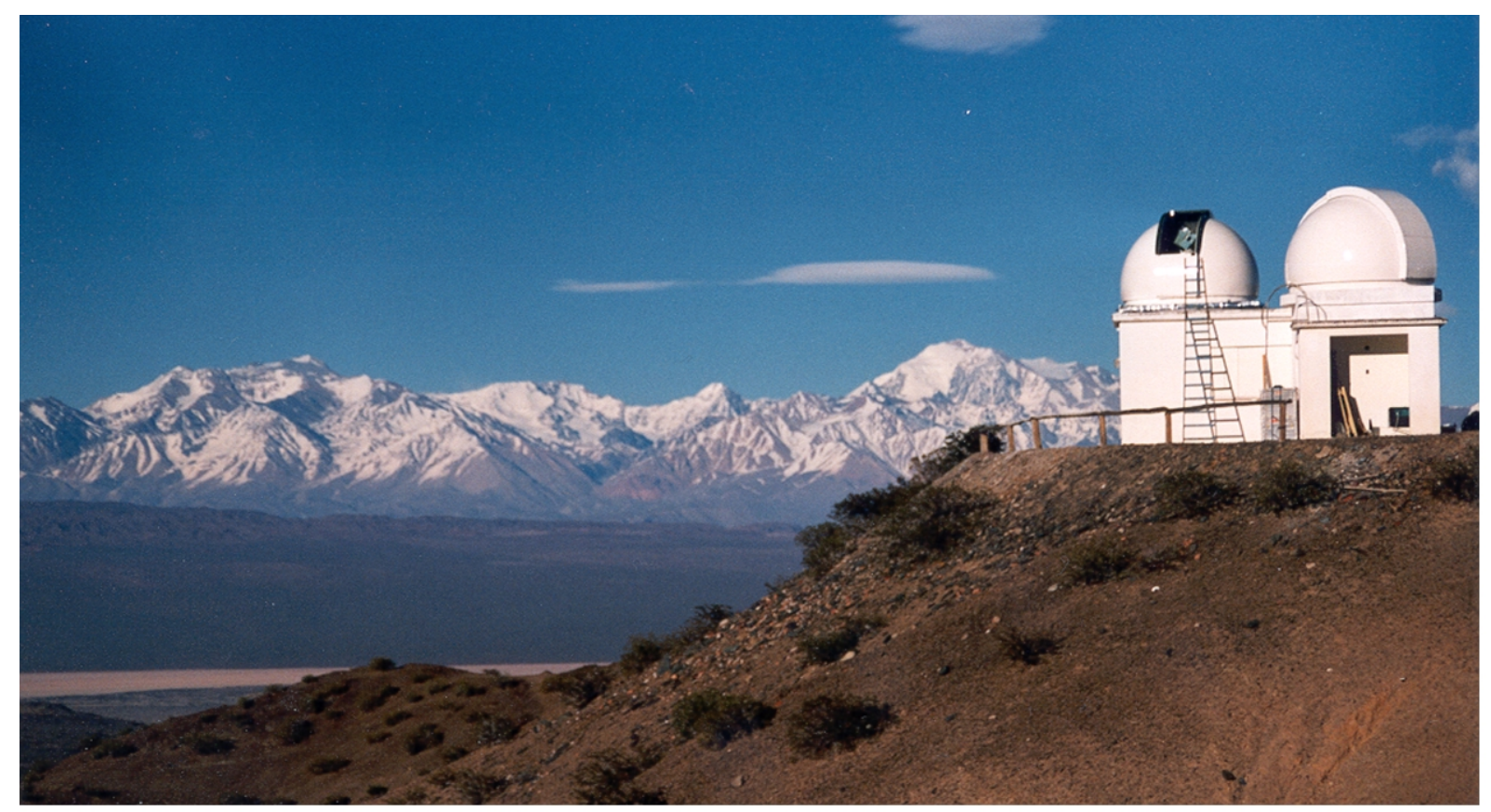

Figure 1.3: Panoramic view of El Leoncito, with the Andes chain in background (6000 $\mathrm{m}$ high in average). On the right, the MICA and HASTA domes. 


\subsection{Outline of the Thesis}

Observations are the prime source to know what is actually happening in the object under study, i.e., the Sun in the present case. They provide constraints to proposed models and ultimately are the means by which the different mechanism proposed in the models are tested. Therefore, the thesis deals with the interpretation and analysis of coronal observations on various time scales. Images from the ground-based telescope MICA are used for the analysis on short time scales. Since MICA has been recently installed (August, 1997), several steps had to be first carried out, such as: i) the design and development of a control software to integrate the different components of the MICA telescope, which allows its automatic and stand-alone operation, and ii) the calibration of the observations obtained by MICA.

The thesis consists of six chapters, the brief review presented here being the first one. In Chapter 2, the MICA instrument is introduced with a brief but exhaustive description and background of its optical components. Further, the noise characteristics of the MICA camera are discussed. One of the main features of the MICA system is its stand-alone operation. Several auxiliary devices help the instrument in such a task, all of them commanded and controlled by a control software specially designed. After briefly describing such devices, an overall review of the software developed is presented in the last part of Chapter 2.

The mathematical foundation of the several steps necessary to reveal the faint coronal features is elaborated in Chapter 3. Special attention is paid to the calibration of the observations relative to the intensity at the center of the solar disk. In order to analyze the reliability of our calibration procedure, the influence of the short-term variability of the extinction coefficient in the site is analyzed. Comparison with calibrated data from the Sacramento Peak Observatory is performed. Finally, a summary of the aspects to have in mind when interpreting coronal observations is given.

The study of dynamical events in the inner corona is crucial for a better understanding of the processes involved in the triggering and release of coronal mass ejections. The first step to this effect is the correct identification of the observed coronal features. In Chapter 4, a discussion on the interpretation of the observed structures and reliability of the calibration procedure under different physical conditions in the corona is presented. The discussion is supported by several examples. In particular, a fast dynamical event observed in the green emission line is carefully analyzed. Combined observations with the other instrument in El Leoncito, i.e., HASTA (Appendix C), are used to help in the understanding of the mechanisms at work at the triggering and during the evolution of these events.

The inner corona establishes the necessary connection between the mechanisms operating at the lower layers of the Sun's atmosphere, i.e., photosphere, chromosphere, and transition region, and the outer heliosphere. For that reason, the study and analysis of the rotation rate of the corona as measured in the green line emission is of fundamental importance to provide new constraints to the existing models of the coronal expansion and its relation to the solar wind and heating mechanisms. Chapter 5 concentrates on the analysis of the recurrence of the green line pattern. Since atmospheric effects enormously affect ground-based coronagraph observations, as well as the day-night cycle and weather conditions avoid having a continuous data set, data from LASCO-C1 on board SOHO have been used for that study. For comparison, tracking of stationary coronal features at different wavelengths is 
also used to estimate the rotation rate at different coronal heights. Images from the EIT instrument on board SOHO have been used in this case.

Finally, the last chapter summarizes the conclusions drawn from the present study as well as places in perspective the MICA system and its contribution to the scientific community. 



\section{Chapter 2}

\section{The Telescope and the Auxiliary Systems}

In the present chapter, I first mention the basic design drivers for the MICA telescope as an internally occulted mirror coronagraph. Then, I review the instrument layout and describe the different components, in order to explain the constraints we have to deal with. Finally, the control of the system and the steps performed to achieve the automatic control of the telescope are described.

\subsection{Introduction}

In the past, the observation of the solar corona was restricted to times of solar eclipses. Only since the invention of the coronagraph by B. Lyot in 1930, the extended and tenuous gas around the Sun can be observed at high altitude observatories under good observing conditions. Unfortunately, the observations made in ground-based observatories are fundamentally limited by the sky brightness, even at high altitudes. The advent of space technology allowed to overcome the limitations imposed by the Earth's atmosphere, giving rise to space-based coronagraphy. Even so, any straylight produced inside the instrument masks many interesting parts of the corona.

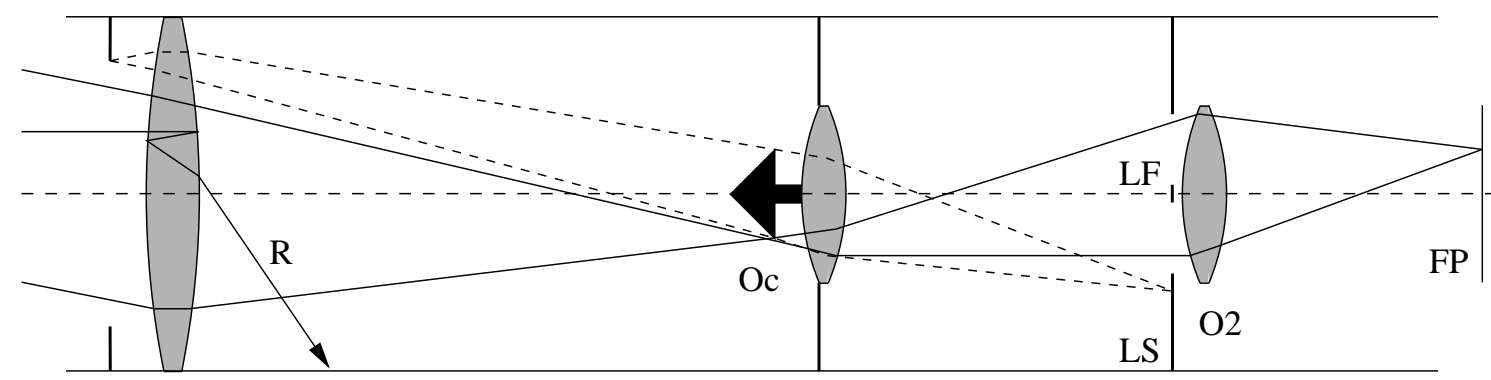

A1 O1

FL

Figure 2.1: Optical scheme of a conventional Lyot refractive internally occulted coronagraph. A0: entrance aperture, O1: objective lens, Oc: internal occulter, FL: field lens, LS: Lyot stop, LF: Lyot spot, O2: transfer lens, FP: focal plane. The solid lines show the rays coming from a point in the inner corona. The dashed line indicates the path of the diffracted light at the edges of the entrance aperture. Internal reflections in the objective lens are denoted with the letter $R$.

The optical layout of a conventional Lyot coronagraph as described in Evans (1953) is given in Figure 2.1. Lyot observed that each optical element or edge illuminated by solar radiation gave a contribution to the straylight level of the coronagraph. The first step in discarding the solar radiation was placing a circular disk or solar occulter $(\mathrm{Oc})$ at the focus of the objective lens (O1) to intercept and discard the solar disk image. The principal sources of stray disk radiation after the occulter were scattered radiation from i) the objective lens (O1), ii) any edges directly 
illuminated by solar radiation, in particular those of the entrance aperture (A0), and iii) internal reflections in the objective (R). A secondary solar image produced by multiple reflections in the lens appeared at the position of the so-called Lyot spot (LF). Behind the occulter (Oc), a field lens (FL) images the coronagraph aperture edges (A0) onto a pupil known as the Lyot stop (LS). This stop eliminated the diffracted solar radiation arising at the edges of the entrance aperture. The Lyot spot was also eliminated by a mask placed behind the Lyot stop. With the removal of the aperture edges and the Lyot spot, the residual straylight originated at the objective lens.

A design introduced by Evans (1948) in the Lyot (1939) optical scheme allowed to reduce the instrumentally scattered solar light by shielding the objective lens from direct photospheric radiation. The so-called "external occultation" design uses a device external to the main objective (at a substantial distance in front of the entrance aperture) to occult the solar disk and produce a shadow, yielding in this way an artificial solar eclipse.

In the externally occulted version of the conventional Lyot-type coronagraphs, two main types of residual straylight are still unavoidable: i) diffracted sunlight from the edge of the external occulting disk (the largest one), and ii) residual scattered light in the objective lens itself. On the other hand, monochromatic and chromatic aberration $^{1}$ at the position of the internal occulter is a serious drawback of single-lens objectives. Because of chromatic aberration, the optimum size and position along the optical axis of the image of the external occulting disk is wavelength dependent. This precludes simultaneous observation at different wavelengths. Another limitation of externally occulted coronagraphs is that the spatial resolution is strongly degraded at the inner edge of the field of view due to the vignetting characteristics of this kind of telescopes ${ }^{2}$. The degradation is roughly proportional to the vignetting effect in the radial direction, so the effective resolution of an externally occulted coronagraph is always a function of the radial distance. For the inner field of view, the diffraction limit ${ }^{3}$ is set by the distance and size of the external occulter, while for the outer field of view, it is set by the effective aperture of the objective lens. Further, dimensional constraints impede to build sufficiently long externally occulted coronagraphs to observe the innermost corona with high spatial resolution. Therefore, useful observations inside about $1.5 \mathrm{R} \odot$ are not possible with externally occulted coronagraphs.

In order to preserve the full resolution over the entire field of view and achieve images of the solar corona as close to the solar limb as possible, the internally occulted version of the Lyot coronagraph remains a valid option. Thus, the problems inherent to the external occulted disk as variable spatial resolution and straylight due to diffraction at the inner edges of the occulter can be overcome. However, a remaining constraint, besides that of the instrumental straylight in internally-occulted Lyot-type coronagraphs, is that they have an obvious aperture limitation due to the problem of producing large diameter slabs of glass of requisite quality for the primary objective ${ }^{4}$.

\footnotetext{
${ }^{1}$ Chromatic aberration occurs due to the variation of refractive index with wavelength for a lens material. This wavelength dependence results in slightly different focal lengths for different wavelengths of light. Therefore, the lens produces a coloured blurring rather than a true color, sharply focussed image.

${ }^{2}$ The vignetting is mainly due to the presence of an external occulting disk at a relative short distance of the objective lens, which acts as a limiter to the effective aperture of the telescope.

${ }^{3}$ The diffraction limit is the minimum angular separation that two different sources must have in order to be resolved by the telescope.

${ }^{4}$ Large diameter refractive lenses are impractical to build. First, they have a number of aberrations, in particular chromatic aberrations. A second problem with refractive telescopes is that the bending necessary for focussing depends strictly on a curved shape, hence there is no
} 
The instrumental straylight can be considerably reduced by the use of reflective optics instead of lenses as already pointed out by Newkirk \& Bohlin (1963). The use of mirror objectives eliminates, in addition, the chromatic aberration difficulties at the occulter inherent to lens objectives. Unlike lenses, mirrors have no problems with bulk scatter or multiple internal reflections. Further, with mirrors objectives large apertures are feasible. Note also that the likelihood of dust contamination is reduced as compared with having a lens objective at the entrance aperture since the mirror objective is located towards the rear of the instrument, where it is well protected against contamination from outside (compare, e.g., figures 2.1 with 2.2). Mirror objectives for coronagraphs have already been proposed (e.g., Newkirk and Bohlin, 1963) and successfully applied to small, externally occulted rocket and balloon borne coronagraphs (Bonnet, 1966; Kohl et al., 1978; Smartt, 1979). Presently, mirrors with straylight level down to a few $10^{-8} \mathrm{~B}_{\odot}$ are available.

The development of reflecting coronagraphs has now reached a level where it is clear that these new-technology instruments can fulfill many of the observational needs of contemporary coronal physics. Apart from the advantages of achromaticity, large wavelength coverage, and low instrumental polarization, they can have relatively large apertures with resultant gains in angular resolution and sensitivity.

The coronagraph covering the innermost range of the three SOHO visible light coronagraphs, i.e., LASCOC1 (Brueckner et al., 1995) and a prior prototype (PICO ${ }^{5}$, Epple \& Schwenn, 1994) were the first internally occulted coronagraphs designed with reflective optics. Following this line, the MICA telescope incorporates all the advantages of internally occulting and mirror design such as compactness, low level of instrumental scattered light, and optical achromacy, to name the most important ones.

\subsection{The optical layout of the MICA telescope}

The MICA telescope (Stenborg et al., 1999a,b) is almost identical in design to the LASCO-C1 instrument on board SOHO (Brueckner et al., 1995). The coronagraph is enclosed in a rectangular box made from one piece of Aluminium $(1353 \mathrm{~mm} \times 340 \mathrm{~mm} \times 173 \mathrm{~mm})$, this box being strong enough to serve as optical bench for the optical elements. The optical layout of the instrument is shown in Figure 2.2.

- The entrance aperture $\mathrm{A}_{0}$ (47 mm diameter) allows the full Sun, coronal light, and scattered sunlight in the terrestrial atmosphere, to illuminate the objective off-axis parabolic mirror $\mathrm{M}_{1}$ (75 $\mathrm{cm}$ focal length). Moreover, the diffraction pattern produced in $\mathrm{A}_{0}$ also contributes to the illumination of $\mathrm{M}_{1}$. Inhomogeneities, dust particles, and surface imperfections of $\mathrm{M}_{1}$ contribute mostly to the instrumental straylight ${ }^{6}$. The aperture of

\footnotetext{
alternative to having a thick lens. Glass is actually a slow-moving liquid rather than a solid. It bends and flows under its own weight. A perfectly shaped lens will soon flow into an undesirable shape unless it is supported. The support of course cannot help, but interfere with light gathering. ${ }^{5}$ Pic Du Midi Coronagraph.

${ }^{6}$ The dominant source of straylight in an internally-occulted Lyot-type coronagraph is near specular scattered solar radiation in the first optical element (Lyot, 1939; Newkirk \& Bohlin, 1963.)
} 


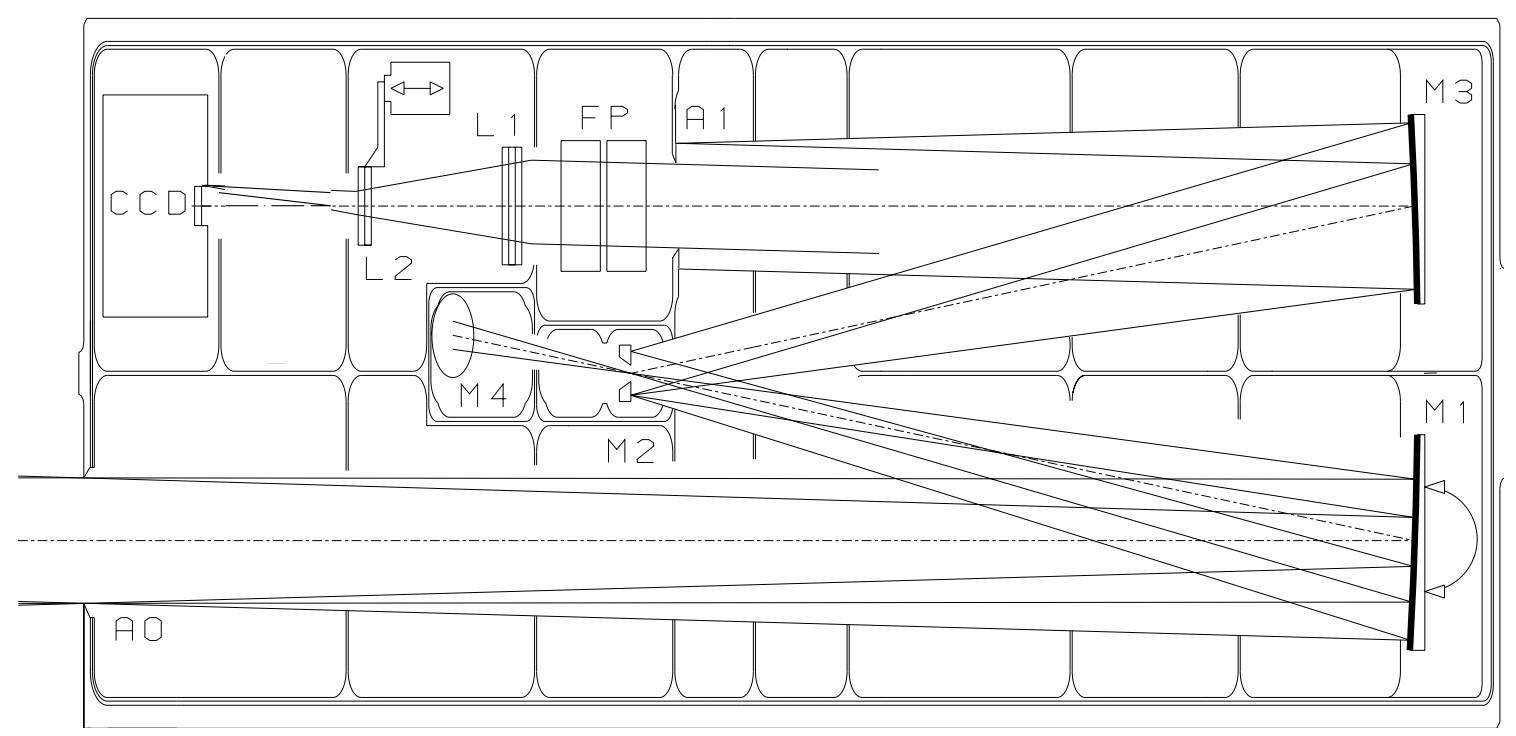

Figure 2.2: Optical layout of the internally-occulted mirror coronagraph MICA. A0: entrance aperture, M1: objective mirror, M2: field mirror, M4: diagonal mirror, M3: collimating mirror, A1: Lyot stop, F: Filter box, L1-L2: telelens system, CCD: camera. For details see text.

$M_{1}$ is sufficiently larger than that of $A_{0}$, so that $A_{0}$ is the only source of diffracted light.

- A real image of the Sun and the corona is formed at the position of the convex spherical mirror $\mathrm{M}_{2}(242 \mathrm{~cm}$ radius of curvature) which thus acts as a field mirror. Furthermore, $\mathbf{M}_{2}$ acts as an "internal" occulter for the light from the bright solar disk simply because there is a hole in $\mathrm{M}_{2}$ corresponding to $1.05 \mathrm{R}_{\odot}$. The solar disk image passes through it and is eliminated from the system using the diagonal mirror $\mathbf{M}_{4}$.

- The field mirror $\mathrm{M}_{2}$ reflects the remaining light (outside a radius of $1.05 \mathrm{R}_{\odot}$ ) on to an off-axis parabolic collimating mirror $\mathbf{M}_{3} . \mathrm{M}_{3}$ is identical to $\mathrm{M}_{1}$, with the same axis and focal point. In fact, $\mathbf{M}_{1}$ and $\mathbf{M}_{3}$ can be thought of as one single mirror. They are placed symmetrically, forming segments of a single parabolic mirror. This fully symmetric arrangement accomplishes the cancellation of coma in the system.

- Since the curvature of $M_{2}$ is chosen such that an image of $A_{0}$ is formed in the plane of the stop $A_{1}$, the scattered light emerging from the fully Sun-illuminated edge of $\mathrm{A}_{0}$ is intercepted at $\mathrm{A}_{1}$ which thus acts as a Lyot stop. In addition, there is an array of baffles installed in order to minimize the amount of scattered light.

- The collimated beam leaving $A_{1}$ is sent through a selected interference filter into an achromatic telelens system, and is focussed on a CCD camera (Section 2.4). The layout is such that a circular field of view with a radius of about $2 R_{\odot}$ forms an inscribed circle on the CCD.

\subsection{The filters}

The basic idea behind narrow-band imaging is that for each extended object two images have to be taken: 
- One image through a narrow-band interference filter which isolates the emission line of interest (including the underlying continuum and instrumental straylight, and in the ground-based case, the scattered light from the terrestrial atmosphere),

- The other one through another interference filter isolating an adjacent region of the spectrum containing only continuum light (and, as aforementioned, instrumental straylight and the sky).

In the following discussion I will refer to the former as the on-line image, and the latter as the off-line one. A map of the emission-line flux is obtained by subtracting a scaled version of the off-line image from the on-line one, to remove the contribution in the on-line image due to the underlying continuum, instrumental straylight and sky. The linearity and dimensional stability of the present imaging devices, i.e., charge coupled devices, makes this difference technique straightforward. Effective application of this technique requires a brief review of the properties of interference filters and the choice of filters to use.

\subsubsection{Theoretical background}

Strictly speaking, an interference filter is a solid-body Fabry-Perot interferometer. The simplest one, the so-called one-cavity filter, consists of a dielectric layer sandwiched between two quarter-wave reflector stacks, usually made of alternating thin layers of high- and low-index materials deposited on a glass substrate. The properties of the filter are controlled by the number and thickness of the layers and the use of multiple cavities as well. The bandpass of the filter is well described by 5 parameters: i) the central wavelength $\lambda_{c}$, ii) the peak transmission $T_{\text {peak }}$, and three width parameters: the full width at 50\%, $90 \%$ and $1 \%$ (i.e., $W_{50}, W_{90}$, and $W_{1}$ respectively). The first one is usually referred to as Full Width at Half Maximum (FWHM). In general, to a good approximation, $W_{90}=0.6 \cdot W_{50}$ and $W_{1}=2 \cdot W_{50}$.

Let us consider an idealized filter observing an idealized spectrum in order to establish guidelines for determining the optimum filter passband for a particular problem. The total light flux passing through a filter centered at $\lambda_{c}$ is proportional to the convolution between the flux entering the filter and the transmission profile of the filter. Therefore, we assume that the line emission source (immersed in a continuous background) to be detected has a rectangular profile of spectral width $\omega$ corresponding to a characteristic resolution $\Re_{c}=\lambda / \omega$. On the other hand, the passband profile of the filter is likewise rectangular of width $W$ corresponding to a filter resolution of $\Re=\lambda / W$. In this case, it can be easily demonstrated (Roesler, 1974) that in the photon limited case (i.e., when the dark response of the detector system can be considered negligible), the functional dependence of the resolution/contrast curve $^{7}$ is characterized by $\left(\mathfrak{R} / \mathfrak{R}_{c}\right)^{0}$ if $\mathfrak{R} / \mathfrak{R}_{c}<1$ and $\left(\mathfrak{R} / \mathfrak{R}_{c}\right)^{-1}$ if $\mathfrak{R} / \mathfrak{R}_{c}>1$ (note that $\mathfrak{R} / \mathfrak{R}_{c}=\omega / W$ ). In other

\footnotetext{
${ }^{7}$ The line contrast may be defined as the ratio of the line contribution to the noise, i.e.,

$$
E=\frac{\left|F_{2}-F_{1}\right|}{\left(F_{2}+D\right)^{1 / 2}}
$$

where $F_{1}$ is the continuum contribution, $F_{2}$ is the total contribution (i.e., line plus continuum), and $D$ the dark response of the detector system. The noise is assumed to be photon noise (Section 2.4.1) and given by $\left(F_{2}+D\right)^{1 / 2}$. The dependence of $E$ on the ratio of the instrumental resolution to the source characteristic resolution for the idealized source and instrument $(\mathfrak{R} / \mathfrak{R})$ is here referred as the resolution/contrast curve.
} 
words, by choosing a filter with wider $W_{50}$ (i.e., the FWHM), the dispersion is increased (i.e., increasing of the spectral coverage). In this case, more continuum photons are transmitted while the number of line photons remains the same. Thus, the relative amount of continuum photons is increased and the line contrast decreases as $1 / W_{50}$. Eventually, the contrast gets so low that the line is lost in the noise. However, if the spectral resolution of the filter is increased (i.e., by decreasing $W_{50}$ ), after reaching the point where the filter half-width is equal to that of the emission line, there is no further gain with increasing resolution, and the resolution/contrast curve flattens out.

On the other hand, the lines of interest should fall within the range defined by $W_{90}$, as with most 2 - or 3-cavity interference filters the bandpass is more or less flat-topped in this range. Finally, the filter's $1 \%$ width $\left(W_{1}\right)$ should reject adjacent emission lines from the bandpass.

\section{Interference filters systematics}

There exist a number of systematic properties affecting the true bandpass of interference filters. Relaxation of either of these conditions can substantially alter the bandpass. First and foremost is that the bandpass of the filter is always specified in the laboratory for parallel light passing through the filter at normal incidence. Tilting of the filter with respect to the beam by an angle $\theta$, will cause the bandpass to shift to the blue from its lab bandpass center $\lambda_{0}$, according to the formula:

$$
\lambda_{\theta}=\lambda_{0} *\left(1-\sin ^{2} \theta / N^{2}\right)^{1 / 2}
$$

where $N$ is the index of refraction of the filter. Note that this systematic may be used to fine-tune a filter bandpass by tilting the filter in the telescope beam to best match the emission-line wavelength of interest. At large angles (typically for $\theta>30$ degrees) polarization effects begin to seriously distort the bandpass.

Since the bandpass depends on the angle of incidence of the rays entering the filter, i.e., $\theta$, it is thus obvious that the bandpass is affected if the filter is used in a converging or parallel beam. One way is using the filters in the so-called telecentric mode, in which the telescope objective is collimated through the filter, the filter being placed near the image plane. In this case, all the image points are formed by ray cones normally incident on the filter, containing all the possible directions (given by $\theta$ ) allowed by the optics. The FWHM of the filter becomes thus larger because the raybundle corresponding to each image point contains a range of $\theta$ values. To avoid broadening of the bandpass, filters are therefore mostly used in the optical configuration known as classical mounting, where the image formed by the telescope is collimated and the filter is placed near the image of the entrance pupil. In this case, each image point is formed by a beam of rays propagating through the filter at the same angle with respect to the optical axis. The drawback is that the final image plane is spectrally inhomogeneous, in the sense that the transmitted wavelength is not the same on each point, but depends on its distance from the optical axis (i.e., each off-axis image point subtends a different $\theta$ angle, resulting in a blueshift of $\lambda_{0}$ according to (2.1)). In both cases, the small scale flatness errors of the surface also widen the FWHM of the bandpass, this effect being larger in the classical mounting. Therefore, filter flatness should be "imaging quality".

An important but often overlooked problem is environmental sensitivity, in particular temperature sensitivity. Thermal effects in interference filters affect primarily the thickness of the dielectric layers. Therefore, laboratory 
filter bandpasses are usually measured at $20 \mathrm{C}$. The use of the filters at other temperatures results in a red- or blueshift of the bandpass. According to the technical documents of most thin-film filter manufacturers, the approximate bandpass shift is $0.18 \AA / \mathrm{C}$, with the bandpass red-shifting for $T$ warmer and blue-shifting if colder.

Finally and quite important is that all interference filters degrade with time as water vapour diffuses into the filters and breaks down the coatings. The usual effect of aging is for the filter bandpasses to slowly blueshift with age.

\subsubsection{The filters in MICA}

The spectral range of the instrument is selected by using a set of narrow-band interference filters $(0.15 \mathrm{~nm})$, corresponding to the emissions of the well-known Fe XIV green and Fe X red coronal emission lines at $530.3 \mathrm{~nm}$, and $637.4 \mathrm{~nm}$, respectively (Table 2.1). The continuum emission is taken with a relative broad-band interference filter at a wavelength sufficiently far from line center, i.e., at $526.0 \mathrm{~nm}$ for the green line, to avoid contamination by emission in the line itself. For the red line, a similar filter to that for the green line, with central passband at $634.0 \mathrm{~nm}$, is used. Since the off-band images are to be used as a model of the continuum in the on-line images, the auxiliary filters had to be chosen with a relatively broad passband (i.e., $\sim 1 \mathrm{~nm}$ ), to be sure that no major emission lines could contribute to the measured continuum flux (see the discussion in Section 2.3.1).

\begin{tabular}{ccccccc}
\hline \hline$\lambda$ & $\begin{array}{c}\text { FWHM } \\
(\mathrm{nm})\end{array}$ & $\begin{array}{c}\text { Max. } \\
(\mathrm{nm})\end{array}$ & Cavities & Flatness & $\begin{array}{c}\text { Free } \\
\text { Aperture }\end{array}$ & $\begin{array}{c}\text { Parallelism } \\
\text { (arcsec })\end{array}$ \\
\hline 530.3 & $0.15 \pm 0.05$ & $52 \%$ & 1 & $\lambda / 4 / 45 \mathrm{~mm}$ & $45 \mathrm{~mm}$ & 30 \\
526.0 & $1.0 \pm 0.2$ & $56 \%$ & 3 & $\lambda / 4 / 25 \mathrm{~mm}$ & $45 \mathrm{~mm}$ & 30 \\
637.4 & $0.15 \pm 0.05$ & $57 \%$ & 1 & $\lambda / 4 / 45 \mathrm{~mm}$ & $45 \mathrm{~mm}$ & 30 \\
634.0 & $1.0 \pm 0.2$ & $36 \%$ & 3 & $\lambda / 4 / 25 \mathrm{~mm}$ & $45 \mathrm{~mm}$ & 30 \\
656.3 & $0.3 \pm 0.1$ & $42 \%$ & 2 & $\lambda / 4 / 25 \mathrm{~mm}$ & $45 \mathrm{~mm}$ & 30 \\
\hline \hline
\end{tabular}

Table 2.1: Technical specifications of the filters in MICA at the time of writing.

The filter box in the MICA instrument (Figure 2.3) is located in the classical mounting, i.e., in the collimated beam, just behind the Lyot stop. As a consequence of this configuration, the maximum wavelength shift, i.e., at the edge of the MICA field of view ( $\left.2 \mathrm{R}_{\odot}\right)$, and supposing that the central passband occurs at $1.05 \mathrm{R}_{\odot}(16.8$ arcmin) is according to $(2.1)$ of $\sim 0.005 \mathrm{~nm}$. The radial dependence of the central wavelength $\lambda_{0}$ of the filter transmission is shown in Figure 2.4

\section{Experimental tuning}

The tuning of the interference filter bandpass to the position of the $530.3 \mathrm{~nm}$ spectral line is a crucial point for the quality and reliability of the monochromatic corona observation. The determination of the optimal working temperature for the filters was performed by analyzing the intensity profiles in the "treated" green line emission 

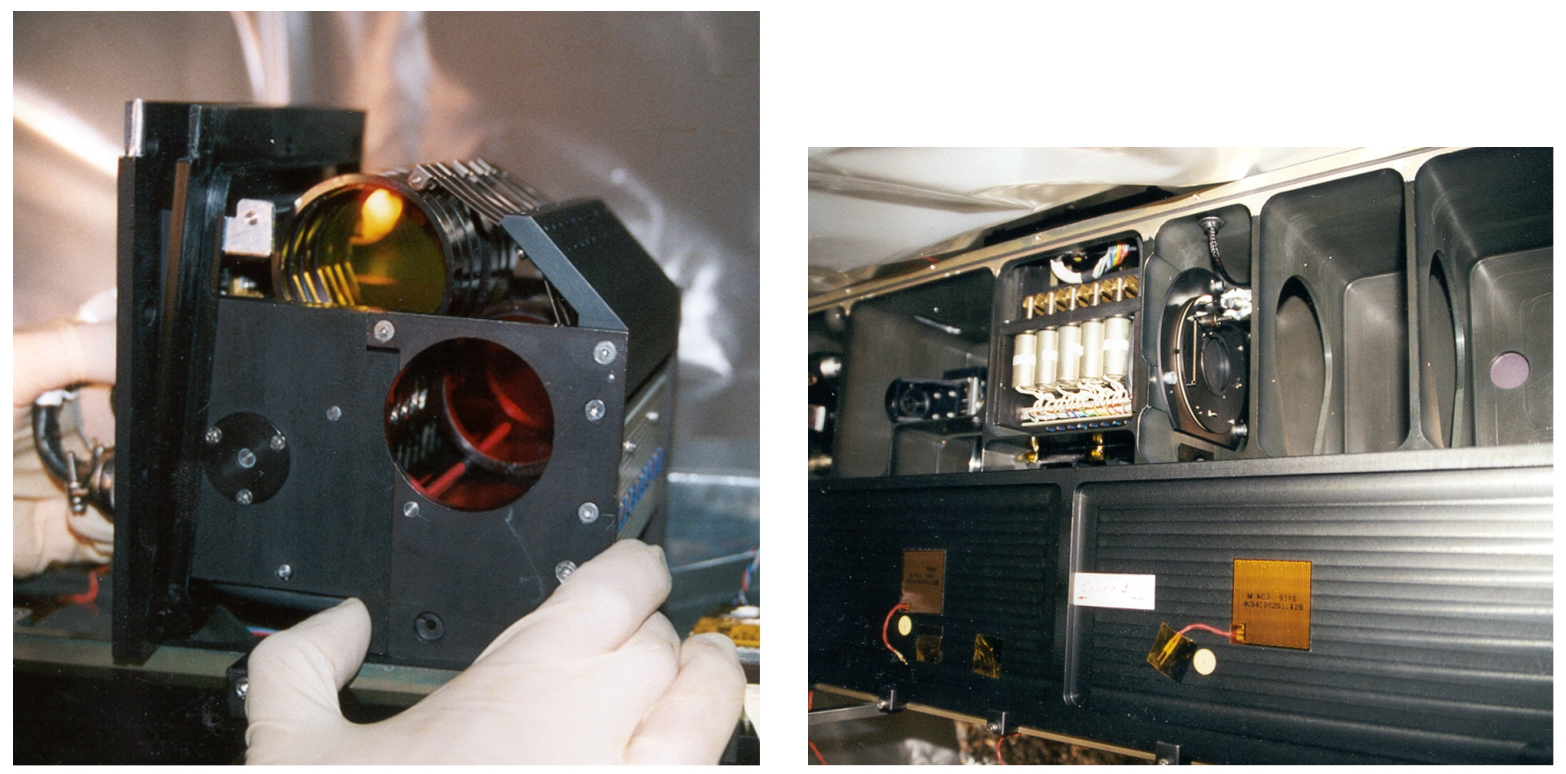

Figure 2.3: Left panel: Filters box showing the "red filter" in the position corresponding to the optical path. The right panel shows the filters box in its actual location inside the MICA instrument. The device just at the entrance of the box is the camera shutter which was relocated from its original position (Section 2.4.2).

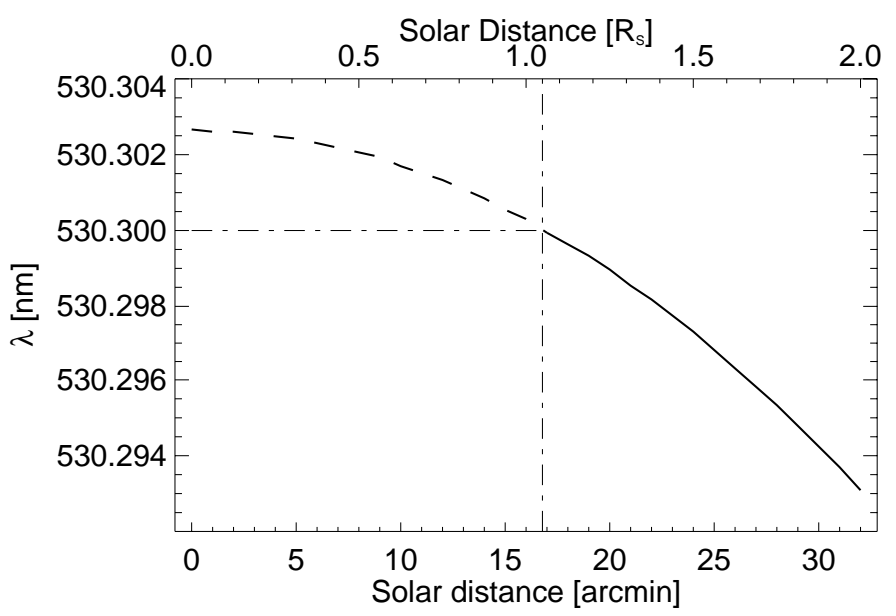

Figure 2.4: Radial dependence of the central wavelength $\lambda_{0}$ of the filter transmission according to (2.1). It is supposed that the filter is tuned at $530.3 \mathrm{~nm}$ at the inner edge of the field of view, i.e., $1.05 R_{\odot}$.

images (a detailed description of the reduction procedure is addressed in Chapter 3 ) as function of the temperature of the filter. For illustration, we show in Figure 2.5 the green line filter passband (at $1.05 \mathrm{R}_{\odot}$, solid line) relative to the real solar spectrum in the range $[529.5,530.7] \mathrm{nm}$. For comparison, the filter passband at the edge of the field of view (dashed line) is also shown. Further, the passband at $\Delta T= \pm 3 \mathrm{C}$ of the working temperature (dasheddotted-dotted line) is superimposed in the same graph. They were calculated using a temperature coefficient $K_{T}$ of $0.0168 \mathrm{~nm} / \mathrm{C}$, as reported by the manufacturer.

As can be seen in Figure 2.5, the presence of a strong photospheric absorption line nearby, i.e., at $530.23 \mathrm{~nm}$, helped in the procedure. When the filter is tuned at that wavelength, one expects to observe a black ring around 


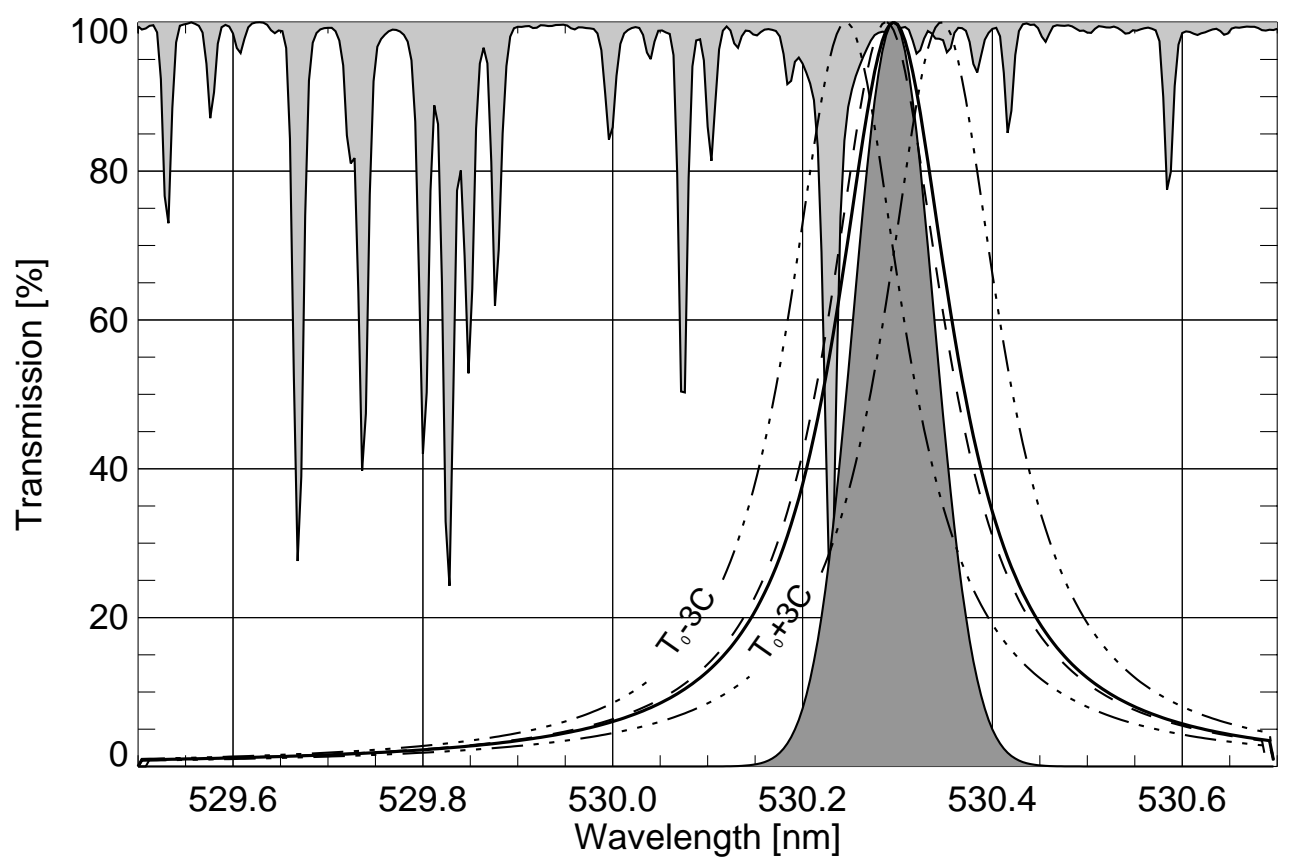

Figure 2.5: Shift of the green line filter passband with temperature and solar distance. The solid line represents the filter transmission at the temperature where the green line emission close to the limb is observed to be maximum ( $\left.T_{0}\right)$. The dashed line denotes the filter passband at the edge of the field of view, at the same temperature $T_{0}$. The dashed-dotted-dotted line depicts the filter passband close to the limb at $T_{0} \pm 3 C$ (temperature coefficient $K_{T}=0.0168 \mathrm{~nm} / \mathrm{C}$ ). For comparison the solar spectrum with the Fraunhofer absorption lines in the range [529.5,530.7] nm is plotted in light grey. The Fe XIV green line profile (see Section 3.3.7) is also shown (dark grey).

the occulter. With gradual increasing temperatures, this dark ring shifts towards the edge of the occulter until it disappears, whilst the intensity of the green line progressively increases. It was found that the temperature $\mathrm{T}_{0}$ at which the transmission is maximum is $\sim 36 \mathrm{C}$.

The same scan in temperature was repeated for the red filter, finding the best transmission at roughly the same temperature.

\subsection{The CCD camera}

The field of view of any instrument can be considered as a collection of picture elements (pixels). This field can be imaged either onto an array of detector elements in a focal plane array, or it may be imaged by a single detector or array that is scanned over the field. Because of its high sensitivity, linearity, and dynamic range, "Charge Coupled Devices" (CCD) are currently the most used devices for astronomical observations in the visible and near-infrared domains.

It is the purpose of the first part of this section, to present the main characteristics of the "Charge Coupled Devices" from a theoretical point of view. In addition, the main sources of noise present in a CCD are described 
and a method based on the noise characteristics of observations taken with uniform illumination is outlined. In view of the above information, the MICA camera is then described and a comparison of some of its technical specifications is made with values obtained based on daily observations.

\subsubsection{Theoretical background}

A CCD (Charge Coupled Device) is a photoelectronic imaging device which in principle can be described as an array of individual pixels defined by potential wells in the silicon semiconductor material. The electrons, which are generated by the absorption of photons, are collected in these pixels. By applying different voltages to the small semi-transparent electrodes on the CCD surface, the electric field defining the potential wells can be changed. By this means, a transfer of the charge from pixel to pixel onto the output node is carried out. At the output capacitance the charge of each pixel can be measured as a small voltage (typically $1 \mu V / e^{-}$). The CCD is operated by the camera electronics, which amplifies and converts the output voltages of the individual pixels into a digital number (DN), often referred to as "counts” or ADU. For details see, e.g., Mackay (1986) and Mc Lean (1989).

\section{From photons to digital counts}

In the CCD detector, the incident photons $\left(p_{i}\right)$ are converted into an equivalent output voltage $\left(v_{s}\right)$, whilst the amplification of $v_{s}$ and conversion into DN is carried out in the camera electronics. In terms of the different processes occurring in the device, the signal collected by the CCD in "counts" or DN (i.e., $N_{c}$ ) can be expressed as

$$
N_{c}=\left(p_{i} \cdot Q_{e f f} \cdot S_{\eta} \cdot A_{1}+b\right) \cdot A_{A D C}
$$

where $Q_{\text {eff }}$ is the effective quantum efficiency in $e^{-} /$photons (i.e., the number of electrons that are measured at the output of the CCD in relation to the number of incident photons), $S_{\eta}$ represents the sensitivity (i.e., $\mu V / e^{-}$ at the output node), $A_{1}$ denotes the electronic gain (i.e., amplification of the output voltage $v_{s}$ ), $b$ is the electronic signal-offset (bias) in $m V$ added to the signal to avoid negative counts, and $A_{A D C}$ is representative of the digitization process in $\mathrm{DN} / \mu V$ (i.e., signal conversion into $\mathrm{DN}$ ). In other words

$$
N_{c}=g \cdot s_{e}+B_{c}
$$

where $s_{e}=p_{i} \cdot Q_{e f f}$ is the signal in $e^{-}, g=S_{\eta} \cdot A_{1} \cdot A_{A D C}$ is the conversion factor in $D N / e^{-}$, and $B_{c}=b \cdot A_{A D C}$ is the digitized bias.

\section{Linearity and charge capacity}

The charge capacity or full well is the maximum number of electrons a single pixel can hold before spilling over. Since the p-doped channel stops at a much higher potential barrier, excess charge normally flows only into the vertical neighbour pixels. The effect of overflow of the saturated pixel is called blooming. Full well is reached when the linearity curve of the CCD signal (i.e., DN versus illumination level) shows a cut-off. Up to their saturation level, which can be slightly different from pixel to pixel, CCDs are linear devices (typically $<0.5 \%$ ). The dynamic range of the CCD is defined as the ratio of its full well capacity to the readout noise. Typical values are $10^{4}$ to $10^{5}$. 


\section{Noise sources}

Random noise places a lower limit on the smallest charge packets that can be detected and measured by the CCD. Many noise sources, both external and internal to the CCD, can contribute to this limit. These sources can be grouped into three major categories: The first group comprises the so-called intrinsic noise sources, i.e., those inherent to the CCD and processing electronics. The second category corresponds to the man made noise sources or interference noise. Examples are boundless and include radio and tv broadcast interference, ignition, power line, motor, switching systems (microprocessors), etc. The third category are sources related to natural disturbances, such as cosmic rays, lightning, etc. Among the most important intrinsic noise sources are:

Thermal noise: An stochastic, and additive source of electrons in the CCD well is thermal energy. Electrons can be freed from the CCD material itself through thermal vibration and then, trapped in the CCD well. The electrons liberated in this way are indistinguishable from the "true" photoelectrons. By cooling the CCD chip it is possible to reduce significantly the number of thermal electrons that give rise to thermal noise. The thermal noise usually appears in two types: i) a general background level resulting from thermally created electrons as aforesaid, that is quite low, and ii) locally "hot" pixels that display vastly higher rates of thermal electron creation. The thermally generated charge accumulated is proportional to the exposure time and governed by Poisson statistic, where the rate parameter is an increasing function of temperature. Hence, taking into account the thermally generated electrons, Equation 2.3 can be rewritten as

$$
N_{c}=g \cdot\left(s_{e}+d_{e}\right)+B_{c}
$$

where $d_{e}$ represents the number of thermally generated electrons and $g, s_{e}$, and $B_{c}$ are the same as in Equation 2.3.

Readout noise (e $\mathbf{e}^{-}$/pixel): It is the noise caused during the electronic readout process (see, e.g., Janiseck, 1991). It is dependent on three factors: i) white noise and ii) flicker (1/f) noise associated with the on-chip output amplifier, and iii) the sensitivity of the sense mode (it represents the final collecting well for all charge packets). Since the readout noise has no associated reduction step for its minimization, it becomes an irreducible contribution to the error budget. Nevertheless, readout noise can be reduced to manageable levels by appropriate readout rates and proper electronics.

Digitization noise: This is not a classical noise source, however, the conversion carried out in the ADC (Analog to Digital Converter) must be accounted for in statistical arguments. Poisson statistical arguments are valid after converting the DN back to electrons (equal to number of photons detected). The presence of the conversion factor $q=1 / g$ (in units of $e^{-} / \mathrm{DN}$ ), usually referred as "gain" of the system, between the analogue measurement of charge in a CCD pixel, and its digital representation is a minor complication to consideration of signal and noise levels. However, it is important to remember that readout noise is usually quoted in electrons, directly relatable to the number of detected photons, but that the signal level associated with each pixel is scaled down by $q$. 
Besides the intrinsic noise sources mentioned above, the most basic noise source, of an irreducible nature, is that of photon noise. The physical signal the camera has to detect is based upon light; thus, its quantum nature plays a significant role. The photon noise arises from the fundamentally statistical nature of photon production. In a given pixel for two consecutive but independent observation intervals of equal length $T$, the number of photons will not be identical. The probability of obtaining $n$ photons in a measurement of $T$ seconds length is known to follow a Poisson distribution ${ }^{8}$. It is critical to understand that even if there were no other noise sources in the imaging chain, the statistical fluctuations associated with photon counting over a finite time interval $T$ would still lead to a finite signal-to-noise ratio $(S N R)$. The three traditional typical assumptions about the relationship between signal and noise do not hold for photon noise, i.e., i) photon noise is not independent of the signal, ii) photon noise is not Gaussian, and iii) photon noise is not additive.

\section{Determination of the camera gain and readout noise}

The study of the noise characteristics of a CCD camera allows the determination of both the conversion factor $g$, and readout noise of the camera. The method I follow is basically that described by Prabhu (1992). In a unit integration period, a certain amount of electrons $\left(N_{e^{-}}\right)$is accumulated in a given pixel and converted by the camera electronics into DN, i.e., $N_{c}$ (see equations 2.2 and 2.4). Equation 2.4 can be rewritten as

$$
N_{c}=B_{c}+g \cdot d_{e}+g \cdot f \cdot s_{e}=B_{c}+d_{c}+f \cdot s_{c}
$$

where $B_{c}$ is the digitized bias, $d_{c}$ is the thermally generated charge (in $\mathrm{DN}$ ), and $f \cdot s_{c}$ is the number of counts (i.e., DN) generated by the number $p_{i}$ (see Eq. 2.2) of incoming photons. The factor $f$ was introduced to account for the individual quantum efficiency of each pixel, and it is defined such that its mean value is unity.

For uniform illumination (i.e., a flat field), the variance $\left(\sigma^{2}\right)$ of the number of observed counts in the image can be written as

$$
\sigma^{2}=g^{2} \cdot B_{e}^{2}+g \cdot\left(<d_{c}>+<s_{c}>\right)+\sigma_{f}^{2} \cdot<s_{c}>^{2}
$$

where $B_{e}$ accounts for the base-level noise in electrons and equals the sum in quadrature of the readout noise and noise from other signal-independent sources (Newberry, 1991). The brackets $\diamond$ were used here to denote mean values. In deriving (2.6), we have assumed that the noise is Poissonian in both the signal electrons and the thermally generated electrons (i.e., in $s_{e}$ and $\left.d_{e}\right)^{9}$. Since most CCD currently available have very low thermal charges at the working temperature, the term $g \cdot d_{c}$ in (2.6) can be neglected. Therefore,

$$
\sigma^{2}=g^{2} \cdot B_{e}^{2}+g \cdot s_{c}+\sigma_{f}^{2} \cdot s_{c}^{2}
$$

\footnotetext{
${ }^{8}$ A Poisson distribution is characterized by

$$
P(n, T)=\frac{(\rho T)^{n} \exp ^{-\rho T}}{n}
$$

where $P(n, T)$ denotes the probability of obtaining $n$ photons in a time interval $T$, and $\rho$ represents the photon rate measured in photons per second.

${ }^{9}$ For the derivation of (2.6), the estimation of the noise in both the signal and thermally generated charge was calculated as follows:

- $\sigma_{d_{c}}^{2}=\sigma_{g \cdot d_{e}}^{2}=\left(g \cdot \sqrt{d_{e}}\right)^{2}=g^{2} \cdot d_{e}=g \cdot d_{c}$

- $\sigma_{f \cdot s_{c}}^{2}=\sigma_{f \cdot g \cdot s_{e}}^{2}=g^{2} \cdot s_{e}+g^{2} \cdot s_{e}^{2} \cdot \sigma_{f}^{2}=g \cdot s_{c}+s_{c}^{2} \cdot \sigma_{f}^{2}$ having made use of $\langle f\rangle=1$
} 
where we have dropped for simplicity the brackets $<>$ denoting mean values.

A quadratic fit to (2.7) yields all the constants., i.e., $g$ and $B_{e}$, by using a set of flat field images at different exposure levels (or equivalently different exposure times). In practice, the accuracy of the method can be enhanced by the reduction of the third term of Equation (2.7), which arises from the pixel to pixel sensitivity variations and the non-uniformity of illumination in the flat field. In order to reduce the contribution of the third term (effect of flat field noise), I employed the method by considering the division of two equally exposed flats as suggested by Mackay (1986). If $S$ indicates the mean number of counts in both flat fields (bias, dark, and flat corrected), it can be easily demonstrated that Equation 2.7 turns to be:

$$
\sigma^{\prime 2}=g^{2} \cdot B_{e}^{2}+g \cdot S
$$

where

$$
\sigma^{\prime 2}=\frac{S^{2}}{2} \sigma_{S_{1} / S_{2}}^{2}
$$

$S_{1} / S_{2}$ is the image resulting of dividing the first flat field by the second one, and $\sigma_{S_{1} / S_{2}}^{2}$ represents the variance of it. In this way, the value of $g$ can be accurately determined by means of a least square polynomial fit, although the determination of $B_{e}$ would still be inaccurate since the linear term dominates.

The base-level noise $B_{e}$ includes, in addition to the readout noise $R_{e}$, noise due to pick up from external signals and truncation noise $\left(T_{e}\right)$ due to digitization of the analog data from the CCD chip. The contribution to the base-level noise due to truncation (i.e., the "quantization noise" described above) can be estimated according to Newberry (1991) as follows:

$$
T_{e}^{2}=\left(q^{2}-1\right) / 12
$$

where $q=1 / g$.

\subsubsection{The MICA camera}

The field of view of the instrument ranges from $1.05 \mathrm{R}_{\odot}$ to $2.0 \mathrm{R}_{\odot}$. It is covered by a $1280 \times 1024$ square pixels Peltier-cooled CCD camera with 12-bits AD conversion (for a more complete technical description of the camera, see Table 2.2). One pixel (16 $\mu \mathrm{m}$ length) subtends 3.7 arc sec on the solar disk, resulting in two-pixel spatial resolution of about $8 \mathrm{arc} \mathrm{sec}$. The working temperature of the CCD is kept constant at $-30 \mathrm{C}$ using a Peltier element which, in turn, is kept at a constant temperature environment by a cooling system that keeps water circulating around the CCD chip at $5 \mathrm{C}$.

The built-in camera shutter (located just in front of the CCD chip) was disabled, a new one being mounted before the Lyot stop $A_{1}$ (see right panel on Figure 2.3). The reason is that at the original position, the shutter acts such that the light coming from elements far from the optical axis is attenuated earlier than the light coming from objects near the optical axis, while in front of the Lyot stop the light coming from everywhere is attenuated in the same manner. 


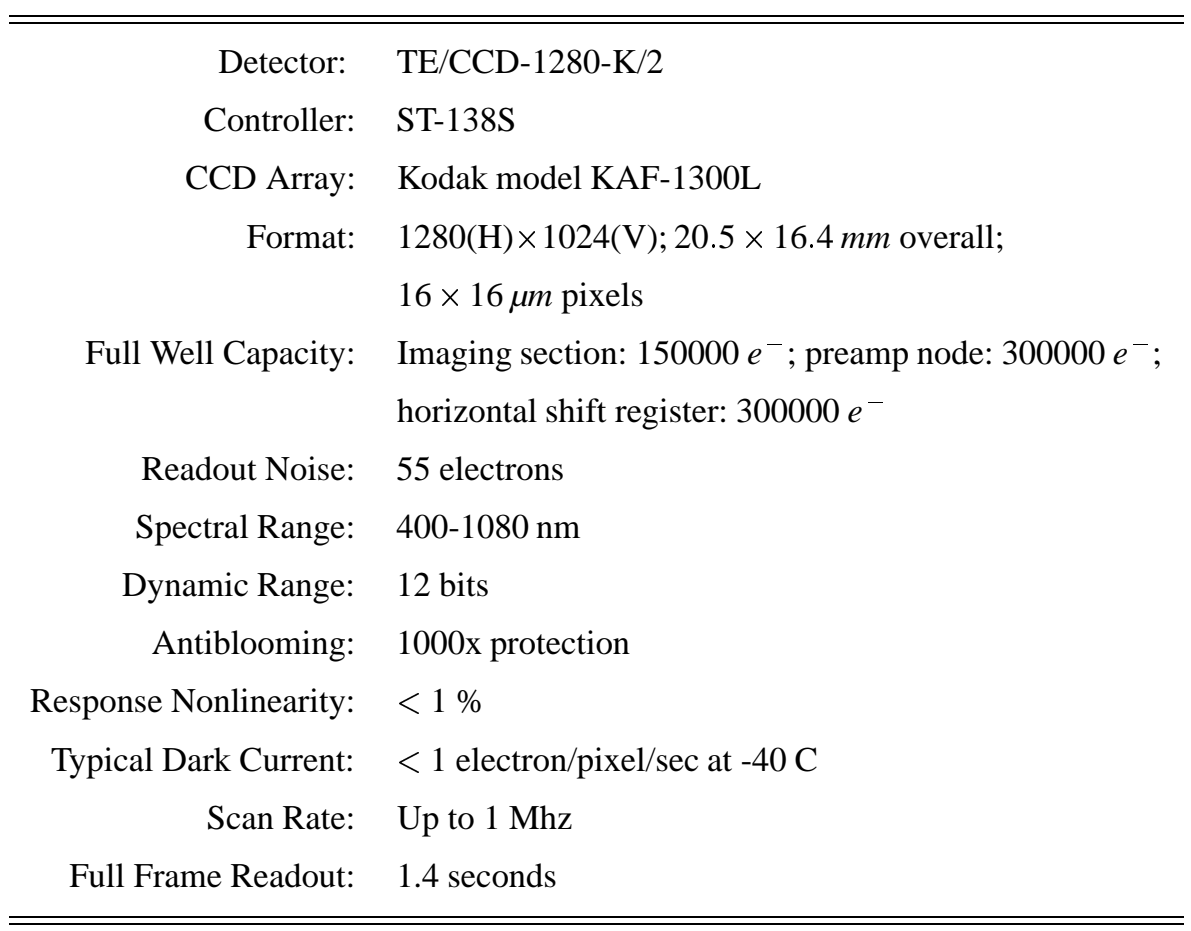

Table 2.2: The MICA camera: Technical specifications and performance characteristics as given by the manufacturer.

\section{Camera gain and base-level noise}

For practical purposes, the base-level noise $B_{e}$ is daily estimated in DN by measuring the histogram spread of the image resulting from the difference of two dark images with very short exposure time (differencing removes bias contribution). For this estimation is assumed that $B_{e}$ follows Gaussian statistic. In this way, the spread in the resulting difference image is $\sqrt{2}$ larger than the $B_{e}$ noise in a single image. The value found for $B_{e}$ is $1.5 \pm 0.1$ counts.

The camera gain, i.e., $q=1 / g$, in $e^{-} /$count is experimentally derived by using (2.8) with the value $B_{e}$ determined as mentioned above. Flat fields taken under different illumination levels (bias and dark corrected) are used for that purpose. The experimental value of the camera gain is found to be $41.0 \pm 0.5 e^{-} /$count. Hence, the base-level noise in $e^{-}$is $\sim 60 e^{-}$. The contribution to the base-level noise due to the truncation error (Eq. 2.9) is $\sim 12 e^{-}$.

\section{Bias and thermally generated charge}

The camera offset, i.e., bias, is estimated by plotting the mean number of counts of dark images taken with different exposure times as function of the exposure time, and fitting them with a straight line. Extrapolation to exposure time of $0 \mathrm{sec}$ gives an estimate of the bias value (see Section 3.4). In this way, the camera offset is found to be $\sim 111$ counts.

The slope of the aforementioned plotting multiplied by the gain of the camera is a good estimate of the thermally generated charge per unit time, i.e., the so-called dark current. Daily estimates of such a value give for the 
dark current a value of less than 1 electron per second at $-30 \mathrm{C}$.

A summary of the average values obtained experimentally for the MICA camera are shown in Table 2.3.

\begin{aligned} & \hline \hline Camera gain $q: \sim 41 e^{-/ D N} \\ &$ Readout Noise: $\sim 48$ electrons \\ & Truncation Noise: $\sim 12$ electrons \\ & Bias: $\sim 4550$ electrons (111 counts) \\ & Dark Current: $\sim 0.9 e^{-/ \text {sec } \text { at }-30 \mathrm{C}} \\ &$\hline \hline\end{aligned}

Table 2.3: Parameters of the MICA camera determined experimentally (as obtained along one year). For details see text.

\subsection{Auxiliary devices}

For the automatic operation of the telescope, the system needs of a set of auxiliary devices i) to keep automatic tracking of the Sun, ii) to detect the actual weather conditions in the site, iii) to control the temperature of the filters and canister of the telescope, iv) to control the camera operation and filters mechanism, etc. Without going into any technical detail, the main components that help the telescope to operate as a stand-alone system will be briefly described.

\subsubsection{The tracking system}

The telescope is mounted on a German equatorial mounting driven by two geared stepper motors that control its movement in declination and right ascension. Since the telescope could not be perfectly polar aligned ${ }^{10}$, it suffers a small drift in declination during the day. Further, the telescope tube may flex a little as it tracks the Sun across the sky, the electricity running the drive motor may be slightly irregular, and the gear box may suffer from mechanical deficiencies. Since the atmospheric refraction varies as the Sun's altitude changes, the Sun's real position is altered. And the slowest component of atmospheric seeing or turbulence, can make the solar disk creep around slightly with a characteristic time of many seconds. Therefore, an electronic drive corrector that speeds or slows the telescope's drive motors is necessary. This allows fine guiding in right ascension (east-west), where the most frequent corrections are needed as well as in declination (north-south). Thus, the tracking controlling system with its associated electronic controls the operation of the stepper motors and hence the movement of the telescope during the observation time according to the information retrieved by the so-called Coarse and Fine Pointing mechanisms.

\footnotetext{
${ }^{10}$ Alignment of the right-ascension axis parallel to the Earth's axis of rotation
} 

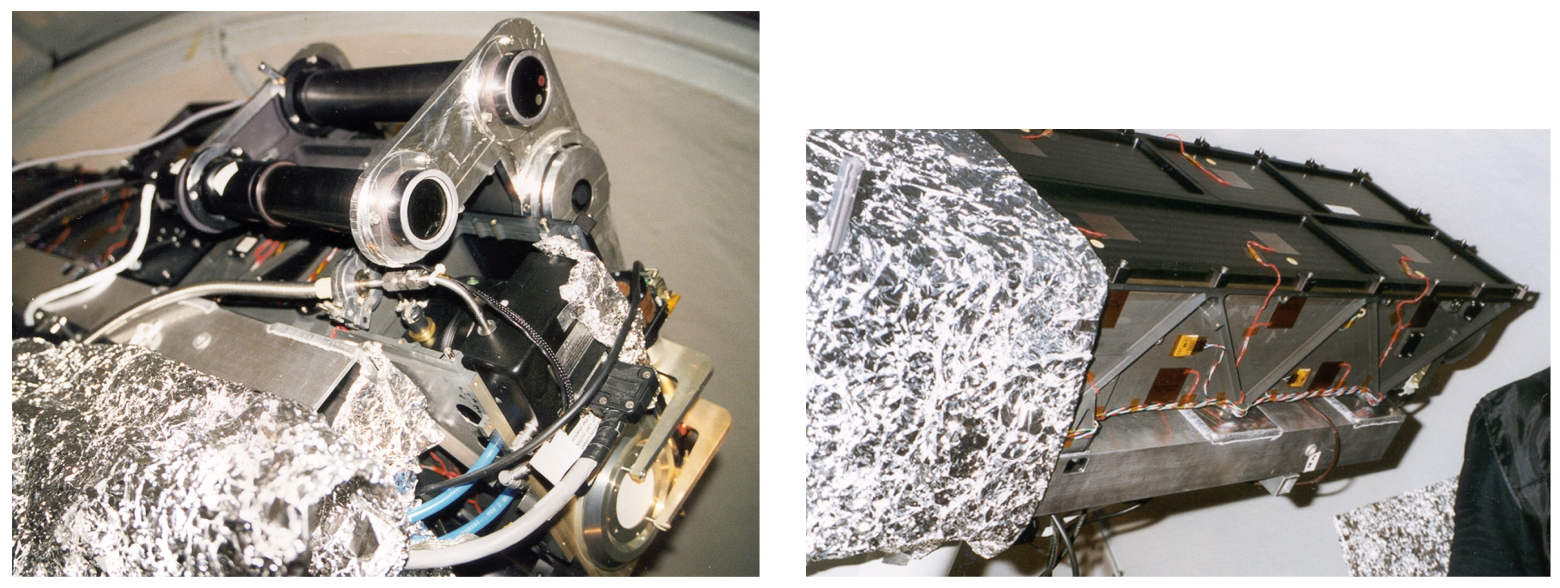

Figure 2.6: Left panel: Front view of the MICA instrument. Part of the thermal canister (see Section2.5.3) was removed to allow the view of the CCD camera back side. The tube on the left of the camera holds the Coarse Pointing mechanism (Section 2.5.1), the next one (just at the top of the panel) contains the "Sun Tester" device, and the closest one to the door (i.e., that with the silvered front) holds the "Sky Tester" device (Section 2.5.2). Right panel: Part of the thermal canister (aluminium foil) and set of resistances uniformly distributed around the structure (Section 2.5.3).

\section{The coarse pointing}

For the coarse pointing of the Sun, a little external telescope mounted parallel to the main structure is used (see left picture on Figure 2.6). The incoming solar light is collected by a "Photo Quadrant analog detector" after passing through a neutral density filter. The associated electronic of the detector transforms the incoming photons in four electronic analog signals, i.e., $\mathrm{mV}$. In an intermediate device, the so-called microcontroller ${ }^{11}$, the four analog values are digitized and then transformed into binary signals according to a certain threshold value. In other words, if the signal in $\mathrm{mV}$ is above a certain threshold the value for the respective quadrant is set to 1, while below that threshold the value is set to 0 . The control software corrects the position of the telescope in such a way to obtain a value of 1 in all four quadrants.

\section{The fine pointing}

For fine pointing, the light from the solar disk enters into an optical system after passing through the inverse occulter $\mathrm{M}_{2}$, where a Sun sensor consisting of four photodiodes (a "Photo Quadrant analog detector") is located (see Figure 2.7). In this optical system, the sunlight is reflected by the diagonal mirror $\mathrm{M}_{4}$ and collimated by the collimating lens $\mathrm{C}$. The collimated beam is then further reflected by the mirror $\mathrm{M}_{5}$ onto a telelens TL, which focuses the light beam onto the detector. Before reaching the detector the light beam is attenuated with the help of a neutral density filter ND. The four diodes of the detector detect the position of the solar disk, and hence provide information on instrument pointing relative to the solar disk. Each quadrant gives an analog signal in $\mathrm{mV}$

\footnotetext{
${ }^{11}$ The signals obtained by most of the auxiliary devices are digitized in an auxiliary electronic device called microcontroller. Since the ADC converter is 8 bits, the minimum increment that can be detected is $\delta \varepsilon=\operatorname{signal}(m V) / 2^{\circledR}$, i.e., $\delta \varepsilon$ defines the sensitivity with which the variations in the respective signals can be detected.
} 


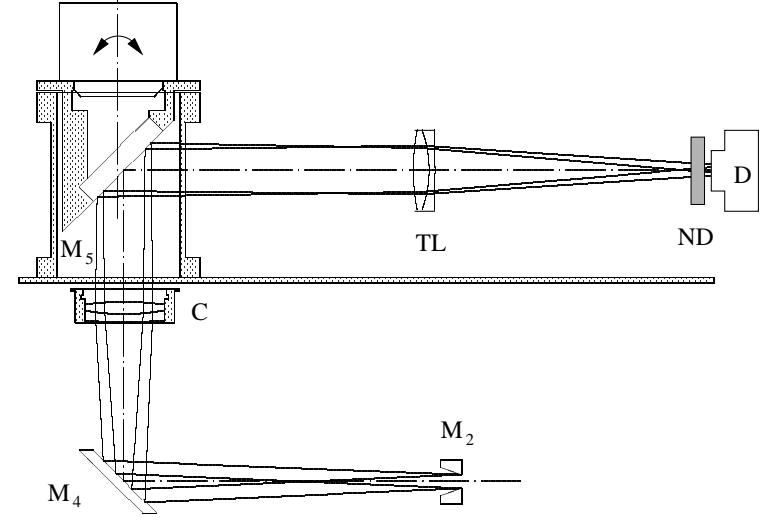

Figure 2.7: Light path for the Fine Pointing Mechanism. $M_{2}$ : Inverse occulter, $M_{4}$ : diagonal mirror, $C$ : collimator, $M_{5}$ : semitransparent mirror, TL: telelens, ND: neutral density filter, $D$ : four-quadrant detector.

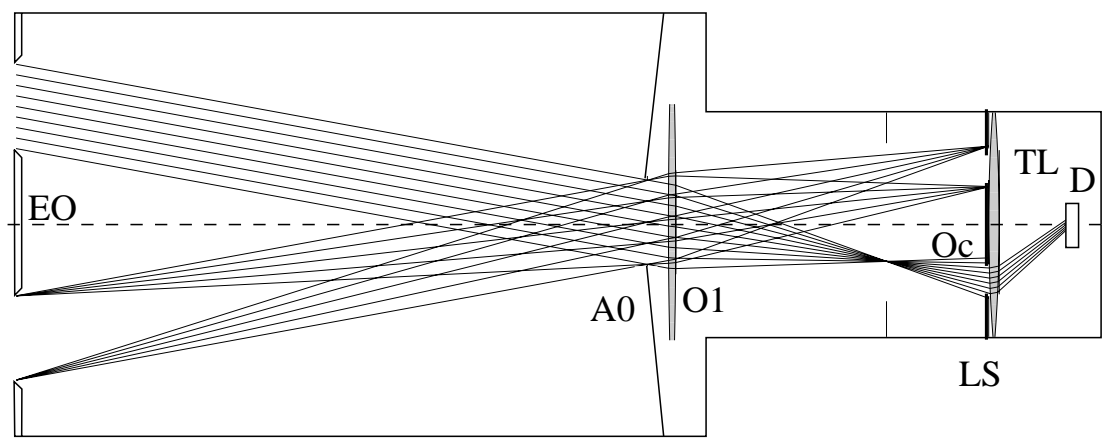

Figure 2.8: Sky tester as an externally-occulted coronagraph. EO: External occulter, A0: entrance aperture, O1: objective lens, Oc: internal occulter, TL: transfer lens, D: detector.

proportional to the incoming light. The difference between values of opposite quadrants shall be zero if the solar disk is well centered. Therefore, this pair of values is continuously given back to the controlling software for the system feedback.

\subsubsection{The sky and Sun brightness measuring devices}

The automatic operation of the instrument requires a device capable of continuously monitoring the sky, to provide the necessary information about the actual observing conditions to the controlling program. This information is necessary in order for the program to take the correct line of action, independently of whether the instrument is taking images or not.

In its simplest form an instrument to measure the sky brightness around the solar disk is but a little externallyocculted coronagraph (Figure 2.8). It consists of an appropriate detector and the associated optics, a system that allows to view a specific solid angle around the solar disk, the Sun being covered by an occulter. It was designed such that the detector output current (voltage) is a linear function of the light power on the detector. For a detailed description of such a device, which we call sky tester, the reader is referred to Epple (1997).

Concerning the Sun's brightness measurement, another auxiliary little telescope with the appropriate detector (the Sun tester) and a neutral density filter to attenuate the incoming sunlight, observes the solar disk. The output 
of that detector in $\mathrm{mV}$ is proportional to the Sun's brightness. The detector is a planar Silicon PN photodiode in a hermetically sealed short TO-5 case, especially designed for high precision linear applications (the commercial description is BPW 20R).

A picture showing the position of the Sky and Sun tester telescopes is shown in the left panel of Figure 2.6.

\section{How do the sky and Sun tester help to the automatic operation?}

Let us first observe Figure 2.9 to understand their behaviour under different sky conditions. The sky tester is very sensitive to minor changes in sky brightness. The intensity level registered by the sky tester (which is function of the air mass, dependence not important for the present discussion) is low when the sky has coronal quality. Therefore, one is tempted to say that if the intensity level is below a certain threshold the telescope is in conditions of observing. Unfortunately, if a thick (dark) cloud is above the instrument the signal is low, too (see, e.g., panel corresponding to July 25, 1998 in Figure 2.9). The result is that with only this auxiliary instrument no decision can be taken to decide whether the observing conditions (sky related) are appropriate or not for observing. With the help of the Sun tester this problem is overcome. When the solar disk is observed to be free of clouds the registered intensity by the Sun tester is maximum (again air mass depending). In this way, the sky observing condition is fulfilled when the sky tester reports a value lower than a certain threshold and the Sun tester reports a value larger than another certain threshold. The threshold values were fixed experimentally in El Leoncito, according to the experience gained. For the Sun tester, the threshold is fixed to $2.2 \mathrm{mV}$, while for the sky tester it was fixed to $0.95 \mathrm{mV}$ until December, 1997. At the time of writing, it is set to $1.3 \mathrm{mV}$, although observations with thresholds up to $3.0 \mathrm{mV}$ were also carried out. The biggest problem found is that for high sky tester values the sky is usually very variable, too. Therefore, with those high threshold values, the observation of the emission solar corona signal was not reliable ${ }^{12}$.

\subsubsection{Temperature controlling}

Thermal stabilization of the instrument (i.e., of the box and filters) is required in order to i) keep the central filter passbands at the right value, ii) prevent the degradation of the optical elements due to condensation of gaseous contaminants, and iii) reduce to a minimum the presence of thermal gradients inside the instrument independently of external conditions. Therefore, electrical heaters were placed at appropriate locations all over the telescope. The actual temperature of the structure and filters is monitored and controlled through the microcontroller.

\section{Structure}

The MICA optical system is enclosed in a lightweight thermal canister which maintains thermal stabilization of the telescope all along the day during the four seasons. In addition, a set of resistors is uniformly distributed on the walls of the instrument in order to maintain a constant working temperature and avoid mechanical deformations of the structure during observations due to temperature changes as well as turbulence insight the telescope due to

\footnotetext{
${ }^{12}$ For a detailed discussion of the effects of the sky variability on the images see Section 3.3.5.
} 

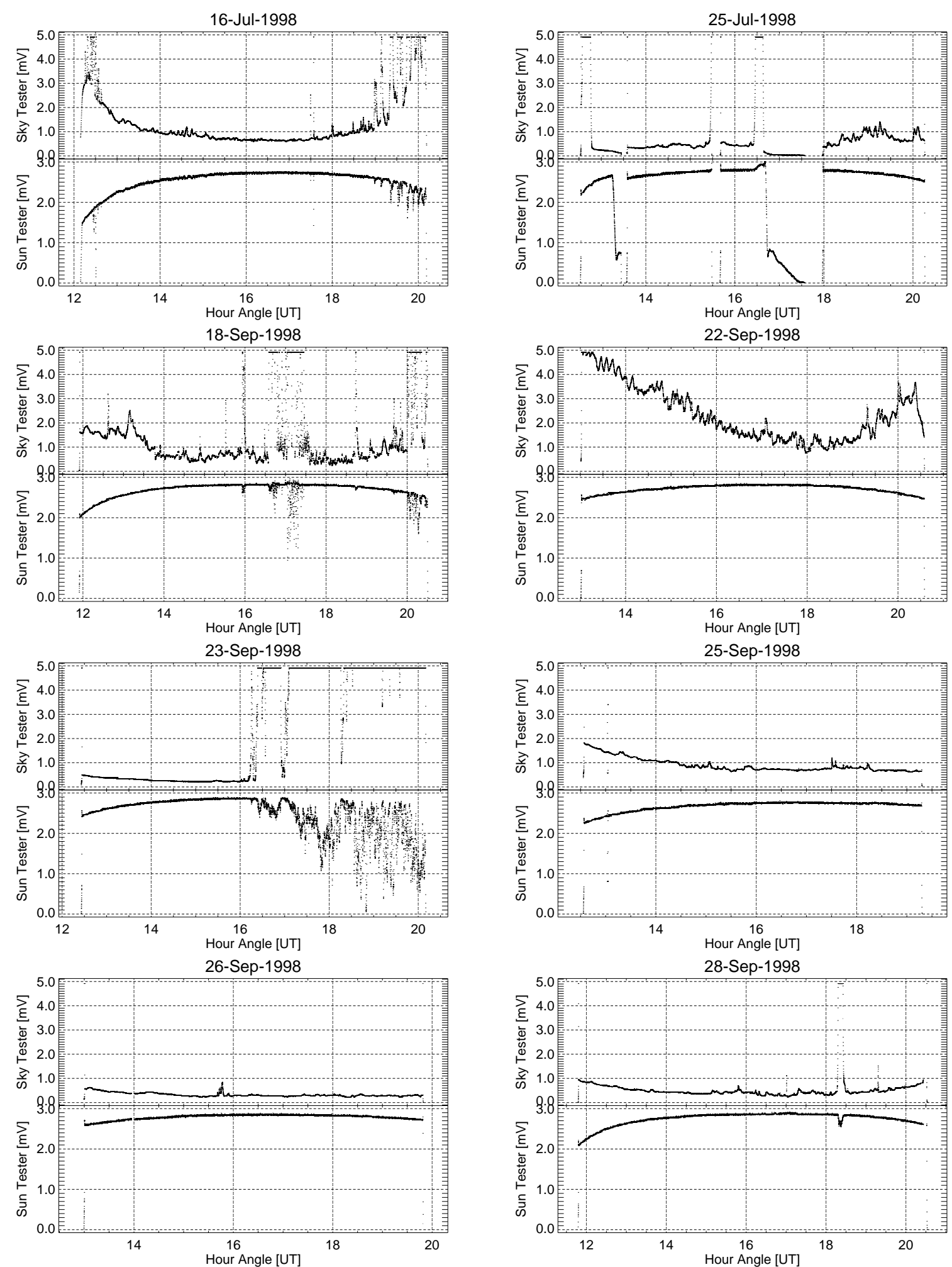

Figure 2.9: Examples of sky and Sun brightness intensity as recorded by the sky and Sun tester devices. The presence of "dark" clouds can be inferred by low signals in both instruments (e.g., on July 25, 1998). Thin clouds (e.g., the so-called "cirrus") are discerned because of the high values reported by the sky tester with the corresponding decrease of the Sun's brightness (e.g., on July 16 at the end of the day, September 18 mainly between 16:00 UT and 18:00 UT and after 20:00 UT, and September 23, 1998 after 16:00 UT). 
temperature gradients (see right panel on Figure 2.6).

Originally, the temperature system was designed to keep the temperature of the structure at $\sim 30 \mathrm{C}$. This value is close to that required for the filters according to the manufacturer specifications. Unfortunately, analysis of the position of the occulter in the images revealed a strong correlation between the position of the center of the occulter and the duty cycle ${ }^{13}$ of the temperature controlling system. For that reason we had to experimentally find the maximum temperature allowed for the structure such that no variations in the occulter position due to the heating cycle were found (the position variation must be less than 1 pixel). An actual working temperature of $\sim 26 \mathrm{C}$ was finally chosen.

\section{Filters}

As explained in Section 2.3.1, the filters have to be tuned to the right wavelength by adjusting their working temperature. For that reason, the filter box is separately temperature controlled. The control mechanism also consists of a set of resistors, which are placed in the filter box. The system is designed to work at a temperature of $\sim 35 \mathrm{C}$, i.e., at a temperature slightly higher than that of the structure. Note that the system cannot be cooled down. This issue can become rather problematic during summer time, when the ambient temperature may reach $\sim 40 \mathrm{C}$ at local noon.

\subsection{System integration: An intelligent system}

The instrumentation of the whole MICA system allows, among other things, an extremely sensitive and accurate determination of the sky transparency and scattered light levels. Amount and spectral distribution of the sunlight scattered in the terrestrial atmosphere are sensitive indicators of the amount and type of atmospheric pollution. For obtaining valid and useful data, a continuous operation of the system regardless of the atmospheric conditions is required. This would not be possible without a control software in charge of commanding the different subsystems and of taking the corresponding decisions according to the variable atmospheric parameters continuously recorded by them. The advantage of such a system is that the presence of an observer at the site is not necessary. For safety purposes however, a housekeeper has to be present. He is in charge of opening and closing the dome, as well as taking care in case of unexpected problems. The system is capable to decide when to start the observations following a predefined observational sequence, when to stop them in case of adverse external factors, etc. In the rest of the chapter, I briefly describe how the system works and discuss the flow diagram of the controlling program which I specifically designed and developed.

\subsubsection{Block diagram}

\footnotetext{
${ }^{13}$ The regulation system of the temperature was designed to work with an error margin of $\pm 0.2 \mathrm{C}$. Even with this error margin, the mechanical deformation suffered by the structure still produced changes in the occulter position.
} 


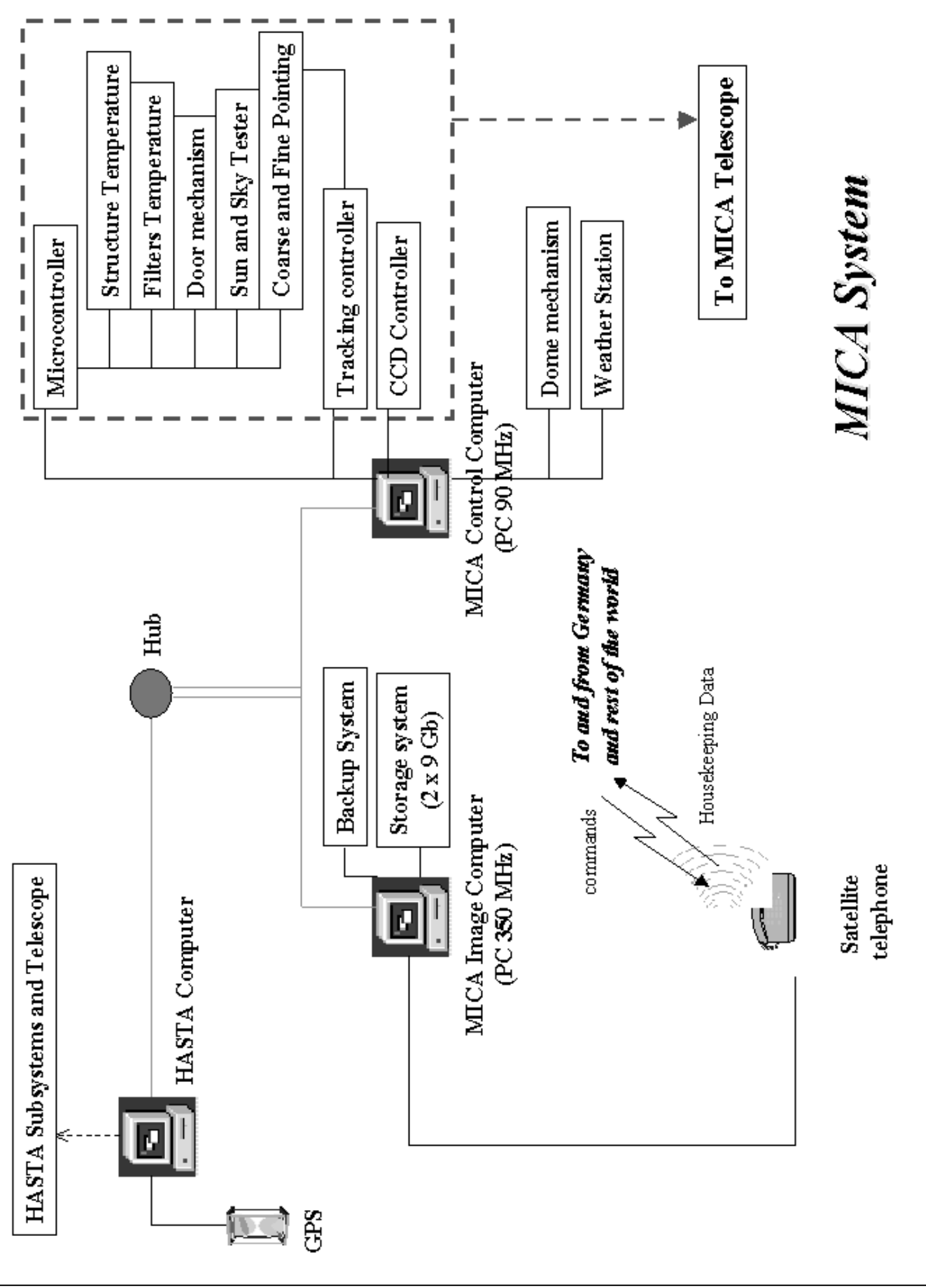

Figure 2.10: Block Diagram of the MICA system. Both MICA and HASTA (Appendix C) computers are connected through the same Ethernet network in order to share resources, e.g., a GPS (Global Position System) device. The GPS is used to provide the exact time of the observations. 
In Figure 2.10 the block diagram of the whole MICA system is shown. The system is controlled by two computers. The first one, heart of the system, is the so-called "Control Computer". At the time of this writing, it is a PC $90 \mathrm{Mhz}$ running under Windows 95. This computer is in charge of controlling the different parts that conform the whole system. Several software modules, specifically designed under $\mathrm{C}++$, command the electronic components. The commands are sent according to both the requirements of the observation routine actually running and the information received from the different components about external and internal operating conditions such as, e.g., sky conditions and temperature of the filters respectively. The second computer (i.e., the "Image Computer"), a 350 Mhz PC running under windows NT, is in charge of the real time processing of the images taken by the telescope. Data communication between both computers is carried out by an Ethernet Network.

\subsubsection{How does the system work?}

The flow diagram indicating the main steps is shown in Figure 2.11. A default operation routine is normally running. This routine continuously checks the time system ${ }^{14}$ and, if the time is such to start observations (according to a default observation routine), the module that commands the tracking controlling subsystem takes control. First, it moves the telescope off its sleeping position and points it towards the Sun by using the ephemerides. After this position is reached, the Coarse Pointing is connected. Once the Coarse Pointing is successful and provided the sky conditions are appropriate, the entrance door is opened and the Fine Pointing is connected. Once the Fine Pointing is achieved, the observation routine starts, i.e., the filter box and the CCD camera are operated by the corresponding low level modules. After each exposure, the intensity level of the image obtained is analyzed. The exposure time of the respective image is automatically corrected in case of need, the image being taken again with the new exposure time. During the whole process, every 5 seconds in average, all the subsystems are checked (fine pointing status, door status, structure and filter temperature, dome position, etc.) and the resulting housekeeping data is stored in the "Control Computer". The feedback in the system allows the self-correction of wrong values. If one of the "critical" conditions (i.e., wind speed, door position, or sky or Sun brightness) is not fulfilled, the observation stops, the entrance door is closed, and the telescope enters the so-called "waiting mode". In this mode, all the systems continue being checked as before but no images are taken, with the entrance door closed. Once the observing conditions are reestablished, the system automatically resumes operation. At a specified ending time, the telescope stops observation and, after closing the door, is automatically brought to the sleeping position.

The most serious problem we have to face with ground-based coronagraph observations is the sky variability. The first thought was to develop an algorithm to automatically check if the sky brightness kept constant during the observation sequence. If this condition was not fulfilled, the images would be discarded. Such an algorithm was implemented, but we realized that too many images were discarded for not fulfilling this condition. Thus, we decided to keep every image despite the variability of the background. As a result, the calibration turned out to be not very accurate in those cases subjected to great variability, as will be shown in Chapter 3. However, tracking

\footnotetext{
${ }^{14}$ The accuracy of the time provided by the computer is warranted by a GPS (Global Position System) device (see Figure 2.10).
} 


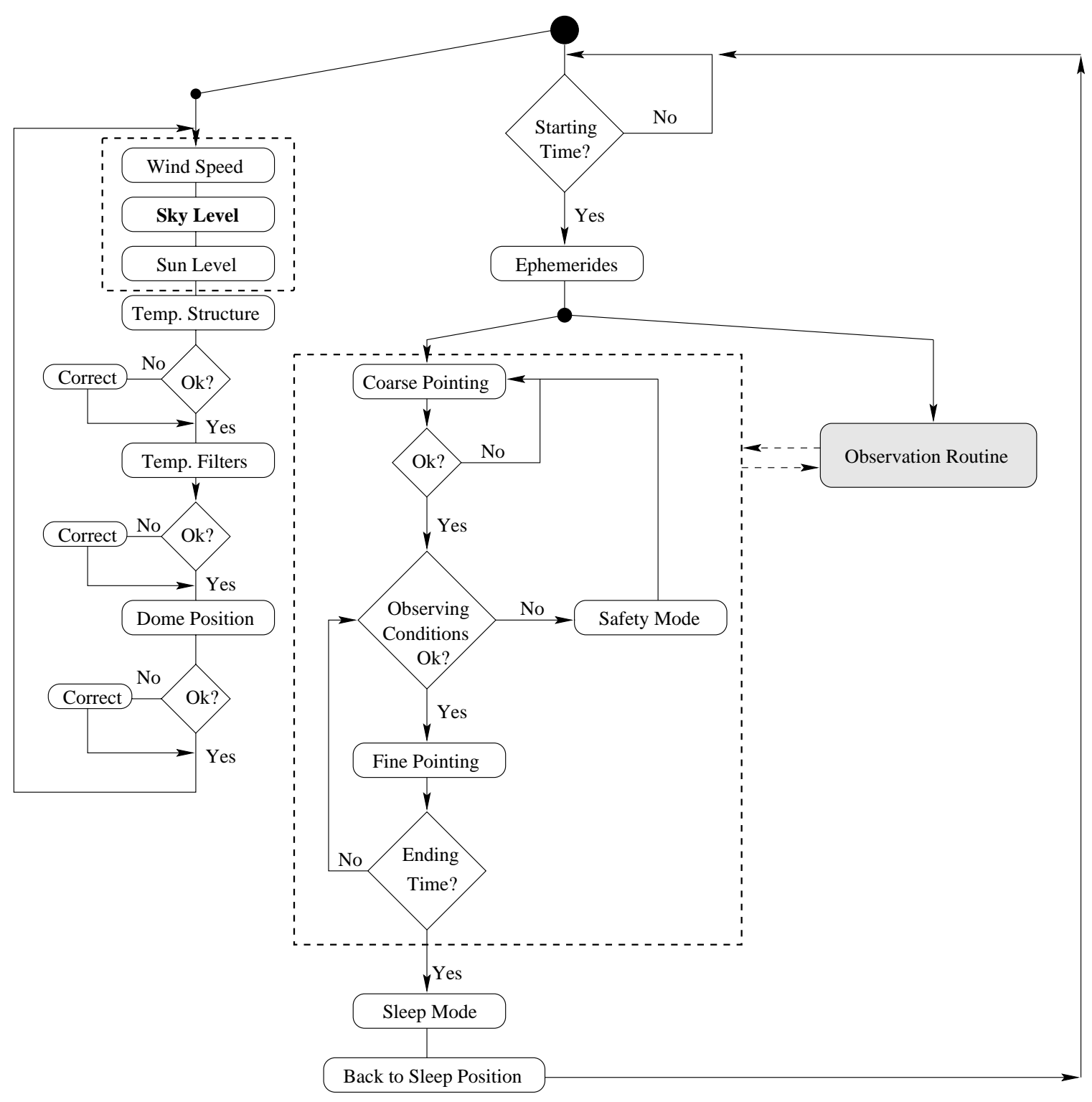

Figure 2.11: Flow diagram showing the default operation mode of the MICA system (Figure 2.10). For details see text.

of the morphology of dynamical events became possible regardless of the flickering background as the results will show. The other constraint we have to face is the presence of small seeds flying around above the telescope during spring time at the site, due to the presence of Aspen trees in the valley. Figure 2.12 shows an example of the effect of the "flying" seeds on the images. Again, an algorithm was developed to discard affected images, but for the reasons aforementioned it was finally decided not to use it. When viewing the images as a movie, morphology of dynamical events is still well trackable, like when viewing a scene through a dirty window. The image background continuously and randomly changes, while the selected events follow a trend.

While the "Control Computer" manages the operation of the telescope, another software program specifically 
designed running in the "Image Computer" continuously checks for the presence of completed sequences. Once a sequence is completed (as will be explained in more detail in Chapter 3, a sequence comprises $n$ images, i.e., the on-line and off-line images as well as the respective flats for that sequence) the reduction software (Section 3.4) proceeds to reduce the raw data and show in almost real time what is actually happening in the emission line corona. At the end of the day the raw data are automatically backed up for later shipment.

\subsubsection{The Graphical User Interface (GUI)}

Although the system is prepared to run as a stand-alone system, a complete and easy to use graphical interface for monitoring and manual control had to be designed. It was developed under IDL 4.0 and works as the link between the low level modules commanding the electronic components and the operator/user. It allows not only monitoring of the system but also controlling and commanding of the whole system, enabling the setting of filter and structure temperatures, changing of filters, tracking controller, dome movement, etc. in case of need (either for not standard observation routines or correction of problems). Off-line development and allowance of manual execution of new observation routines is another feature of the GUI which allows the user to prepare specific observation programs.

\subsection{Concluding remarks}

The MICA telescope, a small, low scattered light mirror coronagraph, has been in operation at the GermanArgentinean Solar Observatory in the Prof. Ulrico Cesco High Altitude Station of OAFA, El Leoncito, San Juan, Argentina since August, 1997. Several tests and calibration sequences were run during the first few months. In particular, tests were made for

- operational testing of the whole system,

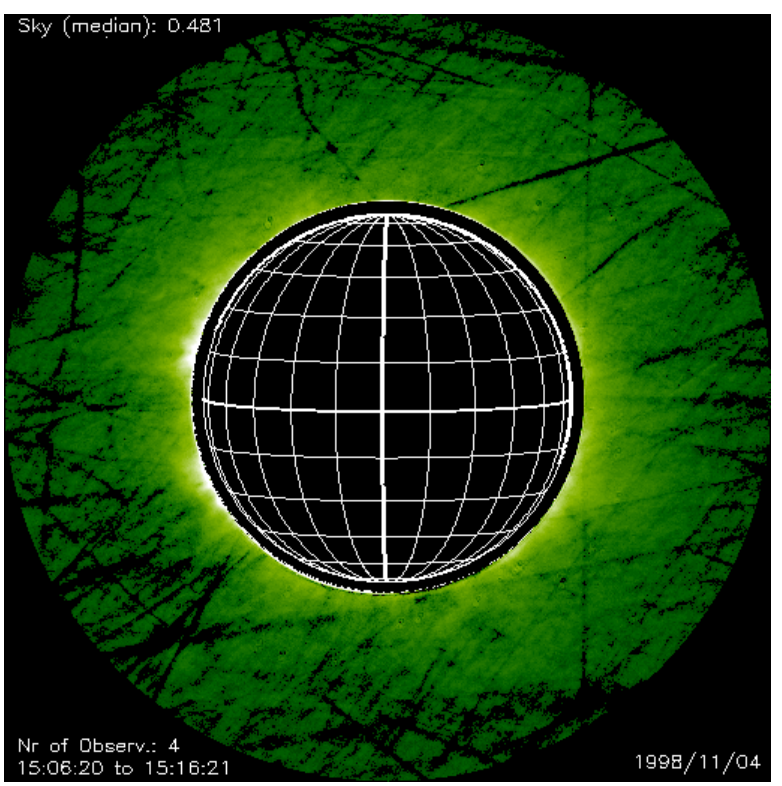

Figure 2.12: Effect of the "flying" seeds of the Aspen trees in the valley. The image shows the Fe XIV green line emission on November 4, 1998. It corresponds to the average of four raw "on-line" images taken in the time lapse between 15:06 UT and 15:16 UT. The black strips are seeds crossing the field of view of the instrument during the exposure time of the raw "off-line" images used to treat the "on-line" ones. For a detailed description of the reduction procedure of the raw images necessary to reveal the green line emission, the reader is referred to Chapter 3. The average sky quality (in $\mathrm{mV}$ ) as recorded by the sky tester is shown in the upper left corner of the image. 


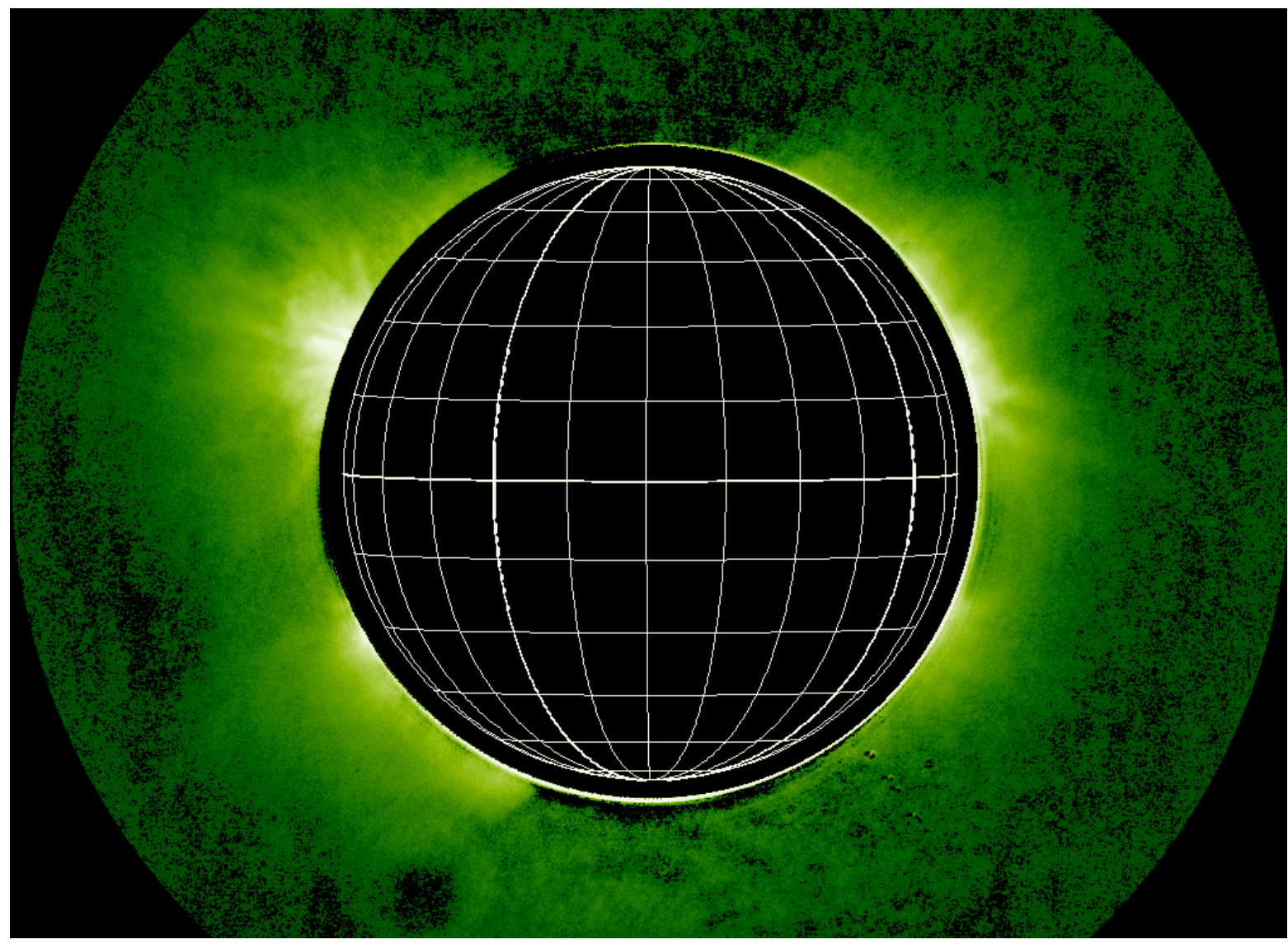

Figure 2.13: Fe XIV green coronal emission line on November 26, 1997 at 12:15 UT as observed by the MICA telescope (exposure time: $25 \mathrm{sec}$ ).

- development of algorithms for the stand-alone operation of the instrument, as e.g., for the automatic determination of the best exposure time, and for detection and analysis of both external (weather related) and internal (dome, door, filters, temperature, and camera related) factors,

- setting up of the working temperature of the filters, and

- determination of the optimal observing sequence.

Two and a half years of operation have shown the capabilities and reliability of the software developed.

In spite of being limited mainly by the brightness and temporal variability of the sky, MICA observations show a detailed view of the inner corona in both temporal and spatial scale (see, e.g., chapters 3 and 4, and Stenborg et al., 2000). Daily observations show that the MICA telescope is able to detect the relatively faint green coronal emission corresponding to the $\mathrm{Fe}^{13+}$ ions, i.e., the Fe XIV green line at $530.3 \mathrm{~nm}$, out to $1.6 \mathrm{R}_{\odot}$ with good temporal and spatial resolution (see, e.g., Figure 2.13). Moreover, the emission corona at two different temperature regimes can also be recorded at nearly the same time as exemplified in Figure 2.14. 


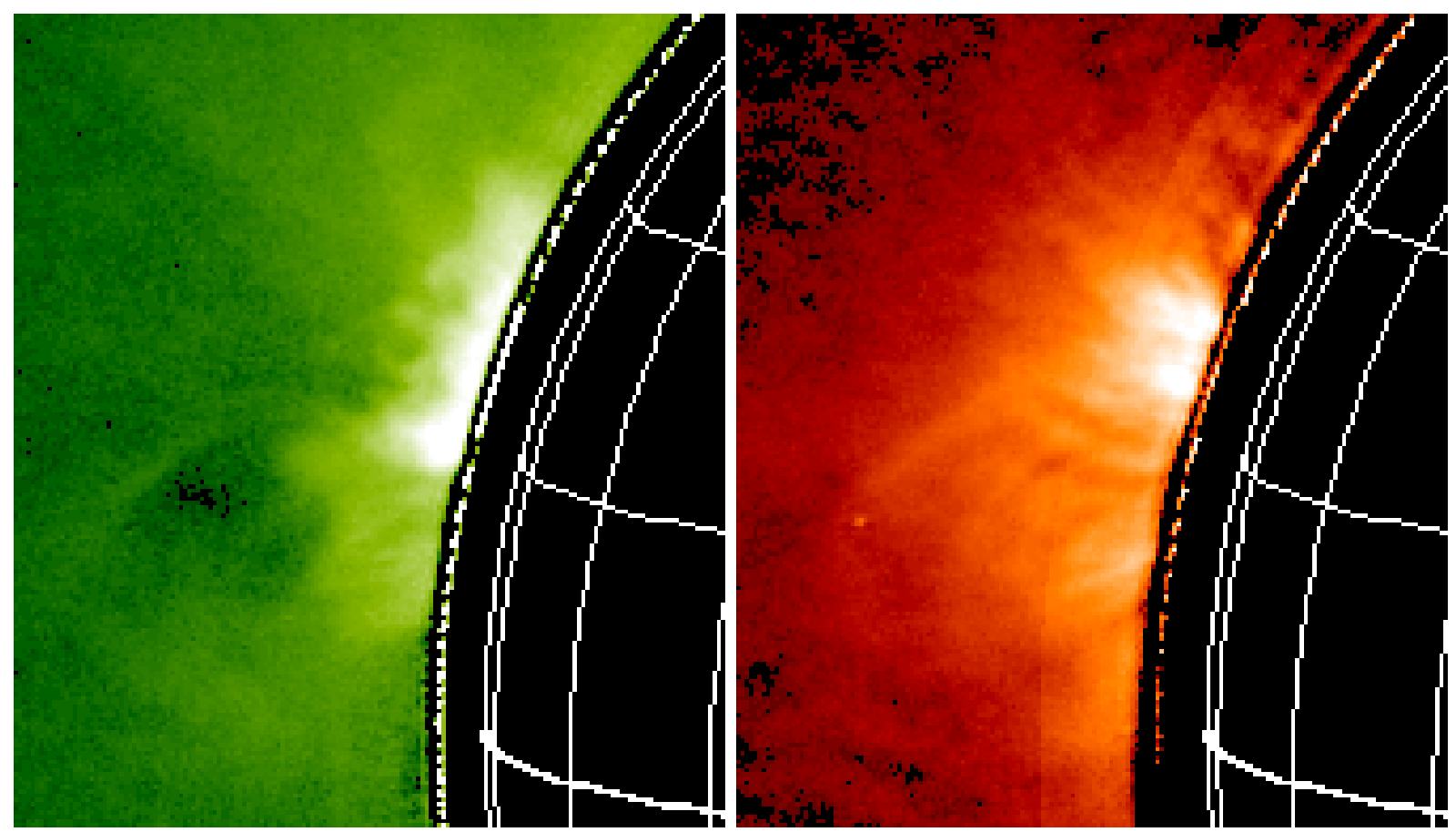

Figure 2.14: MICA observations of the same region at nearly the same time in two different wavelengths. Left: Fe XIV green emission line (530.3 nm) on August 23, 1997 at 15:52:44 UT (40 sec exposure). Right: Fe X red emission line (637.4 nm) on August 23, 1997 at 16:18:53 UT (40 sec exposure). 


\section{Chapter 3}

\section{The Calibration of the Observations}

The description of the reduction procedure specific for our problem will be the topic of the present Chapter, namely the detection of a faint coronal emission line with a ground-based coronagraph in presence of a strong and variable continuum background. After a brief introduction to the topic, the first section concentrates on a theoretical overview about the reduction procedure of the MICA images. Secondly, the corrections implemented to deal with instrumental constraints are presented. Then, the calibration of the data relative to the intensity of the continuum at the center of the solar disk is addressed. Further, the error introduced by the short-term sky brightness variability in the calibrated intensity is estimated. With all the elements at hand, the description of the especially developed real time reduction procedure, actually running at the site, is outlined. Finally, the validity of the hypotheses made and the reliability of the calibration procedure are tested by comparing MICA calibrated data with calibrated data of another ground-based instrument.

\subsection{Introduction}

Since the intensity of coronal emission line structures is much smaller than the scattered light background (both from the terrestrial atmosphere and the instrument itself), its detection is a real challenge. Deep understanding of the effects involved is necessary in order to derive credible calibrated coronal data.

Observations of coronal emission line structures taken with the help of either narrow band filters or Fabry Perot interferometers (e.g., with the LASCO-C1 instrument, Brueckner et al., 1995) are affected not only by scattered light but also have an additional contribution from the continuum (or 'white') corona ${ }^{1}$. The removal of the aforementioned contributions is necessary to reveal the coronal emission line structures. Basically, the procedure involves subtraction of continuum images (off-line images) from the on-line images. Such off-line images must be taken at a wavelength sufficiently far from line center, to avoid contamination by emission in the line itself. Furthermore, in order to reduce the effects of i) any rapid change of the continuum emission, i.e., rapid changes of the coronal electron density, ii) the solar rotation on the structures along the line of sight, and iii) the sky variability in case of ground-based coronagraphs, such as MICA, it is necessary to obtain the reference continuum images very close in time to the respective on-line images.

\subsection{A theoretical view}

The intrinsic flux distribution of the object under study cannot be measured directly because the light from the object is affected by many factors until it reaches the detector. During its propagation it can be absorbed or scattered

\footnotetext{
${ }^{1}$ The continuum corona is due to Thompson scattering of the photospheric light by electrons in the corona. See, e.g., Chapter 1 and references therein.
} 
in the Earth's atmosphere. Once in the telescope, its optical response and efficiency of the detecting device influence the measured flux as well. The measured flux is the convolution of the intrinsic flux and all of these factors. Thus, in wavelength space, the solar flux reaching the detector at time $t$ is represented by the count rate $C^{j, k}\left(\lambda_{i}, t\right)$ in the pixel $j, k$ of the CCD at the wavelength $\lambda_{i}$ as (in counts/sec):

$$
C^{j, k}\left(\lambda_{i}, t\right)=b^{j, k}+\int f_{i}(\lambda, t) \cdot \tau_{i}(\lambda, t) \cdot T_{i}^{j, k}\left(\lambda_{i}\right) \cdot g \cdot D_{i}^{j, k}\left(\lambda_{i}\right) \cdot \Omega \cdot S d \lambda
$$

where

- $f_{i}(\lambda, t)$ represents the intrinsic flux of the source with units of photons $\mathrm{sec}^{-1} \mathrm{~cm}^{-2} \mathrm{sr}^{-1}$,

- $\tau_{i}(\lambda, t)$ depicts the wavelength dependence of the transmission coefficient of the Earth's atmosphere (Appendix B.2),

- $T_{i}^{j, k}\left(\lambda_{i}, t\right)$ denotes the combined optical response of the filters and mirrors (i.e., the optical response of the telescope),

- $g$ represents the conversion factor (in $D N / e^{-}$),

- $D_{i}^{j, k}\left(\lambda_{i}\right)$ symbolizes the quantum efficiency of each pixel (in $e^{-} /$photon),

- $S$ represents the effective aperture of the telescope (in $\mathrm{cm}^{2}$ ),

- $\Omega$ is the solid angle subtended by one pixel (in $s r$ ), and

- $b^{j, k}$ is the signal in counts/sec delivered by the detector in the absence of any incident signal (i.e., both the bias and residual charge after cooling of the detector device) $)^{2}$.

The integration range mainly depends upon the filter transmission. During the rest of the Chapter, the supraindex $j, k$ denoting the pixel position is suppressed for simplicity. It will be explicitly used only when strictly necessary to avoid confusion.

The problem can be simplified by denoting the contributions from the optics and detector simply as the total response function of the instrument $Q(\lambda)$, i.e., $Q(\lambda)=T_{i}^{j, k}\left(\lambda_{i}\right) \cdot g \cdot D_{i}^{j, k}\left(\lambda_{i}\right)$. The factor $Q(\lambda)$ is usually known as the total system quantum efficiency. In that case:

$$
C\left(\lambda_{i}, t\right)-b=\Omega \cdot S \cdot \int f_{i}(\lambda, t) \cdot \tau_{i}(\lambda, t) \cdot Q_{i}(\lambda) d \lambda
$$

\section{The signals detected by MICA}

Let $C\left(\lambda_{i}, t\right)$ denote the number of counts/sec (i.e., the count rate) measured in each pixel of the CCD, already corrected by bias and dark (the term $b$ in Eq. 3.1). We now address the reduction process of the MICA images in terms of (3.1). Thus, let $C\left(\lambda_{l}\right)$ be the count rate with the filter corresponding to the emission line, and $C\left(\lambda_{c}\right)$ with

\footnotetext{
${ }^{2}$ For a detailed description of the parameters related to the detecting device, i.e., $g, D_{i}^{j, k}\left(\lambda_{i}\right)$, and $b^{j, k}$, the reader is referred to Section 2.4.1.
} 
the filter corresponding to the nearby continuum. $C\left(\lambda_{l}\right)$ has the contribution not only from the line but also from the continuum and scattered light in both the instrument and the terrestrial atmosphere (for the following discussion I will refer it globally as the scattered light contribution). On the other hand, $C\left(\lambda_{c}\right)$ has only the contribution from the continuum and scattered light. With all this in mind, Equation (3.1) is rewritten for the line and continuum images as

$$
C\left(\lambda_{l}, t\right)=\Omega \cdot S \cdot \int\left[\left(A_{l}(\lambda, t) \cdot \tau_{l}(\lambda, t)\right)+H_{l}^{s}(\lambda, t)\right] \cdot Q_{l}(\lambda) d \lambda
$$

where

$$
A_{l}(\lambda, t)=f_{l}(\lambda, t)+f_{c}(\lambda, t)+H_{l}^{i}(\lambda, t)
$$

and

$$
C\left(\lambda_{c}, t+\delta t\right)=\Omega \cdot S \cdot \int\left[\left(A_{c}(\lambda, t+\delta t) \cdot \tau_{c}(\lambda, t+\delta t)\right)+H_{c}^{s}(\lambda, t+\delta t)\right] \cdot Q_{c}(\lambda) d \lambda
$$

where

$$
A_{c}(\lambda, t+\delta t)=f_{c}(\lambda, t+\delta t)+H_{c}^{i}(\lambda, t+\delta t)
$$

$H_{l}$ and $H_{c}$ denote the scattered light contribution at the respective wavelengths, the supraindexes $i$ and $s$ indicating here "in the instrument" and "in the terrestrial atmosphere", respectively. $H^{i}$ is mainly due to scattering of the solar disk radiation in the mirror $M_{1}$ (Figure 2.2), which is subjected to the full un-occulted view of the Sun.

\section{Flatfielding}

As mentioned above, the quantum efficiency factor $D_{i}^{j, k}\left(\lambda_{i}\right)$ is different for every pixel. Therefore, the individual "gain" factors, i.e., $g \cdot D_{i}^{j, k}\left(\lambda_{i}\right)$, have to be determined. This is done by exposing the whole CCD to a calibrated diffuse light source, i.e., the so-called "flat fields". In case of the MICA instrument, they are normal images exposed to the full Sun taken with the entrance door closed. The MICA door lid contains a quartz diffusor. When the door is closed, attenuated, diffuse light from the solar disk illuminates $\mathrm{A}_{0}$ (Figure 2.2) and is transmitted to the focal plane. This radiation provides flat field illumination with solar spectral content. Thus, if we use $F$ to denote the flat field images (corrected by bias and dark), and $E_{0}$ to represent the intrinsic flux of the Sun, we have

$$
F\left(\lambda_{l}, t^{\prime}\right)=\Omega \cdot S \cdot \int\left[\left(\left(E_{0}\left(\lambda, t^{\prime}\right)+H_{l}^{i}\left(\lambda, t^{\prime}\right)\right) \cdot \tau_{l}\left(\lambda, t^{\prime}\right)\right)+H_{l}^{s}\left(\lambda, t^{\prime}\right)\right] \cdot Q_{l}(\lambda) d \lambda
$$

and

$$
F\left(\lambda_{c}, t^{\prime \prime}\right)=\Omega \cdot S \cdot \int\left[\left(\left(E_{0}\left(\lambda, t^{\prime \prime}\right)+H_{c}^{i}\left(\lambda, t^{\prime \prime}\right)\right) \cdot \tau_{c}\left(\lambda, t^{\prime \prime}\right)\right)+H_{c}^{s}\left(\lambda, t^{\prime \prime}\right)\right] \cdot Q_{c}(\lambda) d \lambda
$$

where $t^{\prime}$ and $t^{\prime \prime}$ are used here to explicitly show the fact that they are taken at different times from one another and from the respective open door images. Note that for the flats we considered no emission signal from the corona because, unlike the open door images in which the disk of the Sun is occulted, the flats are pure photospheric light passing through the diffusor, which effectively overpowers the relatively weak emission signal from the corona. Moreover, the scattered light contribution, both from the sky and inside the instrument, is negligible compared to the diffused sunlight, i.e, 


$$
\begin{gathered}
\frac{H_{l}^{s}(\lambda, t)}{\left(A_{l}(\lambda, t) \cdot \tau_{l}(\lambda, t)\right)} \gg \frac{H_{l}^{s}\left(\lambda, t^{\prime}\right)}{\left(\left(E_{0}\left(\lambda, t^{\prime}\right)+H_{l}^{i}\left(\lambda, t^{\prime}\right)\right) \cdot \tau_{l}\left(\lambda, t^{\prime}\right)\right)}, \\
\frac{H_{c}^{s}(\lambda, t+\delta t)}{\left(A_{c}(\lambda, t+\delta t) \cdot \tau_{c}(\lambda, t+\delta t)\right)} \gg \frac{H_{c}^{s}\left(\lambda, t^{\prime \prime}\right)}{\left(\left(E_{0}\left(\lambda, t^{\prime \prime}\right)+H_{c}^{i}\left(\lambda, t^{\prime \prime}\right)\right) \cdot \tau_{c}\left(\lambda, t^{\prime \prime}\right)\right)}, \\
H_{l}^{i}\left(\lambda, t^{\prime}\right) \ll E_{0}\left(\lambda, t^{\prime}\right),
\end{gathered}
$$

and

$$
H_{c}^{i}\left(\lambda, t^{\prime \prime}\right) \ll E_{0}\left(\lambda, t^{\prime}\right)
$$

Given the characteristics of the flat field images, it is convenient to define the flatfielding procedure simply as $C\left(\lambda_{l}, t\right) / F\left(\lambda_{l}, t^{\prime}\right)$ and $C\left(\lambda_{c}, t+\delta t\right) / F\left(\lambda_{c}, t^{\prime \prime}\right)$. This allows to get rid of the attenuation effect of the atmosphere in the signal ${ }^{3}$, provided the flats are taken very close in time to the respective open door images (i.e., $t^{\prime} \rightarrow t$ and $\left.t^{\prime \prime} \rightarrow t+\delta t\right)^{4}$. Moreover, since the optics and detector are the same in both kind of exposures, the effective aperture of the telescope $S$ and pixel size $\Omega$ also cancel out. Therefore, dividing (3.2) by (3.4) and (3.3) by (3.5) considering previously the approximations mentioned above, and after some algebra we obtain

$$
C^{f}\left(\lambda_{l}, t\right)=\Upsilon_{l} \cdot \frac{\int_{\lambda} A_{l}(\lambda, t) \cdot Q_{l}(\lambda, t) \cdot d \lambda}{\int_{\lambda} E_{0}\left(\lambda, t^{\prime}\right) \cdot Q_{l}\left(\lambda, t^{\prime}\right) \cdot d \lambda}+\Upsilon_{l} \cdot \tau_{l}^{-1}(t) \cdot \frac{\int_{\lambda} H_{l}^{s}(\lambda, t) \cdot Q_{l}(\lambda, t) \cdot d \lambda}{\int_{\lambda} E_{0}\left(\lambda, t^{\prime}\right) \cdot Q_{l}\left(\lambda, t^{\prime}\right) \cdot d \lambda}
$$

and

$$
\begin{array}{r}
C^{f}\left(\lambda_{c}, t+\delta t\right)=\Upsilon_{c} \cdot \frac{\int_{\lambda} A_{c}(\lambda, t+\delta t) \cdot Q_{c}(\lambda, t+\delta t) \cdot d \lambda}{\int_{\lambda} E_{0}\left(\lambda, t^{\prime \prime}\right) \cdot Q_{c}\left(\lambda, t^{\prime \prime}\right) \cdot d \lambda}+ \\
\quad+\quad \Upsilon_{c} \cdot \tau_{c}^{-1}(t+\delta t) \cdot \frac{\int_{\lambda} H_{c}^{s}(\lambda, t+\delta t) \cdot Q_{c}(\lambda, t+\delta t) \cdot d \lambda}{\int_{\lambda} E_{0}\left(\lambda, t^{\prime \prime}\right) \cdot Q_{c}\left(\lambda, t^{\prime \prime}\right) \cdot d \lambda}
\end{array}
$$

where the supraindex $f$ is used here to indicate flatfielded images. The factors $\Upsilon_{l} \rightarrow 1$ and $\Upsilon_{c} \rightarrow 1$ denote the error introduced by having $t^{\prime} \rightarrow t$ and $t^{\prime \prime} \rightarrow t+\delta t$ and not $t^{\prime}=t$ and $t^{\prime \prime}=t+\delta t$ (see Section 3.3.5).

The value each pixel reports after such a division is representative of the coronal signal (plus potential contaminants) in units of the intensity of the solar disk at that wavelength as measured by the flat fields. In sections 3.3.2 and 3.3.3 is shown how this number is then converted to parts per million ( $p p m)$ of the intensity of the solar disk at that wavelength.

\section{Comment on the sky brightness}

We now rewrite the term corresponding to the scattered light in the terrestrial atmosphere $\left(H^{s}\right)$ in (3.6) and (3.7). According to Volz (1954), the sky brightness around the solar disk can be represented by

$$
H_{\lambda}^{s}(t)=E_{0}(t) \cdot \Gamma_{\lambda}(\varphi, t) \cdot X(z, t) \cdot \tau_{\lambda}(t)
$$

\footnotetext{
${ }^{3}$ The transmission coefficient of the Earth's atmosphere can be considered constant within the integration range defined by the filter characteristics, i.e., $\tau_{i}(\lambda, t) \approx \tau_{i}(t)$

${ }^{4}$ A more detailed study of the effect of the transmittance of the Earth's atmosphere $\tau$ on the calibration, due to the finite time lapse between open and closed door images is carried out in sections 3.3.5 and 3.3.6.
} 
where $X(z, t)$ represents the air mass at the observation site $z$ at time $t$ (Appendix B.1), and $\Gamma_{\lambda}(\varphi)$ the scattering function at the wavelength $\lambda$ under the scattering angle $\varphi$.

By replacing (3.8) in (3.6) and (3.7) we obtain the following functional form for the flatfielded images:

$$
C^{f}\left(\lambda_{l}, t\right)=\Upsilon_{l} \cdot L_{1}\left(\lambda_{l}, t\right)+\Upsilon_{l} \cdot L_{2}\left(\Gamma_{\lambda_{l}}(\varphi), \lambda_{l}, t\right) \cdot X(z, t)
$$

and

$$
C^{f}\left(\lambda_{c}, t+\delta t\right)=\Upsilon_{c} \cdot C_{1}\left(\lambda_{c}, t+\delta t\right)+\Upsilon_{c} \cdot C_{2}\left(\Gamma_{\lambda_{c}}(\varphi), \lambda_{c}, t+\delta t\right) \cdot X(z, t+\delta t)
$$

In this way, the sky light contribution is represented in the flatfielded images exclusively by the $L_{2}$ and $C_{2}$ terms. This issue is addressed in deeper detail in Section 3.3.5.

\section{Continuum and scattered light removal}

Let us address now the next step in the calibration procedure, that is the removal of the continuum and scattered light. The key point for that task is to take the continuum images as close in time as possible to the emission line images (i.e, $\delta t \rightarrow 0$ ), in order to mainly reduce the effects of the sky variability, which are due to changes in $\Gamma_{\lambda}(\varphi)$ (see equations 3.9 and 3.10). Furthermore, the effects of solar rotation along the line of sight are also avoided, as well as transient changes in the continuum emission occurring in time lapses $<\delta t$.

In the hypothetical case $\delta t=0$ (and $t=t^{\prime}=t^{\prime \prime}$, i.e., $\Upsilon_{l}=1$ and $\Upsilon_{c}=1$ ) one expects to have the same continuum and instrumental scattered light contribution for both on- and off-line images. In this case, the sky variability is also meaningless, although $L_{2}$ (in Eq. 3.9) is slightly different from $C_{2}$ (in Eq. 3.10) due to the wavelength dependence of $\Gamma_{\lambda}(\varphi)$. Then, by accepting that constraint as a limit $\delta R$ to the possible accuracy of the calibration procedure one could use

$$
E\left(\lambda_{l}, t\right)+\delta R=C^{f}\left(\lambda_{l}, t\right)-C^{f}\left(\lambda_{c}, t\right)
$$

to obtain the intensity of the coronal emission line $E\left(\lambda_{l}, t\right)$ at time $t$. However, in the real case it is impossible to take both the open (line and continuum) and closed door (flat field) images such that $t=t+\delta t=t^{\prime}=t^{\prime \prime}$. In that case, the effects of the sky variability are added to the residual sky light $\delta R$ arising from the dependence of the scattering function $\Gamma_{\lambda}(\varphi)$ with wavelength.

Therefore, the continuum measurements must be scaled to the intensity of the on-line measurements by multiplying them by a scaling factor $\beta$, defined as

$$
\beta=\frac{<\left[\Upsilon_{l} \cdot C^{f}\left(\lambda_{l}, t\right)\right]_{\infty}>}{<\left[\Upsilon_{c} \cdot C^{f}\left(\lambda_{c}, t+\delta t\right)\right]_{\infty}>}
$$

in such a way that:

$$
E\left(\lambda_{l}, t\right)=\Upsilon_{l} \cdot C^{f}\left(\lambda_{l}, t\right)-\beta \cdot \Upsilon_{c} \cdot C^{f}\left(\lambda_{c}, t+\delta t\right)
$$

The subindex $\infty$ in (3.11) indicates a position in the image where the emission line signal is negligible. The practical implementation of (3.11) is outlined in Section 3.3.4. 


\section{Line profile correction}

Up to now, only the photometric correction with respect to the continuum was applied, under the following assumptions: i) that the flat fields must be taken close in time to the respective images to reduce to a minimum the effect of changes in the transmittance of the Earth's atmosphere, and ii) that the continua must also be taken very close in time to the on-line images to remove properly the scattered light and continuum. The last step is to take into account the influence of the telescope transmission profile $Q_{l}(\lambda, T)$ over the intrinsic line profile. The argument $T$ is written to denote explicitly the temperature dependence of the overall response of the telescope, strongly dependent upon the filter transmission profile.

Let $e_{l}(\lambda, t)$ be the intrinsic flux of the coronal emission line. Hence Equation (3.12) can be thought of as

$$
E\left(\lambda_{l}, t\right)=\frac{\int_{\lambda} e_{l}(\lambda, t) \cdot Q_{l}(\lambda, T) \cdot d \lambda}{\int_{\lambda} Q_{l}(\lambda, T) \cdot d \lambda}
$$

Let $\varphi(\lambda, t)$ be the intrinsic profile of the coronal emission line such that

$$
e(\lambda, t)=\widehat{E}(t) \cdot \varphi(\lambda, t)
$$

with $\widehat{E}(t)=$ constant in terms of $\lambda$. Then (3.13) becomes

$$
E\left(\lambda_{l}, t\right)=\frac{\widehat{E}(t) \cdot \int_{\lambda} \varphi(\lambda, t) \cdot Q(\lambda, T) \cdot d \lambda}{\int_{\lambda} Q(\lambda, T) \cdot d \lambda}
$$

The total line emission $E_{0}(t)$ at time $t$ is

$$
E_{0}(t)=\widehat{E}(t) \cdot \int_{\lambda} \varphi(\lambda, t) d \lambda \Rightarrow \widehat{E}(t)=\frac{E_{0}(t)}{\int_{\lambda} \varphi(\lambda, t) d \lambda}
$$

Finally, replacing (3.15) in (3.13) and rearranging terms

If we define

$$
E_{0}(t)=E\left(\lambda_{l}, t\right) \cdot \frac{\left[\int Q_{l}(\lambda, T) d \lambda\right]\left[\int \varphi(\lambda, t) d \lambda\right]}{\int \varphi(\lambda, t) \cdot Q_{l}(\lambda, T) d \lambda}
$$

$$
\frac{\left[\int Q_{l}(\lambda, T) d \lambda\right]\left[\int \varphi(\lambda, t) d \lambda\right]}{\int \varphi(\lambda, t) \cdot Q_{l}(\lambda, T) d \lambda} \doteq f_{k}
$$

(3.16) becomes

$$
E_{0}(t)=E\left(\lambda_{l}, t\right) \cdot f_{k}
$$

The so-called normalization factor $f_{k}$ depends strongly upon temperature through $Q(T)$. The hypotheses necessary for the practical calculation of this factor as well as its estimate will be outlined in detail in Section 3.3.7.

\subsubsection{Summary}

A theoretical view of the reduction procedure of the MICA images has been given. Briefly, we summarize here the main points to be taken into account. In the ideal case, the procedure outlined can be schematized as shown in Figure 3.1.

However, it has been shown that the validity of the block diagram shown in Figure 3.1 is limited by several instrumental constraints. The main ones can be summarized as follows: 
- Impossibility of obtaining on- and off-line images simultaneously. The simultaneity of the observations is required to avoid

- sky intensity changes,

- solar rotation effects, and

- changes of the continuum intensity between on- and off-line images due to fast transient phenomena.

- Impossibility of obtaining open (on- and off-line) and the respective closed (flat field) door images simultaneously. Since the flat fields are used for calibration purposes, i.e., to express the on- and off-line images in terms of the intensity at the center of the disk, the atmospheric extinction plays a fundamental role. Therefore, simultaneous observations allow getting rid of the effects of the transmittance of the Earth's atmosphere due to i) different air masses and ii) the variability of the extinction coefficient.

- Constancy of the total response function of the instrument, which has a strong dependence on temperature.

Besides the instrumental constraints, a physical constraint contributes to the error budget, i.e, the intensity of the continuum radiation emitted by the Sun is not the same at the line and continuum wavelengths. This difference

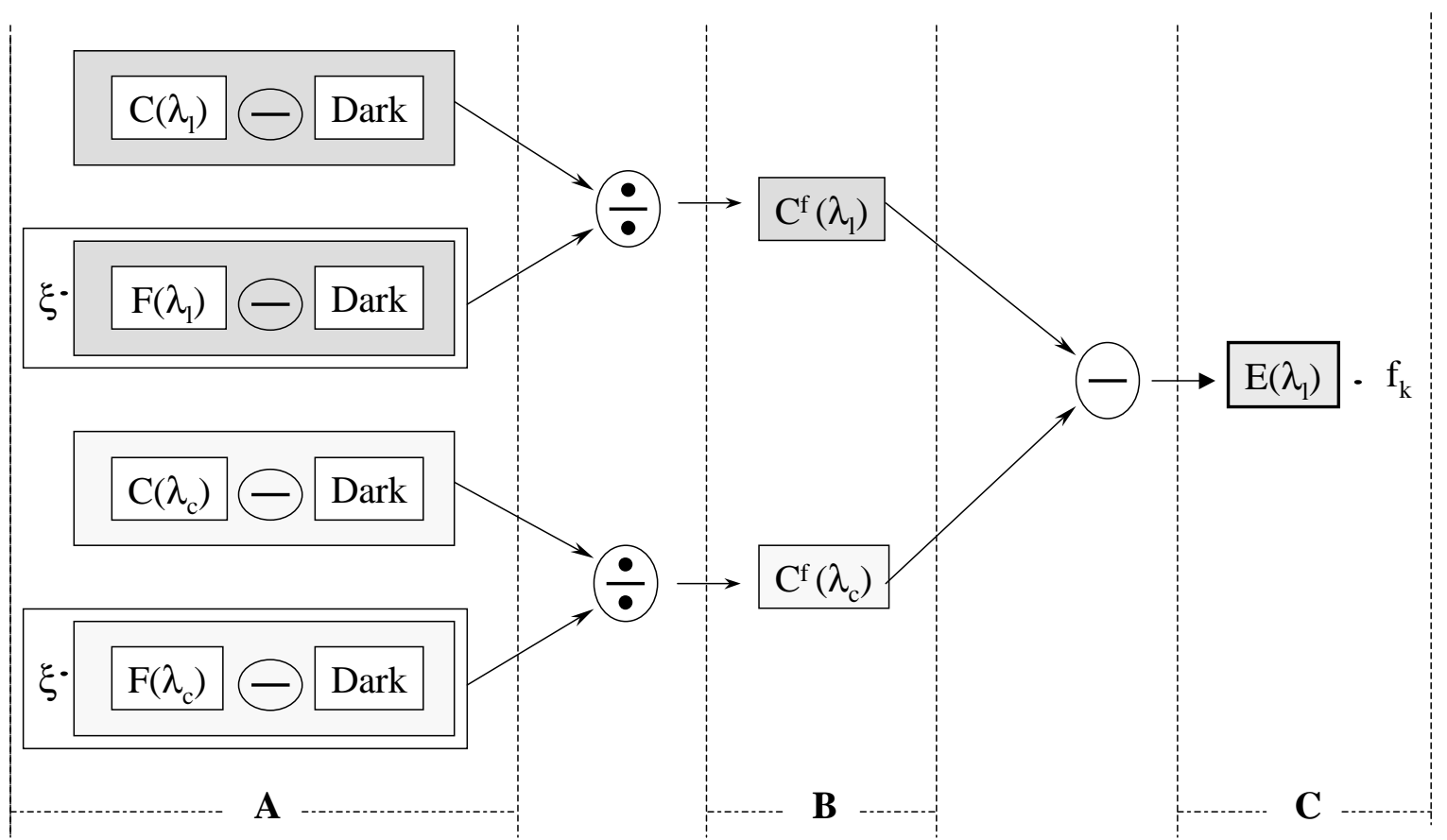

Figure 3.1: Block diagram of the reduction technique that would be used on MICA images in the ideal case, i.e., both on-, and off-line images and the respective flats to be taken at the same time, and the spectral distribution of the Sun's continuum radiation assumed to be wavelength independent. The so-called calibration factor $\xi$ is used to express the flat field images in terms of a physical unit, i.e., the intensity at the center of the solar disk (see sections 3.3.2 and 3.3.3). The notation used here is the same as that used in Section 3.2. 
must be accounted for when expressing the on- and off-line images in terms of the intensity of the continuum at the center of the disk (see Section 3.3.3). Several factors were introduced in the equations used in order to account for these constraints:

- $\Upsilon_{l}$ and $\Upsilon_{c}$ : They denote the error introduced by taking the flat fields at different time of the respective onand off-lines images. They arise from the different air masses involved and the variability of the atmospheric extinction coefficient.

- $\beta$ : This factor is computed to correct for the residual sky light after subtracting the off-line images from on-line ones (both of them expressed in terms of the continuum intensity at the center of the solar disk). Its value accounts therefore for the sky intensity variation (due to the variability of the extinction coefficient and different air masses between on- and off- line images). The departure of the ideal case measured by $\Upsilon_{c}$ and $\Upsilon_{l}$ is also accounted for with the correction factor $\beta$.

- $f_{k}$ : This normalization factor corrects for the telescope transmission profile.

The block diagram of the reduction procedure practically used is shown in Figure 3.2. In the following section we outline the different steps performed for the practical implementation of the reduction and calibration of MICA images. Briefly, they include

- development of the basic observation routine (Section 3.3.1),

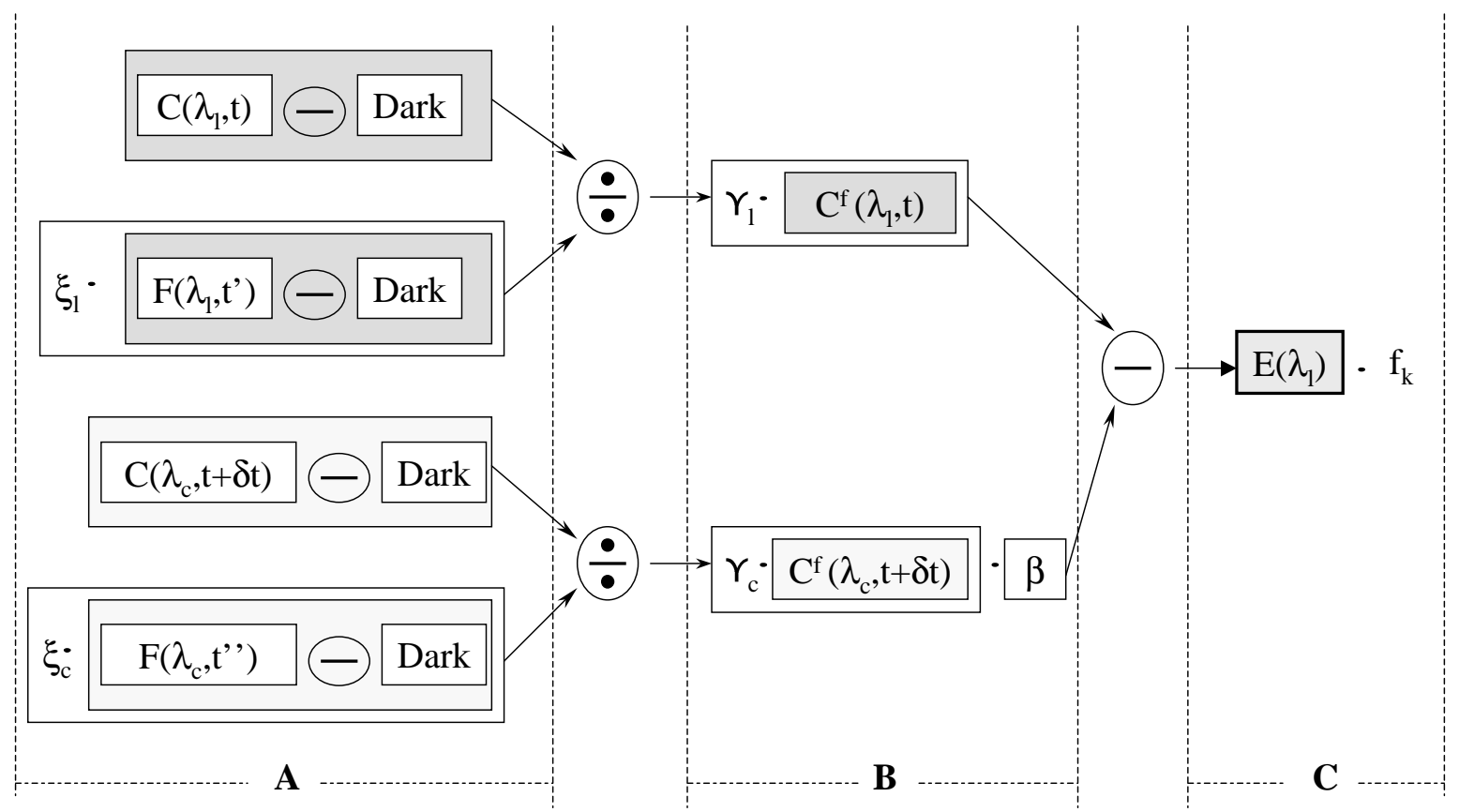

Figure 3.2: Block diagram of the reduction technique applied to MICA images. Note that the time at which the corresponding images are taken is explicitly denoted. Moreover, the wavelength dependence of the so-called calibration factor $\xi$ (sections 3.3.2 and 3.3.3) is also denoted with the subindexes $l$ (line) and $c$ (continuum). The notation used here is the same as that used in Section 3.2. For details see text. 
- determination of the calibration factor for the flat field images (Section 3.3.2),

- practical implementation of the conversion of the on- and off-line images to relative units and continuum removal (Section 3.3.3),

- determination of the scaling factor $\beta$ (Section 3.3.4), and

- determination of the normalization factor $f_{k}$ for each observation (Section 3.3.7).

Moreover, since the instantaneous determination of the factors $\Upsilon_{l}$ and $\Upsilon_{c}$ is not possible, we analyze qualitatively how the calibration is affected by the varying transmittance of the Earth's atmosphere between observations (Section 3.3.5). Finally, we show how the scaling factor $\beta$, which is used to correct variations in the sky brightness can be used to estimate the accuracy of the calibration procedure (Section 3.3.6).

\subsection{Practical implementation of the reduction and calibration procedure of MICA images}

\subsubsection{On the development of the basic observation sequence}

The variability of the sky brightness, i.e., rapid changes in the transmission of the Earth's atmosphere $\tau_{i}$, will not influence the emission line signal obtained if the on-line images are taken sufficiently close in time to the continuum ones that are used to subtract the extra contributions (i.e., sky, instrumental straylight, and background continuum). Furthermore, the flats are required for calibration purposes as they reflect the intensity at the center of the solar disk at the time of the observation. For this reason, they also have to be taken as close as possible in time to the respective images to avoid the effects produced by the sky variability.

Any observation sequence must account for all these constraints. Let $L$ denote an on-line exposure, $C$ a continuum exposure and $F_{L}$ and $F_{c}$ the respective flats. Then, the following sequence definition satisfies the requirements:

$$
\underbrace{L \cdots L}_{n \text { times }} C L C F_{L} F_{C} \underbrace{L \cdots L}_{n \text { times }} C L C
$$

where the optimum value for $n$ is fixed to 3 according to the experience gained at the site during the first two years of operation. Note that continuum images are sandwiched between on-line images. This fact allows to check the constancy of the sky. This basic unit, so-called sequence, is the basal stone on which the default observation routine was built.

\subsubsection{On the determination of the calibration factor $\xi$}

The number of counts/sec collected by the detector at the selected wavelength has to be expressed in terms of a physical unit, e.g., the intensity of the continuum at that specific wavelength at the center of the solar disk, i.e., $B_{\odot}\left(\lambda_{i}\right)$. To do so, a comparison with the counts/sec the detector would measure with direct observation at 


\begin{tabular}{lccc}
\hline \hline Filter & $\lambda($ in $n m)$ & $\xi$ & $\sigma$ \\
\hline Green line & 530.3 & 136000 & 5000 \\
Green continuum & 526.0 & 143000 & 5000 \\
Red line & 637.4 & 161000 & 3000 \\
Red Continuum & 634.0 & 108000 & 3000 \\
\hline \hline
\end{tabular}

Table 3.1: Calibration factor $\xi_{i}$ at different wavelengths.

that wavelength of the center of the solar disk under similar air mass must be performed. For that purpose a calibrated neutral density (ND) filter has to be applied in order to compensate for the extreme brightness difference between the faint corona and the solar disk. Direct exposition of the CCD to the Sun would result in permanent damage of the detector. In practice, it is not needed to observe the solar disk after each observation, since there exists a relationship between the flat field images and such observations of the solar disk, which was found to be constant within the limits of accuracy actually involved. The conversion factor obtained is referred hereafter as the calibration factor $\xi$. For this reason, flat fields must be taken at a relatively high cadence in order to account for a reliable estimation of the intensity at the center of the solar disk under the relative same conditions existent at the time of the normal observations.

Briefly, the procedure to determine $\xi$ consists of observing the solar disk with the filter $i$ plus a ND filter of known transparency, i.e., $\overline{\operatorname{Sun}}\left(\lambda_{i}\right)$, and the corresponding flat field with the same filter but without the ND one, i.e., $\overline{\text { Flat }}\left(\lambda_{i}\right)$. The former observation is performed by placing the solar image into one corner of the field of view. After bias and flatfielding correction, and exposure time normalization, a second degree surface is fitted to the Sun image. The maximum of such a surface is taken as the calibration factor $\xi_{i}$ ( $i$ indicates the filter used). In other words:

$$
\begin{aligned}
& \text { 1. } \bar{S}\left(\lambda_{i}\right)=\frac{\overline{\operatorname{Sun}}\left(\lambda_{i}\right) \cdot t_{\text {sun }}^{-1}}{\overline{\text { Flat }\left(\lambda_{i}\right) \cdot t_{\text {flat }}^{-1}}} \\
& \text { 2. } I_{\max }\left(\lambda_{i}\right)=\max \left(S\left(\lambda_{i}\right)\right) \\
& \text { 3. } \xi\left(\lambda_{i}\right)=I_{\text {max }} \cdot 10^{-N D\left(\lambda_{i}\right)}
\end{aligned}
$$

where $t_{\text {sun }}$ and $t_{\text {flat }}$ respectively indicate the corresponding exposure time and it is tacitly assumed that the images are bias and dark corrected prior to any operation. $N D\left(\lambda_{i}\right)$ is the attenuation factor at the wavelength $\lambda_{i}$ of the neutral density filter used.

Since both images are taken very close in time, we consider that the transmittance of the Earth's atmosphere does not change between the observations (i.e., the hypotheses discussed in the previous section in regard to the flatfielding procedure are considered). Moreover, the procedure is repeated for the four corners along the day to test the dependence of the results across the field of view and with different air masses (i.e., at different times of the day). Within the experimental accuracy, no discrepancies were found, this fact confirming the hypotheses made. A detail of the calibration factors $\xi_{i}$ obtained for the different filters used is shown in Table 3.1. 
In this way, the number of counts/sec collected by any flat field multiplied by the factor $\xi_{i}$ (wavelength dependent) gives a good estimate of the number of counts/sec the CCD would measure in a direct observation of the Sun at that wavelength. The reliability of this factor is implicitly tested in Section 3.5 by comparing MICA calibrated green line images with similar data of another calibrated instrument. The flat field images are thus representative of the intensity at the center of the solar disk $B_{\odot}$ under the atmospheric conditions existent at the time of the measurement. In other words, by using $f^{j, k}$ to denote the numbers of counts recorded in the pixel $(j, k)$ in a $t$ sec flat field exposure, we can write

$$
\frac{\xi_{i} \cdot f^{j, k}}{t} \text { counts sec }^{-1}=B_{\odot}
$$

Therefore

$$
1 \text { count } \sec ^{-1}=\frac{t}{\xi_{i} \cdot f^{j, k}} B_{\odot}
$$

\section{Discussion}

The most important assumption made for the determination of $\xi\left(\lambda_{i}\right)$ is that the images taken for such a determination, i.e., $\overline{\operatorname{Sun}}\left(\lambda_{i}\right)$ and $\overline{\text { Flat }}\left(\lambda_{i}\right)$, are obtained very close in time, in order to have similar air masses and extinction coefficients for both exposures. In this way, the resulting ratio number $\xi\left(\lambda_{i}\right)$ becomes independent of the attenuation effects suffered by the radiation in the terrestrial atmosphere, provided the scattered light contribution (terms containing $H_{l}^{s}$ and $H_{c}^{s}$ in equations 3.6 and 3.7) in the flat field images is negligible as compared to the signal produced by the incoming sunlight.

\subsubsection{On the conversion of the on- and off-line images to relative units and continuum removal}

Once the calibration factor $\xi\left(\lambda_{i}\right)$ is obtained, we can express the on- and off-line images in parts per million ( $\left.p p m\right)$ with respect to the intensity at the center of the solar disk at that specific wavelength $B_{\odot}\left(\lambda_{i}\right)$. Making use of (3.18), the conversion is simply expressed by

$$
I\left(\lambda_{i}\right)_{\left[B_{\odot}\left(\lambda_{i}\right)\right]}=\Upsilon_{i} \cdot \frac{C\left(\lambda_{i}\right) \cdot t_{C\left(\lambda_{i}\right)}^{-1}}{\left(F\left(\lambda_{i}\right) \cdot t_{F\left(\lambda_{i}\right)}^{-1}\right) \cdot \xi\left(\lambda_{i}\right)}
$$

As aforementioned, $C\left(\lambda_{i}\right)$ indicates either the on- or off-line image, $F\left(\lambda_{i}\right)$ the respective flat field, $t_{C\left(\lambda_{i}\right)}$ and $t_{F\left(\lambda_{i}\right)}$ the corresponding exposure times, $\Upsilon_{i} \rightarrow 1$ the factor that shows the departure of the ideal case by taking into account the variability of the transmission of the Earth's atmosphere $\tau_{i}$ between the image and the respective flat field, and it is assumed that the images are already corrected by bias and dark. The subindex $\left[B_{\odot}\left(\lambda_{i}\right)\right]$ was used in the left side of (3.19) to explicitly denote the units of the resulting flatfielded image.

At wavelengths redward of about $500 \mathrm{~nm}$, the continuum intensity emitted by the photosphere lies close to the curve calculated for a $5760 \mathrm{~K}$ black body. (see Appendix A). That means, rigourously we cannot compare the intensity expressed in ppm for the on-line image (at $530.3 \mathrm{~nm}$ ) with that for the continuum image (at $526.0 \mathrm{~nm}$ ). In 
the ideal case of a flat continuum spectrum, the use of ppm becomes independent of the wavelength at which the observation is performed. Only in this hypothetical case, $\left[B_{\odot}\left(\lambda_{l}\right)\right]$ and $\left[B_{\odot}\left(\lambda_{c}\right)\right]$ represent the same kind of units $\left[B_{\odot}\right]$. Therefore, one could write (3.12) as

$$
E\left(\lambda_{l}\right)_{\left[B_{\odot}\right]}=I\left(\lambda_{l}\right)_{\left[B_{\odot}\right]}-\beta \cdot I\left(\lambda_{c}\right)_{\left[B_{\odot}\right]}
$$

with the scaling factor $\beta$ given by (3.11). The letter $E$ is used here instead of $I$ to indicate the final processed image with the extra contributions removed. However, for the real case of the spectral distribution of the Sun's continuum radiation, the following relation can be considered:

$$
\left[B_{\odot}\left(\lambda_{c}\right)\right]=n \cdot\left[B_{\odot}\left(\lambda_{l}\right)\right]
$$

where $n=1.0054$ (Allen, 1963) ${ }^{5}$. Therefore, (3.20) can be rewritten as:

$$
E\left(\lambda_{l}\right)_{\left[B_{\odot}\left(\lambda_{l}\right)\right]}=\left(1.0054 \cdot I\left(\lambda_{l}\right)\right)_{\left[B_{\odot}\left(\lambda_{c}\right)\right]}-\beta \cdot I\left(\lambda_{c}\right)_{\left[B_{\odot}\left(\lambda_{c}\right)\right]}
$$

where the scaling factor of the continuum is computed after multiplying the on-line image by $n=1.0054$ to account for the difference in the continuum emission at the wavelengths of the on- and off-line images. With (3.21) the resulting emission line image is expressed in ppm of the continuum intensity at $526.0 \mathrm{~nm}$ at the center of the solar disk.

\section{Discussion}

As for the determination of the calibration factor $\xi\left(\lambda_{i}\right)$, it is crucial to have the flat fields for the line and continuum images as close in time to the respective images as possible in order to have $\Upsilon_{i} \approx 1$. The closer to 1 the value of $\Upsilon_{i}$, the more reliable the conversion to ppm of the continuum intensity at the center of the solar disk. This allows to get rid of the attenuation effects of the terrestrial atmosphere on the incoming signal. The other requirement for the validity of (3.21) is to have also the continuum images very close in time to the on-line images to reduce mainly the effects of the sky variability. An example of the effect produced by not fulfilling the latter requirement is shown in Figure 3.3. The first image (top left) corresponds to an on-line image (40 sec exposure time) treated with the closest off-line image ( 6 sec exposure time), the time between both images being 2 min (Case "a"). The second one (top right) corresponds to the same on-line image but treated with another continuum image taken $\sim$ 8 min before (Case "b"). Note the different radial variation of the sky intensity around the solar disk between the corresponding pair of images (on- and off line). In the bottom panel of the same figure, the radial cuts performed at position angle $P=0^{\circ}$ are shown for comparison, i.e., where no green line signal is expected (Section 4.1). The two solid lines correspond to the radial cuts of the images shown on top of the Figure (cases "a" and "b"), while the dots correspond to the radial cut of the same on-line image treated with a continuum taken $\sim 14$ min before (Case "c"). There is practically no difference in the radial profile between cases "b" and "c", this fact revealing the

\footnotetext{
${ }^{5}$ For the estimation of $n$, we used linear interpolation between the intensities at the center of the solar disk with spectrum lines smoothed, at $500.0 \mathrm{~nm}$ and $550.0 \mathrm{~nm}$ (as given by Allen, 1963).
} 

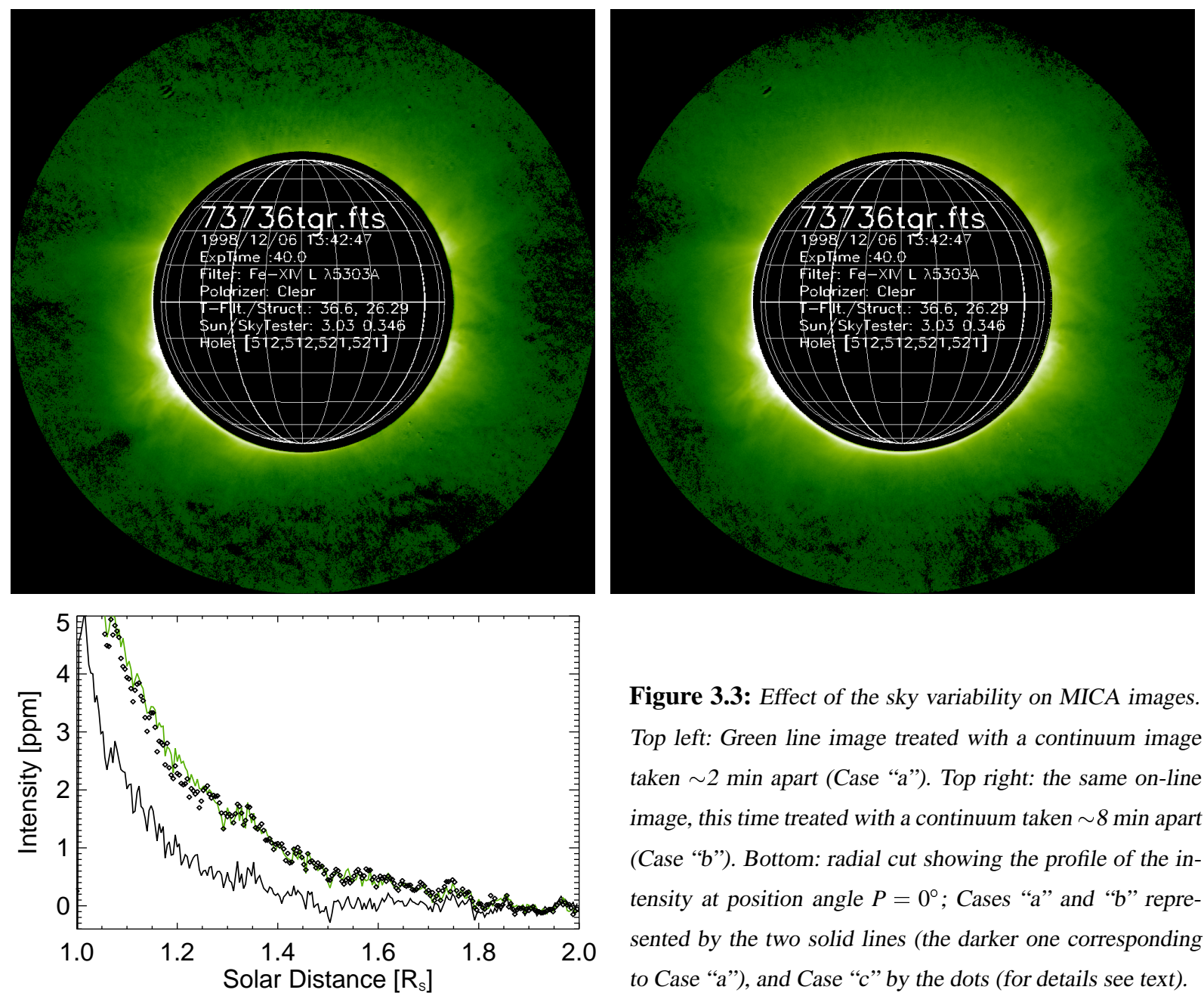

Figure 3.3: Effect of the sky variability on MICA images. Top left: Green line image treated with a continuum image taken $\sim 2$ min apart (Case "a”). Top right: the same on-line image, this time treated with a continuum taken $\sim 8$ min apart (Case "b"). Bottom: radial cut showing the profile of the intensity at position angle $P=0^{\circ}$; Cases "a" and " $b$ " represented by the two solid lines (the darker one corresponding to Case "a"), and Case "c" by the dots (for details see text).

random nature of the sky variability around the solar disk. Note also that close to $2 \mathrm{R}_{\odot}$ the intensity is zero (by definition), since at that distance is where the $\beta$ factor is defined (see Section 3.3.4).

On the other hand, it is important to mention the importance of (3.19) in regard to the flatfielding procedure. As it can be seen, it serves not only to convert the number of counts/sec detected by the CCD to ppm, but also to compensate the different gain every pixel usually has.

\subsubsection{On the determination of the scaling factor $\beta$}

The first attempt to scale the continuum images (Equation 3.11) was to take the average value of a $M p x l \times M p x l$ box $(M=100)$ defined at the upper right corner of the field of view, where the emission line signal is likely to be negligible (Epple, 1997). However, the final processed MICA images showed a residual asymmetry around the center (see, e.g., bottom left panel on Figure 3.4) due to a likely relative misalignment of the on- and off-line filters, not completely corrected by the flatfielding process. In order to solve this problem, I used a different method to obtain the scaling factor $\beta$ for the continuum image. Instead of considering $\beta$ constant for the whole field of view 
(scalar), I assumed that $\beta=\beta(\varphi)$ at $r=2 \mathrm{R}_{\odot}$ (one-dimensional array), $\varphi$ being the polar angle in a reference system with origin in the center of the field of view ${ }^{6}$. By assuming the same angular dependence from the edge of the field of view towards the center of the image a synthetic image $\beta$ is created, $\beta_{i, j}$ being the scaling factor corresponding to the homologous pixels in the continuum image. In the bottom right panel of Figure 3.4 the improvement can be appreciated. For better comparison of both cases, a circular cut made in the aforementioned images at $2 \mathrm{R} \odot$ is shown in the top panel of the same figure.

\footnotetext{
${ }^{6} \mathrm{By}$ using matrix notation, let $l_{i, j}$ be the on-line image and $c_{i, j}$ the continuum one, the pair $(i, j)$ representing the pixel position. Let define $r_{\infty}=\left\{(i, j):\left(i^{2}+j^{2}\right)^{1 / 2}=2 R_{\odot}\right\}$. By expressing the pixel positions in polar coordinates in a system centered at the center of the field of view (i.e, using " $r, \varphi$ " instead of " $i, j$ "), the scaling factor is obtained from the quotient of the intensity profiles of the on- and off-line images at $2 \mathrm{R}$ as $\beta_{r, \varphi}=\frac{l_{r_{\infty}, \varphi}}{c_{r_{\infty}, \varphi}}$.
}

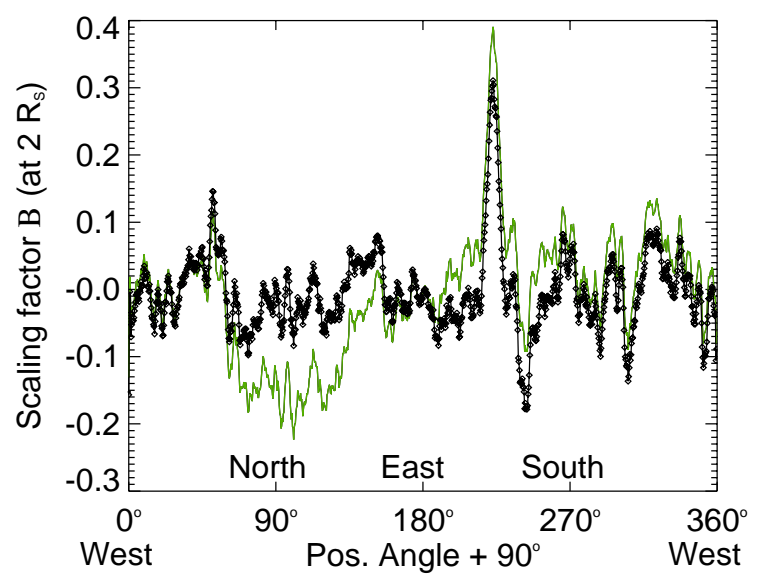

Figure 3.4: The image on the left panel (bottom) shows a treated green line image obtained using a scalar scaling factor $\beta$ computed according to Epple (1997). In the right panel (bottom), the same image is shown, this time the image was obtained using an angular dependent $\beta$ factor obtained at $2 R_{\odot}$ (for details see text). The intensity profile obtained by making a circular cut at $2 R_{\odot}$ in the respective images is shown on the left (top). Note the depression around $90^{\circ}$ (heliographic north) in the curve corresponding to the image treated with $\beta=$ constant (green solid line).
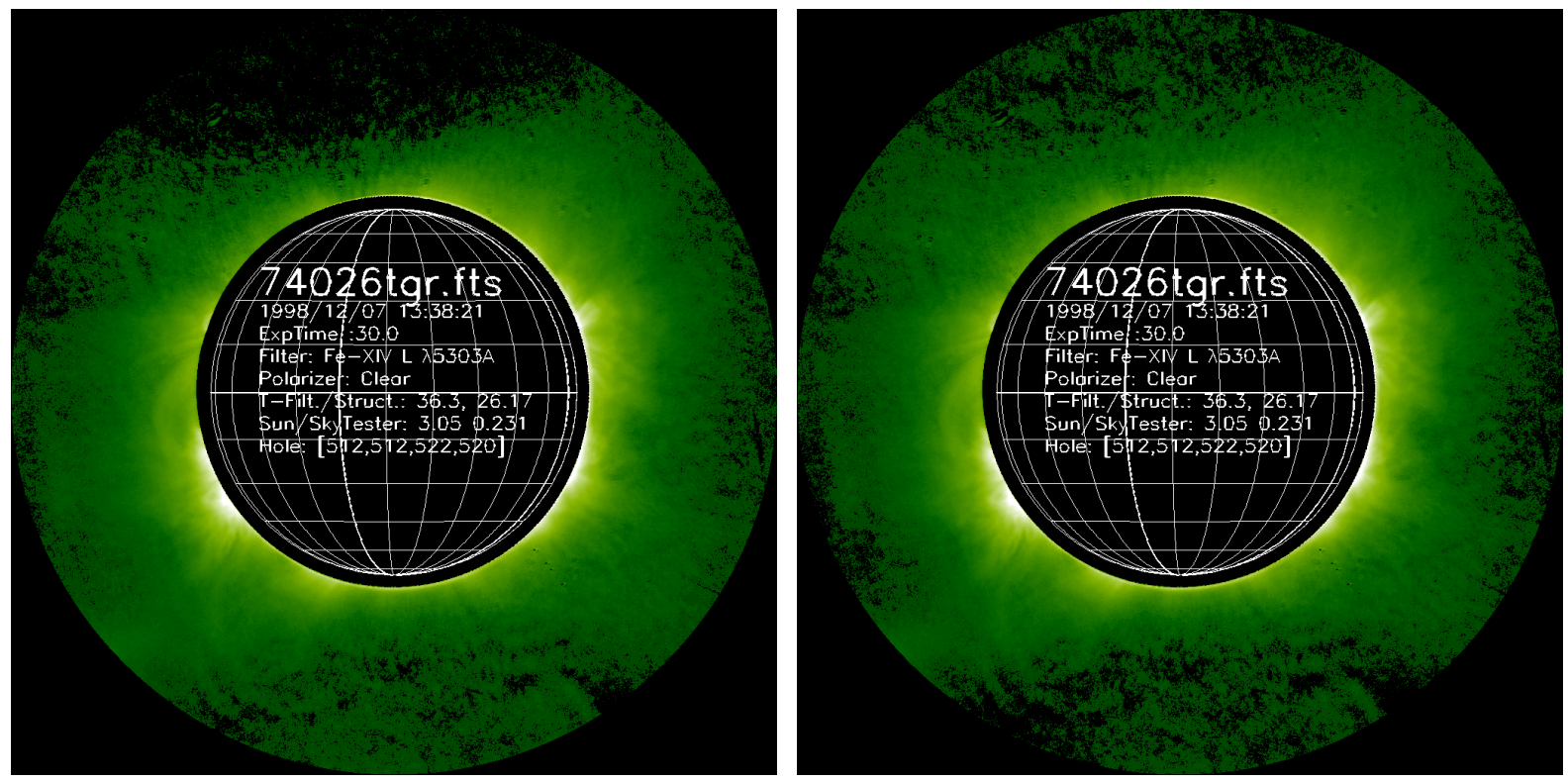

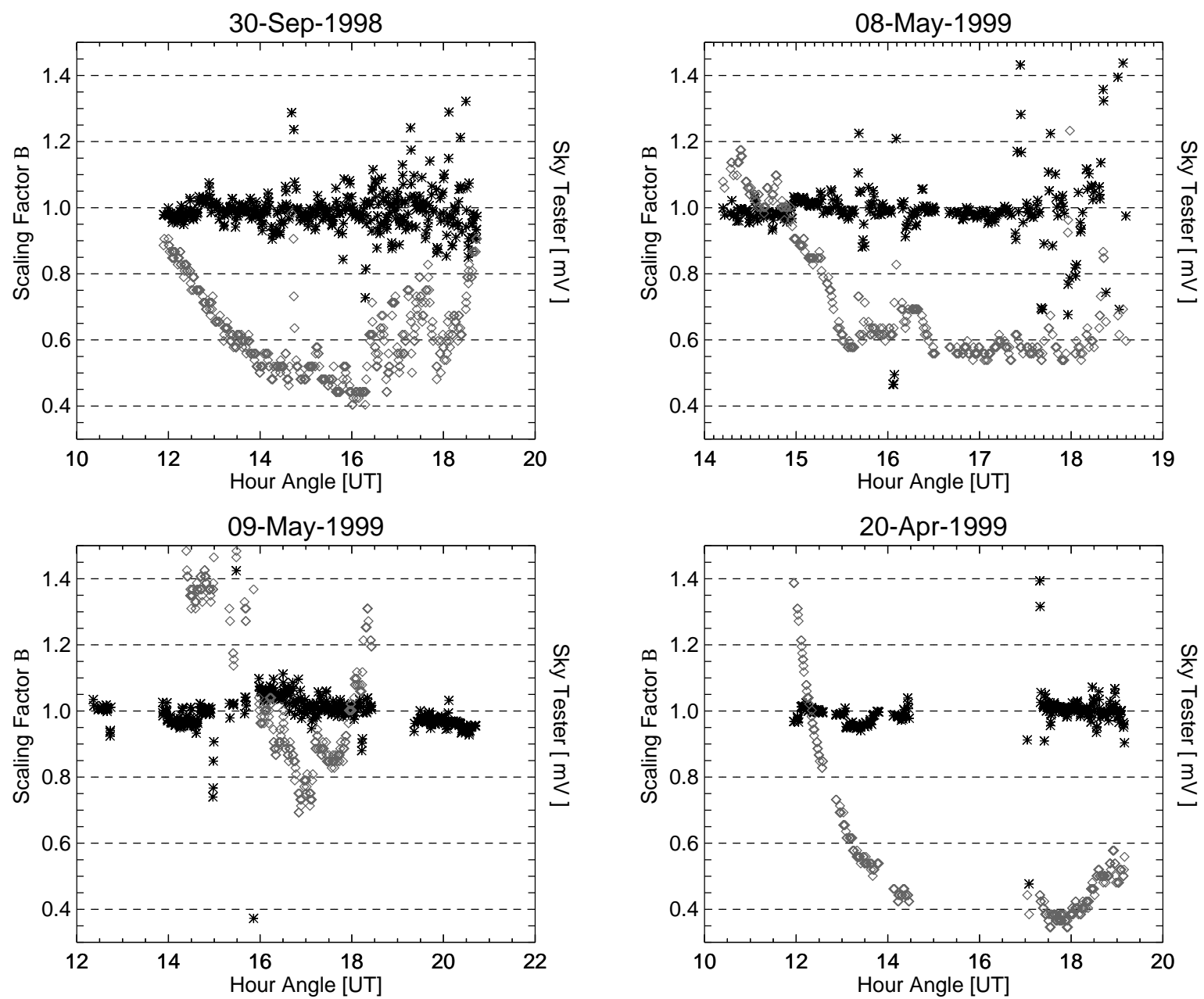

Figure 3.5: Example of the behaviour of the scaling factor $\bar{\beta}$ with different sky conditions. For reference, the values of the sky tester (in gray) recorded at the moment of the exposure are also shown. Note how the dispersion of $\bar{\beta}$ around its mean value increases as the sky variability increases (represented by the increasing dispersion of the sky tester values).

\section{Discussion}

It was shown in the theoretical discussion at the beginning of the Chapter that $\beta$ accounts for the correction of residual sky light due to changes in the sky brightness between the on- and off-line images. One would expect on coronal days then, that the average value of $\beta$, i.e. $\bar{\beta}$, be randomly distributed around 1 . In the next sections this topic will be addressed in deeper detail. At this stage, it's only worth to show an example of the observational confirmation of such an asseveration. The average $\beta$ values obtained along typical days at El Leoncito are shown in Figure 3.5 . 


\subsubsection{On the effects of the transmittance of the Earth's atmosphere $\tau_{i}$}

The validity of the equations (3.9) and (3.10) depends upon the constancy of the transmittance of the Earth's atmosphere, i.e., $\tau(\lambda, t)$, during the time lapse between the respective open (on- and off-line) and closed (flat field) door images. Moreover, the factors $\Upsilon_{l}$ and $\Upsilon_{c}$ in (3.9) and (3.10) have been previously introduced to explicitly account for the departure from the ideal case. Since an instantaneous determination of the terms involved in the definition of $\Upsilon_{l}$ and $\Upsilon_{c}$ is not possible, in this subsection we outline a rough estimate for these factors and show an example of the error of the calibration based on these estimates ${ }^{7}$.

The transmittance of the Earth's atmosphere $\tau(\lambda, t)$ at time $t$ may be written as (see Appendix B.2)

$$
\tau(\lambda, t)=e^{-K(\lambda, t)}
$$

the exponent $K(\lambda, t)$ being

$$
K(\lambda, t)=\left(k_{r}(\lambda, t) X_{h}+k_{a}(\lambda, t) X_{h}+k_{o z}(\lambda, t) l\right) \cdot X(t)
$$

where $k_{r}(\lambda, t)$ is the extinction coefficient due to pure Rayleigh scattering (Equation B.4), $k_{a}(\lambda, t)$ denotes the extinction coefficient due to aerosols and water droplets (Equation B.5), $k_{o z}(\lambda, t)$ depicts the extinction coefficient due the ozone absorption (Appendix B.2.3), and $X_{h} \cdot X(t)$ and $l \cdot X(t)$ represent the optical path length for the process under consideration, i.e., the air mass (see Appendix B.1). On the one hand, molecular diffusion depends on temperature and pressure. These quantities are subject to the relatively slow variations set by large-scale meteorological patterns. On the other hand, the ozone absorption is a more stable component showing only small seasonal variations (see Appendix B.2.3). It is thus only the aerosol and water droplet component $k_{a}(\lambda, t)$ which accounts for the short-time variability of the total extinction component. Therefore, we assume in the following that the temporal dependence is significant only in regard to the aerosols and water content of the atmosphere. For that reason, we will refer to $k_{a}$ simply as the extinction coefficient, unless the distinction have to be mentioned explicitly to avoid confusion.

Thus, the time-dependent problem to be addressed is that of the slow and rapid changes of the transmission of the Earth's atmosphere. The visible effect of the slow-varying transmission coefficient due to a slow-varying extinction coefficient is the growth in size and brightness of the solar aureola from morning until late afternoon as convection caused by solar heating mixes aerosols produced near ground level. On the other hand, for the especial case of constant extinction coefficient, the decreasing ${ }^{8}$ (increasing ${ }^{9}$ ) air mass (hour-angle dependent) also produces a visible effect in the size and brightness of the solar aureola. In the rapid-varying case, the effect can be clearly observed by the great variability of the brightness of the aureola (see, e.g., figures 2.9 and 3.5). Therefore, which is the point that separate slow from rapid changes? When do the changes of the extinction coefficient begin to affect the calibration of the images? When does the air mass difference between the open and closed door images begin to be significant? In order to answer these questions, we have to remember one of the hypotheses made in regard

\footnotetext{
${ }^{7}$ The error introduced in both line and continuum images is due to the neglect of the parameters $\Upsilon_{\mathfrak{l}}$ and $\Upsilon_{c}$. It will be shown in Section 3.3 .6 how the scaling factor $\bar{\beta}$ accounts for the instantaneous estimation of the error.

${ }^{8}$ From sunrise until local noon.

${ }^{9}$ From local noon until sunset.
} 
to the derivation of (3.9) and (3.10), i.e., the time elapsed between the respective open (on- and off line) and close door (flat fields) images. This time lapse is referred as $\delta t$ for the present discussion. For a numerical estimation of the error introduced in the calibration by the changes of the transmission coefficient occurred during $\delta t$, we introduced in (3.9) and (3.10) the factors $\Upsilon_{l}$ and $\Upsilon_{c}$, respectively. They are defined as

$$
\Upsilon_{i}=\frac{\tau\left(\lambda_{i}, t\right)}{\tau\left(\lambda_{i}, t+\delta t\right)}=\frac{e^{-K\left(\lambda_{i}, t\right)}}{e^{-K\left(\lambda_{i}, t+\delta t\right)}}=e^{\delta K\left(\lambda_{i}\right)}
$$

provided $K\left(\lambda_{i}, t+\delta t\right)=K\left(\lambda_{i}, t\right)+\delta K\left(\lambda_{i}\right)$ with $K\left(\lambda_{i}, t\right)$ given by Equation 3.23. The subindex $i$ is used here to indicate either line, i.e., $l$, or continuum, i.e., $c$. Having in mind the above discussion about the variability of the different components of the total extinction coefficient, $\delta K\left(\lambda_{i}\right)$ can be written as

$$
\delta K\left(\lambda_{i}\right)=\left(k_{r}\left(\lambda_{i}, t\right) X_{h}+k_{a}\left(\lambda_{i}, t\right) X_{h}+k_{o z} l\right) \cdot \delta X+X(t) \cdot \delta k_{a}
$$

where $\delta X$ and $\delta k_{a}$ account for the air mass difference, i.e., the difference in zenith distance, and change of the water droplet and aerosol content, respectively, between the time at which the open door image is taken $(t)$ and the time of the respective flat field $(t+\delta t)$. Therefore,

$$
\Upsilon_{i}=e^{\left(k_{r}\left(\lambda_{i}, t\right) X_{h}+k_{a}\left(\lambda_{i}, t\right) X_{h}+k_{o z} l\right) \cdot \delta X+X(t) \cdot \delta k_{a}}
$$

Equation (3.25) allows us to answer the aforementioned questions. The error will be minimum when $\Upsilon_{i} \rightarrow 1$, i.e., $\delta k_{a} \rightarrow 0$ and $\delta X \rightarrow 0$. The first condition depends upon the time variability of the sky conditions during the observing time. Further, it does not matter how big the long-time scale changes of the water droplets and aerosol content are, provided the short-time scale changes can be considered constant within the time lapse between the open door images and their respective flat fields (i.e., $\delta t$ ). On the other hand, the closer to 0 the value of $\delta t$, the closer to 0 the value of $\delta X$. In other words, the air mass difference can also be minimized by taking the respective flat fields as close as possible to the open door images.

\section{Discussion}

Let us see an example of the error introduced by the variation of the transmission of the Earth's atmosphere along the observing time in a sample of green continuum MICA observations. Figure 3.6 (left panel) shows the difference in zenith distance $X$ between the continuum images and the flat fields used for their calibration as function of the hour angle $t$ along a typical day. The oscillatory (wave-like) shape of the curve is due to the fact that the flat fields are selected according to the shortest time distance to the continuum image to be calibrated. In this way, the continuum images sometimes precede the corresponding flat fields, while others are the flat fields to be used those that precede the continuum images. In order to visualize the effect of the error introduced by the finite value of $\delta k_{a}$, a reliable estimate of the instantaneous extinction coefficient $k_{a}$ is necessary. As will be shown in Section 3.3.6, its instantaneous value cannot be accurately calculated. However, a crude estimate can be done assuming its constancy along the day, by using the Ångström turbidity formula (see Appendix B.2.2). Figure 3.6 (center panel) shows the values of $k_{a}$ as estimated with (B.5) for different values of the parameters $\alpha$ and $\beta$ of such equation. Another 

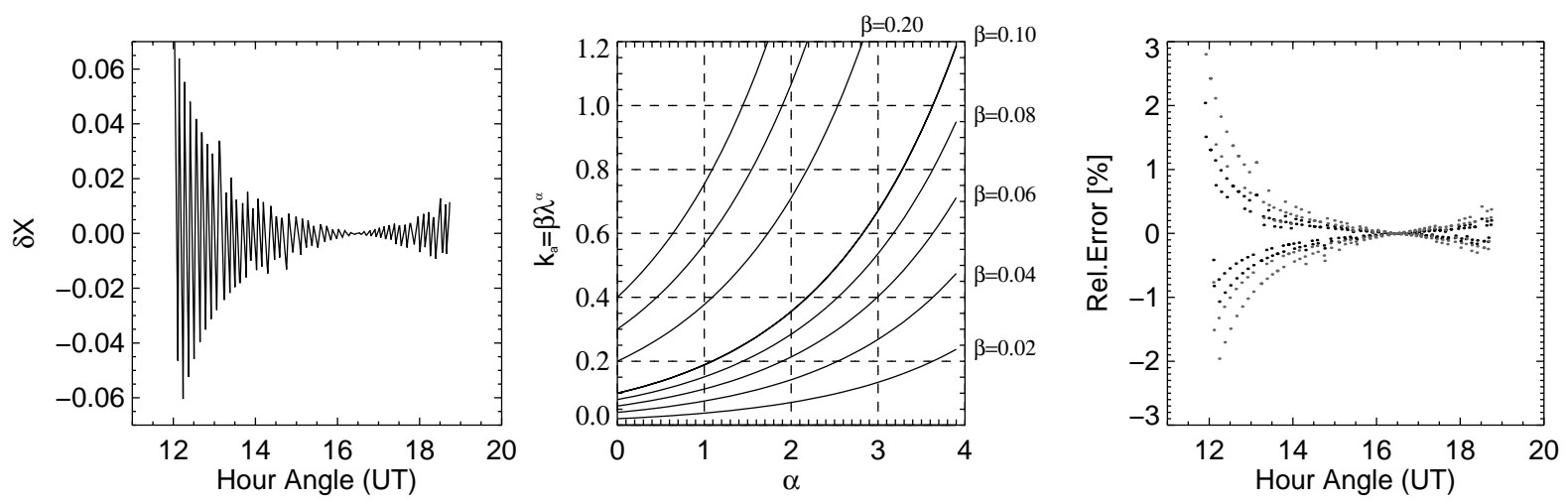

Figure 3.6: Left: Example showing the air mass difference between the continuum observations and the respective flat fields used for calibration. Center: Estimate of the extinction coefficient $k_{a}$ with the Ångström turbidity formula, i.e., $k_{a}=\beta \cdot \lambda^{\alpha}$, for $\lambda=530.3 \mathrm{~nm}$ (Appendix B.2.2). Right: Estimate of the relative error along a typical day for two different values of atmospheric cleanliness $\left(k_{a}=0.2\right.$ and $\left.k_{a}=0.4\right)$.

parameter needed is that of the Rayleigh extinction factor $k_{r}$. According to (B.4) is $k_{r}=0.11$ at $\lambda=530.3 \mathrm{~nm}$. The extinction coefficient due to the Ozone absorption $k_{o z}$ may be estimated for the corresponding time of the year with the help of tables B.1 and B.2. Finally, the pressure correction $X_{h}$ is evaluated at $h=2400$ m, i.e., the altitude at "El Leoncito", using the formula $X_{h}=\exp (h / 8 \mathrm{~km})$ (see Appendix B.1). Figure 3.6 (right panel) shows an estimation of the relative error, i.e., $\left(\Upsilon_{c}-1\right) \cdot 100$, the corresponding continuum images would have under two extreme values of atmospheric cleanliness (i.e., $k_{a}=0.2$ and $k_{a}=0.4$ ) provided the constancy of the aerosols and water droplets content.

From this brief analysis, it is clear that when the extinction coefficient keeps constant along the day, the error in the calibration of the MICA images is larger at larger zenith distances and can be easily estimated. However, the time variability of the extinction coefficient makes such an estimate difficult. In the next subsection we address this problem in deeper detail and show how the scaling factor $\beta$ can be used to estimate the time-dependent error introduced in the calibration.

\subsection{6 $\bar{\beta}$ as an estimate of the accuracy of the calibration in regard to the transmittance of the Earth's atmosphere}

In this subsection, we show how the individual average values of the scaling factor $\beta$ can be used to quantify the error introduced in every "treated image" by neglecting the parameters $\Upsilon_{l}$ and $\Upsilon_{c}$ in (3.9) and (3.10), establishing a limit to the maximum accuracy allowed by the calibration method.

For a numerical estimation of the error introduced in the calibration by the combined effects of $\delta X$ and $\delta k_{a}$ (Section 3.3.5) occurred during $\delta t$, we introduced in (3.9) and (3.10) the factors $\Upsilon_{l}$ and $\Upsilon_{c}$, respectively. Thus, assuming there is no emission line contribution at distances sufficiently far from the solar limb., i.e, where $\beta$ is defined, it can be easily demonstrated from (3.12) that 


$$
\bar{\beta}=\frac{\Upsilon_{l}}{\Upsilon_{c}}
$$

Therefore, by using (3.26) in (3.12) we have

$$
E\left(\lambda_{l}, t\right)=\Upsilon_{l} \cdot\left(C^{f}\left(\lambda_{l}, t\right)-C^{f}\left(\lambda_{c}, t+\delta t\right)\right)
$$

in such a way that $\Upsilon_{l}-1 \doteq \varepsilon_{l}$ is representative of the error in the calibrated intensity of the emission line. Since the exact determination of $\Upsilon_{l}$ is not possible (see Section 3.3.5), the average value of the scaling factor $\beta$ can be used as an estimator (at least to order 0 ) of such a parameter since

$$
\varepsilon_{l}=\bar{\beta} \Upsilon_{c}-1 \stackrel{\Upsilon_{c} \rightarrow 1}{\rightarrow} \bar{\beta}-1
$$

\section{Example}

The different $\bar{\beta}$ values obtained for the observations of several days are shown in Figure 3.5. For comparison, the brightness of the solar aureola (i.e., sky brightness around the Sun) as detected by the sky tester device (Section 2.5.2) is represented in the same graph. The Figure clearly shows the correlation existent between the variability of the brightness of the solar aureola and $\bar{\beta}$. It can thus be noted that the accuracy of the calibration, i.e., $\bar{\beta}-1$, strongly depends on the short-time variability of the extinction coefficient (represented in the graph by the changing values recorded by the sky tester) and not on the global intensity of the solar aureola (see, e.g., the graph corresponding to May 9, 1999 in Figure 3.5).

\subsubsection{On the determination of the normalization factor $f_{k}$}

As mentioned in Section 2.3.1, the filter passband depends on the filter temperature. For the MICA filters, the temperature dependence is given by the manufacturer to be $0.168 \AA / K$. By assuming a Gaussian profile for the online MICA filters, with the technical specifications given by the manufacturer (Table 2.1), it is possible to simulate the transmission profile and its dependence with temperature.

On the other hand, the emission line profile $\varphi(\lambda)$ must also be known in order to correctly compute the normalization factor $f_{k}$ (Equation 3.17). Many factors influence the shape and width of spectral lines, these coming under the general heading of line broadening. Briefly, the major contributions to the shape and width of spectral lines are i) Natural broadening, which is associated with the finite lifetime of the excited states and the Heisenberg's uncertainty principle, ii) Pressure Broadening, which arises due to collisions of absorbing/emitting atoms (ions) with other atoms and/or ions, iii) Thermal broadening, which is due to the different thermal velocities individual atoms (ions) can have, and iv) Turbulent broadening, which is due to macroscopic movements of the emitting atoms (ions). The first two are in the form of a Lorentz or "damping profile", whilst the last two are Gaussian in shape. The combination of Lorentz and Gaussian profiles is known as Voigt profile. As suggested by Pecker et al. (1954), and later confirmed by Billings (1956), for the particular cases of the green and red coronal lines, the line profile broadening is in both cases primarily thermal. Nevertheless, one is always confronted with the possibility that turbulent macroscopic motions contribute to the width of line profiles. Since both thermal and turbulent 


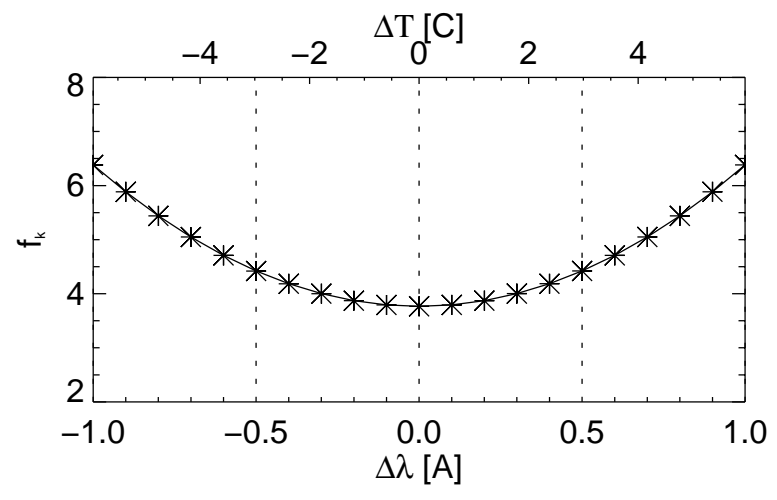

Figure 3.7: Estimate of the normalization factor $f_{k}$ for the green line filter. For details see text.

macroscopic motions produce Gaussian-like profiles, the use of a Gaussian-like profile for the simulation of the emission line profile is justified. The other point is the assumption that the emitting features do not move along the line of sight. A motion would cause a Doppler shift (either blue- or red-shift) of the central wavelength. A quick estimation using the well-known formula of the Doppler shift, i.e., $\Delta \lambda / \lambda=v / \mathrm{c}$, tells that velocities of $\pm 30 \mathrm{~km} / \mathrm{sec}$ along the line of sight are enough to produce $\pm 0.04 \mathrm{~nm}$ shift of the central wavelength at $530.3 \mathrm{~nm}$. Furthermore, since at optical wavelengths the corona is an optically thin medium, the emitted radiation represents an integration of the emission along the line of sight. This fact makes the election of a fixed line profile all across the field of view even less desirable.

Since the MICA instrument does not have spectroscopic capabilities, a very crude and constant estimation of the line profile must be chosen. Therefore, based on the work of Koutchmy (1983) we assume a Gaussian-like shape centered at $530.3 \mathrm{~nm}$ with FWHM of $0.09 \mathrm{~nm}$ for the green line for the computation of the factor $f_{k}$.

Therefore, with the Gaussian profiles assumed for both the emission line and filter transmission and Equation (3.17), the factor $f_{k}$ was computed at different temperatures. The result of such a calculation is shown in Figure 3.7. The shape of the curve obtained suggests the fitting of the points by a second degree polynomial. The function obtained, i.e.,

$$
f_{k}=2.616(T-36 C)^{2}+3.767
$$

evaluated at the temperature $T$ the filters have at the time of the exposure of the on-line image can thus be used to estimate easily the normalization factor $f_{k}$. The reliability of the estimate of $f_{k}$ is implicitly proven in Section 3.5, by the goodness of the results obtained when comparing MICA green line calibrated intensities with green line intensities recorded by another ground-based calibrated instrument.

\subsection{The practical real-time reduction scheme}

In the present section a scheme of the real-time reduction algorithm is outlined. All the images that enter in the reduction scheme are "well exposed", which means that the raw images are on-line checked (i.e., during the development of the sequence) either for saturation or underexposure, any of these resulting in automatic autocorrection of the exposure time and retaking of the respective image. 
The main steps of the reduction scheme can be summarized as follows (the numbers appearing as supraindex indicate the number of step):

1. Offset and Dark Charge Correction: $I_{j}^{1}\left(\lambda_{i}\right)=I_{j}\left(\lambda_{i}\right)-D\left(t_{j}\right)$ where $I$ denotes any image in the sequence (either on-line, off-line or flats), $j$ the number of image within the sequence, and $D\left(t_{j}\right)$ the dark image ${ }^{10}$ corre- $^{-}$ sponding to an exposure of $t_{j} \sec$ (i.e., the exposure time of $I_{j}$ ).

2. Exposure Time Normalization: $I_{j}^{2}\left(\lambda_{i}\right)=I_{j}^{1}\left(\lambda_{i}\right) \cdot t_{j}^{-1}$

3. Flatfielding and Conversion to ppm: Let $\lambda_{l}$ denote the emission line wavelength and $\lambda_{c}$ the wavelength of the continuum emission, and let us use $F$ instead of $I$ to indicate de flat fields (processed according to the previous two items as well). Then

$$
\begin{gathered}
I_{j}^{3}\left(\lambda_{l}\right)=1.0054 \cdot \frac{I_{j}^{2}\left(\lambda_{l}\right)}{F^{2}\left(\lambda_{l}\right) \cdot \xi\left(\lambda_{c}\right)} \\
I_{j}^{3}\left(\lambda_{c}\right)=\frac{I_{j}^{2}\left(\lambda_{c}\right)}{F^{2}\left(\lambda_{c}\right) \cdot \xi\left(\lambda_{c}\right)}
\end{gathered}
$$

4. Scaling of the continuum: Due to a not exact parallelism between the on- and off- line filters, the image of the occulter appears in the on- and off-line images at not exactly the same position (1-2 pixels at most displaced of each other). Thus, before scaling and subtraction, the shift of the continuum images is necessary in order to spatially match the on-line images. The procedure is straightforward, once the position of the center of the occulter in both images is found. Due to gravitational bending of the structure ${ }^{11}$ (its effect producing a drift of the occulter in the field of view along the day) the center determination is done for every image. Afterwards, the scaling factor $\bar{\beta}$ for each pair of images is determined according to

$$
\beta_{r, \varphi}=\frac{\left[I_{j}^{3}\left(\lambda_{l}\right)\right]_{r_{\infty}, \varphi}}{\left[I_{j}^{3}\left(\lambda_{c}\right)\right]_{r_{\infty}, \varphi}}
$$

5. Continuum and scattered light removal: $E_{j}\left(\lambda_{l}\right)_{\left[B_{\odot}\left(\lambda_{c}\right)\right]}=I_{j}^{3}\left(\lambda_{l}\right)-\beta_{r, \varphi} \cdot I_{j}^{3}\left(\lambda_{c}\right)$

6. Line profile correction: As aforementioned, assuming a Gaussian-like profile for the filter transmission and line profile, the integrated intensity is calculated according to (3.17), i.e.,

$$
E_{0}(t)_{\left[B_{\odot}\left(\lambda_{c}\right)\right]}=E_{j}\left(\lambda_{l}\right)_{\left[B_{\odot}\left(\lambda_{c}\right)\right]} \cdot f_{k}
$$

where

$$
f_{k}=\frac{\left[\int Q_{l}(\lambda, T) d \lambda\right]\left[\int \varphi(\lambda, t) d \lambda\right]}{\int \varphi(\lambda, t) \cdot Q_{l}(\lambda, T) d \lambda} \approx 2.616(T-36 C)^{2}+3.767
$$

$T$ indicates the temperature of the filters at the time of the exposure.

\footnotetext{
${ }^{10}$ If both a short $(D(t \rightarrow 0))$ and a long $(D(t))$ dark images are specified within the sequence, the offset and the so-called differential dark are computed by making the two dark exposures $\left(D(t \rightarrow 0)\right.$ and $\left.D\left(t_{j}\right)\right)$ belonging to the straight line defined by $D=o f f s e t+D_{d} \cdot t$, where $D_{d}$ denotes the differential dark. Then, the dark to be used for the offset and dark charge correction of each of the images is obtained from $D\left(t_{j}\right)=$ off set $+D_{d} \cdot t_{j}$. If dark exposures are not specified within the sequence, the previous offset and differential dark computed is considered for the evaluation of the dark frame.

${ }^{11}$ Fortunately the movement is largely elastic and there is not a significant amount of hysteresis present.
} 
7.Miscellaneous: Finally a set of steps is performed, such as:

7.a Shifting: The position of the occulter in the processed images depends on the time of the day at which the respective images were taken (due to gravitational bending of the structure). For that reason, the treated images are finally shifted to a common center, i.e., at the exact geometrical center of the CCD chip.

7.b Rotation: Afterwards, the processed images are rotated in order to show the heliographic north up (west at right).

7.c Grid: Then, a scaled Sun grid depicting the heliographic coordinates is embedded in the images.

\subsection{Comparison with calibrated data}

Scans in position angle of the green line emission corona $(530.3 \mathrm{~nm})$ at $1.15 \mathrm{R}_{\odot}, 1.25 \mathrm{R}_{\odot}$, and $1.35 \mathrm{R}_{\odot}$ are daily made with the NSO/Sacramento Peak Emission Line Coronal Photometer, fed by the $40 \mathrm{~cm}$ coronagraph at the John W. Evans Facility. The instrument and its operation have been described in some detail by Fisher (1973) and Smartt (1982). Briefly, the intensity of the green corona is recorded at 120 points around the limb, i.e., every $3^{\circ}$ in position angle, by chopping between the corona and the sky at a rate of $100 \mathrm{kHz}$. A complete scan in position angle takes approximately 5 minutes. The recorded intensities in millionths of the brightness of the center of the solar disk, i.e., ppm, are obtained by calibrating the system at disk center through a neutral density filter. These daily scans are published in the Solar Geophysical Data.

Green line scans of several days along the year were kindly provided by R. Altrock ${ }^{12}$ in order to compare the results of MICA calibration with another calibrated source and test the hypotheses made in the design of the observation routine.

\subsubsection{Results}

Several days were selected for comparison. In order to have similar data sets the following was taken into account:

1. Each Sacramento Peak scan takes approximately 5 minutes. Therefore, the three scans corresponding to the three solar distances span about 15 minutes. The MICA image corresponding to the respective day results of the median of the images taken in the time lapse between the first and last Sacramento Peak scan.

2. Circular cuts around the occulter (1 pixel wide in radial direction) at $1.15 \mathrm{R}_{\odot}, 1.25 \mathrm{R}_{\odot}$, and $1.35 \mathrm{R}_{\odot}$ were made in the full resolution MICA green line treated images selected ${ }^{13}$.

\footnotetext{
${ }^{12}$ These observations are a joint production of the Air Force Geophysics Laboratory and National Solar Observatory at Sacramento Peak.

${ }^{13}$ It must be noted that the solar diameter as measured from Earth changes along the year due to the varying distance Sun-Earth, and so the field of view (fov) of the MICA instrument. The nominal inner fov at $1.05 \mathrm{R}$. corresponds to equinox time (solar semi-diameter: 959.63 arcsec; Allen, 1963). In local summer time (December 21st), the inner fov corresponds to $\sim 1.032 \mathrm{R}_{\odot}$ (solar semi-diameter: 943.89 arcsec), while in local winter time (June 21st) is $\sim 1.068 \mathrm{R}_{\odot}$ (solar semi-diameter: 975.93 arcsec). Therefore, this is taken into account when computing solar distances.
} 
In figures 3.8 and 3.9 we have displayed the MICA intensity profiles resulting from the circular cuts described above. In the same plots the corresponding Sacramento Peak scans are superimposed for comparison. The MICA images were selected such that they correspond to different times of the year in order to test the validity of the hypotheses made for the calibration (Section 3.2). The graphs show that the MICA green line calibrated images in terms of part per million (ppm) of the continuum intensity at $526.0 \mathrm{~nm}$ at the center of the solar disk are in good agreement with those obtained with the NSO/Sacramento Peak Emission Line Coronal Photometer.

It can also be seen that the scatter in intensity of MICA images close to the limb, i.e., at $1.15 \mathrm{R}_{\odot}$, is much less than that at greater solar distances, i.e., at $1.35 \mathrm{R}_{\odot}$. This is likely due to the relative level of the green line intensity with respect to the background intensity. The reduction technique used on MICA images, which involves subtraction of continuum images to model the sky and continuum emission intensity, could be a possible explanation to the scatter observed.

\subsection{Concluding remarks on the calibration}

The objective of the last part of this Chapter is twofold. At first, I summarize the constraints existent when calibrating coronagraph observations. Secondly, I give some comments about the interpretation of the features observed in the final images.

\subsubsection{Constraints to the calibration}

\section{Residual light}

How can we make sure that a rapid change either in the continuum emission or in the sky brightness did not occur in the time lapse between the on- and off-line image? To answer this question, running differences of continuum images must be performed in parallel to the normal reduction procedure, to put un upper limit to the amount of continuum and sky contamination in the final images. During times of low solar activity, no detectable continuum residual in the running difference continuum images was observed. However, at times of fast dynamical events, as it will be shown in the next Chapter, the changes in the continuum are important. This fact makes the interpretation of coronal images more difficult.

\section{Line Profile}

Many kinds of mass motions occur in the solar corona (Newton et al., 1995), especially at the triggering and during the development of dynamical events. Consequently, the determination of the absolute position and width of the emission line at those particular times is very problematic, leading to an unknown uncertainty in the estimation of the normalization factor $f_{k}$. The line broadenings are non thermal and probably due to the turbulent conditions in the flaring plasma as long as there is observational evidence of the energy release in the flare site. In the decay phase of flares, nonthermal velocities are either not observed or are present at very low levels. One possible interpretation most commonly accepted regards the overall line profile as a superposition of various Doppler-shifted components. 

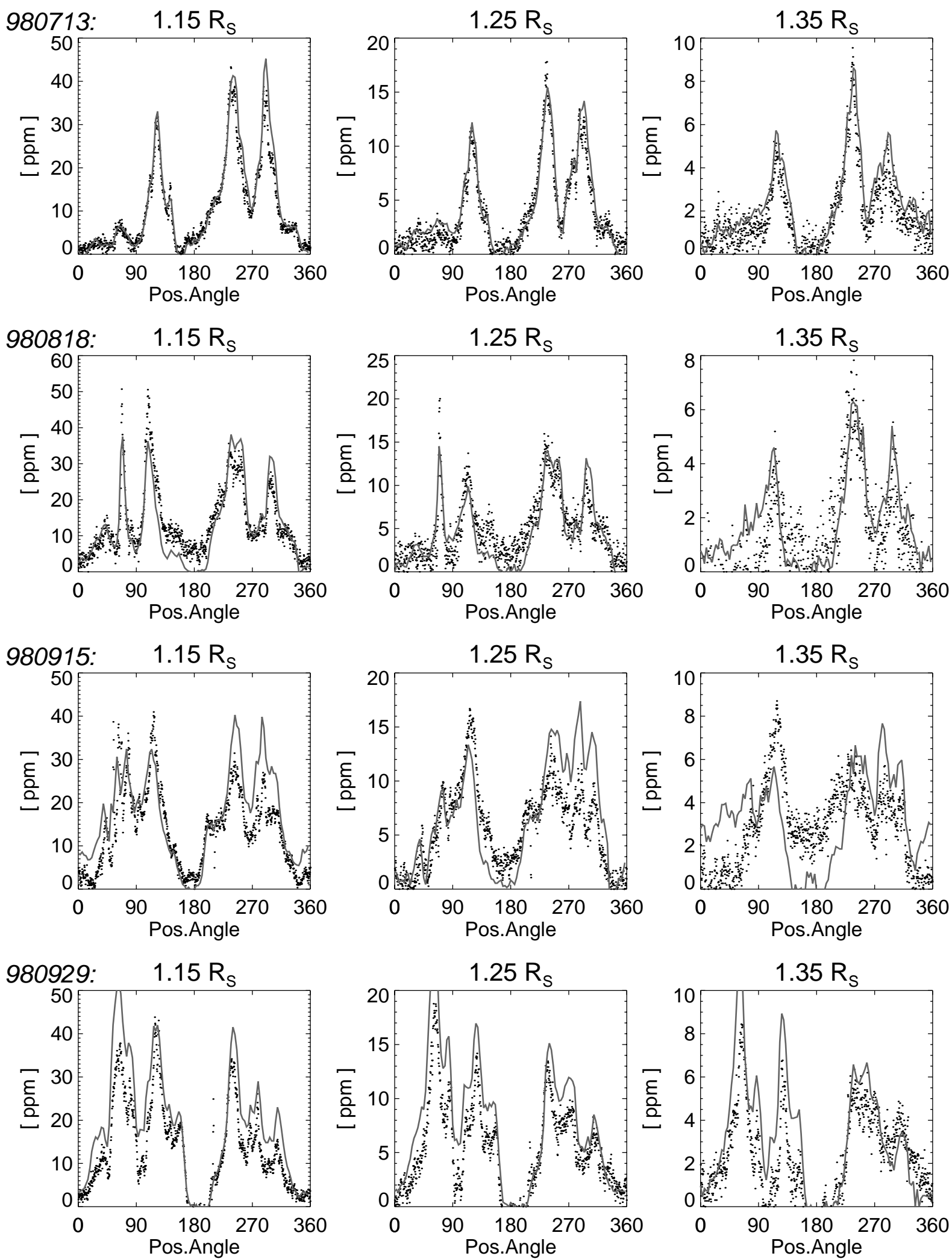

Figure 3.8: Comparison of the Fe XIV green line intensity recorded by MICA (dots) with that recorded by the NSO/Sacramento Peak Emission Line Coronal Photometer (solid line) on July 13, August 18, September 15 and September 29, 1998 at three different solar distances. 

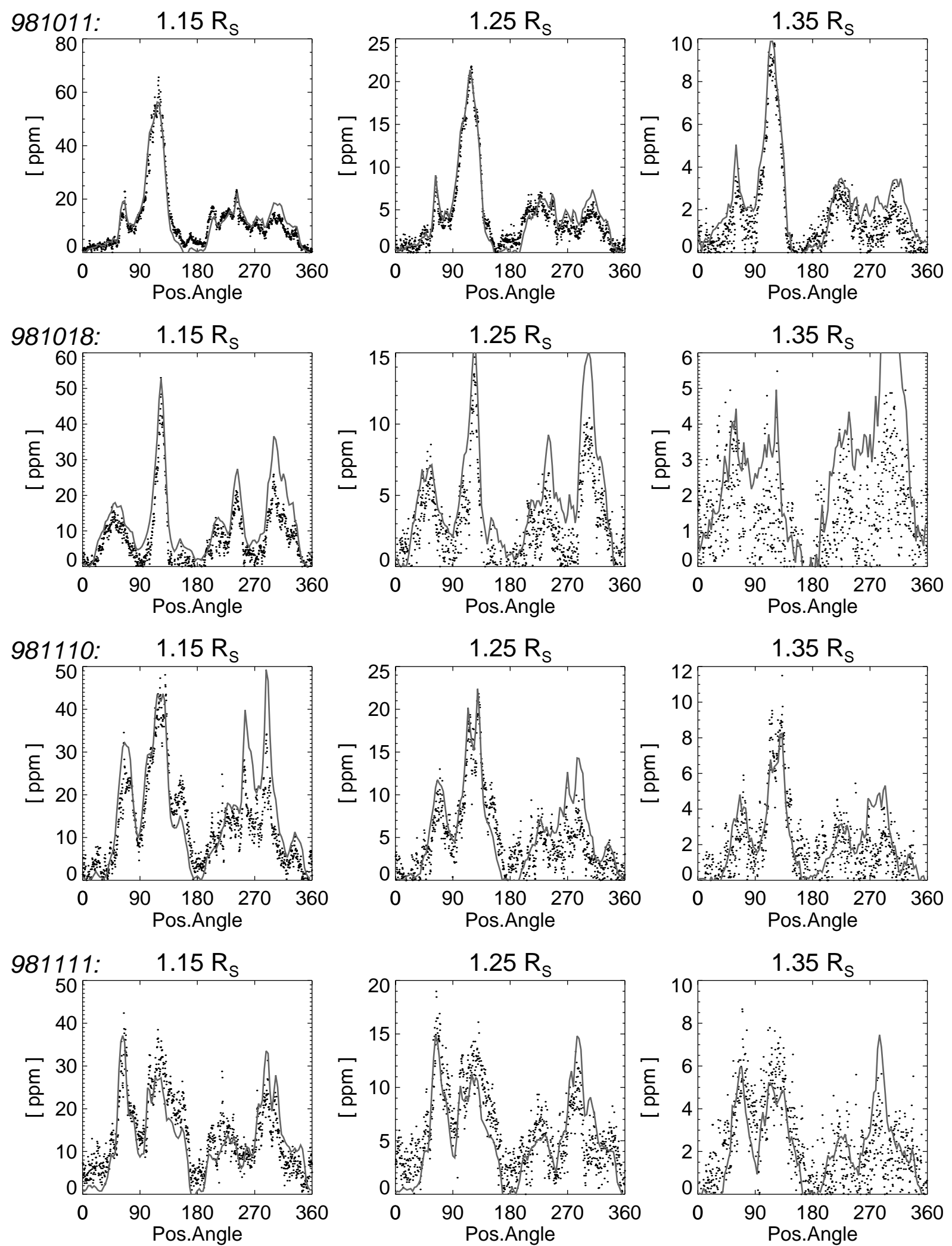

Figure 3.9: Comparison of the Fe XIV green line intensity recorded by MICA (dots) with that recorded by the NSO/Sacramento Peak Emission Line Coronal Photometer (solid line) on October 11, October 18, November 10 and November 11, 1998 at three different solar distances. 
This superposition is believed to be a result of an integration along the line of sight of various distinct, unresolved loop structures (or part of). An alternative view (Antonucci et al., 1986) is that the observed line broadening is due to a superposition of Doppler shifted line profiles arising from distinct plasma flows originating within a single loop structure. Regardless of the actual reason, the observed excess broadening of the line profiles accounts for some uncertainty in the MICA calibration.

\subsubsection{On the interpretation of coronal images}

To finish this Chapter, a summary of the aspects to have in mind to avoid erroneous interpretation when analyzing coronal images is given. They were already mentioned by Dunn (1971). According to the experience gained we summarize them as

- Atmospheric effects on the spatial resolution: Depending on the spatial resolution of the instrument, seeing effects may play an important role due to the long exposure time necessary for the observation of the faint coronal lines. This effect can be avoided by space-based observations. In the case of MICA, seeing effects play no role since its spatial resolution $\sim 8$ arcsec is much lower than the normal seeing conditions at the site.

- Guiding errors:They are also accentuated during long exposures due to seeing effects.

- Smearing: During the evolution of very fast dynamical events, the long exposure time required produce smearing of the moving features in the image. For example, a coronal feature moving at $800 \mathrm{~km} / \mathrm{sec}$ in the plane of sky travels $32000 \mathrm{~km}$ ( 46 arcsec) in $40 \mathrm{sec}$ (typical exposure time for on-line images). Given the spatial resolution of the MICA instrument, this represents $\sim 10$ pixels. Therefore, thin faint features may not be discernible. Moreover, it is an important factor to have in mind when analyzing the morphology of fast dynamical events.

- Movements along the line of sight: An emission feature moving towards the observer at $20 \mathrm{~km} / \mathrm{sec}$ has a Doppler shift of $\sim 0.35 \AA$ A . Due to the narrow band of the filters used, the wavelength of the emission will be out of the passband, making that feature invisible. Misinterpretation of the images can occur.

- Residual sky background: The error introduced by the effects of rapid changes in the sky brightness produce a flickering background when observing the images as a movie.

- Residual continuum background: Fast changes in the continuum emission cannot be completely removed. In MICA's case, e.g., those occurring in time lapses less than the time existent between two successive continuum images. Thus, analysis of continuum images is critical for the right interpretation of the on-line images (Chapter 4).

- Optical thickness and projection effects: Since the corona is optically thin, different features along the line of sight cannot be resolved. For example, the crossing of two loops at different spatial location along the line of sight, is viewed as a bright point. This bright point cannot be discerned from a bright point indicating 
a local high density. Further, projection effects makes more difficult to track the coronal features back to photospheric levels. 



\section{Chapter 4}

\section{The Observations at Short Time Scales}

We now look at the interpretation of the different features observed in coronagraph green line images. In the introduction we briefly outline the main features and excitation mechanisms responsible for the green line emission. The morphological characteristics of the green line corona are also outlined. The second section describes a mathematical common framework for the analysis of MICA images. Several possible scenarios are shown with practical examples. The examples comprise the analysis i) in coronal regions with no underlying activity, i.e., above the socalled quiet Sun, and ii) during the development of a fast dynamical event. The emphasis is placed on the correct interpretation of the observed features in the green line images obtained with the MICA telescope, i.e., whether they really arise from the emission of $\mathrm{Fe}^{13+}$ ions or are due to continuum emission not properly subtracted. Further, a morphological description of dynamic phenomena, i.e., coronal transients, from a kinematic point of view is also given for the dynamical event presented.

\subsection{Introduction}

The emission corona at visible wavelengths is observed to be highly structured and variable. It comes mostly from forbidden lines of multiply ionized atoms. Iron is one of the most prolific sources of the emission corona, two examples being the well known green and red emission lines at $530.3 \mathrm{~nm}$ and $637.4 \mathrm{~nm}$, respectively. The iron emission is intimately linked to the presence of magnetic fields that are generated inside the Sun and reach out into the extended hot corona.

The identification of the coronal emission lines in the visible has a fascinating history (see, e.g., Chapter 1; Zirin, 1988). The crucial step was done by Grotrian when in a short note in Die Naturwissenschaften (Grotrian, 1939), he pointed out that the existence of coronal lines in stellar objects implied that these lines arise under conditions of high temperature. Further, he showed that there was an almost exact correspondence between the wavelength of the red coronal line at $637.4 \mathrm{~nm}$ and that derived from Edlén's measurements of the separation of the levels of different J-values in the ground state of $\mathrm{Fe}^{9+}$. Spurred by Grotrian's discovery, Edlén (1942) searched for further coincidences and found them for the coronal lines at $332.8 \mathrm{~nm}$ and $408.6 \mathrm{~nm}$, which he could identify with transitions in $\mathrm{Ca}^{11+}$ and $\mathrm{Ca}^{12+}$. Many other strong coronal lines were also identified by him, by extrapolating wavelengths along isoelectronic sequences. Among them he identified the nowadays well known green coronal line at $530.3 \mathrm{~nm}$, the brightest of all coronal emission lines in the visible spectral range. It was found to arise from the forbidden transition $3 p{ }^{2} P_{3 / 2} \rightarrow{ }^{2} P_{1 / 2}$ of $\mathrm{Fe}^{13+}$ ions, the ionization potential being $355 \mathrm{eV}$. The emissivity of the green line peaks at a temperature of about $1.8 \times 10^{-6} \mathrm{~K}$, i.e., the temperature at which the relative abundance of the ion with respect to the neutral atom is maximal (see Figure 4.1).

The excitation mechanisms of this line are not very well known, especially with reference to the relative contributions of collisional and radiative processes at various solar altitudes and position angles. Waldmeier (1942) 


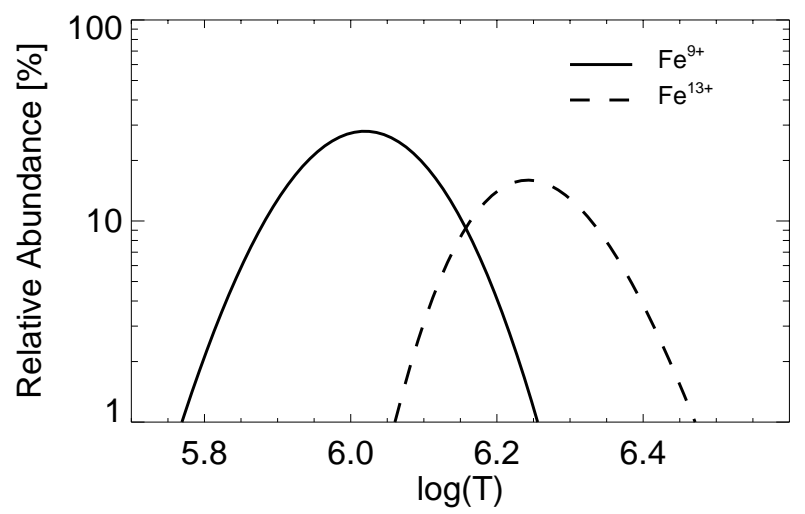

Figure 4.1: Ionization equilibrium of $\mathrm{Fe}^{13+}$ and $\mathrm{Fe}^{9+}$ ions. From Arnaud \& Rothenflug (1985).

found that the variation of the line intensity is much stronger than that of the continuum, and it depends on the local structure of the corona. Later, he found that for distances greater than $1.8 \mathrm{R} \odot$, the ratio of line to continuum intensity becomes constant, which is an indication of dominant radiative excitation. Towards smaller distances collisional excitation becomes progressively more important, and at $\sim 1.3 \mathrm{R}_{\odot}$ both mechanisms contribute the same amount. Many authors studied the excitation mechanisms of the green line (see, e.g., Raju \& Singh, 1987; Raju et al., 1991; Raju \& Desai, 1993). It was generally found that the excitation mechanism is mainly collisional in the inner regions (up to $\sim 1.4 \mathrm{R}_{\odot}$ ) where the density is high, and radiative outwards.

\section{Overall morphology of the green corona}

Among the identifiable structures in the green coronal images at times of minimum solar activity (see, e.g., Figure 4.2), one can distinguish (after Schwenn et al., 1997):

- Bright closed loop systems centered at latitudes between $30^{\circ}$ and $45^{\circ}$ in both hemispheres, their helmet-like outer extensions being bent toward the equator plane. These mid-latitude loop systems are usually found above the position of magnetic neutral lines ${ }^{1}$ in photospheric magnetograms. By analogy to the structures in white light images, these structures are called streamers. They are seen to remain stable on time scales of several days.

- At low latitudes, a more diffuse pattern is usually visible, well separated from the mid-latitude streamers, showing a very pronounced variability.

- Coronal holes located mainly above the polar caps. They are recognized as those regions with lack of green line emission.

As solar activity increases, local bright enhancements in form of loops above active regions are observed, while the coronal holes are gradually disappearing.

\footnotetext{
${ }^{1}$ The photospheric magnetic neutral line is that where the radial component of the magnetic field is zero, i.e., where the magnetic field is parallel to the surface.
} 


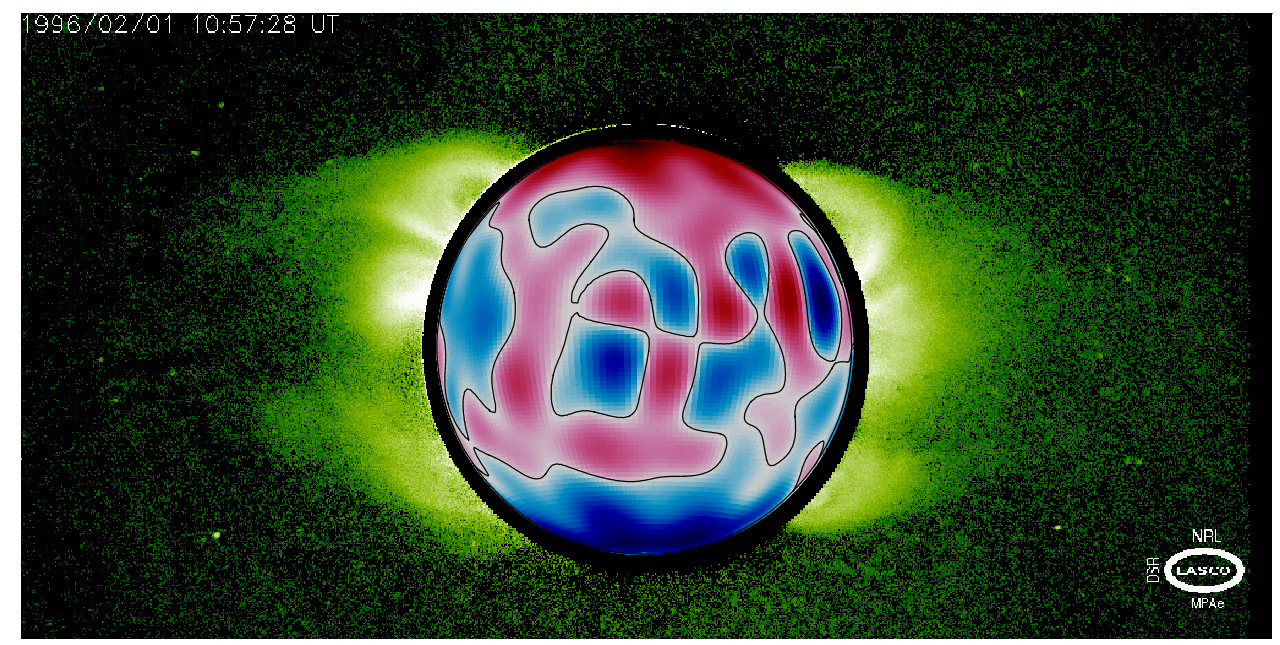

Figure 4.2: Composite of LASCO/C1 image and map of the photospheric magnetic field observed from the Wilcox Solar Observatory from Standford University. The C1 image was taken in the green coronal emission line of 13 times ionized iron (usually referred to as Fe XIV line) at a wavelength of $530.3 \mathrm{~nm}$. From Schwenn et al. (1997).

\section{Time series analysis of coronal observations}

Analysis of time series of the emission corona in the long time scale (e.g., for more than one solar rotation) reveals the periodic occurrence of emission patterns (see, e.g., Chapter 5; Lewis et al., 1999; Sime et al., 1989; Antonucci \& Dodero, 1979 and references therein). In shorter time scales (ranging from hours to seconds) a wide spectrum of both oscillatory and dynamic phenomena is observed to occur. The study of short-timescale intensity fluctuations in coronal structures is strongly motivated, e.g., by coronal heating theories that involve wave interactions as, for example, resonant heating by Alfvén waves (Steinolfson and Davila, 1993).

There are very few imaging observations however, that document oscillatory phenomena in the solar corona while there is a larger number of indirect inferences based on quasiperiodic patterns observed in temporal analyses of line profiles. Nevertheless, the physical mechanisms behind oscillatory phenomena in the solar corona, which cover a wide range of wavelengths and periods, are still virtually unidentified, mainly because of poor spatial resolution and insufficient time cadence. Further, a number of oscillatory phenomena are known to occur inside the Sun (e.g, the so-called $p$-modes ${ }^{2}$ ) as well as near the solar surface (e.g., running penumbral waves ${ }^{3}$, Moreton waves $^{4}$, and Sun quakes ${ }^{5}$ ) but there is no observational evidence yet whether and how these oscillatory phenomena

\footnotetext{
${ }^{2}$ p-modes are resonant pressure waves travelling inside the Sun (Leibacher et al., 1985).

${ }^{3}$ Running penumbral waves (RPWs) were first reported by Zirin \& Stein (1972) and by Giovanelli (1972). RPWs are bright circular waves moving outward from the umbra-penumbra border to the exterior edge of the penumbra with a period of about 200 sec. Theories of the RPWs are reviewed by Chitre (1991).

${ }^{4}$ The phenomenon was first reported by Moreton (1961), Athay \& Moreton (1961), Ramsey \& Smith (1966) in H $_{\alpha}$ images. They are also known as "flare waves". They consist of the propagation of a semi-circular front that takes place typically up to a distance of the order of $10^{6} \mathrm{~km}$ from its source, a flare, with a constant velocity in the range of [330-4200] km/sec (Smith \& Harvey, 1971). For a recent study the reader is referred to Thompson et al., 1999 and references therein.

${ }^{5}$ Sun quakes were first shown by Kosovichev \& Zharkova (1998) using data from the Michelson Doppler Imager (MDI/SOHO, Scherrer
} 
might couple to the magnetic structures seen in the corona.

On the other hand, phenomena involving changes in the magnetic topology (i.e., dynamic phenomena) are widely studied. Many of these coronal transients end up in ejections of material, usually referred to as coronal mass ejections (CME). Some of them may potentially be geo-effective, because of the ejected material and the associated coronal dynamics (Gosling, 1993). These dynamic phenomena are now thought to be the primary mechanism for removing magnetic helicity from the interior of the Sun (see, e.g., Low, 1996) Hence, they provide the fundamental mechanism by which the large-scale corona reorganizes and sheds magnetic flux, and hence may play a central role in the solar cycle. The causal relation between these coronal transients and flares and prominence eruptions is not yet settled. Therefore, an understanding of the mechanisms responsible for the CME initiation has long been a primary research goal of solar physics.

\subsection{Mathematical description of typical scenarios observed in coronal im- ages}

As will be shown later, the changes in the continuum intensity, i.e., electron density changes, in case of some dynamical events can be faster than the time difference between on- and off-line images. Hence, in order to address the study of such phenomena, it is important to take into account the observational constraints of the instrument, namely the time difference between continuum and on-line images. In order to set a common framework for the study of such events with the MICA telescope, we now outline a general mathematical description of the possible scenarios one can observe in MICA images. This mathematical formulation will serve as a guide to be followed in any study carried out with the MICA telescope or a coronagraph with similar observational constraints.

Let $S$ be the region of interest defined by the set of points (x,y), and $R$ the area surrounding the region $S$ (see Figure 4.3). Let $I_{S}$ and $I_{R}$ be the average intensity of the respective regions. We want to characterize $S$ in terms of the average intensity of $R$ with the region $S$ excluded from it. The cases of interest are i) $I_{S}<<I_{R}$ and ii) $I_{S}>>I_{R}$. The notation $<<$ is used here to denote an appreciable difference in intensity between both regions. Now let us address the problem of the temporal evolution of both regions and their implications in terms of the calibration.

Let $L\left(t_{i}, x, y\right)$ and $C\left(t_{j}, x, y\right)$ denote the intensity in $p p m$ in the "treated" and continuum images respectively, as function of the pixel position $(x, y), t_{j}$ the time at which the continuum image was acquired, and $t_{i}$ the time at which the on-line image used to obtain $L\left(t_{i}, x, y\right)$ was taken. Let $l\left(t_{i}, x, y\right)$ be the green emission line contribution and $c\left(t_{i}, x, y\right)$ the continuum contribution in the on-line image taken at $t_{i}$, and $\Delta t$ the time-lapse during which the condition lasts (either $I_{S}>>I_{R}$ or $I_{S}<<I_{R}$ ). Let us also define $\Upsilon_{S}(t)$ as the instantaneous position of the center of the region of interest $S$ along successive images, and let $\rho$ indicate the spatial resolution of the instrument. Then, for analysis purposes we consider two cases. The first one will be the stationary case, defined as that where the displacement of the region of interest $S$ during the time interval $\left[t_{j-1}, t_{j}\right]$ is negligible, i.e., $\Delta \mathrm{\Upsilon}_{S}<\rho$. The second et al., 1995). They are produced by very energetic flares. 
one will be the nonstationary case, that means, that one where the displacement of $S$ during the aforementioned interval is finite, i.e., $\Delta \Upsilon_{S}>\rho$.

The Stationary Case: $\Delta \Upsilon_{S}<\rho$

Let us assume first for the following discussion that the sky variability remains negligible all across the field of view between the on- and off-line images and that the instrumental straylight contributes the same amount at both wavelengths. For simplicity, we discard the arguments indicating the pixel position $(x$ and $y)$.

In view of the hypotheses made and having in mind Equation 3.12 with $\beta=\Upsilon_{l}=\Upsilon_{c}=1$, we can write the average intensity observed in the region $S$ as:

$$
L_{S}\left(t_{i}, t_{j}\right)=l_{S}\left(t_{i}\right)+c_{S}\left(t_{i}\right)-C_{S}\left(t_{j}\right)=l_{S}\left(t_{i}\right)+\delta c_{S}\left(\left|t_{i}-t_{j}\right|\right)=l_{S}\left(t_{i}\right)+\varepsilon_{S}\left(\left|t_{i}-t_{j}\right|\right)
$$

where $\varepsilon_{S}$ denotes the residual continuum intensity in the region of interest in the treated image. There is no way to estimate the exact value of $\varepsilon_{S}$, but an upper limit can be established under certain assumptions. Let us suppose for example that the continuous intensity changes monotonically in time at least during the time lapse $\left[t_{j-1}, t_{j}\right]$, i.e, during the time elapsed between two continuum images. In other words, $\partial c / \partial t]_{\left[t_{j-1}, t_{j}\right]}>0$ (or $<0$ ). Then,

$$
\left|\varepsilon_{S}\left(\left|t_{i}-t_{j}\right|\right)\right|<\left|\gamma_{S}\left(\left|t_{j-1}-t_{j}\right|\right)\right|
$$

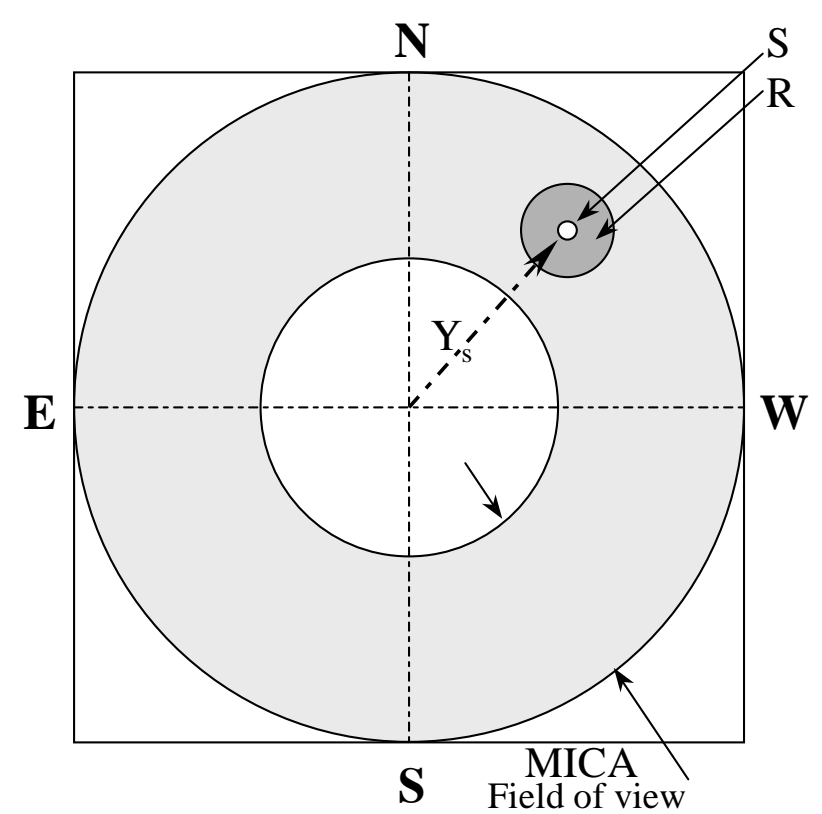

Figure 4.3: Sketch of the MICA field of view showing the generic regions $S$ and $R$. The region $S$ is intended to characterize the position $\left(\Upsilon_{S}\right)$ and extent of a particular coronal feature in terms of its surrounding environment $(R)$ in order to estimate the amount of continuum contamination in that particular region. The continuum contamination in MICA "treated" images arises from the finite time difference between on- and off-line images. For details see text. 
where $t_{j-1}<t_{i}<t_{j}$. The greek letter $\gamma_{S}$ was used to denote the intensity difference between two continuum images in the region of interest $S$, i.e.,

$$
\gamma_{S}\left(\left|t_{j-1}-t_{j}\right|\right)=C_{S}\left(t_{j-1}\right)-C_{S}\left(t_{j}\right)
$$

Using (4.2) we can now rewrite (4.1) as

$$
\begin{aligned}
& \left.L_{S}\left(t_{i}, t_{j}\right) \leq l_{S}\left(t_{i}\right)+\gamma_{S}\left(\left|t_{j-1}-t_{j}\right|\right) \quad \text { if } \quad \partial c / \partial t\right]_{\left[t_{j-1}, t_{j}\right]}>0 \\
& \left.L_{S}\left(t_{i}, t_{j}\right) \geq l_{S}\left(t_{i}\right)+\gamma_{S}\left(\left|t_{j-1}-t_{j}\right|\right) \quad \text { if } \quad \partial c / \partial t\right]_{\left[t_{j-1}, t_{j}\right]}<0
\end{aligned}
$$

Therefore, the absolute value of $\gamma_{S}$, i.e., $\left|\gamma_{S}\right|$, allows us to estimate an upper limit of the error introduced in the treated images by monotonic changes of the continuum intensity during the observing sequence provided i) the sky variability is negligible at least during the period $\left[t_{j-1}, t_{j}\right]$, ii) the instrumental straylight amounts the same contribution in the calibrated on- and off- line images, and iii) the continuum intensity increase (decrease) monotonically during the interval $\left[t_{j-1}, t_{j}\right]$ as mentioned before.

As shown in Section 3.3.6, the small changes in the sky intensity during the time between on- and off-line images (i.e., changes in the transmittance coefficient of the Earth's atmosphere) are taken into account by means of the correction factor $\beta$ in Equation 3.12, provided $\beta$ has no radial dependence (see, e.g., Section 3.3.4). Moreover, $\beta$ accounts also for the wavelength dependence of the instrumental straylight. In that case, (4.1) and (4.4) can be rewritten as

$$
L_{S}\left(t_{i}, t_{j}\right)=l_{S}\left(t_{i}\right)+\varepsilon_{S}\left(\left|t_{i}-t_{j}\right|\right)+\varepsilon_{S}^{\prime}\left(\left|t_{i}-t_{j}\right|\right)
$$

and

$$
L_{S}\left(t_{i}, t_{j}\right) \leq l_{S}\left(t_{i}\right)+\gamma_{S}\left(\left|t_{j-1}-t_{j}\right|\right)+\varepsilon_{S}^{\prime}\left(\left|t_{i}-t_{j}\right|\right)
$$

where $\varepsilon_{S}^{\prime}$ denotes the change in intensity between the on- and off-line image in the region of interest $S$, due to the combined effect of the sky variability and instrumental straylight. Under the aforementioned assumption of no radial dependence for $\beta^{6}$, it is possible to estimate an upper limit to the amount of residual scattered light $\varepsilon_{S}^{\prime}$ if we consider that the changes in the continuum intensity are much lower than the changes in the sky intensity during the time lapse $\left[t_{i}, t_{j}\right]$. In that case, we can estimate $\varepsilon_{S}^{\prime}$ using a region of the images sufficiently far from the solar disk (where the line signal is considered to be negligible). Hence,

$$
\left.\left.\varepsilon_{S}^{\prime}\left(\left|t_{i}-t_{j}\right|\right)=\varepsilon_{\infty}^{\prime}\left(\left|t_{i}-t_{j}\right|\right)=L\left(t_{i}\right)\right]_{\infty}-C\left(t_{j}\right)\right]_{\infty}
$$

The subindex $\infty$ is used here to denote a region in the outermost part of the images.

Let us suppose now that the continuum intensity changes in time in an arbitrary way (in other words, the time constant ${ }^{7} \tau_{c}$ is relatively short, i.e., $\left.\tau_{c}<\left|t_{j}-t_{j-1}\right|\right)$. This is the typical case occurring during most of the coronal transients. In this case there is no way to quantify the amount of contamination in the treated images. A careful analysis of temporal sequences of individual on- and off-line images is thus needed to give the right interpretation to the treated images.

\footnotetext{
${ }^{6}$ The hypothesis of no radial dependence for $\beta$ implies that the changes in the sky intensity do not depend on the distance from the Sun's center, at least in the region subtended by the field of view of the telescope.

${ }^{7}$ Time lapse during which the continuum intensity can be considered constant.
} 


\section{The Nonstationary Case: $\Delta \Upsilon_{S}>\rho$}

In the previous discussion it was assumed that the emitting features remain spatially stationary during the interval $\left[t_{j-1}, t_{j}\right]$. At the time of the reference continuum $t_{j}$, the features producing the emission are at the same relative spatial position they had at the time of the on-line image, i.e., $\Upsilon_{S}\left(t_{j}\right)=\Upsilon_{S}\left(t_{i}\right)$. Let us suppose now that the emitting features move while changing their intrinsic intensity such that $\Upsilon_{S}\left(t_{j}\right)-\Upsilon_{S}\left(t_{j-1}\right) \doteq \Delta \Upsilon_{S}>\rho$. In this case, we can rewrite (4.5) as

$$
L_{S}\left(t_{i}, t_{j}, \Upsilon_{S}\left(t_{i}\right)\right)=l_{S}\left(t_{i}, \Upsilon_{S}\left(t_{i}\right)\right)+c_{S}\left(t_{i}, \Upsilon_{S}\left(t_{i}\right)\right)-C_{S}\left(t_{j}, \Upsilon_{S}\left(t_{j}\right)\right)+\varepsilon_{S}^{\prime}\left(\left|t_{i}-t_{j}\right|\right)
$$

where with the argument $\Upsilon_{S}$ we indicate the actual position of the emitting feature. It is straightforward to see that the continuum emission produced by the moving feature does not cancel out when subtracting the off-line image from the on-line one. Hence, the intensity enhancement observed at the $\Upsilon_{S}\left(t_{i}\right)$ position in the treated image is highly contaminated by the continuum emission, which is not properly subtracted. Moreover, all the on-line images taken during the time elapsed between two continuum images, i.e., during the period $\left[t_{j-1}, t_{j}\right]$, will have a common (and likely visible) feature seen in "absorption" at the position $\Upsilon_{S}\left(t_{j}\right)$. In other words,

$$
I_{S}\left(t_{i}, \Upsilon_{S}\left(t_{j}\right)\right)<I_{R}\left(t_{i}, \Upsilon_{S}\left(t_{j}\right)\right) \quad \forall t_{i}: t_{j-1}<t_{i}<t_{j}
$$

The intensity enhancement observed at $t_{i}$ will be observed to drift towards the absorption common feature at $\Upsilon_{S}\left(t_{j}\right)$ as $t_{i} \rightarrow t_{j}$. Since it is not possible in the nonstationary case to put an upper limit to the amount of continuum contamination, the aforementioned behaviour of the moving feature has to be kept in mind when analyzing the morphology of dynamical events (see Section 4.4.1).

\subsection{Stationary coronal features}

As already mentioned, the green line emission characteristic of the regions with no underlying activity, i.e., far from active regions, is that of a diffuse pattern subjected to a great variability (Schwenn et al., 1997). Moreover, above certain structures, known as quiescent filaments (prominences), the green line pattern is characterized by a pronounced decrease in intensity. Therefore, it is our intention in this section to look for the correct interpretation of the features observed in the regions mentioned above in MICA green line images. The analysis is performed in those regions where the spatial changes undergone by the features are less than one pixel during at least the time lapse $\left[t_{j-1}, t_{j}\right]$, i.e., the framework developed in the previous section for the stationary case is considered.

\subsubsection{The green line above quiescent prominences}

Quiescent prominences are long-lived, thin sheets with comparatively high gas density (of the order of 5 . $10^{10} \mathrm{~cm}^{-3}$ ) suspended in the lower corona by magnetic field tension (see, e.g., Tandberg-Hanssen, 1995). They are always situated above the magnetic neutral line separating regions of opposite polarity in the large-scale photospheric field. However, not all neutral lines are associated with prominences. The nature of the field supporting 
the prominence material remains still unclear. They can extend up to a substantial fraction of a solar radius, i.e., a few $10^{5} \mathrm{~km}$, and be quite stable, showing little change over periods of months. In $\mathrm{H}_{\alpha}$ emission, quiescent prominences appear on the solar disk as dark filaments, because of the enhanced absorption of the photospheric light. On the other hand, they show up in emission above the limb. Eventually, quiescent prominences may also undergo spectacular eruptions, the prominence material being ejected into interplanetary space (see, e.g., Section 4.4 and references therein).

One feature discernible, e.g., in white light, radio, X-ray and visible emission coronal observations, is that of a region of lower brightness surrounding the prominence, usually referred to as a "cavity". The cavity phenomena above prominences have been observed since many years (see, e.g., Waldmeier, 1970). During the Skylab era, they began to be observed systematically. Among the great variety of different phenomena occurring in the inner solar corona however, the relationship between prominences (filaments) and cavities above them, is still an open question. Observations of the solar corona at different wavelengths suggest that the cavity can be interpreted as depletion in the electron density in the corona along the line of sight.

\section{An example: Morphological description}

Many of the green line images obtained with the MICA telescope show regions where the intensity is less than in the respective surroundings. These regions are observed to retain their shapes during the whole observing day, i.e., they are stationary regions characterized by intensity depletion (following the notation used in Section 4.2, $I_{S}<<I_{R}$ holds for these regions). They are observed to lie close to the limb, spanning several altitudes and angular extents at different position angles. When the weather at El Leoncito is such to permit observations during several consecutive days, these regions are observed to follow the rotation of the Sun.

In order to interpret correctly and characterize the nature of the emission of these darker regions, observations taken in $\mathrm{H}_{\alpha}$ emission with the HASTA telescope (Appendix C) are also used. As an example, we selected two images taken with both instruments on December 6, 1998. Figure 4.4 shows a composite of a $\mathrm{H}_{\alpha}$ image taken at 13:01 UT and a green line one obtained from an on-line image taken at 13:01:16 UT (the continuum used to treat the on-line image was taken at 13:01:33 UT). The most relevant features that can be observed are:

- At the south-eastern limb a green cavity (A) is seen to lie above a $\mathrm{H}_{\alpha}$ prominence (B).

- At the western limb, two bright $\mathrm{H}_{\alpha}$ features above the limb (one of the them labelled $\mathrm{C}$, the other too faint to be discerned in the picture) seem to be the footpoints of two dark channels (D1 and D2) which connect at higher altitudes (i.e., at $\sim 1.4 \mathrm{R}_{\odot}$ ). Images of HASTA corresponding to previous days show that these features are part of a disappearing filament.

- At about $25^{\circ} \mathrm{N}$ (western limb) we notice another prominence (E) with a cavity extending towards north (F).

- Having in mind projection effects, a dark cavity $(\mathrm{G})$ in the green line emission seems to extend above a $\mathrm{H}_{\alpha}$ filament close to the north pole $(\mathrm{H})$. 
- A highly structured prominence is seen at the NE limb (J) spanning $\sim 20$ degrees (between $\sim 20^{\circ} \mathrm{N}$ and $\sim 40^{\circ} \mathrm{N}$ apparent latitude). The dark coronal structure (I) above this prominence appears to be filled with the prominence threads.

\section{Characterization of the features observed}

The selected example is not an isolated one. As already mentioned, in most of the observations (not only on that day) a well defined cavity can be distinguished above either quiescent filaments close to the limb or prominences

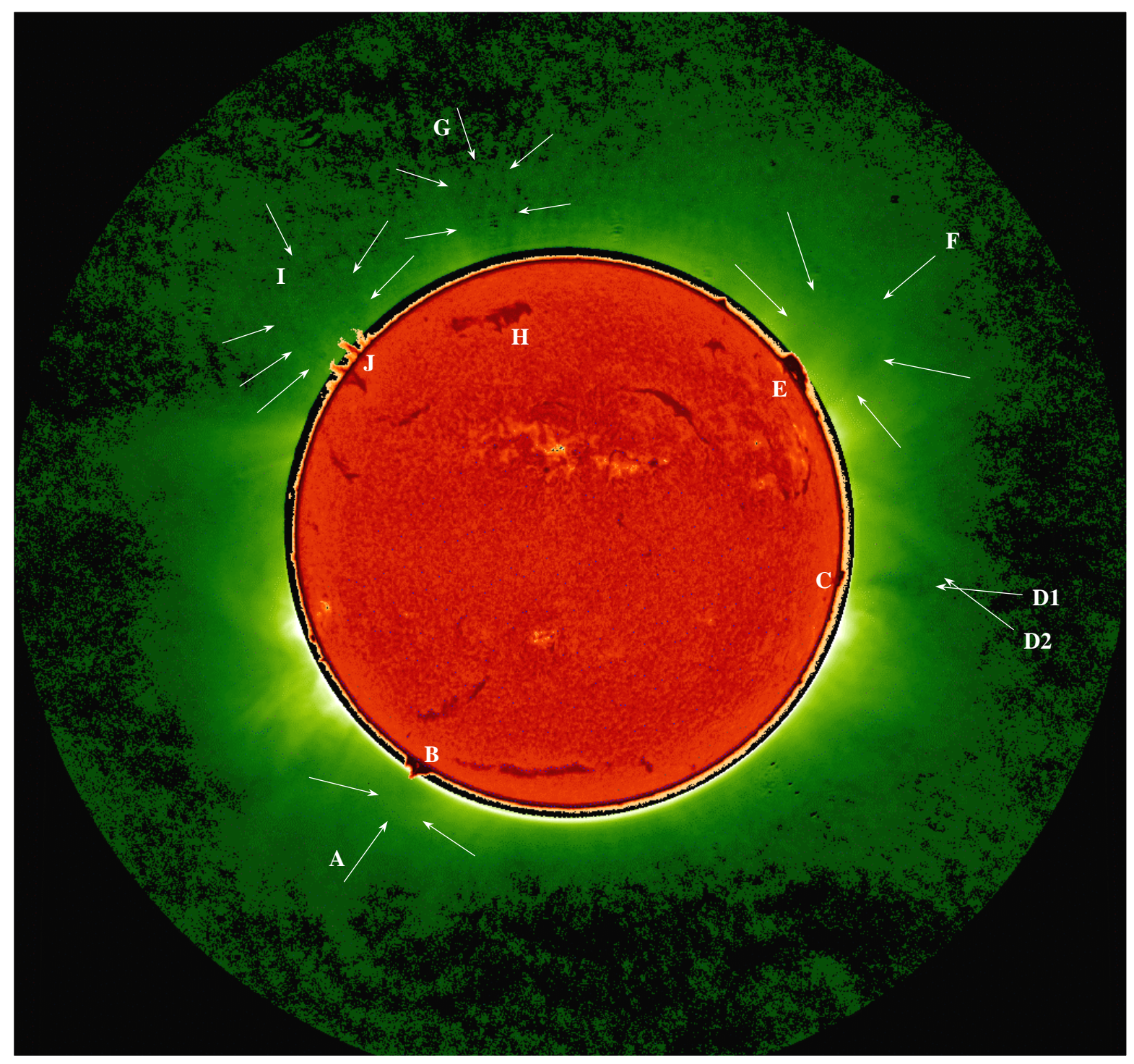

Figure 4.4: Composite of MICA (green) and HASTA (red) images on December 6, 1998. The HASTA image was taken at 13:01 UT with $0.5 \mathrm{msec}$ exposure time. The MICA image results from an on-line image taken at 13:01:16 UT (20 sec exposure time) and treated with a continuum image taken at 13:01:33 UT (4 sec exposure time). The northern limb is shown up, and the western limb at right. For details see text. 
(line of sight effects permitted).

Inspection of running differences images on that day reveals a scenario compatible with that of the stationary case for the whole MICA field of view. Therefore, the interpretation of coronal features must be done in the context of (4.5). Let us thus say a few words about the factors that can yield to observe the coronal features described above. Intensity depletion (i.e., $I_{S}<<I_{R}$, see Section 4.2) is observable in a "treated" coronal MICA image either because:

1. there was a temporary enhancement of the continuum intensity at the time of the off-line image used to treat the on-line one (i.e., $\varepsilon_{S}\left(\left|t_{i}-t_{j}\right|<0\right)$. This means, the electron density was temporarily enhanced or

2. there was a temporary decrease of the line intensity at the time of the on-line image. This could occur because the emitting region suffered either a temperature change or electron density decrease at the time of the on-line image.

Since the examples of "cavities" mentioned above persisted for the whole day as observed by MICA, a temporary change in intensity (either continuum or line intensity) must be discarded. Therefore, the temperature and electron density of the emitting plasma must be stationary. In this case, either

1. the region has a temperature far off the temperature range necessary to produce significant green line emission (see, e.g., Figure 4.1), and/or

2. the electron density is much less than in the surroundings.

Monochromatic observations of the region do not allow to discern between both possibilities. However, closer inspection of the off- and on-line images separately, would allow the distinction provided the intensity depletion in the region of interest is large enough to be distinguished from the surroundings.

\subsection{Coronal transients}

Dynamic features in the green line corona have been recorded with unprecedented sensitivity by the internally occulted C1 instrument of the Large-Angle Spectroscopic Coronagraph (LASCO) onboard the Solar and Heliospheric Observatory (SOHO) (Brueckner et al., 1995). The structural changes of the green line corona have been observed to be fairly gradual. This gradual evolution of the green line structures eventually leads to very different large-scale patterns (Schwenn et al., 1997). Further, in the short scale, the underlying active regions have been observed to produce sudden changes resulting in a complete rearrangement of the magnetic field pattern, many times giving origin to spectacular eruptions of matter from the Sun, usually known as coronal mass ejections (CME).

CMEs were first observed by coronagraphs from OSO-7 (Tousey, 1973) and Skylab (Gosling et al., 1974). The CMEs are huge ejections of gas clouds, often with masses $>10^{16} \mathrm{~g}$ and energies $>10^{32} \mathrm{ergs}$, and frequently subtending more than $60^{\circ}$ in position angle (see, e.g, Howard et al., 1985; Hundhausen, 1999). Further, they cover a wide range of speeds and very often are associated with interplanetary shocks (Hundhausen, 1972; Schwenn, 1986). Hundhausen (1984) defined a CME as "an observable change in coronal structure that i) occurs on a time scale between a few minutes and several hours and ii) involves the appearance of a new discrete bright light 
feature in the coronagraph field of view". This definition of CMEs has been very widely accepted to be the most appropriate one as it stresses on the observational aspect and the transient nature of the phenomena while eluding the interpretation of the feature itself and its potential origin.

There are two types of classification of CMEs based on observations by several past and present space coronagraphs. One classification is based on structure and morphology while the other is based on the kinematic properties of CMEs. From a study of a large number of CMEs recorded in white light by the SOLWIND/P78-1 coronagraph (Koomen et al., 1975), Howard et al. (1985) classified CMEs into various types according to their structure and morphology. These types of CMEs included loop, curved front, halo, spike, double spike and streamer blow-outs. Another class of CMEs was named as "complex", indicating that they did not have a well defined structure to fit into the other subclasses.

On the other hand, measurements of flow speeds and acceleration in CMEs are of utmost importance in understanding the initiation and propagation of CMEs. The speeds of CMEs are known to lie in the range 50$2000 \mathrm{~km} / \mathrm{sec}$. MacQueen \& Fisher (1983) classified CMEs based on the kinematics of a large number of coronal transients observed from both the space coronagraph on Skylab and a ground-based coronagraph. Under this classification, CMEs can be divided in the following two major classes:

- Eruptive or slow events:

They start with low speeds of the order of $10-20 \mathrm{~km} / \mathrm{sec}$ and attain values of acceleration which range between $0-50 \mathrm{~m} / \mathrm{sec}^{2}$.

- Flare associated or fast events:

They start with a sudden initial acceleration and high speeds $(>700 \mathrm{~km} / \mathrm{sec})$, and propagate at practically constant speed after the triggering.

The study of slow CME is of renewed interest, in context with the study of Sheeley et al. (1997). They identified in LASCO-C2/C3 images slowly moving density enhanced blobs or puffs which move in the ambient slow solar wind like "leaves in the wind" with slow speeds up to $300 \mathrm{~km} / \mathrm{sec}$ and may be considered as small-scale mass ejections. Srivastava et al. (1999) attempted to look for corresponding features of slowly moving blobs in the inner corona, i.e., in the field of view of LASCO-C1 (1.1-3.0 $\left.\mathrm{R}_{\odot}\right)$ but none of such small scale features could be detected in Fe XIV or Fe X emission line observations. Instead, a distinct class of slow CMEs which were relatively rare in occurrence could be identified. They are found to be structurally distinct and to evolve as low-lying loops for several hours in the $\mathrm{C} 1$ field of view, before crossing gradually the entire field of view of $\mathrm{C} 2$ and $\mathrm{C} 3$ coronagraphs in several hours (of the order of a day). The majority of them are associated with prominence eruptions. A bright leading edge, a dark cavity underneath, and a bright core inside the cavity usually related to cold prominence material are the typical signatures in the coronagraph field of view in this type of events. They are seen to originate close to the limb, at around $1.5 \mathrm{R}_{\odot}$ near the large-scale streamers and to change the nearby large-scale streamers. This kind of CME starts rising slowly with speeds $<100 \mathrm{~km} / \mathrm{sec}$, with majority even less than $50 \mathrm{~km} / \mathrm{sec}$ below $3 \mathrm{R}_{\odot}$. The terminal speed beyond $20 \mathrm{R}_{\odot}$ is close to that of the ambient slow solar wind (Srivastava et al., 1999). In the past, only a few observations of similar type of slow CME have been reported, e.g., by Fisher \& Garcia (1984), 
based on ground-based observations made with the MK-III K-coronameter within the range of 1.24 to $2.3 \mathrm{R} \odot$. Since the magnitude of the speeds of these slowly evolving balloon-type events is comparable to that of the slow solar wind as traced by the "leaves in the wind", it appears that gradual CMEs also drift away in the slow solar wind.

Sometimes, the sudden changes responsible for the initiation of such events could be well recorded with the available time resolution of the instruments. But at other times, these changes are so fast that they escape the field of view during the observation time gaps. It is thus of crucial importance that observations of such events at higher cadence close to the limb be recorded in order to reveal more precisely the onset and time evolution of such transients. An advantage of the ground-based telescopes such as MICA and HASTA over the space-based ones is that they can achieve a much higher time resolution because they are not constrained by telemetry. Another advantage of the MICA instrument is that it can observe lower parts of the corona $\left(1.05 \mathrm{R}_{\odot}\right)$ than LASCO-C1 $\left(1.1 \mathrm{R}_{\odot}\right)$, closer to where the core of the prominence lays and where flares likely originate.

The trigger mechanism of such transients and the nature of their acceleration as they propagate outward in the corona are some of the questions that remain unsolved (see, e.g. Forbes \& Priest, 1995; Magara \& Shibata, 1997). In particular, the question of the role of prominences or flares in triggering or driving the transients that give origin to CMEs has not been settled yet. Therefore, the morphological study of such events (which involve, e.g., eruptive prominences and flares), provides information about the magnetic topology of the loops involved, and allows the analysis of the trajectory of the material released.

Simultaneous observations at different wavelengths, i.e., different temperatures, provide an important clue to the understanding of the cause and development of such transients. In this regard, the observations recorded by the HASTA telescope (Appendix C) represent the ideal complement for the analysis of coronal transients. In particular, a comparative study allows to determine the spatial origin of the features that give rise to the coronal transients as well as discern between ejected and cooled down material, both of them characterized by intensity depletion in the coronagraph field of view.

Therefore, the morphological description of a fast and complex limb-coronal transient is addressed. The interpretation of the features observed in MICA images is guided by the mathematical framework outlined in Section 4.2. Images from the HASTA telescope are also used to help in the interpretation.

\subsubsection{The September 30, 1998 event}

\section{An overall view}

The observation time on September 30, 1998 in El Leoncito started at 11:00 UT and finished at 21:00 UT approximately. Several transients were observed to occur above the NOAA Active Region (AR) 8340 during a time lapse of around 4 hours, close to the western limb at around $20^{\circ}-23^{\circ} \mathrm{N}$ latitude. This AR included an extended plage area surrounding a big spot, and at least three long filaments, which had been observed by HASTA in previous days (see Figure 4.5).

On September 30, a subflare and a $2 \mathrm{~N} \mathrm{H}_{\alpha}$ flare occurred in the AR 8340. The $\mathrm{H}_{\alpha}$ subflare was reported to 


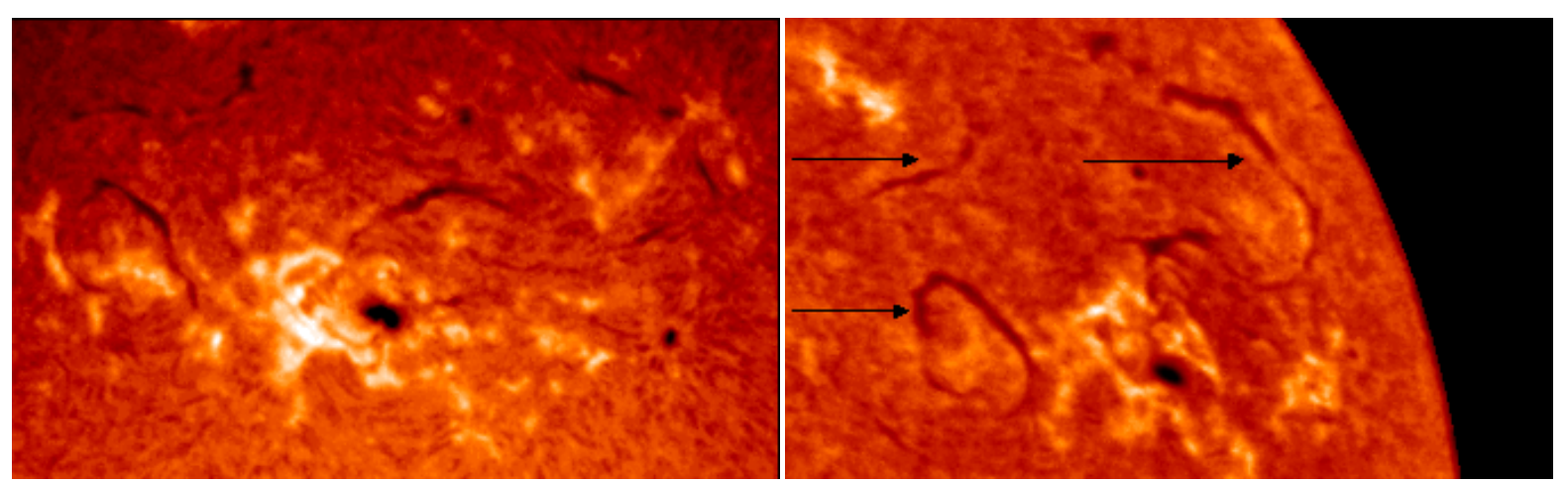

Figure 4.5: Partial field of view of HASTA on September 23 (left) and September 27, 1998 (right) for the AR 8340. The three arrows (right panel) show the position of three long filaments (for details see text). North is at top, west at right.

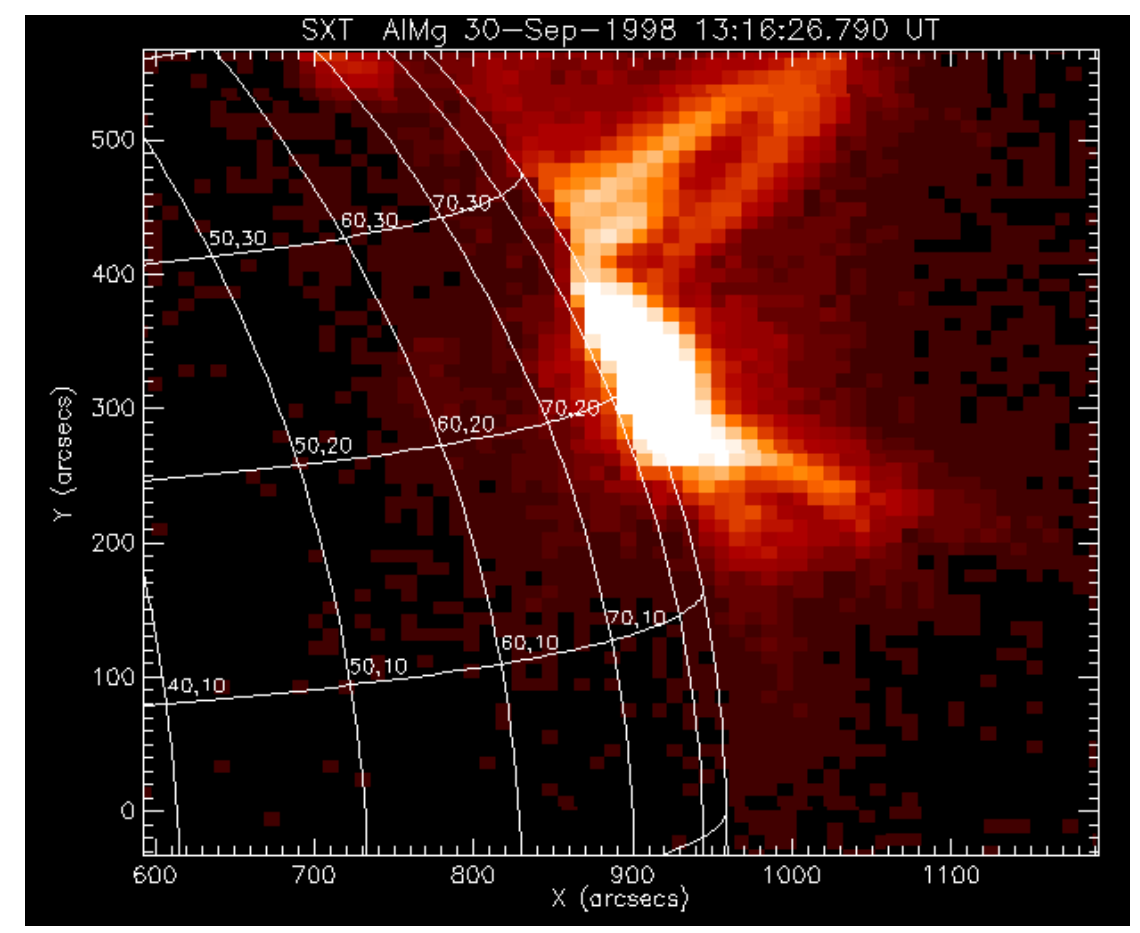

Figure 4.6: $S X T$ YOHKOH image of the subflare which occurred above the NOAA AR 8340 on September 30, 1998 at $\sim 13: 16$ UT.

start at 13:14 UT and the $2 \mathrm{~N} \mathrm{H}_{\alpha}$ flare at 14:02 UT, reaching their maximum intensity at 13:31 UT and 14:34 UT, respectively ${ }^{8}$. At the time of the subflare, i.e, at $\sim 13: 16 \mathrm{UT}$, an enhancement in soft X-ray with structure similar to that of a jet was recorded by SXT/YOHKOH (Tsuneta et al., 1991), as can be seen in Figure 4.6. A similar enhancement was also seen by both HASTA and MICA instruments in the $\mathrm{H}_{\alpha}$ and Fe XIV (green line) emission,

\footnotetext{
${ }^{8}$ As reported in the Solar Geophysical Data, October 1998, Number 650, Part 1.
} 


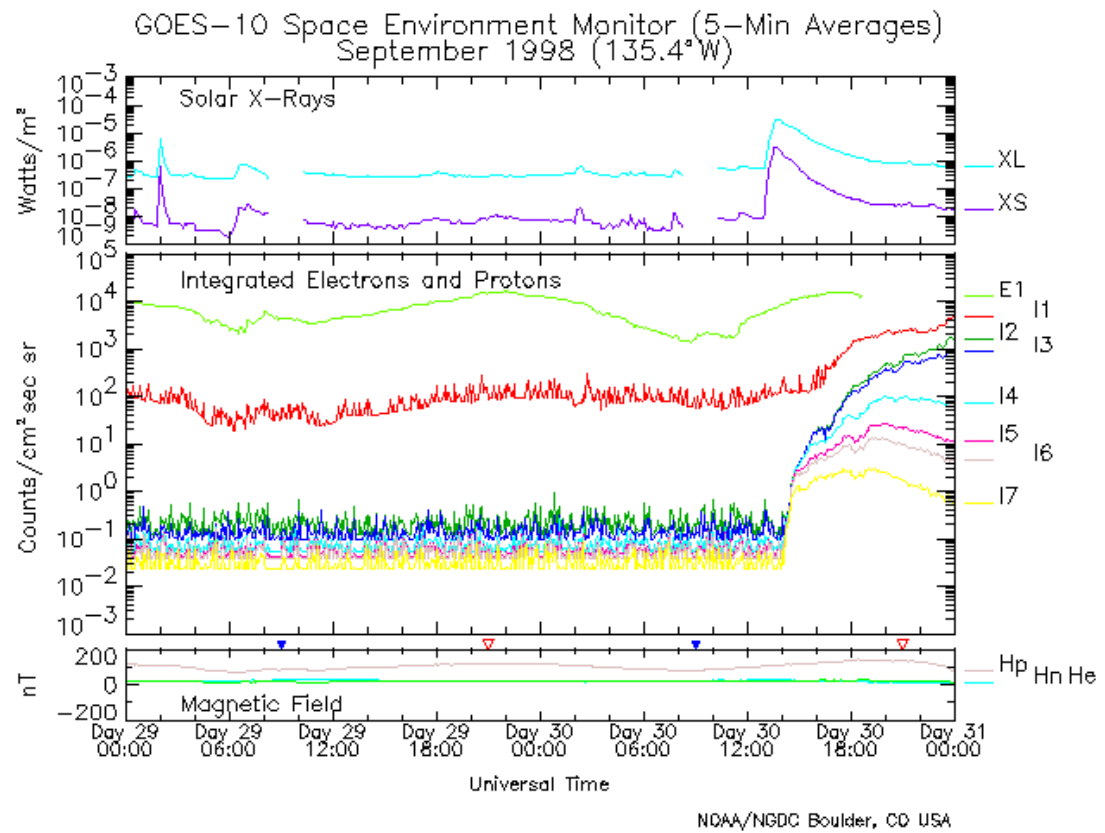

Figure 4.7: GOES data for the September 30, 1998 event. Solar X-rays: XL: 1-8 $\AA$ (Long X-rays); XS: .5-4 (Short $X$-rays). Energetic Particle Channels (E: electrons; I: protons): E1: > $2 \mathrm{MeV}$; I1: >1 MeV; I2: > $5 \mathrm{MeV}$; I3: > $10 \mathrm{MeV}$; I4: $>30 \mathrm{MeV}$; I5: > $50 \mathrm{MeV}$; I6: > $60 \mathrm{MeV}$; I7: > $100 \mathrm{MeV}$. Magnetic filed components: Hp : Parallel to satellite spin axis, or northward; He: Earthward; Hn: Normal to Hp and He (points East).

respectively. After the green line jet ${ }^{9}$, i.e., at $\sim 13: 25 \mathrm{UT}$, more release of material in form of blobs ${ }^{10}$ was also recorded by both instruments. An enhancement in the X-ray emission has also been detected by instruments onboard the Geostationary Operational Environmental Satellite GOES-10. Further, a sudden increase in energetic particles (i.e., protons with energies above $5 \mathrm{MeV}$ ) arriving at Earth and being likely correlated with the event was also detected by the GOES-10 satellite (see Figure 4.7). Finally, during the development of the $2 \mathrm{~N} \mathrm{H}_{\alpha}$ flare (more precisely between 14:20 UT and 14:35 UT), HASTA observed the eruption of a prominence. No noticeable changes were observed at this time in the green line emission by MICA.

\section{The Jet}

Figure 4.8 shows the global time development of the event as recorded by MICA combined with HASTA observations, for the time lapse between 13:15 UT and 13:45 UT. Unfortunately, the HASTA telescope was working on that day only with a cadence of 1 image every 5 minutes. The beginning of the event at coronal altitudes was observed by MICA at 13:15:45 UT. At this time, a small dark dot appeared, just at the border of the inner edge of the MICA field of view at around $20^{\circ} \mathrm{N}$ apparent latitude (Figure 4.9 a). This dark dot is seen to fade in the next

\footnotetext{
${ }^{9}$ We call the observed transitory green line emission enhancement (with apparent collimated motion) a "green line jet" in analogy to the findings made by Shibata et al. (1992) and Strong et al. (1992) based on YOHKOH observations.

${ }^{10}$ Blobs: bright enhancements seen in the field of view of the instruments (above the limb).
} 

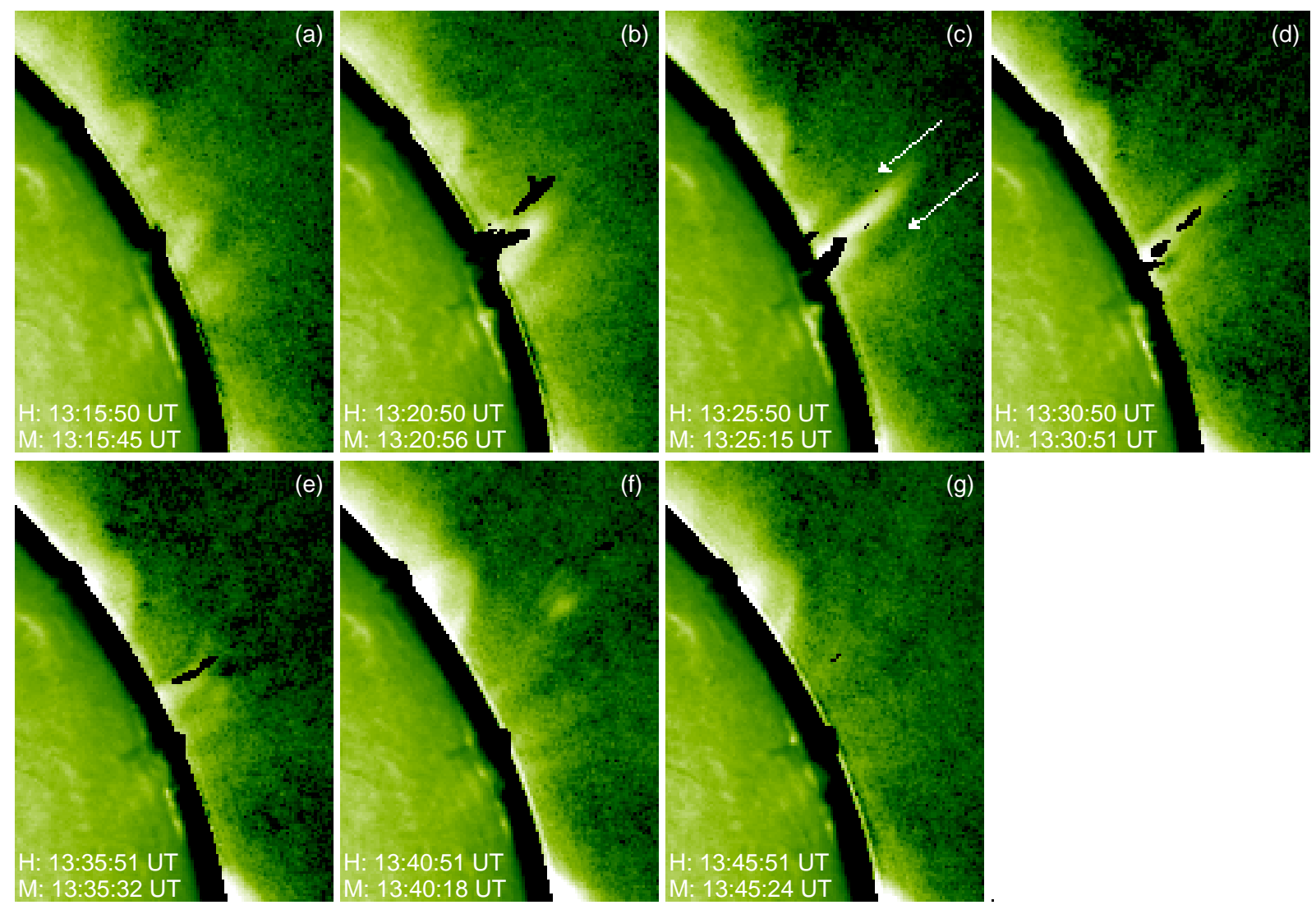

Figure 4.8: Combined observations of HASTA (H) and MICA (M) on September 30, 1998. MICA images are corrected according to the calibration procedure described in Chapter 3. The $H_{\alpha}$ emission above the limb is shown in black for better contrast.

two images, which were taken at 13:16:13 UT and 13:16:44 UT (Figure 4.9, frames b and c). At 13:17:50 UT, the dark dot cannot be distinguished any longer ${ }^{11}$.

At the time of the appearance of the dark dot in MICA images, i.e., 13:15:45 UT, the $\mathrm{H}_{\alpha}$ images of HASTA show a small bright enhancement at the same apparent position of the dark dot. This enhancement in $\mathrm{H}_{\alpha}$ is related to a $\mathrm{H}_{\alpha}$ subflare reported to start at 13:14 UT in Solar Geophysical Data (Number 650, Part I, 1998). The next available $\mathrm{H}_{\alpha}$ image (at $\sim 13: 20 \mathrm{UT}$, see Figure 4.8 b) shows two elongated bright enhancements above the limb. Both of them likely correspond to the evolution of the previously recorded $\mathrm{H}_{\alpha}$ enhancement. At that time, i.e., 13:20 UT, two dark threads in the Fe XIV emission become visible, one of them (the northest one) being located above the original position of the $\mathrm{H}_{\alpha}$ enhancement. This dark thread is spatially coincident with the northern elongated $\mathrm{H}_{\alpha}$ enhancement, while the other one lies above another $\mathrm{H}_{\alpha}$ enhancement, as can be seen in Figure $4.8 \mathrm{~b}$. Further, as the contrast between the dark threads and the surrounding corona becomes more pronounced, eruption of material in form of a jet begins to be observed in the MICA images. The green jet is ejected at the same apparent

\footnotetext{
${ }^{11}$ In order to give the correct interpretation to this feature, it is important to note that the first three MICA images mentioned (Figure 4.9) were treated with a continuum taken at 13:17:11 UT while the latter with a continuum obtained at 13:18:13 UT. This fact strongly suggests that a gradual increase in the continuum intensity occurred in the time lapse between 13:15:45 UT and 13:18:13 UT.
} 

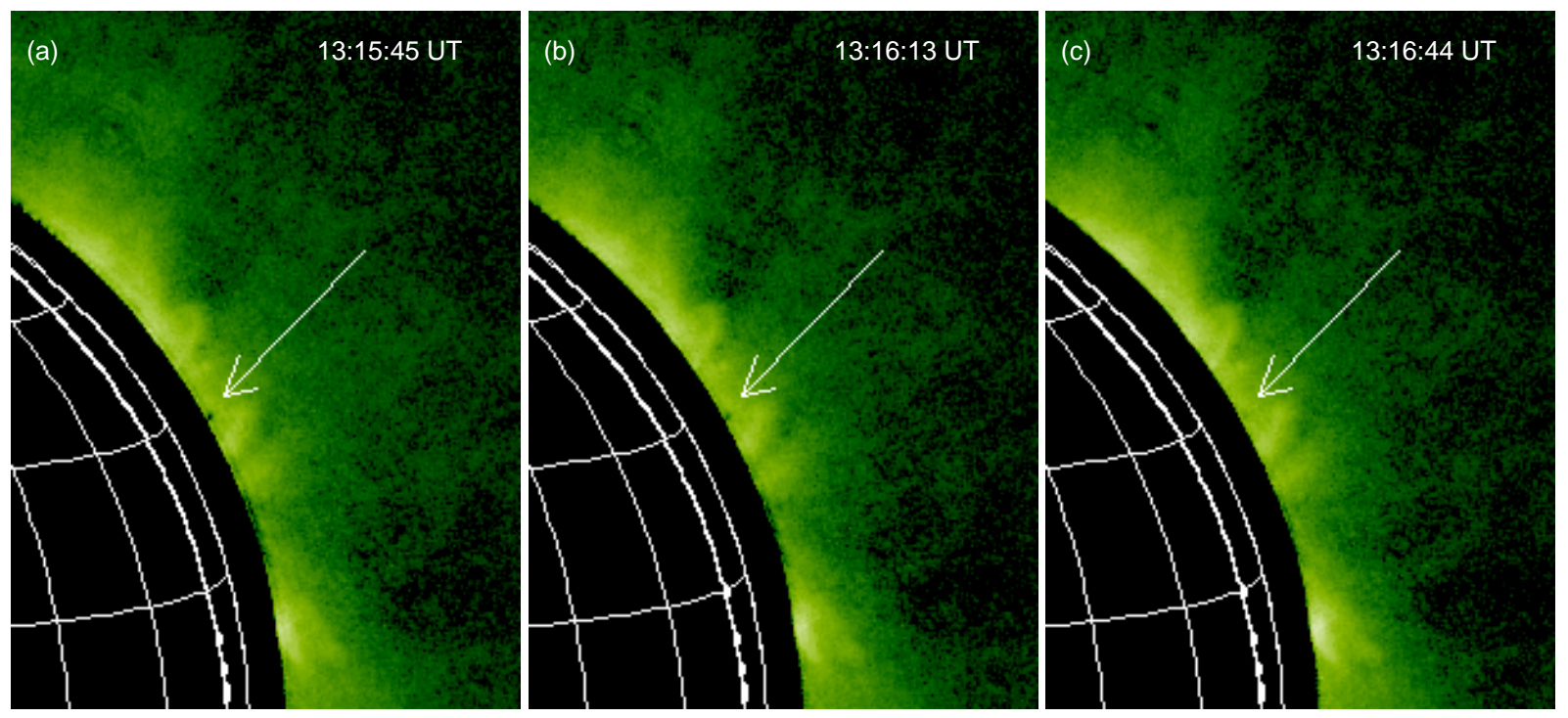

Figure 4.9: Beginning of the event on September 30, 1998 as recorded by MICA. The fading of the dark dot suggests a gradual increase of the continuum intensity. For details see text.

position of the southern elongated $\mathrm{H}_{\alpha}$ enhancement. This spatial coincidence can be noted in Figure $4.8 \mathrm{c}$.

It may be conjectured that the two dark threads seen in MICA images (shown by arrows in Figure $4.8 \mathrm{c}$ ) are likely the two legs of a dark loop, which begins to rise simultaneously with the jet (lack of contrast in MICA images does not allow us to confirm it). The green jet moves outwards almost radially, in a trajectory having little or no curvature. Its duration was around 11 minutes until the maximum elongation was reached. The outermost part reached a projected heliocentric distance of $\sim 1.24 \mathrm{R}_{\odot}$. As said above, the jet is seen split in two in $\mathrm{H}_{\alpha}$ emission, one of the arms being coincident with the green jet, and the other one with the northern leg of the "dark loop". The latter is observed to disappear in the $\mathrm{H}_{\alpha}$ image taken at $\sim 13: 25$ UT, while the one coincident with the green jet remains (Figure $4.8 \mathrm{c}$ ).

Inspection of HASTA images as a movie suggests that the $\mathrm{H}_{\alpha}$ jet spatially coincident with the green line one, is ejected at approximately the same projected velocity as the green line jet $(\sim 250 \mathrm{~km} / \mathrm{sec})$. At around 13:37 UT the green line jet is no longer visible. After its disappearance, the release of a blob is observed in MICA images. This blob is first seen at the original position of the southern leg of the "dark loop", i.e., displaced from the original position of the green jet (figures $4.8 \mathrm{e}$ and f, and Figure 4.10). Note in Figure 4.10, frames a to c, the existence of a dark spot (marked with an arrow pointing down). The height-time diagram of both the green jet and blob is shown in Figure 4.11. Simultaneously, release of very faint blobs could also be seen in HASTA images.

Before the ejection of these plasmoids, another dark dot was observed in MICA data (see Figure 4.12). It first appears at 13:27:51 UT, slightly southwards from the first one, just between the green line jet and the southern leg of the "dark loop". As before, it is again detected in the next image (at 13:28:28 UT), this time with less contrast. At 13:28:58 UT it is completely gone ${ }^{12}$.

\footnotetext{
${ }^{12}$ The three images mentioned were treated with the same continuum, obtained at 13:29:15 UT. Also in this case, the observational evidence suggests the occurrence of a gradual increase of the continuum intensity.
} 
Since the beginning of the event as observed by MICA, a gradual change in the global structure of the green line emission at large scale is also seen. At the time of the occurrence of the first dark dot this change no longer remains gradual but becomes more abrupt. The removal of hot and cold material can be recognized better when viewing the MICA and HASTA images as a movie. This global change was observed in MICA images until around 14:30 UT on September 30, 1998.

\section{Discussion}

The interpretation of this complex event can be guided by the dark dots observed in MICA images (at 13:15 UT and $\sim 13: 27$ UT). Their presence is due in both cases to a temporary enhancement of the continuum emission, as revealed by the MICA continuum images. Since the continuum emission is sensitive to the Thomson scattering of the photospheric light by free electrons in the corona, the intensity change only reflects excess mass. These dots are located at the footpoints of the "dark loop" observed in MICA images. The first one could be seen close to the northern footpoint of the loop, and the latter near the southern one. Furthermore, the second one appears to rise, following a trajectory coincident with the southern leg of the "dark loop". The first electron density increase is observed at around the same starting time (and position) of the $\mathrm{H}_{\alpha}$ subflare, and could be interpreted as evidence of magnetic reconnection at the point where the dark dot appears. The release of material in the form of a jet seen in emission both in $\mathrm{H}_{\alpha}$ and in the green line at the same apparent location and time shows that this material spans a wide range of temperatures. Magnetic reconnection could therefore be the possible mechanism which heats the relative cold material to temperatures approaching $1.8 \times 10^{6} \mathrm{~K}$ (since only at these temperatures the structures become visible in the green line) and triggers the jet.

On the contrary, the second part of the jet, seen in emission in $\mathrm{H}_{\alpha}$ but at the location of the "dark loop" in the green line, shows that this material is colder and more massive than the other one. When the second dark dot appeared at the southern leg of the "dark loop", cold material was still being released, as observed in HASTA images. In this case, the temperature was not high enough to be observed by MICA. However, after the appearance of the second dark dot, a green blob could be seen rising towards a dark spot. The existence of the dark spot
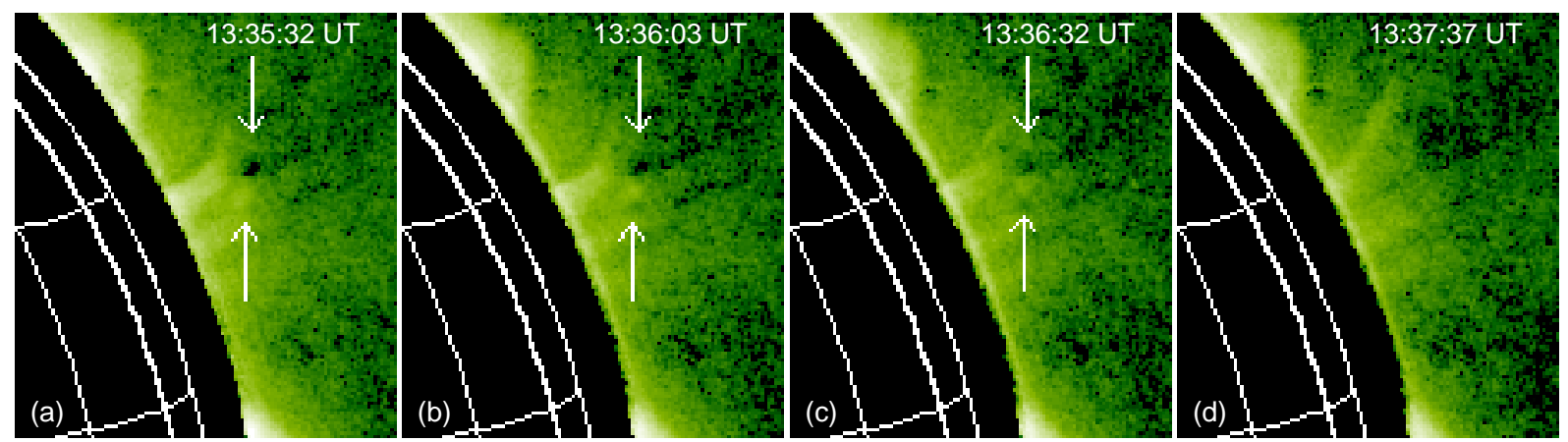

Figure 4.10: Blob release. The on-line images corresponding to panels (a), (b), and (c) were treated with a continuum image taken at 13:37:00 UT, while the one in panel (d) with a continuum taken at 13:37:56 UT. The movement of the bright blob towards the dark spot suggests that the bright blob has a strong continuum contribution, i.e., electron density excess. 


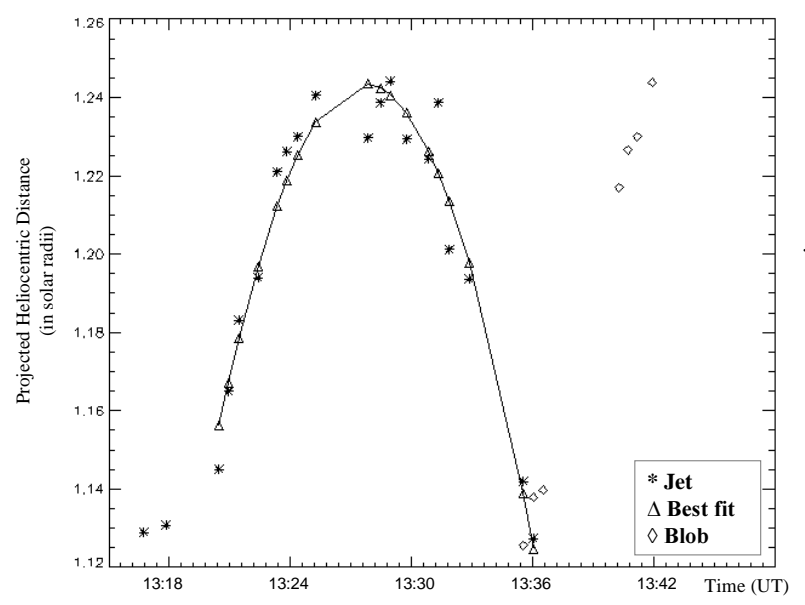

Figure 4.11: Tracking measurements of both the top of the jet and bright blob observed in MICA green line images on September 30, 1998, showing their height-time profile. The average projected speed for the rising phase of the jet is $\sim 250 \mathrm{~km} / \mathrm{sec}$ (as estimated from the "best fit", i.e., a least square quadratic fit), similar to that of the blob.

(Figure 4.10, frames a to c) strongly suggests that the analysis and interpretation of this moving bright green blob MUST BE done in the context of the nonstationary case outlined in Section 4.2. The analysis thus suggests that the bright blob is due to a continuum enhancement rather than a green line enhancement. This fact confirms the cold temperatures of the material ejected despite the fact of being observed in the green line images.

Thus, as the green line emissions traces the magnetic field topology (Schwenn et al., 1997), it seems likely that a big disruption of the magnetic field had taken place during this time, possibly giving rise to a CME. This fact is supported by the magnetic complexity of the region (see, e.g., Figure 4.5), a feature that has long been known to be a necessary condition to major eruptive activity (Patty \& Hagyard, 1986). Thus, the gradual change observed at
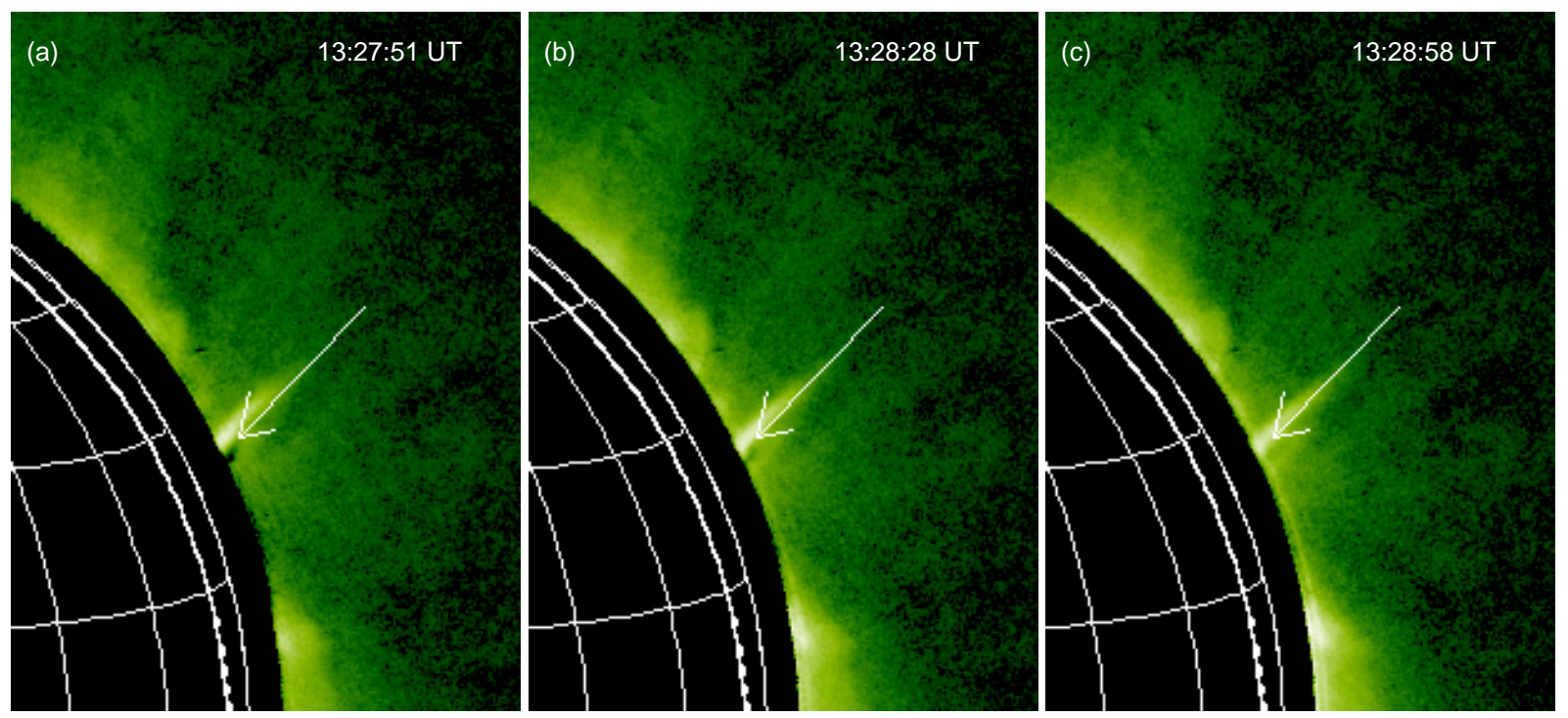

Figure 4.12: Appearance and fading of the second dark dot observed by MICA. As in the case shown in Figure 4.9, the fading of the dark dot suggests a gradual increase of the continuum intensity, i.e., a gradual increase of the electron density. For details see text. 
large scales would be a signature of the evolution of a gradual CME as it travel outwards.

\subsection{Concluding remarks}

One of the most interesting and key questions that can be addressed with MICA is that of the origin and evolution of coronal transients “very close to the limb". The excellent straylight and vignetting properties of the MICA telescope near $1.05 \mathrm{R}_{\odot}$ permit observations of the early evolution of expansion of classical coronal loop transients. Further, its relative high time resolution (only $\sim 3 \mathrm{sec}$ time gap between on-line images) makes the MICA instrument, the ideal tool for the i) detection of coronal transients, ii) study of their evolution, and iii) determination of their onset time, in particular, of those of explosive nature. For a further example of the MICA contribution to such studies the reader is referred to Innes et al. (2000).

However, as instrumental constraints do not allow taking the off-line images simultaneously with the on-line ones, the model for the continuum emission we use does not correspond exactly to the continuum emission at the time of the on-line observation we want to correct. Therefore, rapid changes in the continuum emission can lead to misinterpretation of the observations. In this respect, for the correct interpretation of the features seen in MICA images (especially in cases of dynamical events), it is of fundamental importance to follow the analysis outlined in Section 4.2, as demonstrated by the existence of the dark dots and moving bright blobs in the September 30, 1998 event. The interpretation of these features presents, in addition, difficulties inherent to observing in an optically thin medium. Since one observes integrated intensities along the line of sight throughout the coronal atmosphere, electron density and temperature intensities can be detected but not, in general, precisely located. Further, during the triggering and developing of explosive events, large line of sight velocities may move part of the associated CME outside the passband of the filter (because of the great Doppler shifts), making the interpretation of this kind of phenomena extremely difficult.

Thus, for any particular study using MICA images, it is very important to bear in mind that the interpretation of images be carried out keeping in view the aforementioned constraints of the instrument and imaging technique, as has been outlined in this Chapter. 



\section{Chapter 5}

\section{On the Rotation of the Emission Solar Corona}

In the previous Chapter, interpretation and analysis of coronal features at short time scales (minutes to hours) was performed. Now, we extend the analysis to longer time scales in order to study the rotation rate of the emission solar corona. The weather conditions and the day-night time cycle prevent MICA from producing a sufficiently homogeneous and continuous data set. Therefore, data from instruments on board the SOHO spacecraft were used for such a study. Its vantage position at the L1 Lagrangian point ${ }^{1}$ allows data to be acquired continuously.

Many studies have shown that small-scale short lived structures exhibit differential rotation associated to one of their footpoints in the photosphere. In contrast, the large-scale corona (be it in white light or the green coronal line) shows an almost rigid rotation at the equatorial rate. Ulysses in-situ measurements of solar wind speed, density and magnetic field strength confirmed this rotation rate even for very high latitudes but showed that near the streamer belt periodicities of longer periods are also present.

For studying this apparent discrepancy, we analyze first LASCO-C1/SOHO (Brueckner et al., 1995) data from April to October 1996 in order to investigate the dependence with latitude and solar distance of the periodicity and recurrence of Fe XIV emission structures. Secondly, we investigate the latitudinal dependence of the solar rotation rate by studying the longitude displacement of transition region and coronal features in their passage through the central meridian. For this study we used images obtained by the Extreme-Ultraviolet Imaging Telescope EIT/SOHO (Delaboudinière et al., 1995) in four wavelengths. The emission at those different wavelengths is representative of different heights in the solar atmosphere. Comparison of the obtained rotation profiles with the well known differential rotation rate of the photosphere is made, in order to better understand the behaviour of the rotation of the Sun and its atmosphere.

\subsection{Introduction}

Helioseismology studies (Kosovichev et al., 1997, and references therein) have demonstrated that the rotation of the interior of the Sun depends on latitude and depth. In the radiative core, e.g., the rotation is found to be almost rigid, while in the convection zone the transport of angular momentum by convective motions leads to differential rotation. On the other hand, various methods have been applied to determine the rotation rate of the Sun at photospheric levels, for example:

1. observations of Doppler shifts of photospheric Fraunhofer lines (Howard et al., 1983),

\footnotetext{
${ }^{1}$ In a system of two massive bodies revolving about each other (Sun-Earth or Earth-Moon), the Lagrangian points are the points where a third, much smaller body keeps the same position relative to the other two. They were named after French astronomer Louis Lagrange (17361813) who first studied them and who showed that there exist five such points in the Sun-Earth system. In the Sun-Earth system only two are important, both on the Earth-Sun line, i.e., the L1 point at 236 Earth radii sunward of Earth, and the L2 point at a similar distance on the night side. The L1 point is a good "early warning" outpost intercepting shocks and particles emitted by the Sun.
} 
2. tracking of Doppler features (supergranules) in the photosphere (Snodgrass \& Ulrich, 1990),

3. observations of the longitude displacement of magnetic features after a given time interval (Snodgrass, 1983),

4. determination of the recurrence of the magnetic field pattern at the central meridian after an integer number of solar rotations (Stenflo, 1989),

5. tracking of visible features such as sunspots, etc. In fact, sunspots have been used as tracers for solar rotation from the time they were first recognized as features on the Sun. Numerous studies of solar rotation can be found in the literature dating from Scheiner (1630), through Carrington (1863), Newton \& Nunn (1951), to the recent studies of Howard et al. (1984), Gilman \& Howard (1984, 1985), and Ternullo (1990).

It is interesting to note that, depending on the method used, different patterns of differential rotation are obtained. By determining the longitude displacement of magnetic features, Snodgrass (1983) found a differential rotation pattern which (except for being about $2 \%$ less pronounced close to the poles) is in good agreement with that obtained by Howard et al. (1983) using spectroscopic methods. In contrast, when analyzing the recurrence of the magnetic pattern during several solar rotations (Stenflo, 1989), the rotation is found to be quasi-rigid, with a period of 29-30 days at 50-55 degrees and decreasing again at higher latitudes. There have been several explanations to such discrepancies. Stenflo (1989) suggested that the source regions of magnetic flux (long-lived) are likely located near the bottom of the convection zone with its almost depth-invariant angular velocity. More recent calculations based on random walk simulations made by Wang \& Sheeley (1994) have demonstrated how the rotation of the photospheric field may be quasi-rigid on global scales and yet strongly differential on smaller spatial scales.

Then, the question arises, how the rotation pattern measured at photospheric levels is conveyed to coronal heights. Intuition suggests that the inner solar corona should rotate synchronously with the underlying photosphere. EUV, $x$-ray, and visible observations of the inner emission corona show that magnetic fields penetrate into the lower regions of the solar corona with sufficient strength to drag the coronal plasma ${ }^{2}$. Naturally, a point must be reached further out where the corona is incapable to maintain this synchronous rotation pattern (see, e.g., Wang et al., 1988, and references therein).

The determination of the coronal rotation rate is based either in the study of the recurrence pattern of coronal features or on the tracking of the apparent motion of coronal features. Waldmeier (1950) and Trellis (1957) by studying the recurrent passage of bright green emission regions $(530.3 \mathrm{~nm})$ over the solar limb determined the rotation rate as function of latitude. Their results suggest that the corona below $1.25 \mathrm{R} \odot$ rotates with the underlying magnetic fields as revealed by filaments, i.e., differentially. On the other hand, the results obtained following a long-lived high latitude green emission region at $65^{\circ}$ latitude by Cooper \& Billings (1962) require the corona to rotate faster than the surface. Comparison of data of coronal and surface features gathered during different phases of the solar cycle may well yield misleading results. In a series of papers, Antonucci \& Dodero $(1974,1979)$ found that a global scale long-lived component of the green line corona (of the order of a year) rotates almost rigidly at the equatorial rotation rate independently of the phase of the solar cycle. Simultaneously, differential rotation is

\footnotetext{
${ }^{2}$ In a low $\beta$ plasma, as that of the inner solar corona, the plasma is "frozen" to the magnetic field.
} 
still observable in those latitude zones where activity is present (Antonucci \& Svalgaard, 1974). The coexistence of a global rotating component and a local differential one represents one of the most puzzling problems in the interpretation of coronal data. To this respect, Antonucci \& Dodero (1979) found that the short-lived green emission line features (less than 27 days), which are associated with stronger magnetic fields, do not disrupt the long-lived rigid rotating coronal pattern. Moreover, Roberts \& Goldstein (1998) have shown by using low frequency spectra of the wind speed, density, and magnetic field strength as measured by Ulysses, that near the streamer belt the solar wind at solar minimum exhibits many harmonics of fundamental frequencies corresponding to 26- and 34-days periods (sidereal). They showed that signatures of the photospheric differential rotation are detectable in-situ even at several astronomical units. They infer then, that the solar photosphere matters in producing the solar wind.

On the other hand, by using autocorrelation analysis, Sime et al. (1989) found that for the Fe XIV corona, the rotation curves progressed from a strongly differential profile in the ascending phase of the solar cycle to a quasi-rigid profile in the descending phase. In a more recent work (Inhester et al., 1999, hereafter Paper I), by analyzing 1 year of LASCO-C1 (Brueckner et al., 1995) data obtained around the minimum of the solar activity cycle, we have obtained results at global scales similar to those obtained by Sime et al. (1989). Nevertheless, the periodograms obtained with the method used in Paper I show on smaller spatial scales certain "peculiarities". Since the corona dictates the characteristics of the entire heliosphere, the study of the rotational characteristics of the inner corona might thus serve as an important tool for linking the photosphere and the inner heliosphere. In particular, crucial questions can be addressed as, e.g., whether the photosphere or deeper layers in the Sun matter in the processes producing and structuring the solar wind.

\subsection{Part I: Analysis of the recurrence of the green line emission}

The first part of the present Chapter is dedicated to review the method used in Paper I and interpret the "peculiar features" seen in the periodograms. Further, their possible relation with the rotation characteristics of the green line emission corona and its implications is outlined.

\subsubsection{The LASCO-C1 observations}

The well known green coronal emission line at $530.3 \mathrm{~nm}$ is due to a forbidden transition of the $\mathrm{Fe}^{13+}$ ions, which peaks at a temperature of about $1.8 \times 10^{6} \mathrm{~K}$ (Burgess \& Seaton, 1964; Arnaud \& Rothenflug, 1985; Esser et al., 1995). The high quality of LASCO-C1 data allows us to investigate the variation of the rotation rate of the green emission line corona with good spatial resolution in the range from 1.1 to 3.0 solar radii. For the present analysis we have scanned through more than 3000 flat-fielded and bias-corrected green line images taken between May 1996 and October 1996. White light observations from LASCO-C2 and C3 show that at this minimum phase of the solar cycle there is always a near equatorial "streamer sheet" surrounding the Sun. Furthermore, LASCO-C1 data show bright and apparently closed loop systems, almost permanently present, centered at apparent latitudes of $30^{\circ}$ to $50^{\circ}$ in both hemispheres (see, e.g., Figure 4.2). Their helmet-like extensions are bent towards the equatorial plane. The centers of these mid-latitude loops are usually found at the positions of magnetic neutral lines in photospheric 
magnetograms (Schwenn et al., 1997).

In this work we restrict the analysis of the LASCO-C1 green line images from 1.1 to 2.2 solar radii, where the signal to noise ratio is high enough to obtain a reliable period determination.

\section{The Time Series}

The re-appearance of persistent coronal features was used to map the coronal rotation. In order to build the time series, the images were rebinned using a polar coordinate system rather than the rectangular pixel pattern (see Figure 5.1), i.e.:

1. Radial rays were traced on each image, the angular space between them being $m^{\circ}$.

2. Each image was then divided in solar-centric annuli $\left(n \mathrm{R}_{\odot}\right.$ wide),

3. We call the resulting sectors extending $n \mathrm{R}_{\odot}$ in radial direction and $m^{\circ}$ in polar angle "superpixels". The new intensity value of each "superpixel" results from averaging the intensity values of all original pixels inside the respective "superpixel".

Then, a "cube" of intensities $I(r, P, t)$ is made out of the resulting binned images, the axis being $r$ (radial distance), $P$ (polar angle), and $t$ (time). Therefore, each point of the cube is representative of the intensity of the corona at the position $r, \varphi, t$. For each pair of values $(r, P)$ a 1-D time series is considered.

The resolution with which we analyze the coronal rotation depends on the radial and angular size of the sectors defined. For the present work, we restrict the analysis to the following spatial extent of the sectors: $n=0.055 \mathrm{R} \odot$ and $m=3^{\circ}$ (for comparison, $n=0.1 \mathrm{R}_{\odot}$ and $m=5^{\circ}$ were chosen in Paper I).

At first, data from the east and west limb are considered independent (method $\left.\mathrm{B}_{0}\right)$. However, as the Sun rotates, the same coronal plasma volume is observed on the opposite limb after half a rotation period. Therefore, data from

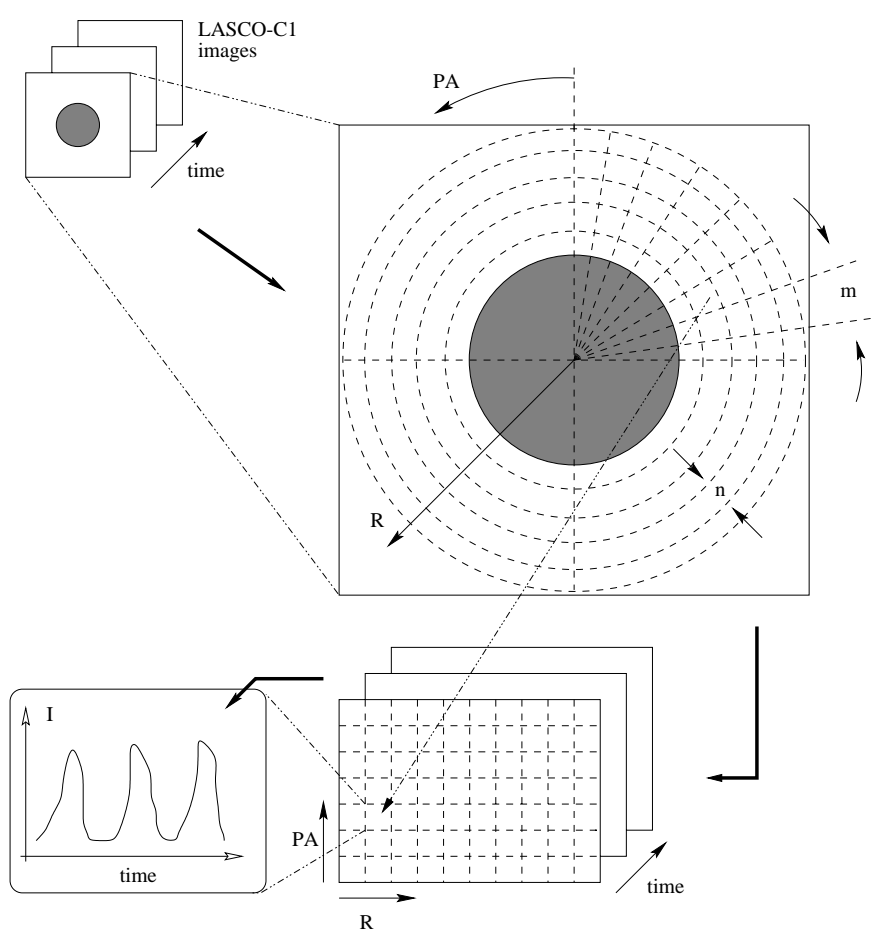

Figure 5.1: Sketch showing the building of the time series used to determine the recurrence of the green line intensity pattern. For details see text. 
opposite limbs and from the same latitude and radius can be expected to be correlated. Hence, in an alternative analysis (method $B_{1}$ ), we combine data from both limbs with the same latitude and radius by including a $180^{\circ}$ phase shift for data of the opposite limb. In order to reduce the noise level and get rid of wrong values that could alter the stationary behaviour of the time series, a smoothing technique based on the wavelet transform was applied to the time series. This technique of noise suppression employs the so-called à trous algorithm. For details of the method the reader is referred to Starck et al., 1997 and references therein.

Some minor data gaps are present in the observations, mainly due to periods of special spacecraft manoeuvres. Furthermore, the rate of image acquisition is not constant. In view of these facts, a method that deals with unevenly spaced time series is required in order to avoid artifacts which can arise from interpolation and/or zero filling.

\subsubsection{The Phase Dispersion Minimization technique: a brief overview}

As in Paper I, we used the Phase Dispersion Minimization (PDM) method (Stellingwerf, 1978), which is well suited to small and randomly spaced samples, and has no preference for a particular shape (e.g., sinusoidal) of the curve. Briefly, it evaluates the scatter of the data about a derived mean curve as a function of a trial phasing frequency (period), the frequency (period) producing the least possible scatter about the derived curve being chosen.

In the simplest version of this method, the overall rms scatter $\sigma$ of the measured emission intensity $e(t)$ in each of the radius and latitude bins mentioned above is derived in the following way. First, we determine

$$
\left\{\begin{array}{c}
N \\
E \\
\sigma
\end{array}\right\}=\sum_{t}\left\{\begin{array}{c}
1 \\
e(t) / N \\
(e(t)-E)^{2} /(N-1)
\end{array}\right\}
$$

where $N, E$, and $\sigma$ are the number of data points, their average, and rms scatter, respectively. Next, for an assumed period $T$, similar values are calculated for all data points that fall into one of $M$ temporal phase bins defined by the integer fraction of $t / T$. This yields similar values as above for each of the $M$ phase bins:

$$
\left\{\begin{array}{c}
N_{i} \\
E_{i} \\
\sigma_{i}
\end{array}\right\}=\sum_{t \mid M \operatorname{frac}\left(\frac{t}{T}\right) \in\left[1-\frac{1}{2}, i+\frac{1}{2}\right]}\left\{\begin{array}{c}
1 \\
e(t) / N_{i} \\
\left(e(t)-E_{i}\right)^{2} /\left(N_{i}-1\right)
\end{array}\right\}
$$

If the right period $T \approx T_{\text {rot }}$ is chosen, the ratio of the average $\bar{\sigma}$ of the scatter $\sigma_{i}$ within each base bin to the overall scatter $\sigma$, i.e.,

$$
\Theta=\frac{\bar{\sigma}(T)}{\sigma}=\frac{1}{N_{i}} \sum_{i=1}^{N_{i}} \frac{\sigma_{i}}{\sigma}
$$

assumes a minimum. For a period $T$ far off $T_{\text {rot }}$, the above scatter ratio has a value close to unity. The width and depth of the "absorption" peak that defines the minimum of $\bar{\sigma}(T) / \sigma$ near $T_{\text {rot }}$ depends among other quantities on the shape and the life time of the periodic signals in the time series $e(t)$. Further, the length of the time base $t_{n}-t_{0}$ of $e(t)$ and the existence of periodic signals with period $T^{\prime}$ close to $T_{r o t}$ also contribute to modify the width of this peak. 
The basic idea of the PDM method is to associate variations of the emissivity when they reappear in the same latitude and radius bin after one solar rotation. Consequently, the method is sensible only to variations with a correlation time exceeding $T_{r o t}$ (method $\mathrm{B}_{0}$ mentioned above). On the other hand, when mapping the east onto the west limb by including the $180^{\circ}$ phase correction (method $\mathrm{B}_{1}$ ), a lifetime for transient structures of only $>T_{\text {rot }} / 2$ is necessary to influence the period estimate that the method yields. In terms of Wang and Sheeley's model (Wang \& Sheeley, 1994), the effective time lag of the observations is $\sim 27$ days for method $\mathrm{B}_{0}$ and $\sim 14$ days for method $\mathrm{B}_{1}$.

\subsubsection{Results}

The top panel of Figure 5.2 shows two typical time series obtained according to Section 5.2.1 (method $\mathrm{B}_{0}$ ). The corresponding periodograms obtained with the PDM method are shown in the bottom panel. The left panel results from the analysis of the time series corresponding to $r=1.26 \mathrm{R}_{\odot}$ and position angle $P=90^{\circ}$, whereas the one in the right panel from the time series at $r=1.26 \mathrm{R}_{\odot}$ and $P=45^{\circ}\left(\operatorname{method} \mathrm{B}_{0}\right)$. One main "absorption" peak at around 27 days is clearly identifiable.

In order to build the rotation map of the green emission corona between $1.1 \mathrm{R}_{\odot}$ and $2.2 \mathrm{R}_{\odot}$, we have to determine the minimum of the main "absorption" peak for all of the periodograms obtained. This was done by fitting to each main peak in the periodograms a Gaussian curve plus a second-degree polynomial to represent the background. The position of the minimum of the resulting fit is chosen as the synodic rotation period. The rotation map derived with method $\mathrm{B}_{0}$, i,e, the east and west limbs treated independently, is shown in Figure 5.3. The FWHM of the Gaussian portion of the fitting curves is represented as function of position angle and radial distance in Figure 5.4.

\section{The uncertainty of the period estimation}

The uncertainty of the period estimation can be guessed from the difference between east and west limb results of method $\mathrm{B}_{0}$ (Figure 5.5). This method treats both limbs independently. Therefore, the method $\mathrm{B}_{0}$ may produce asymmetries with respect of the two limbs, as can be seen in Figure 5.3. Besides the noise in the observations, the discrepancy is probably due to short lived variations in the Fe XIV emission with life times less than half a rotation period.

\section{Coronal and photospheric rotation in brief}

In order to put our results in context with those obtained by other methods, we plot in Figure 5.6 the synodic rotation period (sidereal angular velocity) determined with method $\mathrm{B}_{0}$ at $1.15 \mathrm{R}_{\odot}$ averaged over both hemispheres as function of the solar latitude (red dashed-dotted line). The curves representative of each hemisphere, i.e., the red 

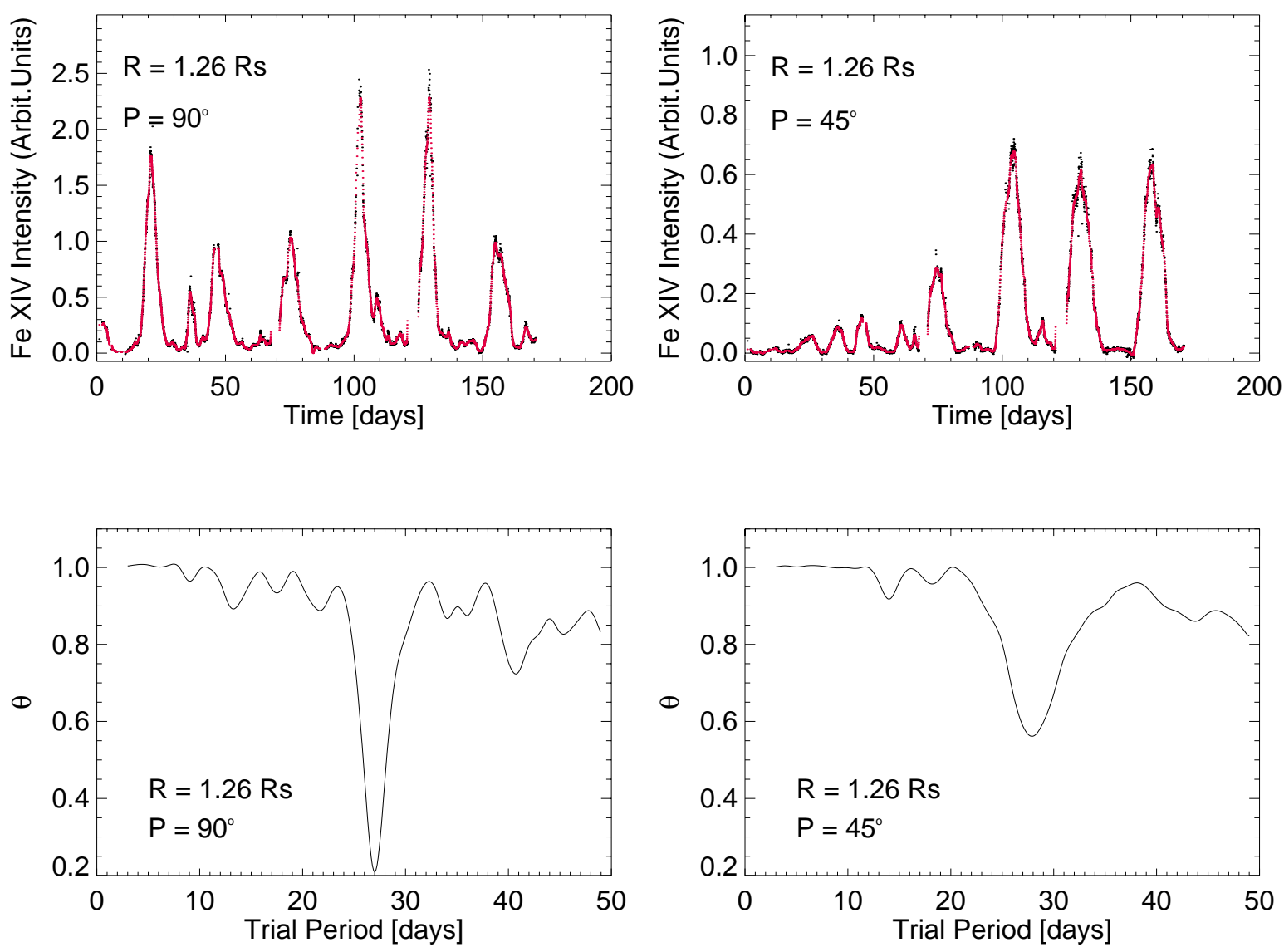

Figure 5.2: Top: Typical time series. They correspond to $P=90^{\circ}$ (i.e., equatorial east limb) and $P=45^{\circ}$ at $1.26 R_{\odot}\left(\operatorname{method} B_{0}\right)$, $P$ denoting position angle. The solid red line corresponds to the smoothed time series with the "à trous" algorithm (Starck et al., 1997). For more examples see also Figure 5.10. Bottom: Typical periodograms. The periodograms shown are the result of applying the PDM method to the respective smoothed time series shown in the upper panel. For details see text.

dashed line for the south hemisphere and the red dashed-dotted-dotted-dotted line for the north hemisphere, are the result of averaging the rotation periods corresponding to the same latitude on the east and west limb. In the same graph the following can be seen:

- Sidereal angular velocity curve derived from Snodgrass (1983). In his study, he determined the rotation rate of magnetic features by cross-correlating Mount Wilson magnetograms from observations made over successive days over the period 1967-1982 (dotted black line).

- Sidereal angular velocity curve derived from Howard et al. (1983). He determined the plasma rotation rate from the analysis of spectra of the photosphere (dashed black line).

- Sidereal angular velocity curve determined by Stenflo (1989). That curve was obtained from an autocorrela- 


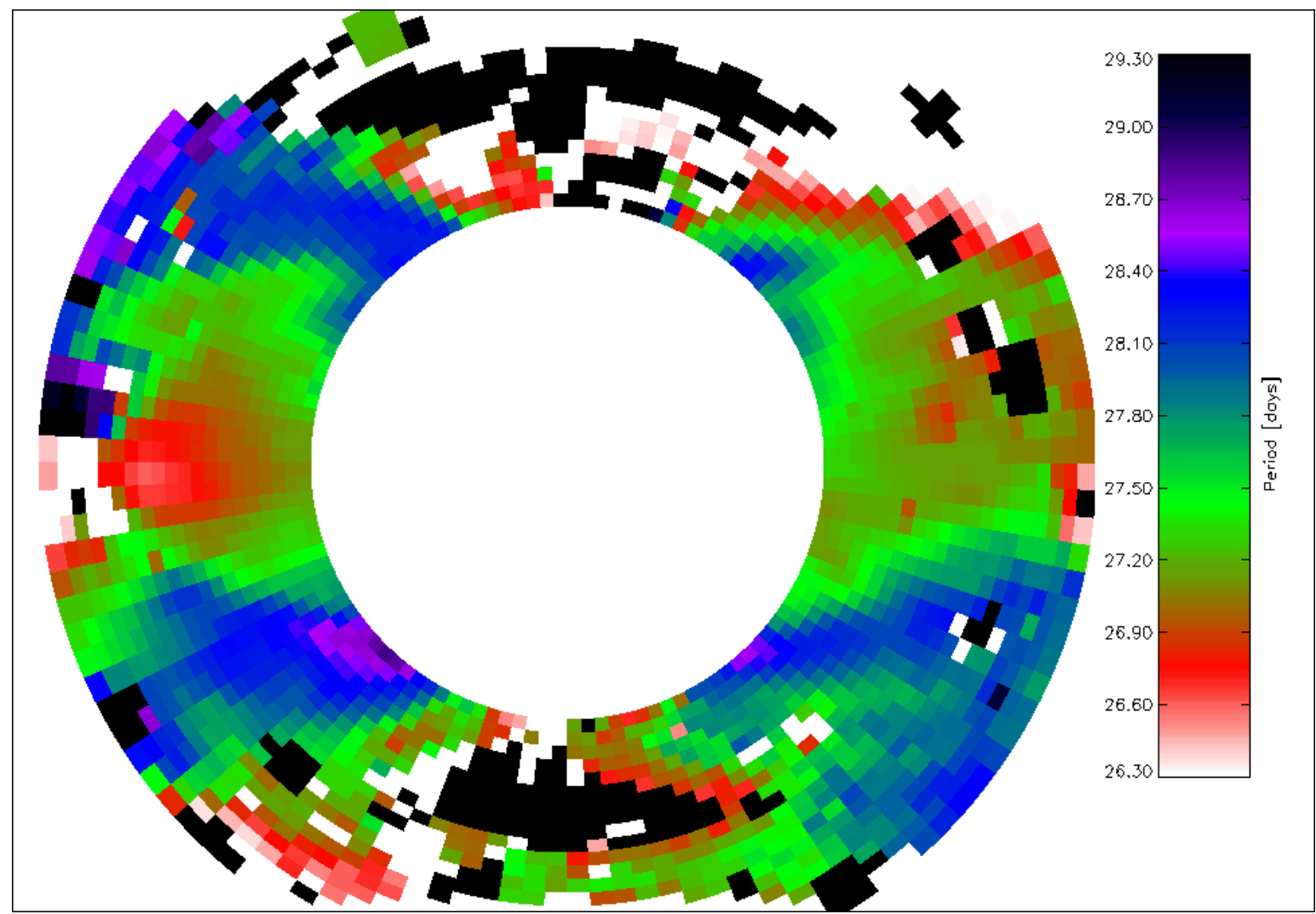

Figure 5.3: Color-scale representation of the variation of the computed periods (Method $B_{0}$ ) with position angle and radial distance.

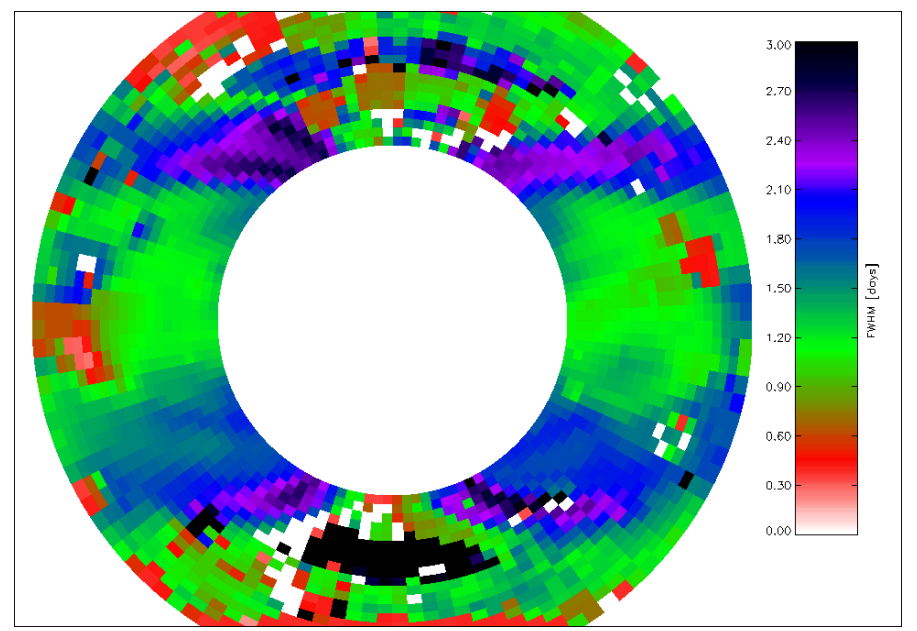

Figure 5.4: Color-scale representation of the FWHM of the peak corresponding to the main periodicity in the periodograms computed with Method $B_{0}$, as function of position angle and radial distance.

tion analysis performed on a 26-year synoptic data set (1959-1985) of magnetic fields sampled at the Sun's central meridian (dashed-dotted black line). Note the inversion of the angular velocity curve above about $\sim 50^{\circ}$ latitude.

- The averaged synodic rotation periods (denoted by the symbols + and $*$ in the graph) determined by Sime 


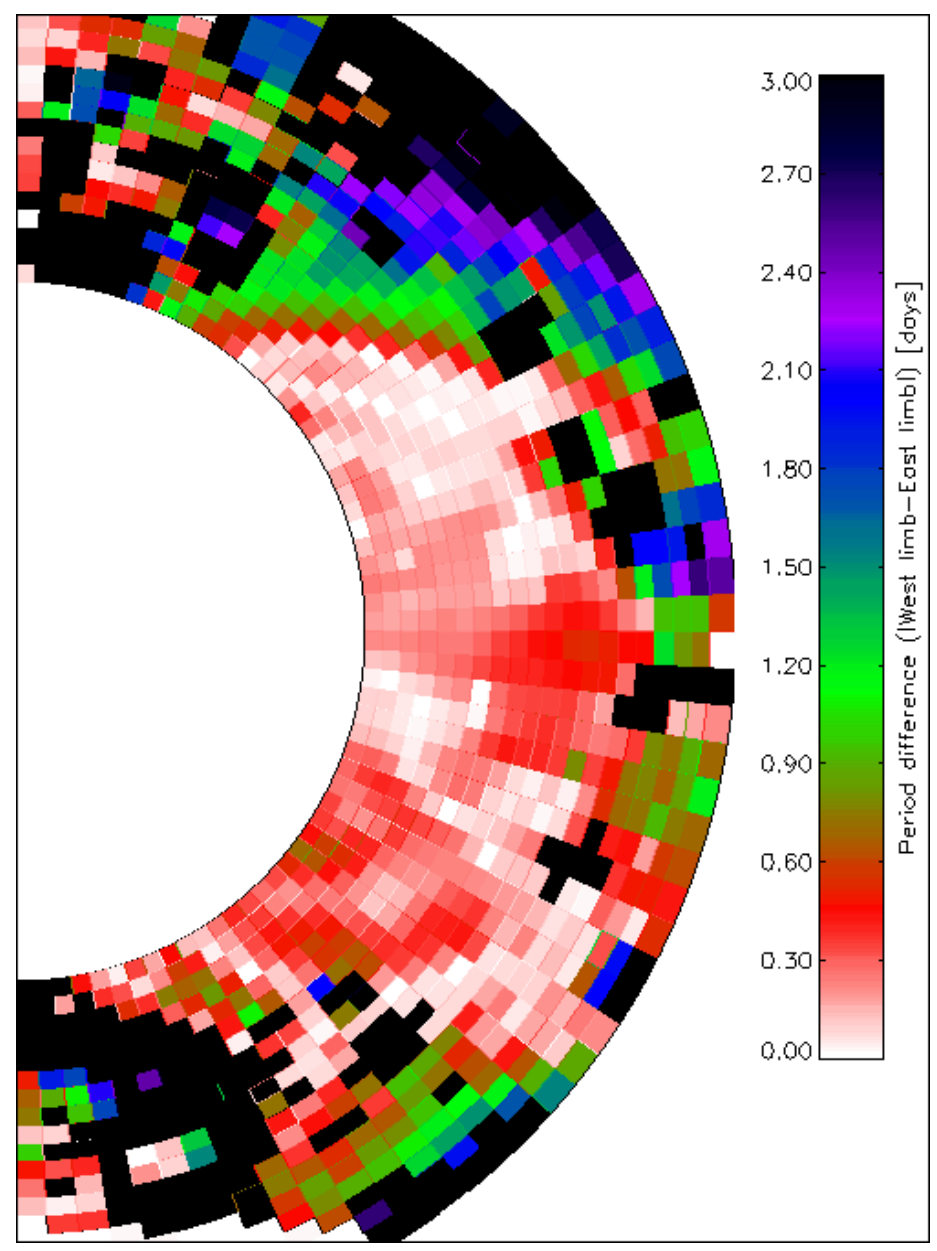

Figure 5.5: Color-scale representation of the uncertainty of the period estimates as obtained with method $B_{0}$. The uncertainty is estimated by taking the absolute value of the difference between the western and eastern limbs.

et al. (1989) from the analysis of green line coronal data obtained with the Sacramento Peak $40 \mathrm{~cm}$ coronagraph (see Section 3.5) at a radius of $1.15 \mathrm{R}_{\odot}$ between 1973 and 1985 . For the analysis, they divided the data into yearly intervals and then averaged into bands of latitude $\left(15^{\circ}\right.$ in extent) centered at $75^{\circ}, 60^{\circ}, 45^{\circ}$, $30^{\circ}$, and $15^{\circ}$ latitude in each hemisphere, and the solar equator. The green dashed-dotted line represents the sidereal angular velocity curve averaged over both hemispheres.

The common feature between Method $\mathrm{B}_{0}$, Stenflo (1989), and Sime et al. (1989) is that the three respective curves clearly show a polar spin-up at certain latitudes. The three methods mentioned above are based in the analysis of the recurrence of the either magnetic or intensity pattern after several solar rotations, i.e., only those structures with lifetime $>T_{\text {rot }}$ influence the period estimate. On the other hand, Snodgrass (1983) used crosscorrelation of magnetic patterns recorded with a time separation of 1-4 days to determine the rotational displacement of the features. Thus, the time scales involved in the two kind of determinations differ by an order of magnitude. For a further example see Section 5.3. 


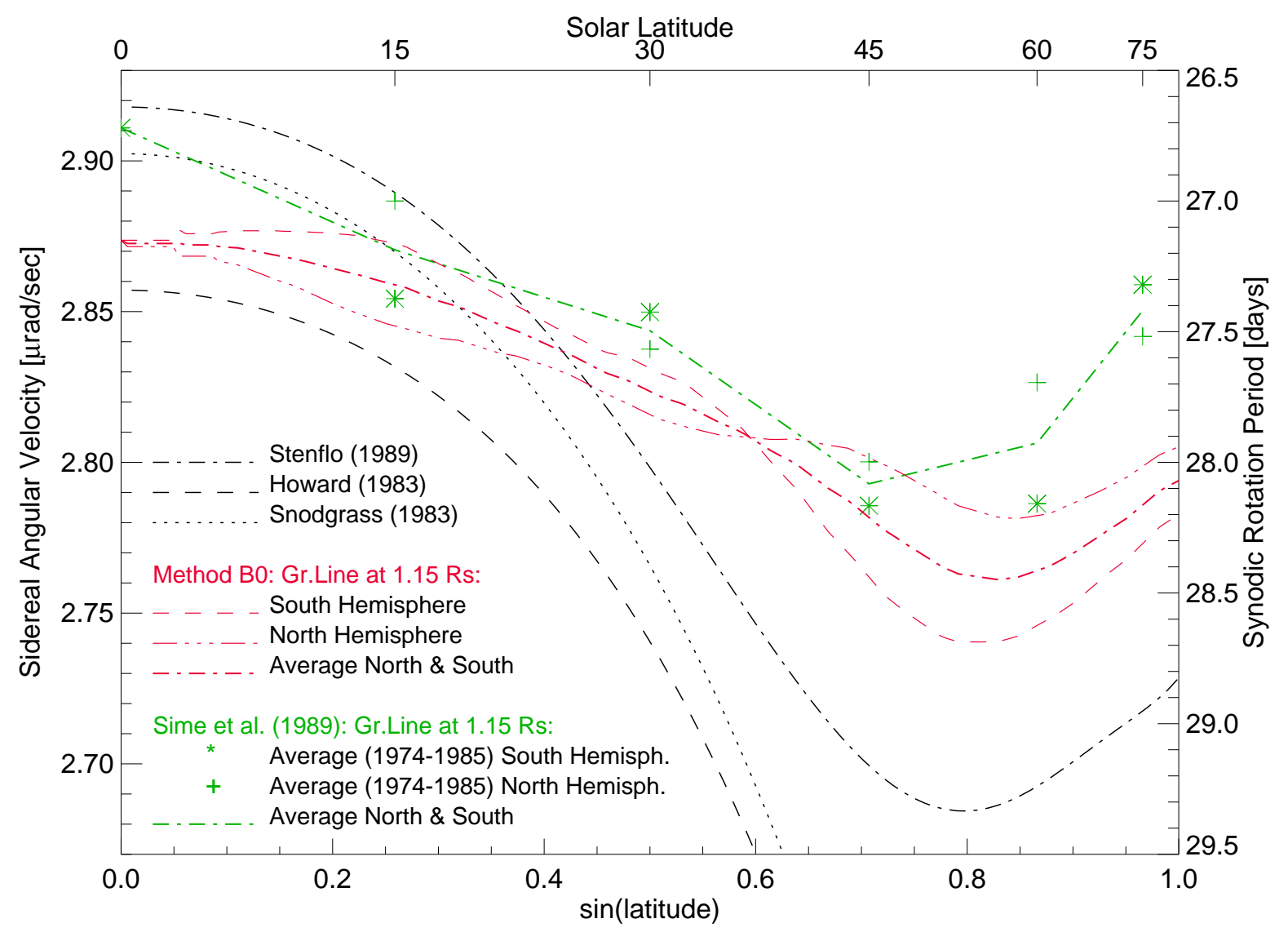

Figure 5.6: Sidereal angular velocity of rotation (synodic rotation period) vs sine of the latitude (solar latitude) at i) photospheric levels as derived from the analysis of a) the recurrence of the magnetic field pattern (Stenflo, 1989), b) Doppler shifts of photospheric Fraunhofer lines (Howard, 1983), and c) the longitude displacement of the photospheric magnetic field (Snodgrass, 1983); and ii) at $1.15 R_{\odot}$ as obtained from the analysis of the recurrence of the green line emission pattern: a) Sime et al. (1989) in green, and b) Method $B_{0}$ (this work) in red. For details see text.

\subsubsection{Discussion}

The increasing FWHM with latitude of the peak at $\sim 27$ days in the periodograms obtained with method B 0 (Figure 5.4) led us to analyze in deeper detail our results.

\section{Effect of green line transients}

In order to remove transient events such as green line transients (see, e.g., Section 4.4) which could alter the stationary behaviour of the time series, we reconstructed each of the time series by taking only one image per day. This image was obtained as the median of all of the images on the corresponding day. Then, the PDM method 
was applied to these time series (method $\mathrm{B}_{01}$ ). For comparison, Figure 5.7 shows the position angle and radial dependence of the difference of the computed periods as obtained with methods $\mathrm{B}_{0}$ and $\mathrm{B}_{01}$.

Since the absolute value of the difference between the period estimates with methods $\mathrm{B}_{0}$ and $\mathrm{B}_{01}$ is of the order of the estimated uncertainty (compare figures 5.5 and 5.7), we can neglect the effect that green line transients could have on the period estimation and consider both methods as equivalents.

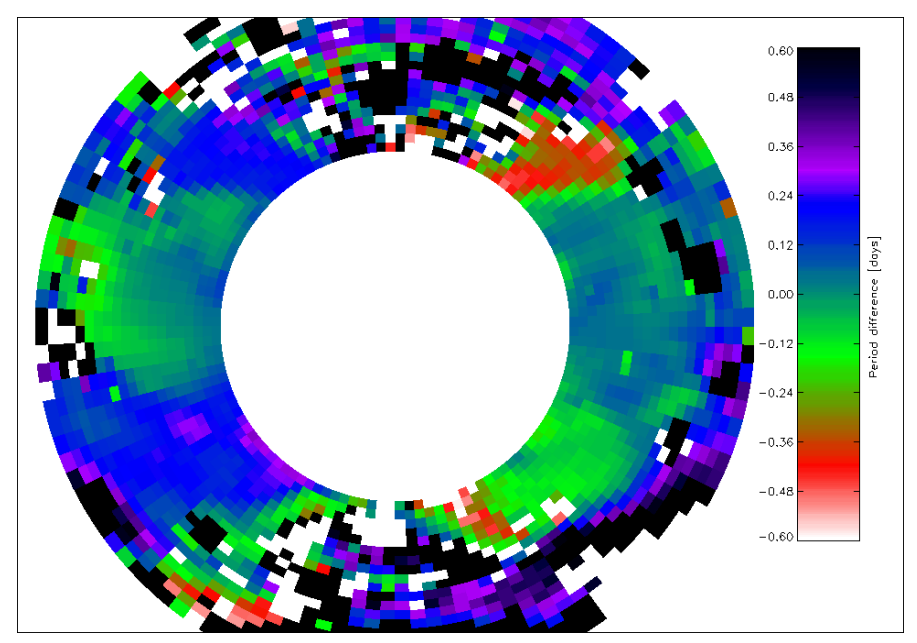

Figure 5.7: Color-scale representation of the difference obtained when computed the periods with the methods $B_{0}$ and $B_{01}$, showing its variation with position angle and radial distance.

\section{Effect of emissivity variations with correlation times between 0.5 and $1 \mathbf{T}_{\text {rot }}$}

Figure 5.8 shows a comparison between the periodograms obtained from time series built according to method $B_{0}$ (black and red solid lines) and method $B_{1}$ (dashed-dotted green lines) for several latitudes and solar distances. As exemplified in that Figure, the combined limb period estimates obtained with method $\mathrm{B}_{1}$ always lies between the two individual limb values (method $\mathrm{B}_{0}$ ). This may be due to emissivity variations with correlation times between 0.5 and $1 \mathrm{~T}_{\text {rot }}$.

In most of the periodograms obtained with method $\mathrm{B}_{0}$, two small peaks at around 13.6 days and 41 days can be observed for both limbs (see, e.g., the periodograms plotted in red and black in Figure 5.8). On the other hand, it can also be seen that these two small peaks disappear in the periodograms obtained with method B ${ }_{1}$ (dashed-dotted green line). Method $\mathrm{B}_{1}$ is based on the assumption that the coronal structures reappear after half a solar rotation on the opposite limb. However, if a green line pattern exhibiting another periodicity exists, it will not have $180^{\circ}$ phase shift (i.e., be on the opposite limb) after $T_{r o t} / 2$ but will have a phase shift of $360^{\circ} \cdot 1 / T \cdot T_{r o t} / 2(T$ being the period of this pattern). In the case of the peak at $\sim 13.6$ days the phase shift after $T_{\text {rot }} / 2$ is $360^{\circ}$. Therefore, this pattern does not contribute to a periodicity of $\sim 13.6$ days since it is $180^{\circ}$ out of phase after half a solar rotation. Further, the peak at $\sim 41$ days is a harmonic of the peak at $\sim 13.6$ days. Hence, the absence of the peak at $\sim 13.6$ days explains the absence of its harmonic at $\sim 41$ days.

A closer inspection of Figure 5.8 also shows that the harmonic of $T_{r o t}$ at $\sim 54$ days practically disappears in the combined limbs method (dashed-dotted green line). Moreover, all of the harmonics of $T_{\text {rot }}$ at $2 n T_{\text {rot }}$ (with $n=$ $1,2, \ldots ; T_{\text {rot }}=\sim 27$ days) are practically not present in the periodograms obtained with method B ${ }_{1}$. Understanding 

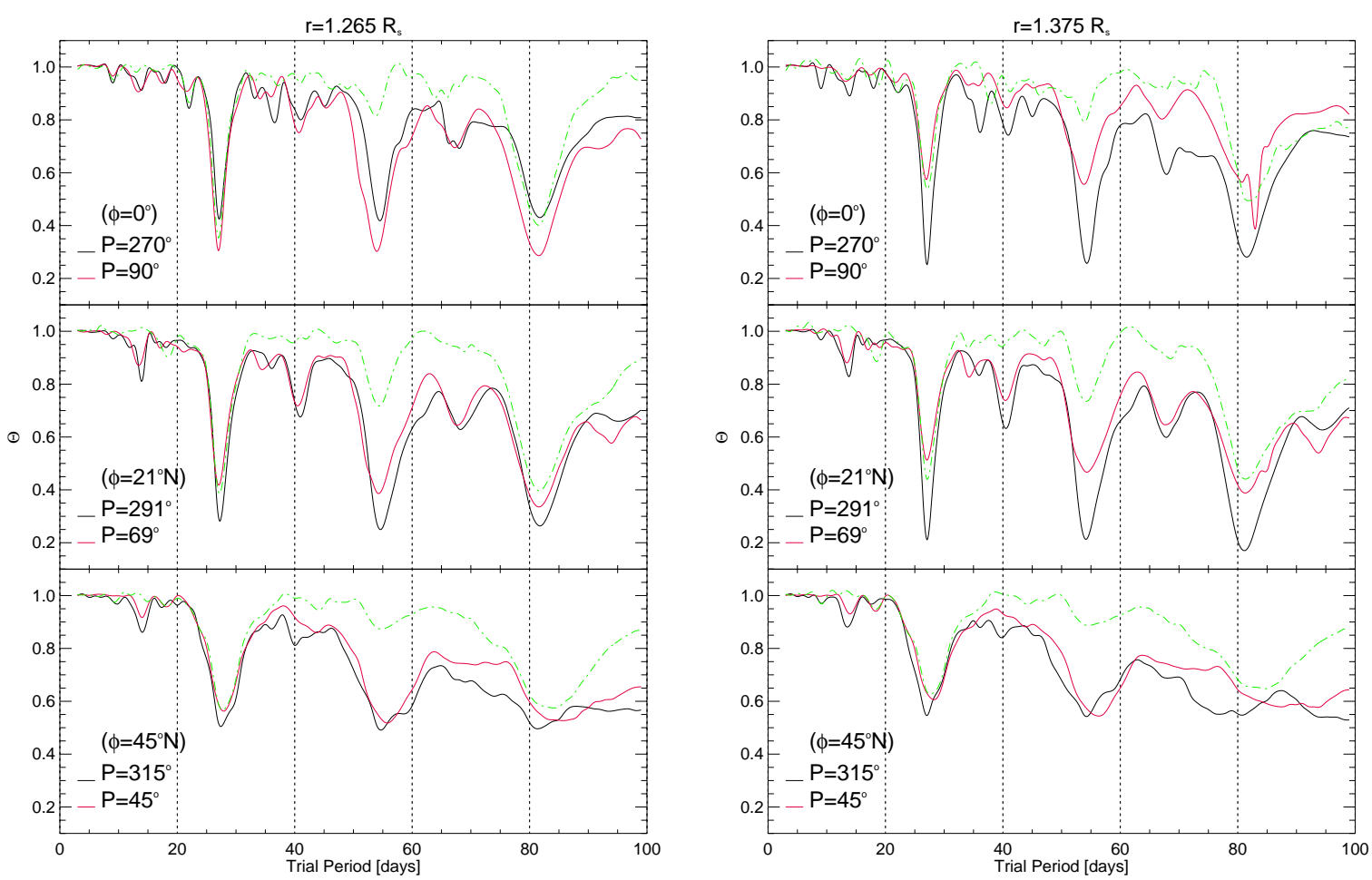

Figure 5.8: Comparison of methods $B_{0}$ and $B_{1}$ for several position angles, $P$, and solar distances, $r$. The solid black and red lines correspond to the periodograms obtained with method $B_{0}$ at the respective position angles. The dashed-dotted green line represents the periodogram obtained combining both limbs at the respective latitude (method $B_{1}$ ).

of how the PDM method is applied in that case and crosscorrelation of time series corresponding to opposite locations in the respective limbs help to understand the reasons of such a behaviour. The application of the PDM method to the combined limb data requires that for each trial period $T$, the respective time series in the opposite limb be shifted by $T / 2$ and then the statistic $\Theta$ (Equation 5.1) computed for the combined set. Therefore, $\Theta$ will have its minimum values at those periods where the time series corresponding to homologous positions in both limbs are in phase after shifting one of them by $T / 2$. The periods at which that occurs, can be calculated by taking into account the crosscorrelation of the time series corresponding to opposite locations. In other words, the time lags at which the maximum of the crosscorrelation, i.e., $(2 n+1) T_{\text {rot }} / 2$, matches the $T / 2$ shift of the time series corresponding to the opposite limb, will be the periods at which the statistic $\Theta$ has its minimum values. For the sake of clarity, an example assuming $T_{\text {rot }}=27$ days is shown in Table 5.1. It can be seen that only at the position of the fundamental period and the harmonics corresponding to $n=3,5, \ldots,(2 n+1) T_{r o t}, \ldots$ the two time series are in phase.

\section{On the existence of other periodicities}

In Figure 5.9 we plotted several periodograms as function of solar latitude. They were obtained after applying the PDM method to time series built according to method $B_{0}$. Figure 5.9 clearly shows that the peak at around 


\begin{tabular}{lccccc}
\hline \hline$n$ & $\mathbf{1}$ & 2 & $\mathbf{3}$ & 4 & $\mathbf{5}$ \\
$n T_{\text {rot }}$ (days) & $\mathbf{2 7}$ & 54 & $\mathbf{8 1}$ & 108 & $\mathbf{1 3 5}$ \\
$n / 2 T_{\text {rot }}$ (days) & 13.5 & 27 & 40.5 & 54 & 67.5 \\
Max. Crosscorr (days) & 13.5 & 40.5 & 67.5 & 94.5 & 121.5 \\
\hline \hline
\end{tabular}

Table 5.1: Example to show why the harmonics at $2 n T_{\text {rot }}$ ( $T_{\text {rot }}$ assumed to be 27 days) disappear in the periodograms corresponding to method $B_{1}$ (dashed-dotted green line in Figure 5.8). The numbers in bold face in the second row show the periods at which a minimum value of the statistic $\Theta$ is expected. The numbers in italics in the third row denote the shift to be applied to the time series corresponding to the opposite limb before applying the PDM method to the combined limb time series. The values of $n$ at which the values in the third row match the values in the fourth row are the harmonics which are expected in the combined limb method $\left(B_{1}\right)$.

27 days, here labelled $\mathrm{A}$, is broader and no longer symmetric as we increase in latitude. Note also the other peaks C, D, E, F, and G, and the pseudo-peak B. In order to understand the physical reasons that could lead to widening and increasing asymmetry of the peak $\mathrm{A}$ as well as to know the nature of the other peaks indicating potential periodicities, we made several critical tests:

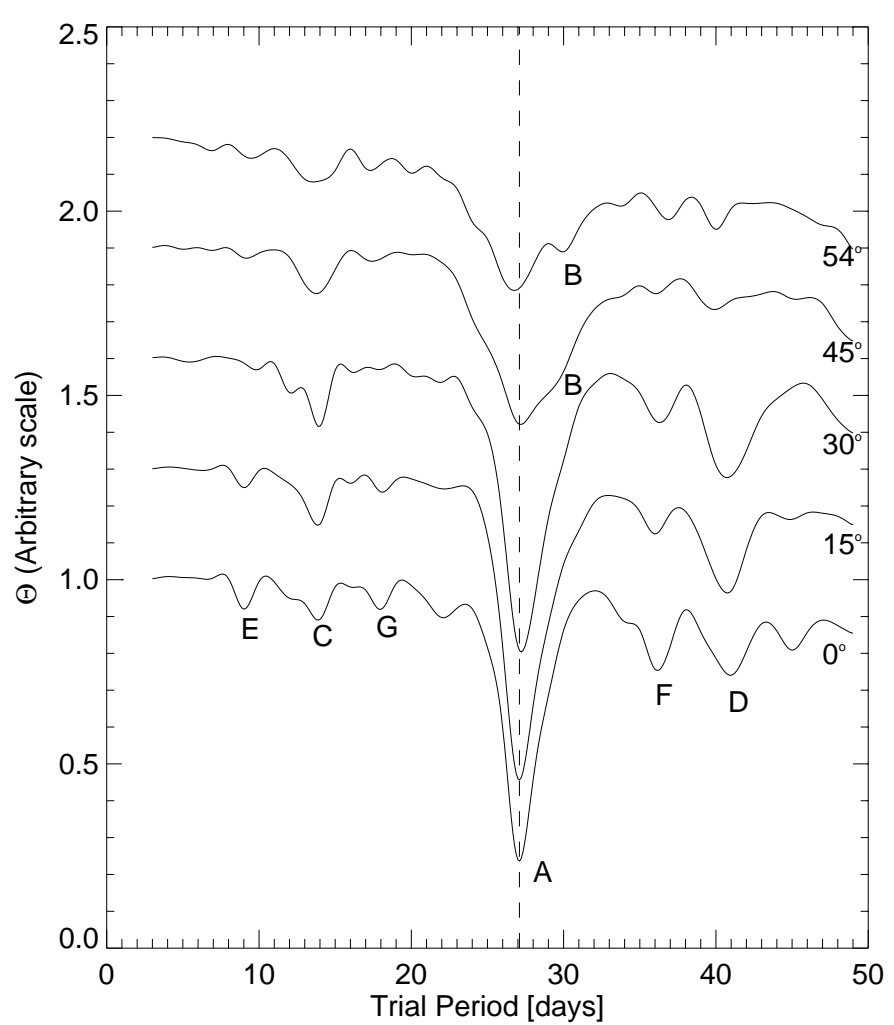

Figure 5.9: Periodograms as obtained with Method $B_{0}$ for $0^{\circ}, 15^{\circ}, 30^{\circ}, 45^{\circ}$, and $54^{\circ}$ North latitude (West limb) at $r=1.32 R_{\odot}$. Note the appearance of the peak labelled $B$ at higher latitudes. For details see text. 


\section{Effect of the spectral window ${ }^{3}$ :}

The observing times define a window. This window, convolved with the signal, determines the shape of the periodograms. If one observes a non-variable object each day at exactly the same time e.g., the periodogram analysis on it will return a significant peak at 1 day (and corresponding harmonics). This means, there is a periodic signature from the sampling frequency itself. In that respect, unevenly sampled time series have an advantage. Nevertheless, in order to identify the potential presence of interference peaks due to the convolution of the discrete Fourier transform of the data with the so-called spectral window, we scrambled the data points of the time series $e(t)$, by randomly reassigning them to the actual observation times. This reassignment preserves the window function and the variance of the data but destroys any periodic signal which might be present. In 30 trials, calculation of the periodograms never produced the periodicities seen in the actual data. Therefore, this test discards the effects the sampling time window could produce.

\section{Statistical significance:}

The statistical evaluation of detected periods resembles the evaluation of a theoretical curve fitted to experimental data (Lomb, 1976). The periodogram statistic $\Theta$ (Equation 5.1) measures the fit for a given period (frequency). The probability distribution of the statistic is then used to calculate the probability of obtaining the value of the periodogram higher (lower) than the actual observed value from a hypothetical pure noise signal. Its complement probability is called significance level. In the PDM method, Schwarzenberg-Czerny (1989) and later Davies (1990) showed that the Fisher-Snedecor F-distribution proposed by Stellingwerf (1978) for the PDM statistic is applied incorrectly. The correct probability distribution appears to be a $\beta$ distribution (Schwarzenberg-Czerny, 1997).

If a large number of observations is available (our case), statistical significance is not a consideration. Indeed, the opposite problem is almost certain to arise: many possible periods resulting from aliasing and sidelobes, all of which are well above the noise level.

\section{Multiple significant peaks:}

In a periodogram several peaks can have significant depth according to the significance level. In our periodograms all the peaks mentioned above are significant to $99.9 \%$. Multiple significant peaks can be caused either by the presence of more than one periodic signal in the data or from modulation at one period. Either function could mimic the data and therefore produce two or more periodicities in the analysis. Alternatively, a true signal with period $T_{0}$ can cause peaks in the periodogram at periods other than $T_{0}$ because of the finite length of the data window and irregularities in the data spacing. This problem is usually referred to as spectral leakage ${ }^{4}$ (see, e.g., Horne \& Baliunas, 1986). One useful method to check whether significant peaks are physically real is to filter out the frequency corresponding to the most significant peak and then recompute

\footnotetext{
${ }^{3}$ The so-called term "spectral windows" is used to refer to the Discrete Fourier Transform (DFT) of the sampling time.

${ }^{4}$ There are several forms of spectral leakage. Leakage to nearby frequencies (sidelobes) is due to the finite total interval over which the data is sampled. Leakage to distant frequencies is due to the finite size of the interval between samples. In particular, the well known phenomenon of aliasing is a leakage of power from high to much lower frequencies. In this case, anything from a slight to major unevenness in the spacing between samples substantially reduce aliasing (Scargle, 1982).
} 
the periodogram. Scargle (1982) showed that this method is equivalent to remove a least-squares sine curve of the filter frequency from the data. A good computational method for subtracting a sinusoid can be found in Ferraz-Mello (1981). After filtering, any remaining significant peaks should not be due to spectral leakage of the filtered peak produced by interference with the spectral window function. This method is also useful if multiple periods are suspected, especially if they are too close to each other and the method is not able to resolve them.

In order to find other periodic components which might either be close to each other or due to spectral leakage of a main frequency, we followed Ferraz-Mello (1981) by filtering the time series and eliminating the sine wave whose period has been selected. The procedure was executed at first in the north-eastern limb. The time series obtained according to method $\mathrm{B}_{0}$ were analyzed in the radial direction in increasing steps of $0.17 \mathrm{R}_{\odot}$ every $3^{\circ}$ in latitude. As it was said in Section 5.2.1, before applying the PDM method a smoothing technique based on the wavelet transform was applied to the time series. This technique employs the à trous algorithm (Starck et al., 1997) to reduce the noise level and get rid of wrong values. A sample of the time series used is shown in Figure 5.10. The corresponding periodograms superposed with the periodograms obtained after harmonic filtering at certain specific frequencies are shown in Figure 5.11. In that Figure, the solid black lines correspond to the periodograms obtained from the original time series. The results of the analysis can be outlined as follows:

a. Harmonic filtering of a component with 27.2 days periodicity:

The red solid lines in Figure 5.11 correspond to the periodograms obtained from the time series after filtering the 27.2 days periodic component.

* Suppression of the main peak at 27.2 days makes the peak at $\sim 13.6$ days more significant.

* After reaching a maximum significance at around $40^{\circ}$ latitude, this peak at $\sim 13.6$ days gets weaker and is finally no longer discernible around $50^{\circ}-54^{\circ}$, depending on solar distance.

b. On further harmonic filtering of a component with 13.6 days periodicity:

The corresponding periodograms are plotted in green in Figure 5.11.

* A new peak at $\sim 9$ days turns significant at equatorial latitudes, becoming negligible beyond $12^{\circ}-18^{\circ}$ latitude.

* At $\sim 24^{\circ}$ latitude another peak begins to be discernible at $\sim 30$ days in the periodograms corresponding to time series close to the limb.

* On the other hand, in the periodograms corresponding to time series at larger solar distances, the peak at $\sim 30$ days becomes visible at higher latitudes.

* As can be noted in Figure 5.11, at $\sim 36^{\circ}$ latitude the peak at $\sim 30$ days can be well appreciated at all of the solar distances analyzed.

* The appearance of this third peak at $\sim 30$ days remains until $50^{\circ}-60^{\circ}$ latitude, depending on solar distance. 


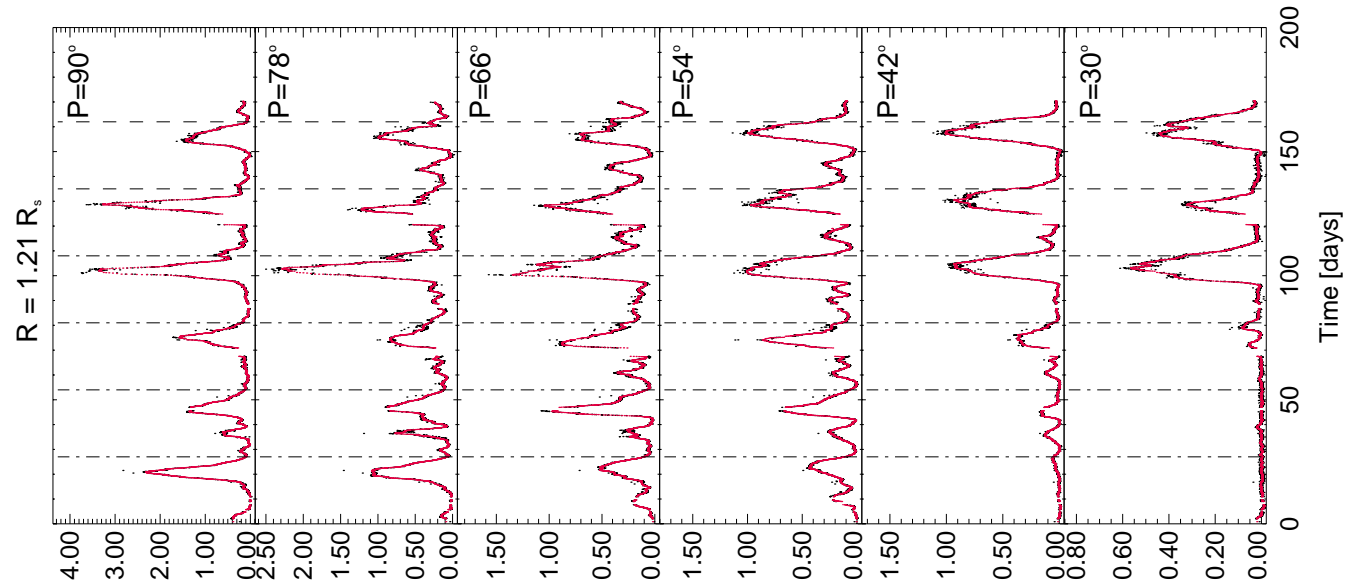

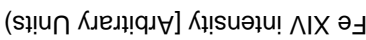

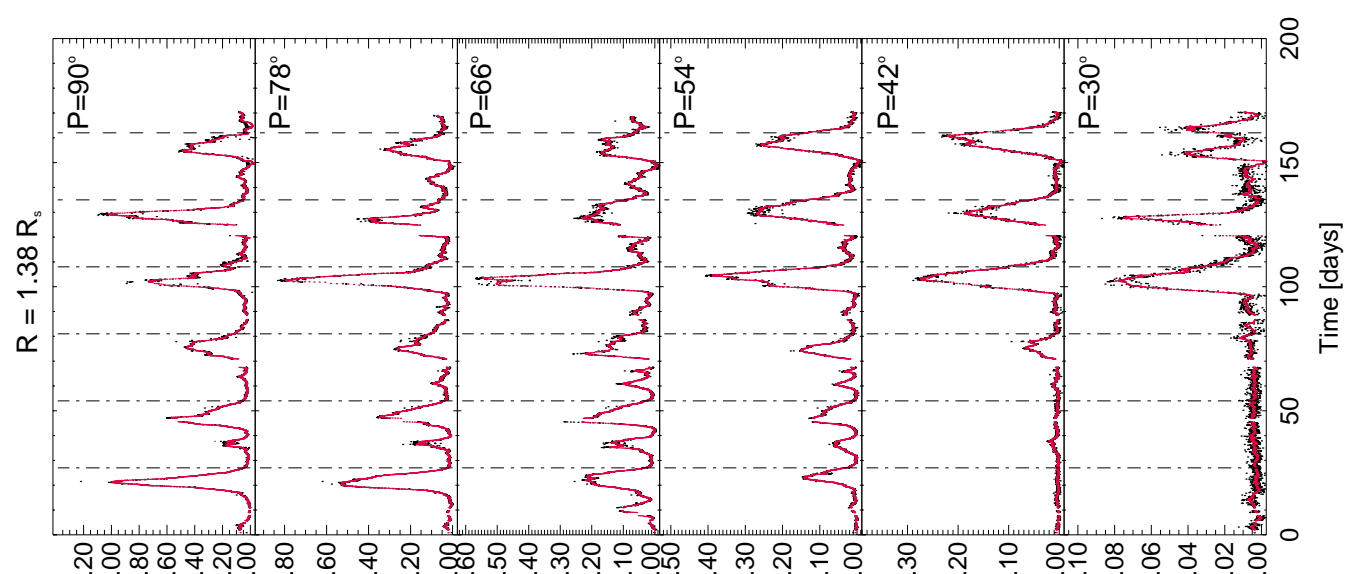

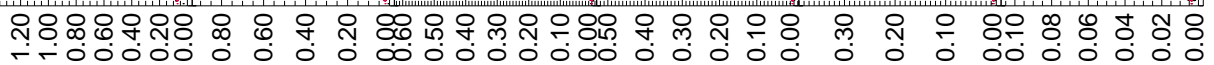

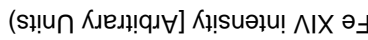

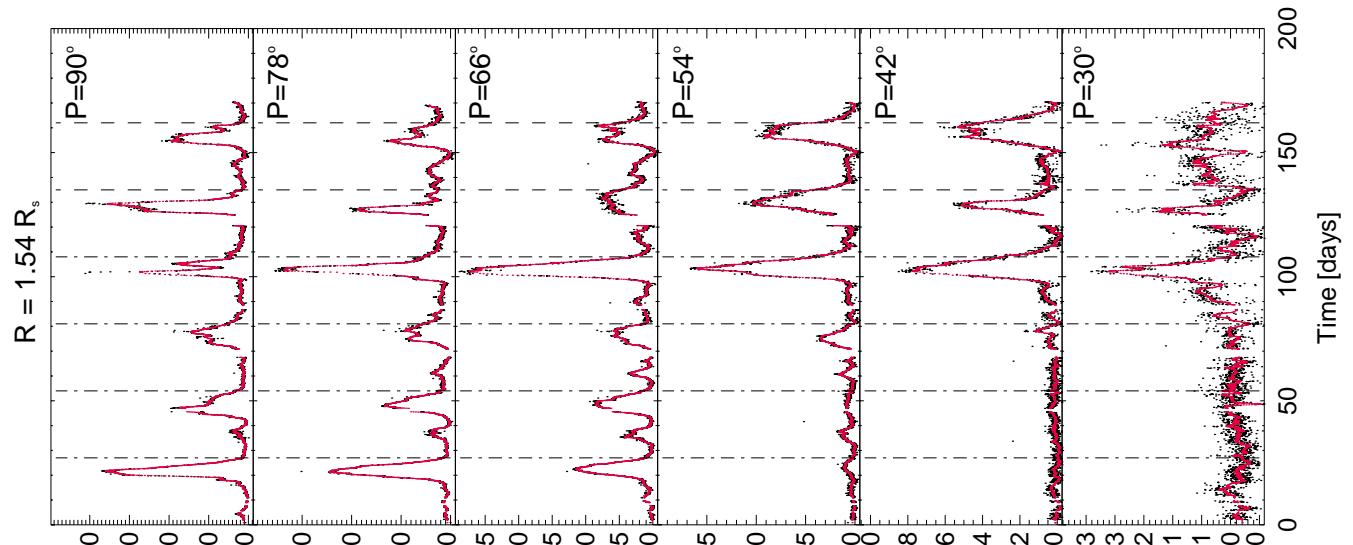

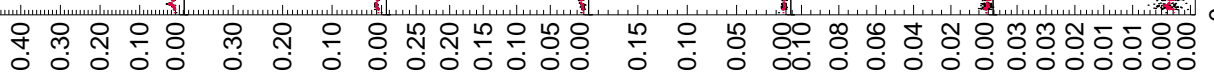

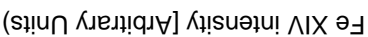

Figure 5.10: The line-of-sight integrated intensity for several position angles $P$ and solar distances $R$ as observed by LASCO/C1 during the period May-October, 1996 (black dots). The solid red line corresponds to the filtered time series with the "à trous" algorithm (Starck et al., 1997). This algorithm was applied to the time series before applying the PDM method in order to get rid of wrong values and reduce the noise level. 


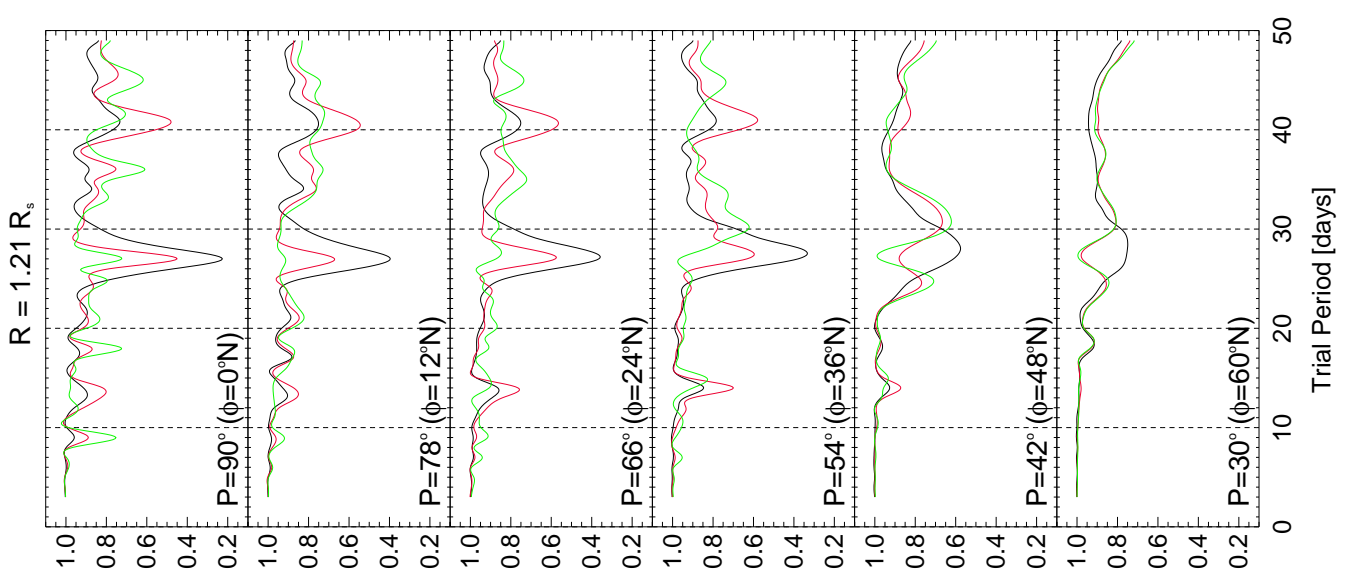

$\Theta$

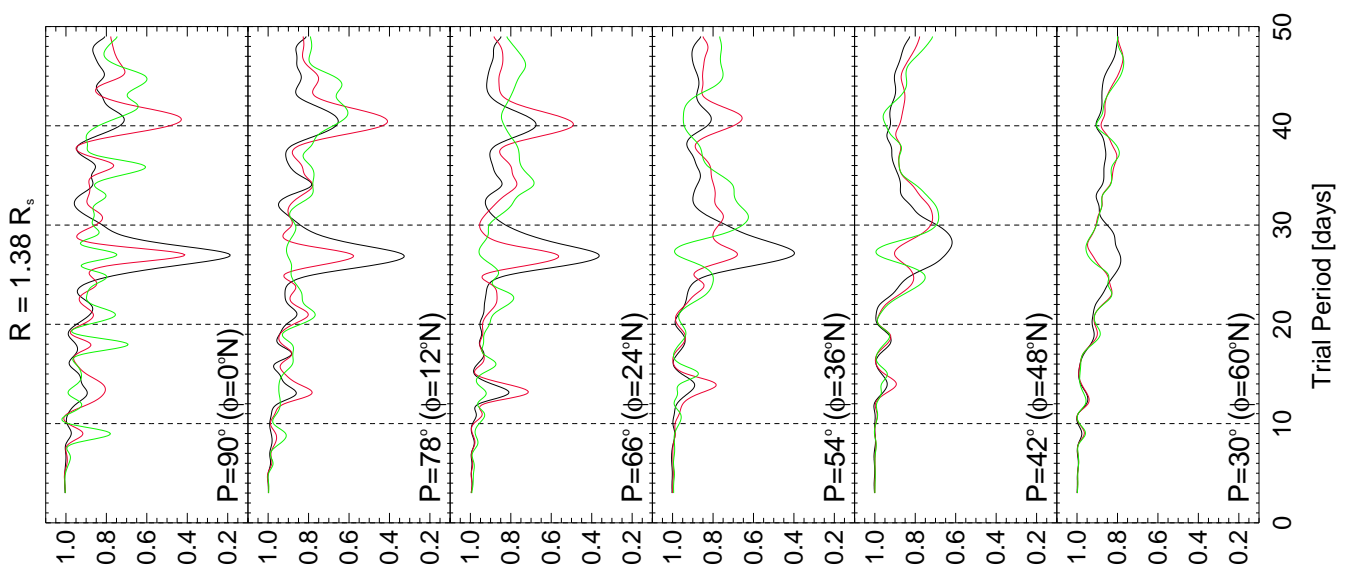

$\Theta$

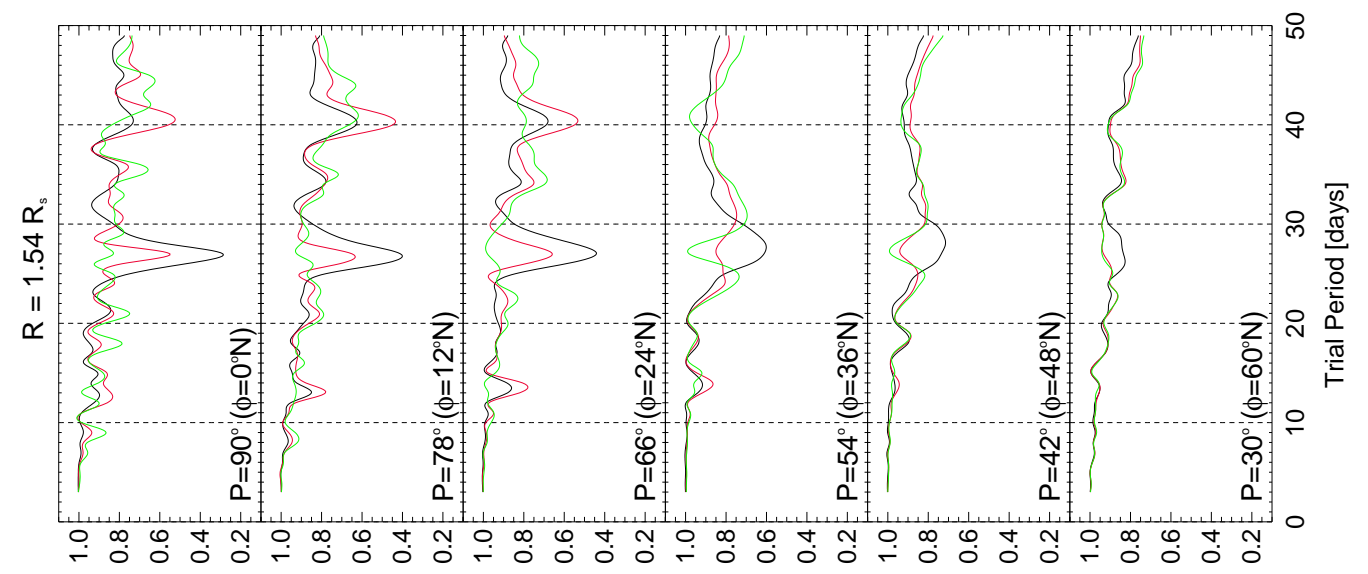

$\Theta$

Figure 5.11: Corresponding periodograms obtained after applying the PDM method to the smoothed time series i) as shown in Figure 5.10 (solid black line), ii) after removal of a 27 days periodic component (red solid line), and iii) after removal of an additional periodicity of 13.6 days (green solid line). For details see text. 
* At higher latitudes only a broad peak at around 27.2 days is found.

We repeated the procedure in the other three quadrants. The shape of the periodograms obtained showed a similar behaviour, i.e., existence of three peaks as in the previous case with some little differences with respect to the latitudes at which these peaks appear. Therefore, this analysis confirms that the origin of the most significant peaks in the periodograms is not due to spectral leakage.

\section{The PDM method applied to synthetic data:}

The previous analysis showed that the peaks A, C, and the pseudo-peak B, are not the result of spectral leakage (notation used in Figure 5.9 to denote the peaks at $\sim 13.6, \sim 27.2$, and $\sim 30$ days). However, it is not clear yet whether they are due to i) several periodic components in the time series or ii) modulation produced by the evolution (appearance, disappearance) of active areas. This can be analyzed by using surrogate time series $^{5}$ that mimic the typical shape of the periodograms obtained.

The representation of several periodicities in the data, can be most easily represented by the sum of several sine curves with known frequencies combined with noise whose variance equals that of the observational data. In this interpretation, two or more active areas, presumably at different latitudes, rotate at different rates and produce a modulation curve reminiscent of an interference pattern. On the other hand, the modulation produced by the growing of an active region e.g., can be represented by a monochromatic sine curve multiplied by an exponential function.

Several synthetic curves with the features aforementioned were built and tested. The periodograms resulting of applying the PDM method to a pair of these synthetic functions are shown in the upper panels of Figure 5.12. For the first case, (top left), the sum of three sinusoidal functions were selected with the following frequencies: $1 / 13.6,1 / 27.2$, and $1 / 30$ days $^{-1}$. In order to better understand the periodograms, we show in the graph three periodograms, each of them corresponding to the synthetic time series built with a different initial phase for the 30 days periodic component with respect of the other two. Note that the 30 days periodic component is not resolved. However, the shape of the main peak at the position of the unresolved periodicity at 30 days depends on the initial phase assigned to this component. In the second case, (top right), a sine curve with a frequency of $1 / 27.2$ days $^{-1}$ and an exponential function with a time constant of 70 days, to simulate the growing of an active region, were used. In both cases, the asymmetry of the peak at $\sim 27$ days can be noted. This asymmetry is due in the first case to the unresolved periodicity at 30 days, while in the second case is due to the modulation by the exponential function. The peak at $\sim 13.5$ days is, in the second case, also a by-product of the modulation. In the bottom panel of the same figure, the periodograms corresponding to the time series at $P=54^{\circ}$ and $r=1.21 \mathrm{R}_{\odot}$ are shown for comparison. Comparison of the top and bottom panels of Figure 5.12 does not allow us to choose any representation as the one explaining our results yet.

\footnotetext{
${ }^{5}$ Surrogate data are artificially generated data which mimic statistical properties of real data. In particular, isospectral surrogates have identical power spectrum as real data but with randomized phases.
} 
Therefore, we now filter out a specific frequency (according to Ferraz-Mello, 1981) from the surrogate time series in order to compare with the analysis performed in the previous item. The periodograms obtained after filtering out the 27- and 13.6-days components are shown in the upper panels of Figure 5.13 (left and right panels, respectively). As expected, in the first case, i.e., the sum of two sine curves, the peak denoting the $\sim 13.6$ days vanishes. In the second case, i.e., the modulation of a sine curve by an exponential function, the periodogram maintains the same shape, since the peak at $\sim 13.6$ days is an artifact of the modulation, not being a real component.

The effect of filtering out specific components resembles in the first case (i.e., coexistence of several periodic components) the behaviour observed in the previous item. Therefore, this analysis suggests the existence of several periodic components in the time series.

The observational conclusion drawn from the complete analysis is that

- the main peak labelled A in Figure 5.9 does correspond to a main periodic component in the time series, i.e., it depicts the rotation rate,

- the peak labelled $\mathrm{C}$ corresponds to an emission pattern exhibiting a $\sim 13.6$ days periodicity,
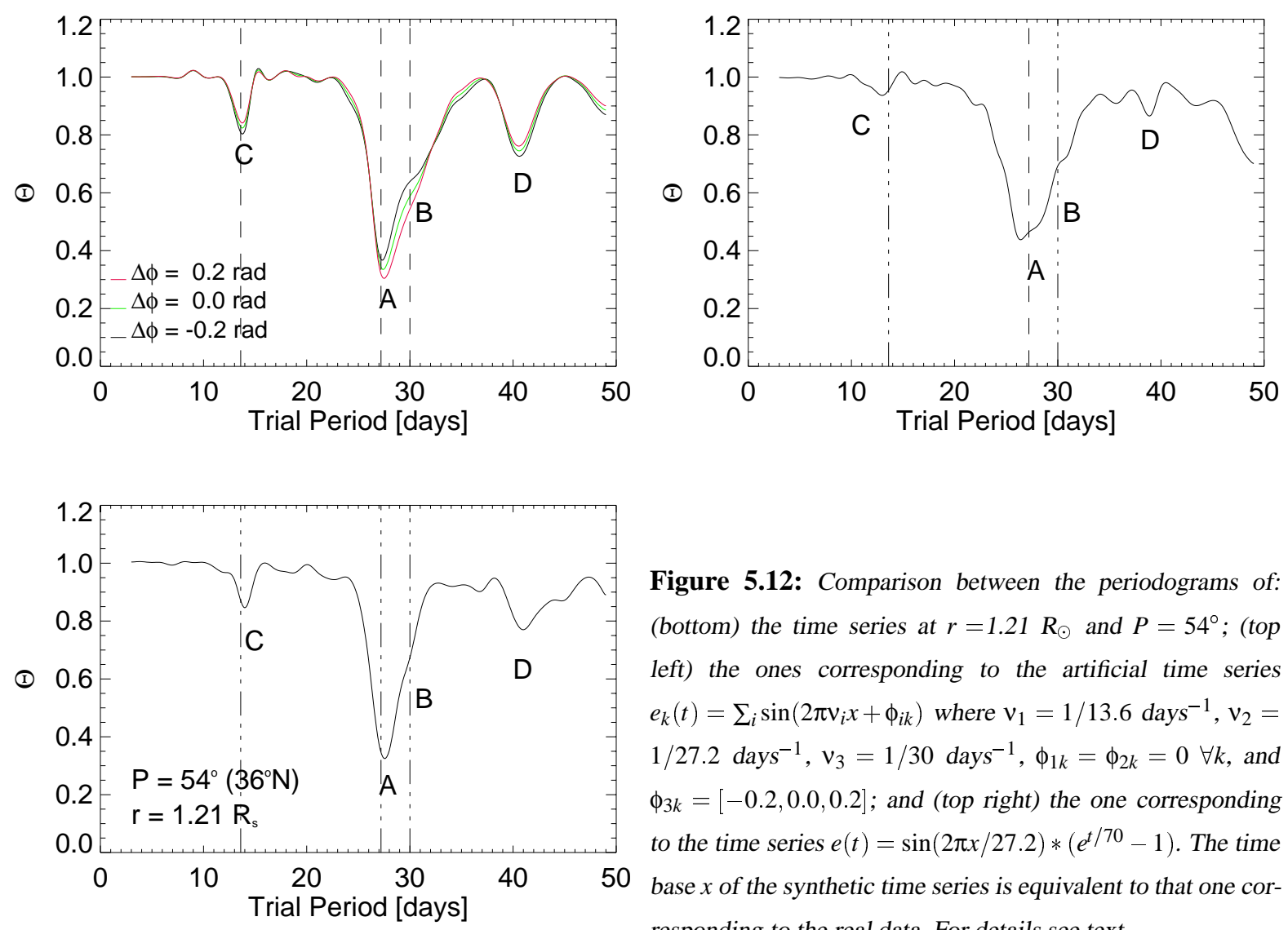

Figure 5.12: Comparison between the periodograms of: (bottom) the time series at $r=1.21 R_{\odot}$ and $P=54^{\circ}$; (top left) the ones corresponding to the artificial time series $e_{k}(t)=\sum_{i} \sin \left(2 \pi v_{i} x+\phi_{i k}\right)$ where $v_{1}=1 / 13.6$ days $^{-1}, v_{2}=$ $1 / 27.2$ days $^{-1}, v_{3}=1 / 30$ days $^{-1}, \phi_{1 k}=\phi_{2 k}=0 \forall k$, and $\phi_{3 k}=[-0.2,0.0,0.2]$; and (top right) the one corresponding to the time series $e(t)=\sin (2 \pi x / 27.2) *\left(e^{t / 70}-1\right)$. The time base $x$ of the synthetic time series is equivalent to that one corresponding to the real data. For details see text. 

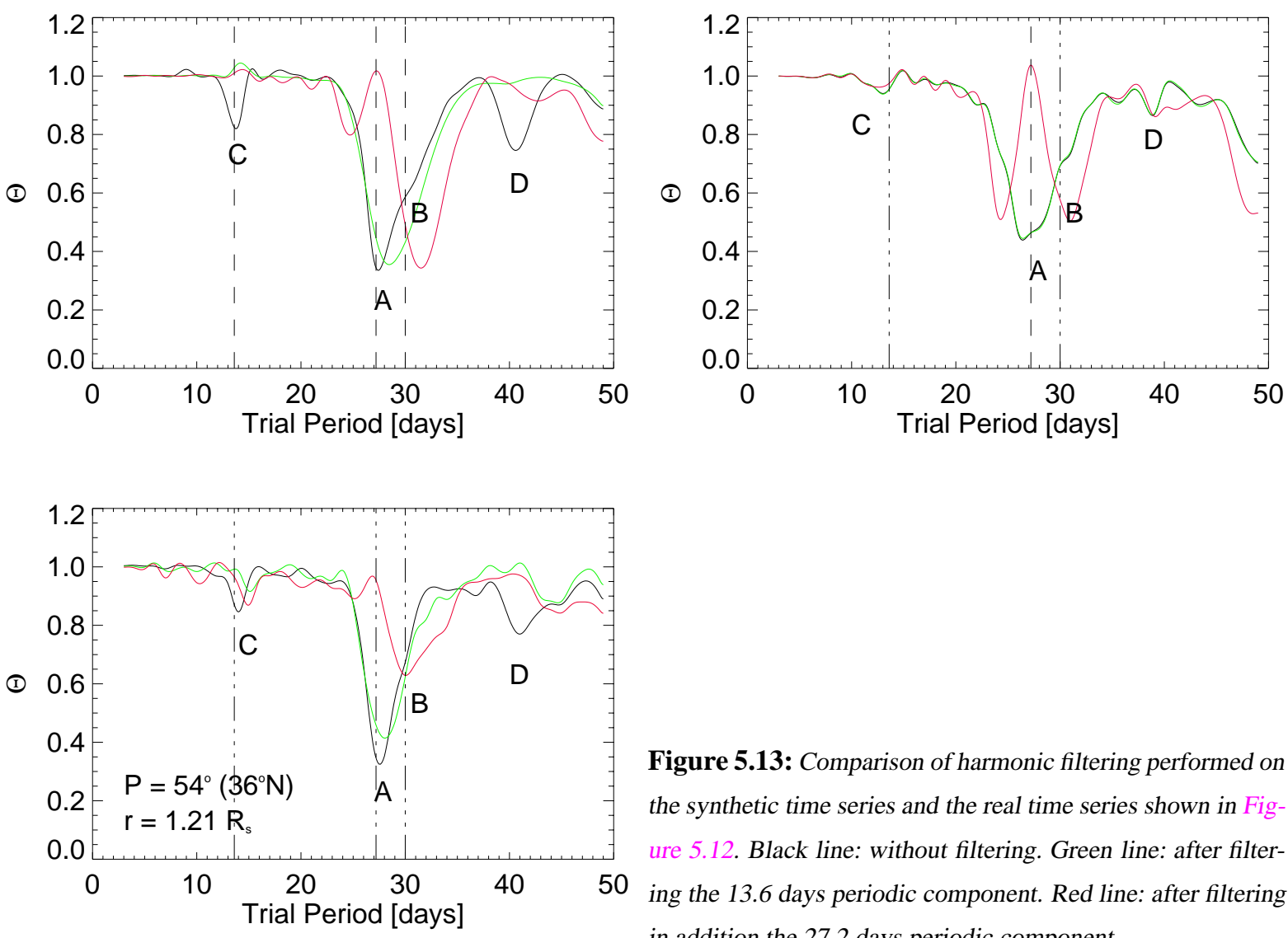

Figure 5.13: Comparison of harmonic filtering performed on the synthetic time series and the real time series shown in Figure 5.12. Black line: without filtering. Green line: after filtering the 13.6 days periodic component. Red line: after filtering in addition the 27.2 days periodic component.

- the peak labelled $\mathrm{D}$ is a harmonic of the peak $\mathrm{C}$, since removal of the corresponding frequency lets peak $\mathrm{D}$ disappear,

- the pseudo-peak B corresponds to the existence of another emission pattern, likely not resolved, rotating with a period close to 27 days, i.e., it would depict the rotation rate of real green line structures,

- finally, the peak $\mathrm{E}$ would correspond to an emission pattern exhibiting a $\sim 9$ days periodicity, $\mathrm{G}$ and $\mathrm{F}$ being harmonics of it.

\subsubsection{Summary}

The methods we used ( $\mathrm{A}$ and $\mathrm{B}$ in Paper $\mathrm{I}, \mathrm{B}_{0}, \mathrm{~B}_{01}$, and $\mathrm{B}_{1}$ ) reveal the recurrences of the green line pattern. The green line emission is observed to occur above either active regions or photospheric magnetic neutral lines. The persistence of mid-latitude streamers above magnetic neutral lines in the photosphere has already been shown by, e.g., Schwenn et al. (1997). It also confirms previous results of Guhathakurta et al. (1993) and Guhathakurta \& Fischer (1994) who found two zonal bands of high coronal temperature at latitudes around $50^{\circ}$. These bands tend to lie in regions above a magnetic neutral line and bright features in coronal polarization brightness. 
Basically, the main difference of the present work with that of Paper I lies in the way we determined the position of the peak corresponding to the main period. In Paper I, the position of the minimum of the main peak around 27 days was selected, whereas in the present case, the position of the minimum resulting from the fit of a Gaussian curve plus a second-degree polynomial that represents the background is chosen. The resulting periods are then slightly shifted towards higher values due to the likely contribution of higher hidden periodicities present but not resolved as was shown. This fact led us to analyze in more detail the shape of the periodograms, which do show certain peculiarities.

On examining figures 5.3, 5.8, 5.9, and 5.11, it is clear that there is a systematic decrease of the coronal rotation rate from the equator up to the poleward boundary of the mid-latitude streamers, increasing again at higher latitudes (the lack of green line emission close to the poles does not allow us to extend the analysis further). Furthermore, if we consider the width of the peaks as an indicator of the possible range of periods contributing to the average (Figure 5.4), we found that at the position of the mid-latitude streamers, a departure from rigid rotation is confirmed.

As mentioned above, the time series at mid-latitudes appear to contain several periodic components. Although one main periodicity can be easily inferred from the inspection of the periodograms, it is difficult to say anything about the other periodicities through the analysis of such curves. Some other peaks can be due to spectral leakage of a main periodic component, other peaks may not be resolved if the corresponding periodicities are too close to the main one, etc. The existence of more than one peak can result either from the presence of more than one period component during the observation time, or from modulation at one period produced by active areas that grow, decay, and later reappear at different longitudes. Our analysis with synthetic data tends to support the former explanation strengthened by the fact that there were no active regions at mid-latitudes during this phase of the solar activity cycle.

It has been suggested that the topology of the magnetic field in the solar corona is not that of a simple magnetic dipole, but higher order components are present even at solar minimum (Hoeksema, 1986; Bird \& Edenhofer, 1990). The recent analytical "dipole-quadrupole-current sheet" (DQCS) coronal magnetic field model developed by Banaszkiewics et al. (1998) can help us to trace back the green line pattern that produce such rotation profile to photospheric levels (Figure 5.14). The most striking result to emerge from Figure 5.14 is that the rotation map follows the general structure of the magnetic field model as it extends in the inner solar corona. The boundary between open and closed field lines (i.e., poleward boundary of the mid-latitude streamers) is the region which seems to deviate strongest from rigid rotation and has likely more than one periodic component. This fact is supported by the analysis performed by filtering out specific periodic components, as was shown in Figure 5.11. Therefore, in view of the explanation given above, we interpret the "absorption" peaks of the periodograms obtained as follows ${ }^{6}$ :

1. The main peak at $\sim 27.2$ days below $21^{\circ}$ latitude is due mainly to the contribution of the emission above active regions which are near the equatorial belt at this phase of the solar activity cycle.

2. Beyond $21^{\circ}$ in latitude, the relative contribution to the green line emission above neutral magnetic lines in

\footnotetext{
${ }^{6}$ The values of latitude to be mentioned are referred to a solar distance of about $1.3 \mathrm{R}$.
} 
the photosphere becomes stronger, because of the negligible contribution of active regions at these latitudes during this phase of the solar activity cycle. The peak at $\sim 14$ days thus refers likely to the symmetric appearance around the solar rotation axis of the green line pattern at these latitudes, as already reported by, e.g., Schwenn et al. (1997). It suggests the presence of coronal enhancements structured in two regions approximately opposite in longitude, this fact confirming previous results of Antonucci \& Svalgaard (1974).

3. At $27^{\circ}$ latitude and above, the emission above neutral lines in the photosphere is practically the only responsible for the green line intensity at this time of the solar activity cycle. At these latitudes two different periodicities, i.e., one at $\sim 27.2$ days and the other at $\sim 30$ days, are identifiable in the emission intensity pattern. The $\sim 27.2$ days periodicity is found at every latitude, corresponding to a component exhibiting an almost rigid rotation at the equatorial rotation rate. The second one at $\sim 30$ days slightly changes with latitude, suggesting differential rotation behaviour. On the other hand, the other peak at $\sim 14$ days would

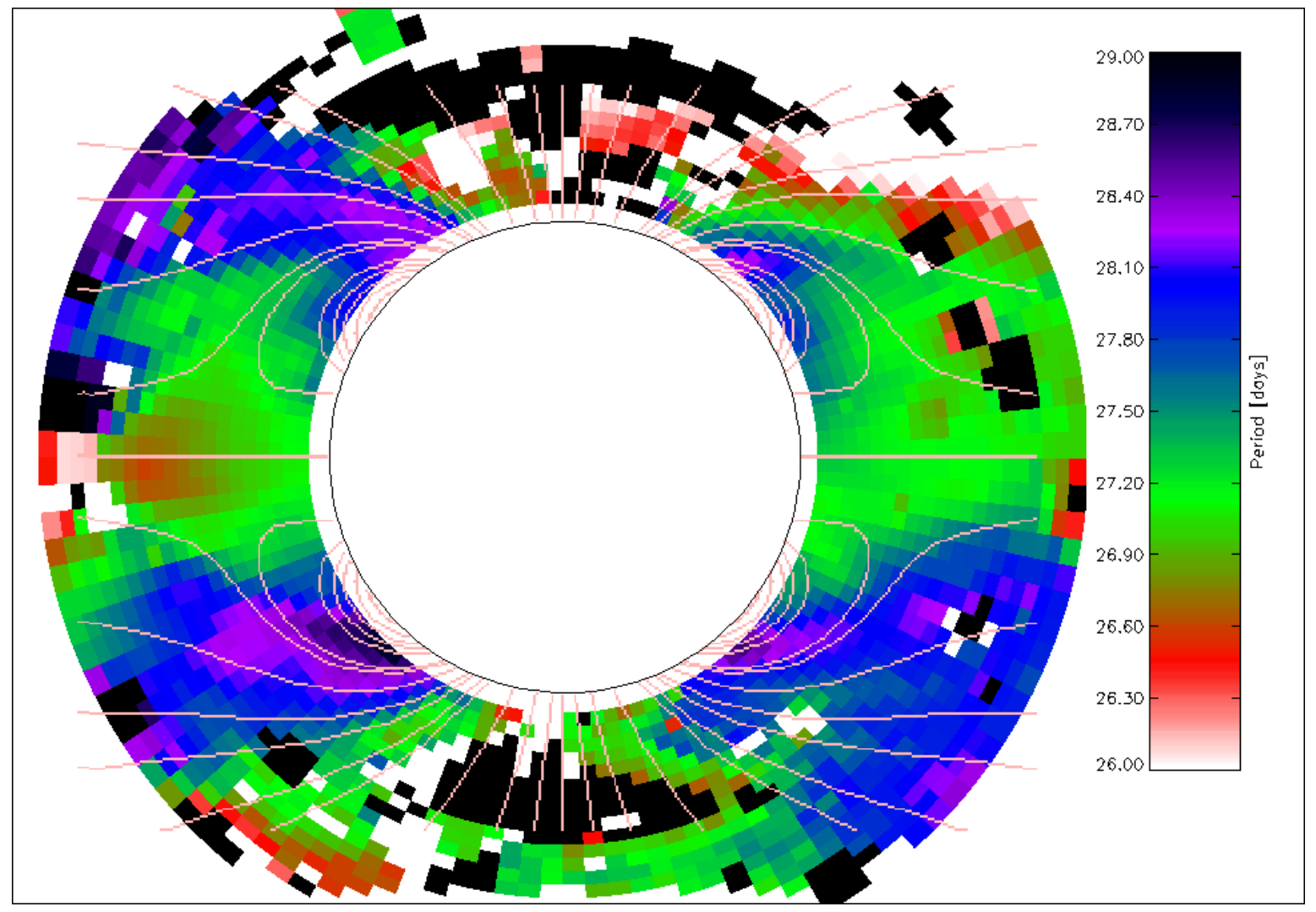

Figure 5.14: Grey-scale representation of the variation of the computed synodic rotation periods (Method $B_{1}$ ) with position angle and radial distance, superposed with the "dipole-quadrupole-current sheet" (DQCS) coronal magnetic field model developed by Banaszkiewics et al. (1998) for $Q=1.5$. Note that the spacing of the field lines shown, does not represent the field strength but is simply intended to provide a clear representation. 
be again related to the symmetry of the green line pattern, which at these latitudes merge well into the heliospheric current sheet as observed in LASCO-C2 and -C3 images, indicating that hot magnetic loops are closely associated with high density streamers on top.

4. Above $57^{\circ}$ latitude, we do not see any longer the contribution of the component exhibiting a periodicity of $\sim 30$ days, either because this component tends to diminish or the signal is too weak to allow reliable detection.

Therefore, our analysis suggests that in the green line emission corona two components (at least) rotating at different rates are identifiable, i.e., one rotating almost rigidly at the equatorial rotation rate, and the other following a differential rotation pattern. The former traces the rotation of the long-lived global-scale magnetic field whereas the latter corresponds to small scale structures. The rotation pattern we infer for the component rotating differentially resembles that obtained by Stenflo (1989) from the analysis of the recurrence of the photospheric magnetic pattern during several solar rotations.

\subsection{Part II: Analysis of the longitude displacement of coronal and transition-region features}

Our goal in this second part of the Chapter is to analyze the rotation rate with a method involving shorter timescales. Tracking of coronal features and estimate of their speed rather than analysis of their recurrence after an integer (semi-integer) number of solar rotations is the method to be employed. The optically thin character of the Fe XIV emission added to projection effects in coronagraph images do not allow a reliable estimate of the angular displacement of the coronal features. Therefore, we use disk observations of the transition region and corona at different EUV wavelengths obtained by the EIT instrument onboard SOHO.

\subsubsection{The EIT observations}

The EIT instrument onboard SOHO provides images in four wavelength ranges centered on lines of the coronal species $\mathrm{Fe}^{8+} / \mathrm{Fe}^{9+}(17.1 \mathrm{~nm})^{7}, \mathrm{Fe}^{11+}(\mathrm{Fe} \mathrm{XII}$ line at $19.5 \mathrm{~nm})$, and $\mathrm{Fe}^{14+}(\mathrm{Fe} \mathrm{XV}$ line at $28.4 \mathrm{~nm})$, and the transition region line of $\mathrm{He}^{+}(\mathrm{He}$ II line at $30.4 \mathrm{~nm}$ ). The temperatures of maximum fractional abundance are 1.0, 1.4, and $2.1 \times 10^{6} \mathrm{~K}$, respectively for the coronal species, and $\sim 80000 \mathrm{~K}$ for the $\mathrm{He}^{+}$ion. The $30.4 \mathrm{~nm}$ emission is dominated by transition region gas expected to be located in a thin layer just above the chromosphere, whereas the coronal lines arise in a range of heights throughout the corona. Hence, the instrument is well suited to provide the data necessary for our analysis. See, e.g., Figure 5.15.

The data sets used for the study of longitude displacement of coronal and transition region features are extracted from full-disk, full-resolution EIT images in each of the four bandpasses, acquired between May 10 and May

\footnotetext{
${ }^{7}$ The $17.1 \mathrm{~nm}$ band is dominated by both Fe IX and Fe X emission lines; these lines being produced by the Fe $\mathrm{F}^{+}$and Fe $\mathrm{e}^{9+}$ ions, respectively.
} 

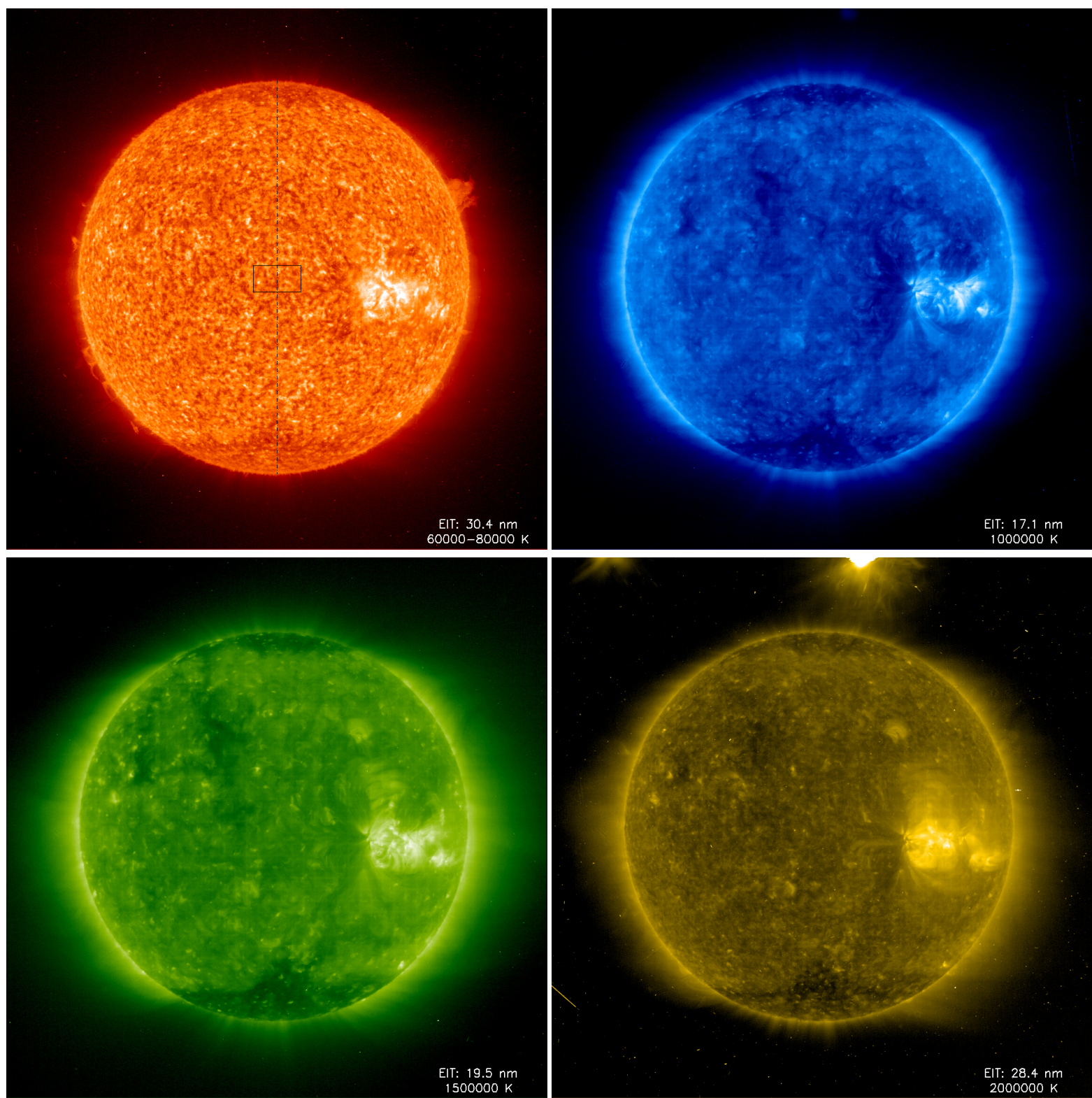

Figure 5.15: EIT images on May 16, 1996. In the upper left panel, a latitude strip to scale is shown. The images are representative at the corresponding wavelength of the structures to be tracked in their pass through the central meridian.

23, 1996. Before creating the data sets, the raw images were preprocessed. The preprocessing ${ }^{8}$ includes dark subtraction, degridding ${ }^{9}$ and flatfielding.

\footnotetext{
${ }^{8}$ The preprocessing of the EIT images was performed with the "Solar software" package provided by the SOHO consortium.

${ }^{9}$ The raw EIT images show a periodic intensity modulation pattern, referred to as a "grid pattern". It is due to a smoothed out-of-focus image of a nickel grid (Delaboudinière et al., 1995) located $14.5 \mathrm{~mm}$ in front of the CCD detector. For details see, e.g., Moses et al. (1997).
} 


\section{The data sets}

For the generation of each of the four data sets we used two to four EIT full disk images per day (depending on availability) during the 14 days period mentioned above. Thirty six latitude zones, or strips, are extracted from each image, each of them being a rectangle of equal projected area. These zones are 90 pixels ( $\sim 234$ arcsec) wide by 50 pixels $(\sim 130 \mathrm{arcsec})$ long, along the central meridian of the Sun (see, e.g., the upper right panel of Figure 5.15 and Figure 5.16). The distance between the bottom part of one strip and the bottom part of the next one is fixed to 20 pixels $(\sim 52 \operatorname{arcsec})$.
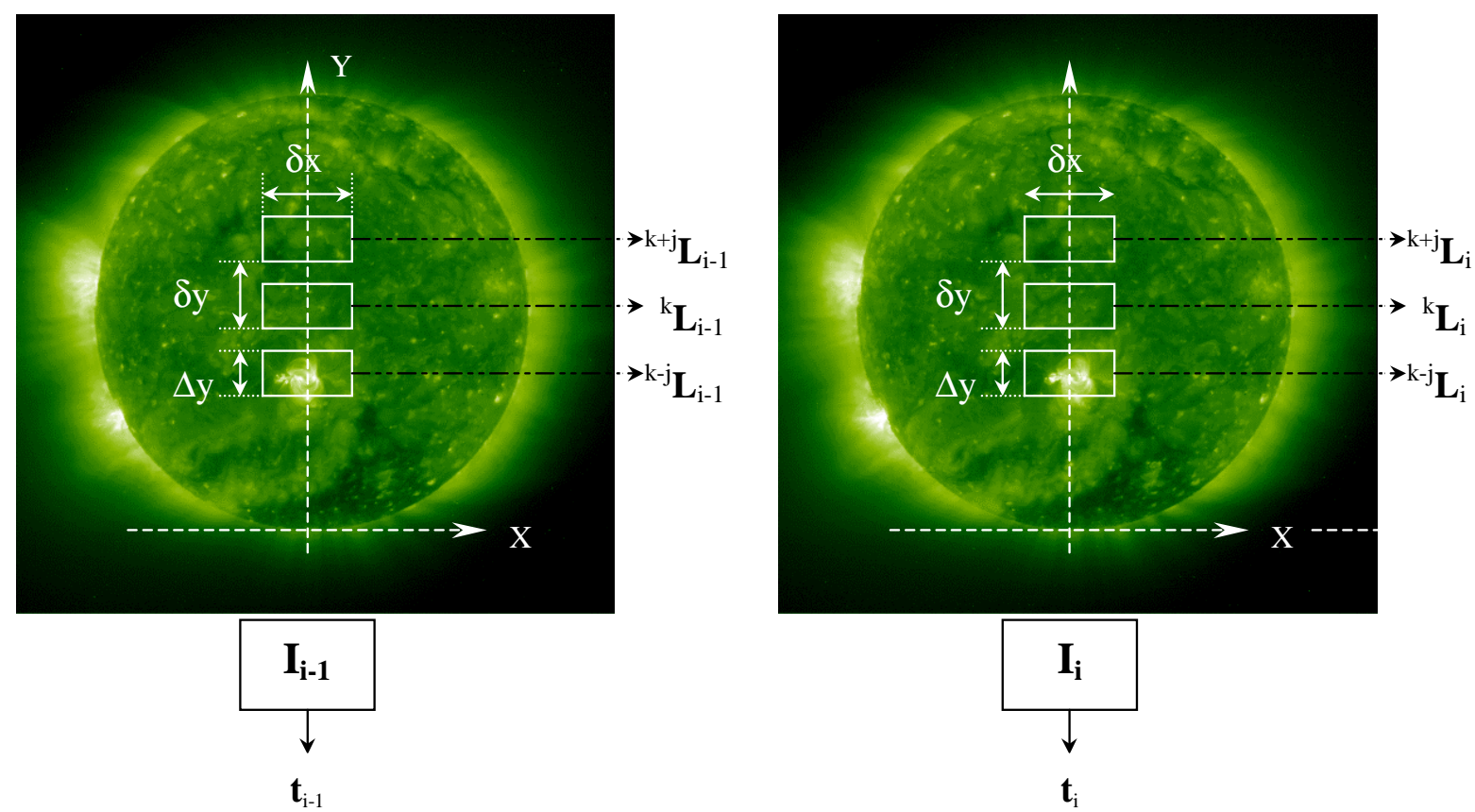

Figure 5.16: Definition of latitude strips for the determination of the longitude displacement of coronal and transition region features. The whole time interval spans 14 days, the time difference between consecutive images being between 6 and 12 hours (depending on availability). The zones drawn in the sketch are not to scale: $\Delta x=90$ pixels $(\sim 234$ arcsec); $\Delta y=50$ pixels $(\sim 130$ arcsec $) ; \delta y=20$ pixels $(\sim 52$ arcsec). For details see text.

\subsubsection{The method to compute the longitude displacement}

The determination of the rotation rate for each of the four species is computed by dividing the longitude displacement suffered by the structures within each latitude zone between consecutive images by the time lag between the corresponding images. The longitude displacement of the characteristic features is determined through crosscorrelation of the intensity pattern in each latitude zone in consecutive images.

Let us now address the description of the method. The steps of the procedure used will be explained in terms of a generic data set; the method being repeated for each of the four wavelengths mentioned. Let us define $L{ }_{i}^{k}=L\left(t_{i}, y_{k}\right)$ to be the intensity pattern in the latitude zone $k$ with its center on the central meridian at the $y_{k}$ pixel on the image 
$I_{i}$ taken at $t_{i}, \delta t=t_{i}-t_{i-1}$ the time elapsed between two consecutive images, and $\delta y=y_{k}-y_{k-1}=20 \mathrm{pxl}$ the distance in pixels between the centers of two consecutive latitude zones in the $I_{i}$ image (see Figure 5.16). Hence, the algorithm can be briefly outlined as follows:

\section{Building of the data sets}

For each of the four wavelengths, determination of rectangles of equal projected area $L_{i}^{k}$ in every image $I_{i}$ ( $i=0 \ldots N_{i} ; k=0 \ldots N_{k}$, where $N_{i}$ and $N_{k}$ are the number of images and the number of latitude zones in each image, respectively), is made.

\section{Determination of the displacement of the features in consecutive images}

a. Cross-correlation of the intensity pattern in each of the latitude zones of image $I_{i-1}$ with the corresponding one in image $I_{i}$, i.e.,

$$
X_{i}^{k}=L_{i-1}^{k} \otimes L_{i}^{k}
$$

b. The position of the maximum of the 2-dimensional array representing the resulting cross-correlation, i.e., $\max \left(X_{i}^{k}\right)=M_{i}^{k}=(x, y)$, gives the number of $(x, y)$ pixels the pattern in the $L_{i}^{k}$ strip has to be shifted in East-West (E-W) and South-North (S-N) direction, respectively, to match the pattern in the $L_{i-1}^{k}$ strip. The $x$-value reflects predominantly solar rotation and the $\mathrm{E}-\mathrm{W}$ component of proper motions of characteristic features within the latitude strip, while the $y$-value reflects the $\mathrm{S}-\mathrm{N}$ component of proper motions.

\section{Increasing of statistical significance}

The $x$-component of each individual determination of $M_{i}^{k}$ is not a good estimate for our purposes because it includes the proper motion of active features that could be present in the respective latitude strips. Therefore, in order to reduce the contribution of the proper motion (and increase the relative contribution of the general rotation), it is desirable to lower its statistical significance. The median of $M_{i}^{k}$ in the index $i$ is a good choice ${ }^{10}$, since different features are involved in each calculation of $M_{i}^{k}$. In this way, the random character of the proper motion contribution tends to cancel it (see Section 5.3.3). In other words, $M^{k}(x)=\operatorname{median}\left(M_{i}^{k}(x)\right.$ ) is a convenient estimator of the horizontal displacements due to rotation effects.

\section{Change of reference system}

Up to now, the position of the center of each latitude zone $y_{k}$ is given by the pixel number in the heliographic South-North direction of the image. This pixel number is then converted to an equivalent heliographic latitude $\varphi_{k}$. The resulting range is from $\left[-90^{\circ}, 90^{\circ}\right], 0^{\circ}$ being at the solar equator. The tilt angle $B_{0}$ of the Sun's rotational axis is accounted for in this step.

\section{Conversion of linear velocity (pixels/hour) to angular velocity (degrees/day)}

The "median" E-W shift in pixels $M_{k}(\varphi)$ obtained for each latitude zone divided by the normalized time

\footnotetext{
${ }^{10}$ The use of the median instead of the average is to minimize the effect of extreme values obtained by wrong determination of the longitude displacement.
} 
lapse in hours between consecutive images is equivalent to the linear displacement rate as function of latitude (pixels/hour). Under the approximation of small angles, the conversion to deg/day (synodic) is straightforward and given by ${ }^{11}$

$$
\omega_{s y n}(\varphi)=M\left(\varphi_{k}\right) \cdot R \cdot \zeta \cdot 24 \cdot 3600^{-1}
$$

where $R$ represents the varying Sun-Earth distance in units of $\mathrm{R}_{\odot}$ (although considered constant for the 14 days period) and $\zeta$ is the number of arcsec subtended by one pixel of the EIT instrument ( $2.6 \operatorname{arcsec})$.

\section{Determination of the rotation curve as function of latitude}

a. Plot of $\omega(\varphi)$ vs $\varphi_{k}$.

b. Weighted $\left(1 / \sigma^{2}\right)$ least square fit of the obtained data.

\subsubsection{Results}

We measured the longitude displacement of coronal and transition region features in their pass through the central meridian from a statistical point of view. The rotation rates (periods) $\omega(\varphi)$ obtained according to the method described above for the four wavelengths aforementioned are shown in the respective four panels of Figure 5.17. The individual error values, denoted in the graph by vertical lines, are given by the standard deviation of $M_{i}^{k}$ in the index $i$ (see steps 2 and 3 of Section 5.3.2). The highest dispersion of individual measurements is observed in the graph corresponding to the rotation rate obtained from $\mathrm{Fe}^{8+} / \mathrm{Fe}^{9+}$ data. The reason is found by a closer inspection to the individual images at this wavelength: they are in general less structured than the images at the other coronal wavelengths (compare, e.g, the four images of Figure 5.15).

\section{Curve fitting}

The traditional and easiest way used to present rotation profiles as obtained with different tracers is by a least square fit of them with the functional form

$$
\omega(\varphi)=A+B \sin ^{2} \varphi+C \sin ^{4} \varphi
$$

where $\omega(\varphi)$ represents the rotation profile as function of the solar latitude $\varphi$ either as rotation rate, e.g., in $\mathrm{deg} / \mathrm{days}$, or as rotation period, i.e., in days. The coefficient $A$ gives the solar rotation (rate or period) at the equator, whereas the coefficient $B$ and $C$ account for the departure of rigid rotation as function of latitude, i.e., the differential rotation.

The large scatter in the obtained data (denoted by vertical lines in the plots in Figure 5.17) suggests the use of a first order polynomial in $\sin ^{2}(\varphi)$ as fitting function to avoid the wiggles characteristic of orders greater than 1. The coefficients $A$ and $B$ obtained with their standard deviations are shown in Table 5.2 and the respective fitted curves are plotted in Figure 5.18. The row marked with an "**" corresponds to a similar analysis performed on data

\footnotetext{
${ }^{11}$ The conversion to sidereal rotation rate, i.e., $\omega_{\text {syd }}(\varphi)$, is given simply by the relation $\omega_{\text {syd }}(\varphi)=\omega_{\text {SOHO }}(\varphi)+\omega_{\text {syn }}(\varphi)$, where the angular speed of the SOHO spacecraft $\omega_{\text {SOHO }}(\varphi)$ around the Sun is simply that of the Earth, i.e., $0.9863 \mathrm{deg} / \mathrm{day}$.
} 

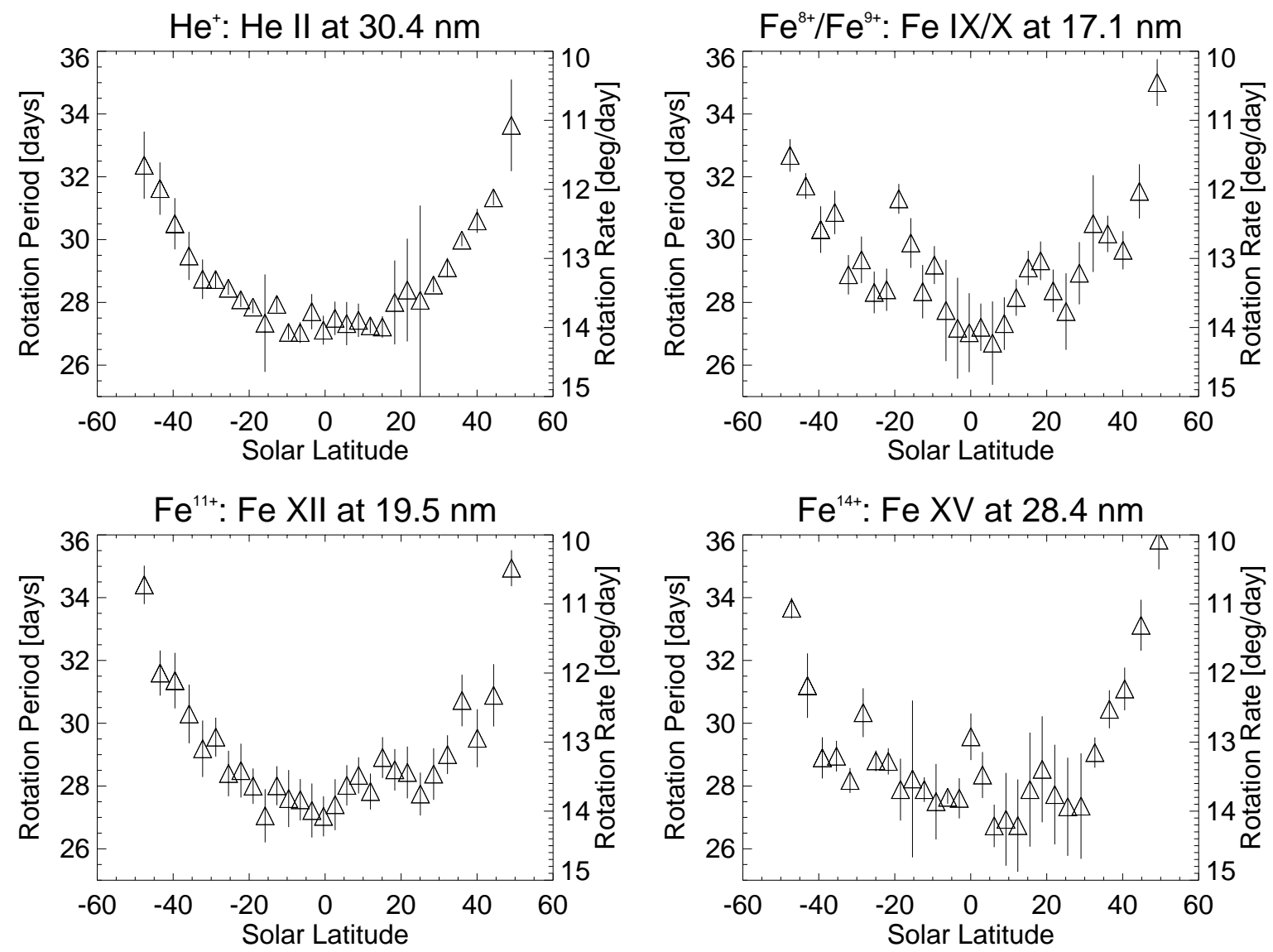

Figure 5.17: Latitude dependence of the synodic rotation rate (rotation period) profiles obtained by analyzing the longitude displacement of coronal $(17.1 \mathrm{~nm}, 19.5 \mathrm{~nm}$, and $28.4 \mathrm{~nm})$ and transition region $(30.4 \mathrm{~nm})$ features in EIT/SOHO images between May 10, and May 23, 1996. The errors, represented by the vertical bars, were calculated according to steps 2 and 3 of Section 5.3.2. For details see text.

acquired between April 1 and April 14, 1997, the coefficients obtained being shown for comparison. They were analyzed in order to test the reproducibility of our method provided the results do not depend strongly on the phase of the solar activity cycle. We found that the curves corresponding to both sets represent the same rotation profile within the error under which they are valid representations.

The rotation curves obtained by i) analysis of the longitude displacement of the magnetic field pattern of the photosphere after a given time interval (Snodgrass, 1983), and ii) observations of Doppler shifts of photospheric Fraunhofer lines (Howard et al., 1983) are also shown for comparison in Figure 5.18 in pink and blue, respectively. It can be seen that, in general, the rotation rate at latitudes beyond $\sim \pm 15^{\circ}$ of both the photospheric plasma (Howard, 1983) and the photospheric magnetic field (Snodgrass, 1983) is faster than that obtained from the analysis of EIT data. This fact would be in apparent contradiction with previous results, i.e., that higher layers of the Sun's atmosphere rotate faster (see Section 5.1). This behaviour could be attributed in part to a simple mathematical artifact, which arises from the model used to fit the data, i.e., a polynomial curve to order 1 in $\sin (\theta)$. However, 


\begin{tabular}{lccccc}
\hline \hline Line & $\lambda(\mathrm{nm})$ & $A$ (days) & $\sigma_{A}$ (days) & B (days) & $\sigma_{B}$ (days) \\
\hline He II & 30.4 & 26.94 & 0.13 & 8.25 & 0.51 \\
Fe IX/X & 17.1 & 26.91 & 0.65 & 9.82 & 2.24 \\
Fe XII & 19.5 & 27.19 & 0.59 & 9.30 & 2.27 \\
Fe XV & 28.4 & 26.65 & 0.25 & 10.95 & 0.90 \\
Fe XII $\left(^{*}\right)$ & 19.5 & 27.25 & 0.50 & 8.80 & 2.12 \\
\hline \hline
\end{tabular}

Table 5.2: Least square fitting coefficients $A$ and $B$ (5.2) with their rms $\sigma$ scatter. The row marked with an "*” corresponds to $\mathrm{Fe}^{11+}$ EIT data taken between April 1 and April 14, 1997, whereas the first four rows correspond to EIT data from May 10 to May 23, 1996.
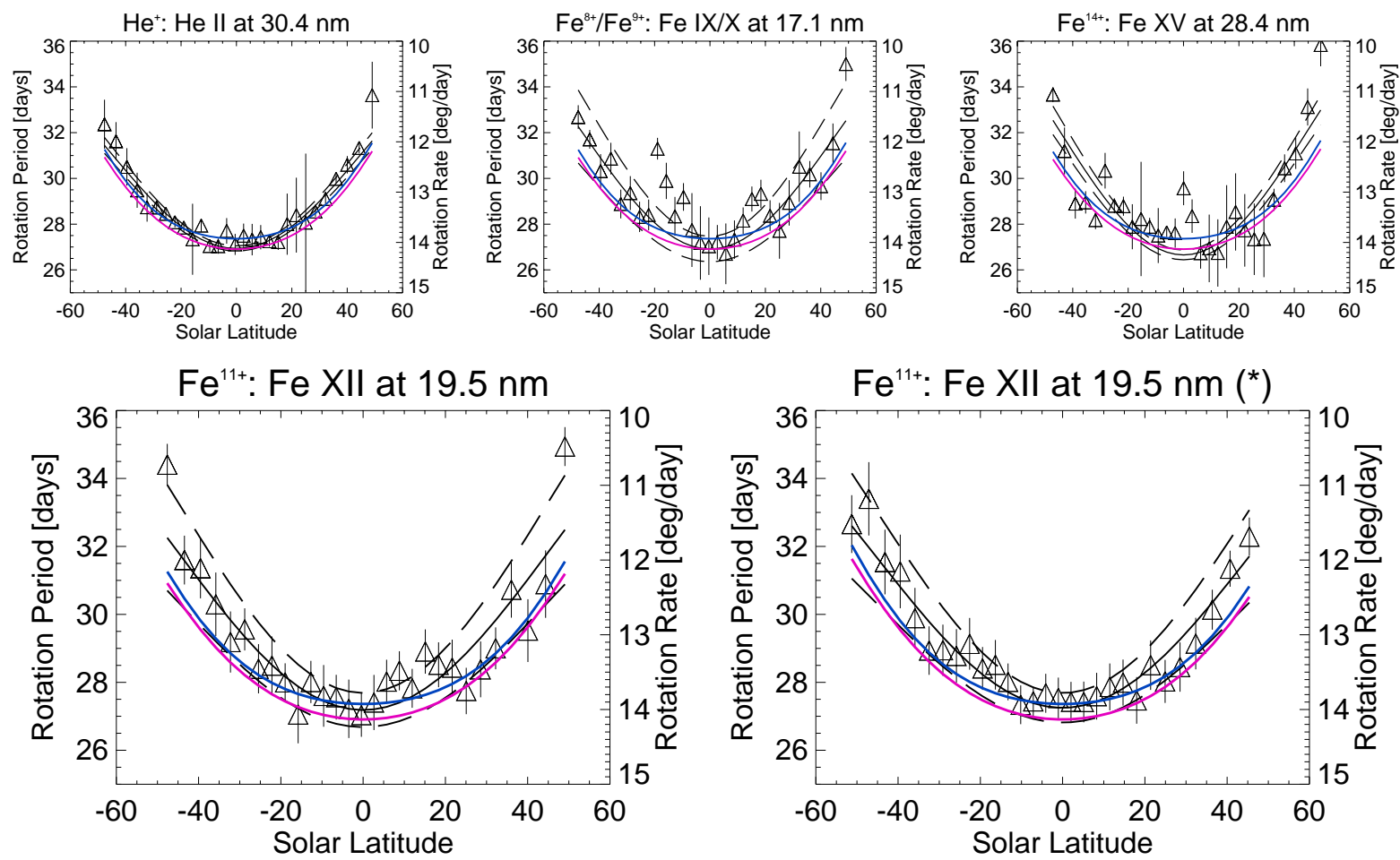

Figure 5.18: Synodic rotation period (rotation rate) as obtained from EIT data vs solar latitude. The graph marked with an “*” corresponds to $\mathrm{Fe}^{11+}$ EIT data taken between April 1 and April 14, 1997, whereas the rest of the graphs corresponds to values calculated from EIT images between May 10 and May 23, 1996 (same as Figure 5.17). The solid curves are the result of a least square fit of the data with the model given by Equation 5.2 to order 1. The dashed lines delimit the region where the fitted curves are valid. They were computed using the coefficients and standard deviations given in Table 5.2. For comparison, the rotation rates calculated by Snodgrass (1983) and Howard (1983) are plotted in pink and blue, respectively. For details see text.

a trend toward faster rotation at mid-latitudes can be discerned from the plot of the data used to determine such 
curves. Therefore, the high dispersion of the rotation rates obtained at different latitudes added to the trend aforementioned suggest that analysis of the distribution of the individual measurements be carried out, in order to give the right interpretation to our results. Such an analysis is outlined in the following section.

\subsubsection{Discussion}

Before beginning the discussion of our results, it is instructive devise a Gedankenexperiment. It consists of applying the method to a "synthetic scenario", in order to better understand the results obtained. In the method we employed, we "take photos" through a fixed window of a changing scenario. The scenario consists of objects that cross the field of view of our hypothetical camera at certain speeds. The speed of the objects in the scene are calculated by studying the horizontal displacement of the "most visible" object(s) between two consecutive photos. It is clear that many different objects will cross the field of view of our camera during the observation interval. Let us first think of a class of objects with equal speeds. In this case, one will measure the same speed in each pair of photos. Therefore, the probability distribution of the values obtained with each pair of images should have a $\delta$-peak at the position of the constant speed of the objects. On the other hand, if in our hypothetical scene the objects crossing the field of view move with random speeds, the distribution will be "flat", spanning all the possible values of speed. In a case with two distinct classes of objects, one class moving at speed $v_{1}$ and the other at speed $v_{2}$, the probability distribution will have two main peaks centered at the respective velocities.

Coming back now to our object of study, the Sun, the scenario our camera observes is a region of a certain layer at the central meridian of the Sun. The objects are the typical structures seen at each wavelength, i.e., mainly the transition region network and active region enhancements at $30.4 \mathrm{~nm}$, and bright points and active regions embedded in a diffuse background in the case of coronal images.

The implicit condition assumed to consider the median of the respective measurements as a valid estimate of the longitude displacement, is that all the structures that contribute to the estimation of the displacement move at roughly the same speed around a mean value characteristic of that latitude bin. In other words, the rotation speed at that latitude is more or less the same for all the structures crossing the central meridian during the fourteen days the time series spans. According to our Gedankenexperiment, the normalized histogram of the measurements corresponding to each latitude bin should exhibit a main peak at the corresponding rotation speed (period), with a dispersion characteristic of the statistical nature of the measuring process. However, inspection of the distribution of the points, i.e, normalized histograms (figures 5.19 and 5.20), shows that this is not the case, at least for the Fe XII emission. The appearance of more than one peak in some of the histograms is a common feature at middle latitudes. That tells us that the objects caught by our camera for a given latitude bin may be grouped according to their speeds (rotation period). Unfortunately, at the other wavelengths the number of images used is less and not enough for a reliable statistics. In the Fe XII case, it is justified by the similar rotation pattern found for both sets, both sets comprising a different number of images taken almost one year apart. Further, comparison of histograms of both Fe XII sets at similar latitudes yields to the same kind of interpretation for both sets (compare, e.g., the histograms of Figure 5.20 with the respective ones of Figure 5.19).

Therefore, the distribution of the measurements shown in figures 5.19 and 5.20, suggests the coexistence at 

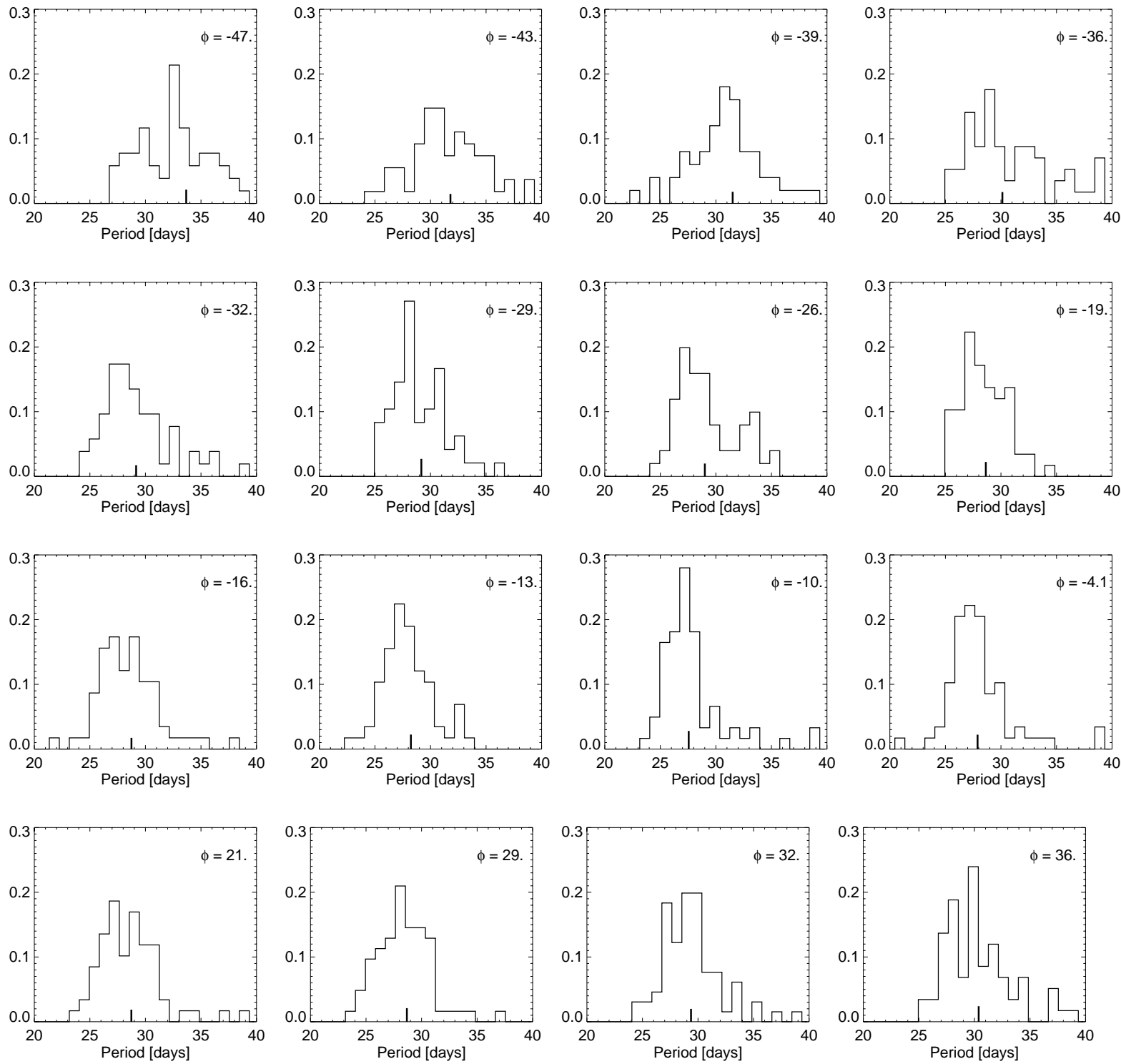

Figure 5.19: Distribution of the measurements that give place to the median value representative of the rotation rate (small vertical thick line) at the latitude $\phi$ for the $\mathrm{Fe}^{11+}$ data set obtained between April 1 and April 14, 1997. The area under the histograms is equal to 1 .

the same latitude bin of features rotating at different speeds. In this view, the corresponding rotation periods are close either to that corresponding to the equatorial rotation rate or to that corresponding to the footpoints where the structures detected by the method are anchored. The latter follows more likely the differential rotation pattern.

The use of the median value as the estimate of the rotation period gives a value that matches that corresponding to the photospheric rotation period within the error of the method, as it was shown in Figure 5.18. However, its use smears out the coexistence of different emission patterns rotating at different speeds at a given latitude, as 

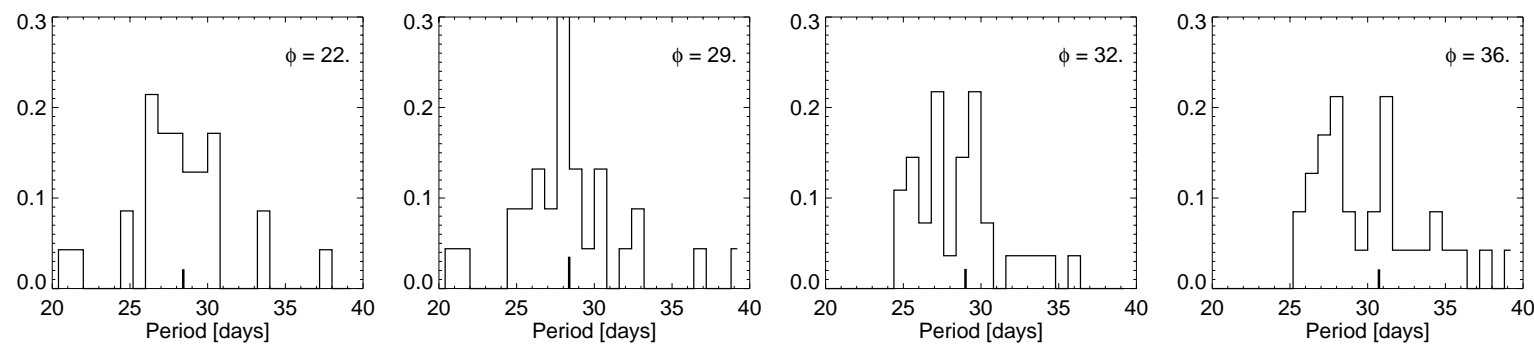

Figure 5.20: Distribution of the measurements that give place to the median value representative of the rotation rate (small vertical thick line) at the latitude $\phi$ for the $\mathrm{Fe}^{11+}$ data set obtained between May 10 and May 23, 1996. The area under the histograms is equal to 1 .

suggested by figures 5.19 and 5.20. Therefore, this analysis supports the interpretation drawn by the analysis of LASCO-C1 green line data at longer time scales, i.e., the coexistence at a given latitude of emission patterns rotating at different speeds.

\subsection{Concluding remarks}

Briefly, we summarize the steps we performed for the analysis of the green line coronal data (Section 5.2). First, by selecting the position of the minimum as the correct period (methods A and B in Paper I), we found a quasirigid rotation law, confirming the results of Sime et al. (1989). By fitting the broad Gaussian-like component of the periodograms with a linear combination of a Gaussian and a second-degree polynomial to represent the background, we found the rotation rate to be slightly faster than that found in Paper I, at the position of the midlatitude streamers (methods $\mathrm{B}_{0}, \mathrm{~B}_{01}$, and $\mathrm{B}_{1}$ in Section 5.2.3). We attribute this fact to the contribution of secondary peaks at higher periods, as it would be expected if several rotating components coexist at the same spatial location. Finally, when analyzing the detailed structure of the periodograms as function of latitude and radial distance by filtering out specific periodicities, the co-existence of more than one periodic component at the position of the mid-latitude streamers is revealed. Moreover, the analysis of the longitude displacement of structures crossing the central meridian in the EIT Fe XII images (Section 5.3.4) suggests the coexistence of features moving at different speeds at middle latitudes. Extrapolated to longer time scales, i.e., more than one solar rotation, it further supports the interpretation given to the analysis made on LASCO-C1 data, i.e., the existence at a given projected latitude of at least two periodic components in the emission intensity pattern.

The green line mid-latitude streamers converge into a single-flat near-equatorial current sheet as seen in white light, where the slow solar wind is observed to flow. If the rotation trend remains further in the heliosphere, one would expect to find the differential rotation signatures in the heliospheric current sheet (at times of solar minimum). In addition, streamers and interfaces between streamers have been regarded as most plausible sources of slow solar wind (see, e.g., Schwenn, 1983; Withbroe et al., 1991). Therefore, knowledge of the distribution of the rotation rate at small scales in the corona might thus help finding the origin and understanding the acceleration 
mechanisms of the slow solar wind. Furthermore, it can provide new constraints to the existing coronal expansion models. 



\section{Chapter 6}

\section{Summary and Outlook}

A low beta plasma as that of the inner solar corona is closely tied to the lines of force of the magnetic field. Therefore, the analysis of coronal plasma structures gives an important insight regarding direction and topology of the solar magnetic field lines. Systematic observations of the evolution of structures in the corona tell us about the solar activity in general and also about dynamic processes in all parts of the magnetically dominated corona. Since the resistivity of the plasma, specially at small scales, plays an important role and has to be taken into account in the models, observations with both high temporal and spatial resolution can provide constraints to proposed models.

In the past, observations of the solar corona have been made either during the short instances of total solar eclipses or with space- and ground-based coronagraphs. The externally occulted coronagraphs used so far in space missions suffered from vignetting at the inner edge of the occulter and did not allow useful observations inside about $2 \mathrm{R}_{\odot}$. The use of mirror optics in internally occulted coronagraphs finally overcame this problem. LASCOC1 (Large Angle Spectroscopic Coronagraph) onboard SOHO (Solar and Heliospheric Observatory) and a prior prototype PICO (Pic du Midi Coronagraph) were the first internally occulted coronagraphs designed with reflective optics. Following this line, the MICA telescope (Mirror Coronagraph for Argentina) incorporates all the advantages of internally occulting and mirror design such as compactness, low level of instrumental scattered light and optical achromacy, to name the most important ones.

MICA is a ground-based solar telescope, installed in the Prof. Ulrico Sesco Observing Station of OAFA at El Leoncito $\left(69.3^{\circ} \mathrm{W}, 31.8^{\circ} \mathrm{S}\right)$, San Juan, Argentina, at an altitude of $2400 \mathrm{~m}, 50 \mathrm{~km}$ eastwards from the Argentinean "Cordillera de los Andes". It is designed to obtain filtergrams of the inner corona ( $1.05 \mathrm{R}_{\odot}$ to $\left.2.0 \mathrm{R}_{\odot}\right)$ in the emission of the Fe XIV (530.3 nm) and Fe X (637.4 nm) coronal lines.

The essential questions that can be addressed by MICA, in conjunction with several other instruments both on ground and in space, are:

- How is the corona being heated?

- Where and how is the solar wind accelerated?

- What causes coronal transients, and what role do they play in the evolutionary development of large-scale coronal patterns?

These are the most important and long-standing problems in all coronal physics, and MICA can make significant contributions to find their solutions. Towards this goal, MICA contributes in the study of

- the global distributions of key plasma parameters and their evolution with time,

- the spatial structure of the corona on both fine and large scale, and their timely evolutions, 
- the processes that occur in coronal transients and the conditions that trigger them.

This thesis dealt with various tasks that make the MICA telescope fully operational (Chapter 2). Main emphasis was put on calibration issues (Chapter 3). Based on this detailed preparatory work, the analysis and interpretation of the features observed in MICA images could be performed (Chapter 4). In addition, the analysis of the rotation characteristics of the inner corona (Chapter 5) was performed. For such an analysis, observations from both LASCO-C1 and EIT instruments onboard SOHO were used.

In order for the MICA telescope to work in the most efficient way, a dedicated software package had to be developed (Section 2.6). Thus, a stand-alone operation of the telescope became possible. In particular, the feedback of internal data allowed the self-correction of wrong values. Furthermore, values of sky and Sun brightness constantly recorded by two auxiliary devices are used by the program to check whether the instrument must stop or can resume operation. Two and a half years of continuous operation have shown the reliability of the system.

For quantitative evaluation of the data careful calibration of the images relative to a well-known calibrated source is required. The intensity of the continuum at the center of the solar disk is used for such purposes. Further, the effect of the sky variability in the calibration must be well understood and quantified. The mathematical formulation of the calibration procedure was outlined in Chapter 3. The calibration procedure developed is actually carried out almost in real time by a program especially made for that purpose. Hence, the "almost real-time" state of the corona can nowadays be monitored at the site while the telescope records the images. The validity of the calibration procedure was tested by comparing calibrated green line images from MICA with green line scans made with the Sacramento Peak coronagraph. The comparison showed that, within the experimental error, the calibrated MICA intensities match well with those obtained with the Sacramento Peak coronagraph.

Having obtained calibrated images, the different features observed in the images can be studied and interpreted. In Chapter 4, I first discussed the possible scenarios from a mathematical point of view in order to set a common framework for the analysis of the images. This mathematical frame was then used to characterize different phenomena occurring in the field of view of the MICA telescope. Some examples were presented and described. They comprised the analysis of stationary features, and dynamical events, i.e., green line coronal transients. In the latter case, an extensive morphological description of a particular event was given. Images of the $\mathrm{H}_{\alpha}$ telescope HASTA $\left(\mathrm{H}_{\alpha}\right.$ Solar Telescope for Argentina), installed also at El Leoncito, were used to help in the analysis. The use of simultaneous observations at two wavelengths representative of very different temperature regimes helped to track the temperature evolution of the coronal plasma during the dynamical evolution of the event. The analysis of this dynamical event emphasized the importance of multiwavelength observations of the solar corona as close to the limb as possible and with the highest time resolution. The interpretation drawn from the analysis suggests that the existence of particular features in the images (small local dark or bright points) indicates the position where magnetic reconnection is likely to occur.

The analysis and interpretation of coronal data was extended to studying the coronal emission pattern on longer time scales. In particular, the recurrence of the green line emission pattern was investigated. Data from the LASCO$\mathrm{C} 1$ instrument were used for such a task (Section 5.2). Detailed analysis of the shape of the periodograms led to the conclusion that there exist at least two superposed rotating patterns at any given latitude. One of them was found to 
be independent of latitude, i.e., a rigid rotating pattern, with a rotation period that matches well that of the synodic rotation period at equatorial latitudes, i.e., $\sim 27.2$ days. The other one was found to follow differential rotation resembling that found at photospheric levels from the analysis of the recurrence of the photospheric magnetic pattern during several solar rotations. The former traces the rotation of the long-lived global-scale magnetic field whereas the latter corresponds to small scale structures with shorter life times. A similar result was reached by the study of the longitude displacement of structures crossing the central meridian on Fe XII EIT images (Section 5.3): at certain latitudes we found evidence of the existence of different features moving at different speeds. In this respect, it is important to extend the study performed on Fe XII EIT data to longer time series, in order to increase the statistical significance of such an analysis. Furthermore, this analysis should be extended to the other EIT wavelengths in order to determine the distribution of the rotation rate determinations at other altitudes. This would help to a better understanding of the temperature dependence of the coronal rotation rate. As an extension of the present study of the green line coronal emission pattern on longer time scales, it may be useful to study the recurrence of the pattern with increasing solar activity, in order to understand the variations of the coronal rotation rate with the solar activity cycle.

\subsection{Future prospects}

The extended solar corona has been recorded with unprecedented sensitivity by the LASCO-C1 coronagraph onboard SOHO. For four and a half years, it has been imaging the inner corona from 1.1 to 3.0 solar radii. In order to gain better understanding of the mechanisms involved, it is important to make observations at i) lower heights (where the core of prominences lies and where flares likely originate), ii) higher time cadence, since fast events are often missed during the time gap between images, and iii) to obtain simultaneous and co-ordinated data at different wavelengths. Although ground-based observations of the solar corona are strongly affected by sky conditions, they can complement space-based observations. In this line, this dissertation showed that the study of the solar corona by the MICA telescope is indeed possible and useful, despite the effects of the terrestrial atmosphere. It is thus worth the effort especially with regard to the higher time resolution achieved.

The study of dynamical events at different temperatures and heights in the solar atmosphere makes it possible to explore the relationships between, e.g., flares and eruptive prominences. MICA images are well complemented with the images from the solar $\mathrm{H}_{\alpha}$ telescope HASTA. With both instruments, the correspondence between the hot coronal pattern and the respective $\mathrm{H}_{\alpha}$ features close to the limb can be addressed. Further, the dynamic behaviour of hot and cool structures can be very well traced with both instruments. As a result, $\mathrm{H}_{\alpha}$ events can be correlated with green line events at nearly the same time, shedding new light on the long-standing issue of the causal relations between the various phenomena occurring in the solar atmosphere. Whether the triggering of eruptive activity is due to magnetic reconnection or not, is another question that the joint observations at El Leoncito are addressing.

The global change of the Earth's atmosphere due to pollution is certainly one of the major issues mankind should be concerned about. Pollution by dust particles, chemicals, and humidity changes the atmosphere's transparency for both the incoming solar spectrum and the reradiated energy. The relative roles of man-made pollution 
(e.g., from fires, agriculture, chemical industry, cars) and natural pollution (e.g., from volcanoes, meteorite impacts, forest fires) are still far from understood. Careful monitoring of the status of our atmosphere all around the globe is essential for progress in environmental research. In this context, MICA can also make an important contribution. The instrumentation allows an extremely sensitive and accurate determination of the sky transparency and the scattered light levels. Amount and spectral distribution of the sunlight scattered in the sky above the observatory are sensitive indicators of the amount and type of atmospheric pollution. Therefore, MICA measurements of the sky brightness are taken on a routine basis, every day in all four seasons. Close collaboration with atmospheric scientists of the various disciplines is intended in order to achieve a global understanding.

The automated MICA coronagraph is presently capable of recording images in the Fe XIV emission line with good temporal and spatial resolution. However, the same coronagraph could be used to record images with other filters, too. There is a provision to introduce new filters in the optical system of the MICA coronagraph. It is intended to upgrade the MICA coronagraph to observe the corona in selected wavelengths of importance in the near infrared range by introducing appropriate filters and replacing the actual CCD camera by a new one with more sensitivity in the near infrared spectral range. In particular, the line pair at $1079.8 \mathrm{~nm}$ and $1974.7 \mathrm{~nm}$ from Fe ${ }^{12+}$ at coronal temperatures around $1.4 \times 10^{5} \mathrm{~K}$ is well suited for coronal observations. The intensity ratio of these two lines allows a very sensitive determination of electron densities (Noëns et al., 1984). The line at $1074.7 \mathrm{~nm}$ belongs to the group of forbidden lines, the polarization of which is characteristic of the magnetic topology of the corona (see, e.g., Arnaud \& Newkirk, 1987 and references therein). Therefore, a set of polarizer filters would allow to determine the polarization status within the full field of view. One of the reasons to extend the capabilities of MICA to the infrared range is the reduced sky brightness in the near infrared (by a factor of 50 compared to the $500 \mathrm{~nm}$ range). Furthermore, these lines have much higher polarization sensitivity than the visible lines. The only reason these lines have not been used much in previous instruments was the difficulty in finding appropriate detectors.

Finally, I would like to point out that the intention of the German-Argentinean Solar Observatory is to provide MICA and HASTA data to the scientific community as well as to collaborate with other ground- and space-based instruments in joint campaigns. The scientists are welcome and encouraged to participate in the analysis of HASTA and MICA data in collaboration with the institutions involved. Our goal, which we expect to achieve in the near future, is to have access to summary data in almost real time once the Internet connection to the site becomes available. 


\section{Appendix A}

\section{On the Spectral Distribution of the Sunlight}

The spectral distribution of the Sun's radiation as observed from Earth's orbit is shown in Figure A.1. This curve of the flux above the Earth's atmosphere at the mean Sun-Earth distance of $1 \mathrm{AU}$, is referred to as the solar spectral irradiance. Its integral over wavelength constitutes the total solar irradiance or solar constant. It can be seen that it lies close to the curve calculated for a $5760 \mathrm{~K}$ black body, at wavelengths redward of about $0.5 \mu \mathrm{m}$. In the blue and violet, i.e., at $\lambda \leq 0.4 \mu \mathrm{m}$, the solar flux is significantly lower than the Planck curve at $5760 \mathrm{~K}$ would predict. This reduction in the Sun's flux in the blue is caused by the line blanketing effect of increasing numbers of closely packed Fraunhofer absorption lines. Its effect is to increase the mean opacity of the atmosphere at those wavelengths. Further in the violet, the continuum opacity also increases, at wavelengths sufficient to ionize the abundant neutral metals such as magnesium and aluminum.

In the ultraviolet, the character of the solar spectrum changes from absorption lines superposed on a continuum at $\lambda \geq 200 \mathrm{~nm}$ to a spectrum dominated by strong emission lines at $\lambda \geq 150 \mathrm{~nm}$, as illustrated in Figure A.2. The reason for this change is that the continuum intensity expected from a black body at photospheric temperatures

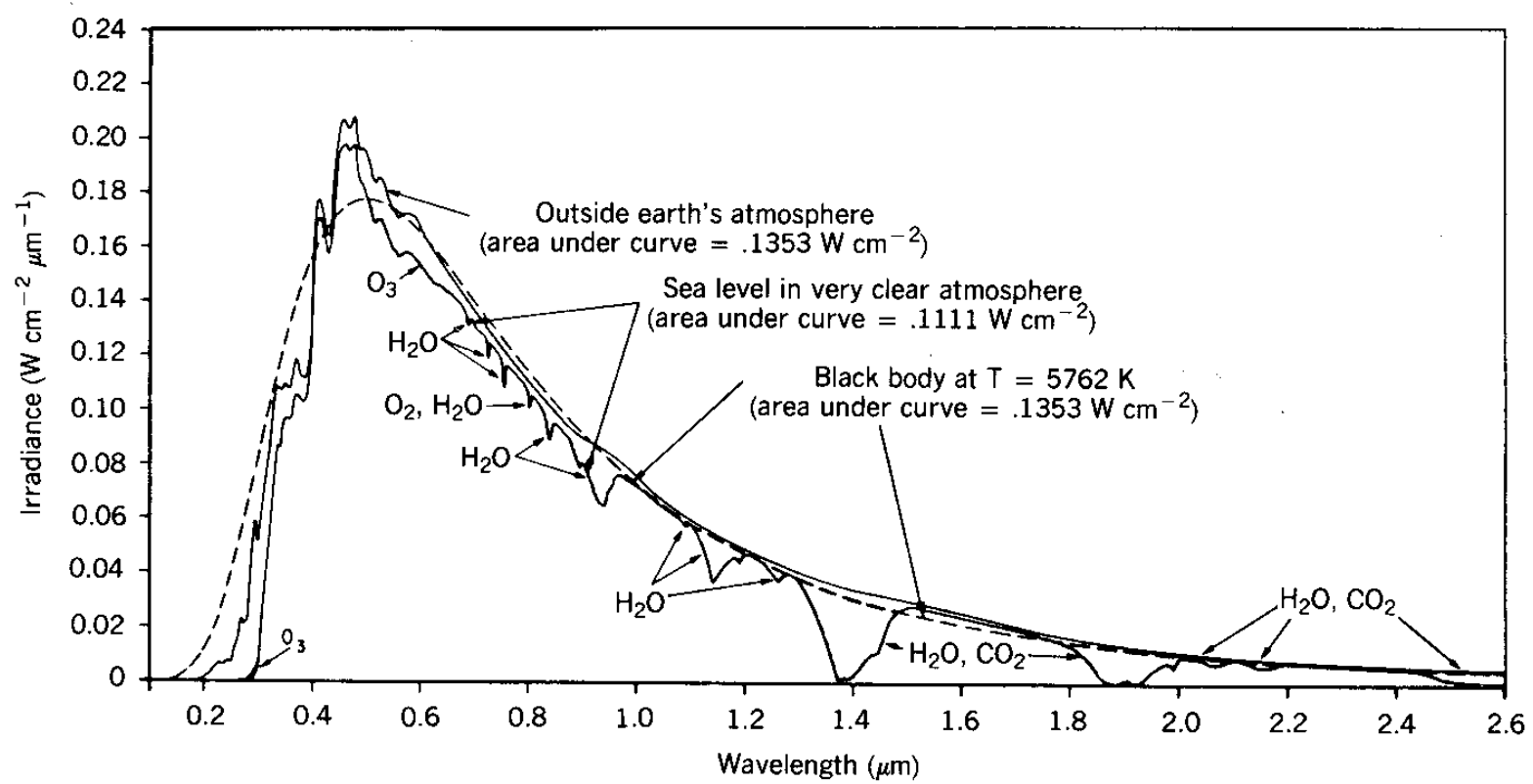

Figure A.1: Solar spectral irradiance at ground level and in space, shown together with the spectral irradiance of a black body at $5760 \mathrm{~K}$. 
follows a rapid exponential decrease from the peak near $0.45 \mu \mathrm{m}$ into the ultraviolet. The lines go into emission relative to this weak continuum, because their opacity is much greater, so we see the more intense radiation of higher and hotter chromospheric layers above the temperature minimum.

On the other hand, in the infrared, the spectrum remains a continuum with dark absorption lines, but their number decreases rapidly beyond about $1 \mu \mathrm{m}$. For details the reader is referred to, e.g., Foukal (1990).

Figure A.2: The solar ultraviolet spectrum between approximately $1400 \AA$ and $280 \AA$ obtained in 1969 by the Harvard spectrometer on the OSO 6 satellite (Huber et al., 1973). The upper spectrum in each of the 3 graphs corresponds to an active region, the lower refers to the quiet Sun. The calibration curves are shown in photons $\mathrm{cm}^{-2} \mathrm{~s}^{-1}$ sterad ${ }^{-1}$ (dashed lines) and ergs $\mathrm{cm}^{-2} \mathrm{~s}^{-1}$ sterad $^{-1}$ (solid lines). From Dupree et al. (1973). 

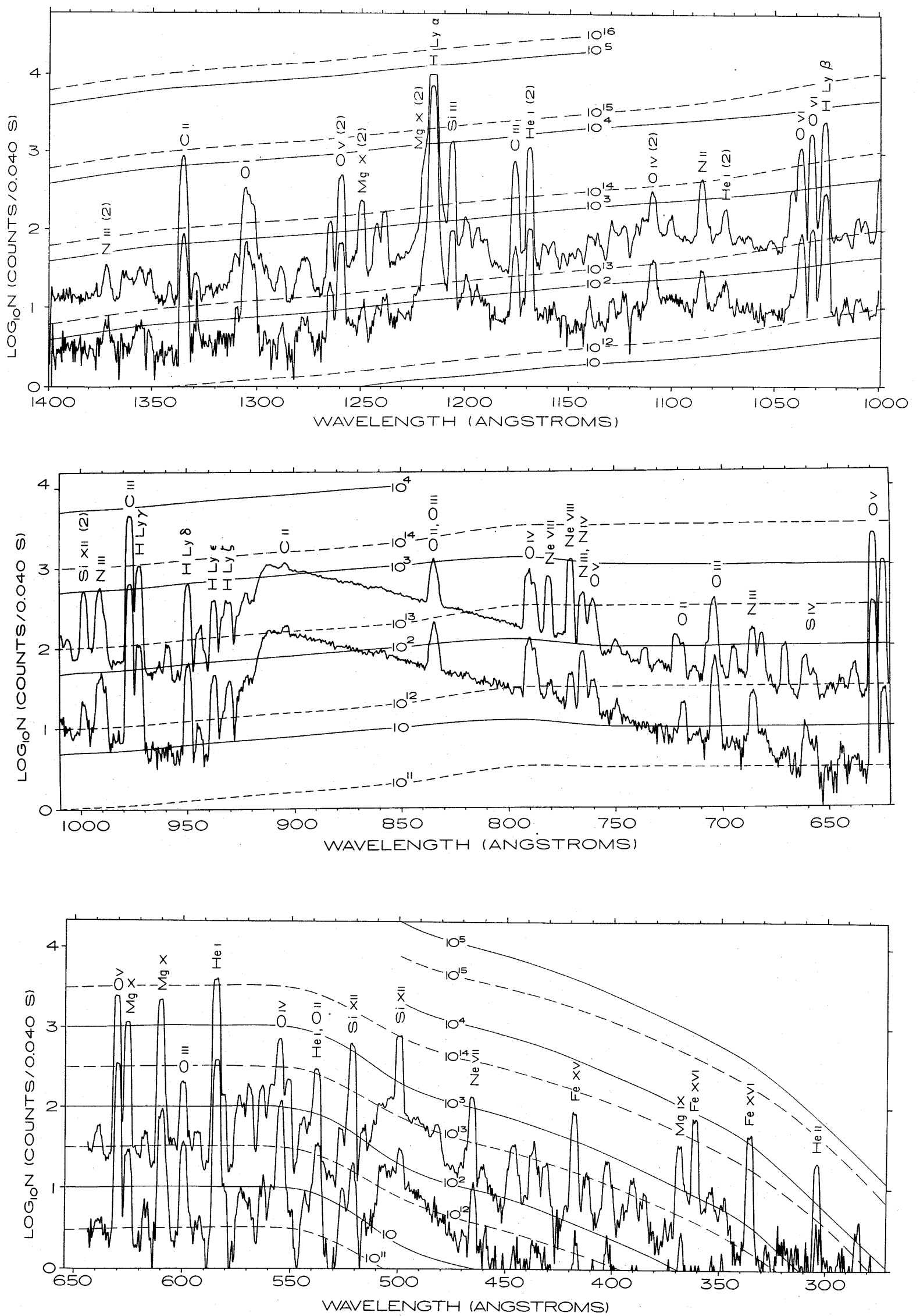


\section{Appendix B}

\section{On the Atmospheric Extinction}

The solar radiation reaching an observer on the ground is only a portion of the solar radiation entering the Earth's atmosphere: a portion is absorbed, a portion is scattered. The scattered part is partly diffused back to space, while the remainder reaches the ground as sky radiation.

Absorption of radiant energy by an atmospheric molecule is a destructive process: a photon is annihilated and its energy is transferred to the absorbing molecule which undergoes a transition from a lower to a higher energy state. Absorption may lead to subsequent emission and also can lead to the destruction of the molecule.

Scattering involves the collision of a photon with a particle, with the consequent change in the direction of motion and of the energy content of the photon and of the particle. It is caused by particles of different sizes, i.e., air molecules $\left(10^{-4} \mu \mathrm{m}\right)$, haze particles $\left(10^{-2}\right.$ to $\left.1 \mu \mathrm{m}\right)$, fog and cloud droplets (1 to $\left.10 \mu \mathrm{m}\right)$ and dust grains. When the particle is much smaller than the wavelength of the incident radiation (air molecules) the scattering can be easily treated by means of Rayleigh's theory of scattering. When the particle size is of the order of the wavelength of the incident radiation, the solution of the wave equation becomes formidable. The Mie's theory (Mie, 1908; Born, 1985) deals with this case. Under this theory Rayleigh scattering can be considered as a particular case.

For the mathematical treatment, a convenient parameter to express the size of a scattering particle is $D \pi / \lambda$ where $D$ is the particle diameter in micrometers. Let $n$ be the index of refraction and $\lambda$ the wavelength in micrometers. Then, when

- $D \pi / \lambda<0.6 / n$, scattering is governed by Rayleigh's theory. In a cloudless atmosphere, it applies to air molecules.

- $0.6<D \pi / \lambda<5$, scattering is governed by Mie's theory. It applies to particles of size greater than $10 \AA$, such as aerosols, i.e., any small solid or liquid particle suspended in the air that follows the motion of the air within certain broad limits (Iqbal, 1983).

- $D \pi / \lambda>5 / n$, scattering is a diffuse reflection process seldom occurring in the Earth's atmosphere.

\section{B.1 Air mass}

The air mass $X$ is defined as the number of times one has, along the line of sight, the quantity of air seen in the direction of the zenith (at sea level). $X$ depends on the path of the light ray, which at any altitude $h$ is determined by the direction of the incoming light $z, z$ being the angle between the light ray and the vertical ( $z$ is usually referred to as the zenith distance of the direction of the incoming light). Hence, $X(z)=X_{h} \cdot \sec z=\exp \left(h / h_{r}\right) \cdot \sec z$ denotes the air mass as a function of the apparent zenith angle $z$ at the observer's level $h$. Here $h_{r}$ denotes the vertical extent of the homogeneous Rayleigh atmosphere $(\sim 8 \mathrm{~km})$. 
It is important to have in mind that at large zenith distances, the curvature of the atmosphere, the atmospheric refraction and, the variation of atmospheric density with height complicate the exact calculation of the air mass. As a formula using true zenith distance $z_{t}$, Young \& Irvine (1967) proposed

$$
X\left(z_{t}\right)=\sec z_{t}\left(1-0.0012\left(\sec ^{2} z_{t}-1\right)\right)
$$

so $X=X_{h} \cdot X\left(z_{t}\right)$

\section{B.2 Transmittance of the Earth's atmosphere}

The transmittance or transmission coefficient of the Earth's atmosphere is defined as the ratio of radiation emerging from a medium $\left(I_{\lambda}\right)$ to incident radiation $\left(I_{0 \lambda}\right)$. The monochromatic transmittance due to direct radiation can be written as

$$
\tau_{\lambda}=\frac{I_{\lambda}}{I_{0 \lambda}}=\prod \tau_{j \lambda}
$$

where $\tau_{j \lambda}$ is the transmittance due to a single process $j$. This is given by

$$
\tau_{j \lambda}=e^{-k_{j \lambda} X_{j}}
$$

where $k_{j \lambda}$ is the monochromatic extinction or attenuation coefficient and $X_{j}$ is the optical path length. The subindex $j$ in the monochromatic extinction coefficient $k_{j \lambda}$ denotes contributions from various processes. Therefore, $k_{j \lambda}$ varies with the composition of the atmosphere. Absorption by atoms and molecules occur in limited spectral ranges, while scattering by molecules and by aerosols in suspension in the atmosphere is responsible for the continuous extinction.

\section{B.2.1 Rayleigh scattering of air molecules}

It is based on the assumption that the scattering particles are spherical, less than $0.2 \lambda$ in diameter, and that the particles scatter independently of one another. It includes considerations such as the number of particles per unit volume and the refractive index of the particles. On the basis of Penndorf's theoretical formulation of molecular scattering (Penndorf, 1957), Leckner (1978) has presented the approximate formula for the attenuation coefficient for the scattering by dry air at standard conditions

$$
k_{r \lambda}=0.008735 \cdot \lambda^{-4.08}
$$

with $\lambda$ in $\mu m$.

\section{B.2.2 Mie scattering of water vapour and dust particles}

Following Moon (1940), the coefficient for water vapour scattering (as referred in the literature) can be written as

$$
k_{w \lambda}=0.008635 \cdot \lambda^{-2}
$$


valid for $1.0 \mathrm{~cm}$ of precipitable water and optical path length of 1. It essentially treats water droplets.

On the other hand, the coefficient for dust scattering is as follow:

$$
k_{d \lambda}=0.08128 \cdot \lambda^{-.075}
$$

for 800 dust particles per $\mathrm{cm}^{3}$ and optical path length of 1 .

In Moon's coefficient for dust attenuation, the number of particles per unit volume can be varied. However, it is independent of the size of dust particles. Moreover, since attenuation effects due to scattering and absorption are difficult to separate, Ångström $(1929,1930)$ suggested a single formula

$$
k_{a \lambda}=\beta \cdot \lambda^{-\alpha}
$$

generally known as Ångström's turbidity formula. $\beta$ represents the amount of aerosols present in the atmosphere in the vertical direction. The wavelength exponent $\alpha$ is related to the size distribution of the aerosol particles. Large values indicate a relatively high ratio of small particles to large particles. It appears obvious that $\alpha$ varies from 4 (Rayleigh scattering) to 0 (extremely large particles).

\section{B.2.3 Absorption by gases}

While scattering by particles in the atmosphere and absorption of particular centers is a continuous function of the wavelength, absorption by gases occurs only at particular bands or wavelengths. It will only be considered here the absorption by ozone, since is the only one with attenuation coefficient different from 0 for the spectral ranges under consideration in this study. The attenuation coefficients for ozone for the wavelengths under study are shown in Table B.1.

\begin{tabular}{lllll}
\hline$\lambda(\AA)$ & 5303 & 5260 & 6374 & 6340 \\
\hline \hline$k_{o z}(\lambda)$ & 0.063 & 0.057 & 0.085 & 0.089 \\
\hline
\end{tabular}

Table B.1: Attenuation coefficient $\left(\mathrm{cm}^{-1}\right)$ for ozone for $l=1 \mathrm{~cm}$. Adapted from Iqbal (1983).

The amount of total ozone $l$ in a vertical column of air is given in units of atmosphere centimeters (atm $\mathrm{cm}$ ). This is the height of gaseous ozone if all the ozone in a vertical column of unit area were brought to normal temperature and surface pressure (NTP). Around the equator, total ozone averages $0.24 \mathrm{~cm}(\mathrm{NTP})$, the amount increasing with latitude. At higher latitudes the seasonal variation of ozone has to be kept in mind. The seasonal variation of the total amount of ozone $l$ at $30^{\circ} \mathrm{S}$, i.e., close to the latitude corresponding to El Leoncito Observatory $\left(31.8^{\circ} \mathrm{S}\right)$, is shown in Table B.2. 


\begin{tabular}{cccccccccccc}
\hline Jan & Feb & Mar & Apr & May & Jun & Jul & Aug & Sep & Oct & Nov & Dec \\
\hline \hline 0.27 & 0.28 & 0.26 & 0.27 & 0.28 & 0.28 & 0.29 & 0.31 & 0.32 & 0.32 & 0.29 & 0.29 \\
\hline
\end{tabular}

Table B.2: Seasonal variation of atmospheric ozone at $30^{\circ} \mathrm{S}$ latitude, in $\mathrm{cm}$ (NTP). Adapted from Iqbal (1983).

\section{B.3 Summary}

Having in mind the different attenuation coefficients, we are now able to write the transmittance of the Earth's atmosphere as

$$
\tau_{\lambda}=\tau_{r \lambda} \cdot \tau_{a \lambda} \cdot \tau_{o z \lambda}
$$

where

$$
\begin{gathered}
\tau_{r \lambda}=e^{-k_{r \lambda} X_{h} X\left(z_{t}\right)} \\
\tau_{a \lambda}=e^{-\beta \lambda^{\alpha} X_{h} X\left(z_{t}\right)} \\
\tau_{o z \lambda}=e^{-k_{o z \lambda} l X\left(z_{t}\right)}
\end{gathered}
$$

denote the transmission coefficients due to Rayleigh scattering, aerosols, and ozone, respectively. Note that the optical mass of ozone is not corrected for any variation of local pressure as it is the case for aerosols and Rayleigh. This is due to the fact that the ozone is mainly concentrated in a layer between 10 and $35 \mathrm{~km}$ altitude. Equations (B.2) and (B.6) allow to write

$$
I_{\lambda}=I_{0 \lambda} \cdot \tau_{\lambda}=I_{0 \lambda} \cdot e^{-K_{\lambda} \cdot X\left(z_{t}\right)}=I_{0 \lambda} \cdot e^{-\left(k_{r \lambda} X_{h}+\beta \lambda^{\alpha} X_{h}+k_{o z \lambda} l\right) \cdot X\left(z_{t}\right)}
$$

$K_{\lambda}$ being referred as the total extinction coefficient at the wavelength $\lambda$. 


\section{Appendix C}

\section{The HASTA Instrument}

HASTA $\left(\mathrm{H}_{\alpha}\right.$ solar telescope for Argentina, Bagalá et al., 1999) started operations on May, 1998 at the "GermanArgentinean Solar Observatory" in El Leoncito, San Juan, Argentina. It provides full solar disk images in the Hydrogen $\mathrm{H}_{\alpha}$ emission line (6563 $\AA$ ), tracing both solar magnetic activity and evolution of plages, filaments, prominences, sunspots, and flares. The technical specifications of the instrument are outlined in Table C.1 .

\begin{aligned} & \hline \hline Location: El Leoncito, San Juan, Argentina. \\ & Longitude $69.3^{\circ} \mathrm{W} \\ &$ Latitude $31.8^{\circ} \mathrm{S} \\ &$ Altitude $2400 \mathrm{~m} \\ &$\hline Objective: Observation of solar flares, plages and eruptive prominences. \\ & \hline Telescope: Diameter $=10 \mathrm{~cm}$; Focus $=170 \mathrm{~cm} \\ &$\hline Filter: Tunable Lyot-Filter \\ & Central Wavelength $6563 \AA \\ &$ Bandwidth $0.3 \AA \\ &$ Tuning range $\sim \pm 1 \AA \\ &$\hline Camera: CCD, $1280 \times 1024$ square pixels \\ & Pixel size $6.7 \mu m \times 6.7 \mu m \\ &$\hline Spatial Resolution: 1.5 arcsec \\ & \hline Operational Modes: Patrol mode (images every 2-3 min) \\ & High time resolution mode (images every $3 \mathrm{sec}$ ) \\ & \hline Integration Time: $50-100 \mathrm{msec} \\ &$\hline \hline\end{aligned}

Table C.1: $\mathrm{H}_{\alpha}$ solar telescope for Argentina (HASTA): Technical specifications. 


\section{List of Figures}

1.1 Image of a total solar eclipse $($ March 1988) $\ldots \ldots \ldots \ldots \ldots$

1.2 Relative intensity of the coronal light components . . . . . . . . . . . . . . . . 11

1.3 Panoramic view of "El Leoncito", San Juan, Argentina . . . . . . . . . . . . . . . . . . . 13

2.1 Optical scheme of a Lyot coronagraph . . . . . . . . . . . . . . . . . . . . . . . 17

2.2 Optical layout of the internally-occulted mirror coronagraph for Argentina (MICA) . . . . . . . 20

2.3 Photographs of the MICA telescope showing the filters box and camera shutter . . . . . . . . 24

2.4 Radial dependence of the central wavelength of the filter passband . . . . . . . . . . . . . . . . 24

2.5 Variation of the filter transmission with temperature and solar distance . . . . . . . . . . 25

2.6 Photographs of the MICA telescope showing auxiliary instruments . . . . . . . . . . . . . 32

2.7 Fine pointing mechanism: Optical layout . . . . . . . . . . . . . . . . . . . 33

2.8 The sky tester device as an externally-occulted coronagraph . . . . . . . . . . . . . 33

2.9 Examples of sky and Sun brightness intensity recorded with the sky and Sun tester devices . . . 35

2.10 Block diagram of the MICA system f . . . . . . . . . . . . . . . . . 37

2.11 Flow diagram showing default operation mode of the MICA telescope . . . . . . . . . . . . 39

2.12 Effect of the flying seeds of the Aspen trees in "El Leoncito" . . . . . . . . . . . . . . . . . . 40

2.13 Example of a Fe XIV coronal emission line image obtained with MICA on November 26, 1997 . . 41

2.14 Example of Fe XIV (green) and Fe X (red) coronal emission line images obtained with MICA on

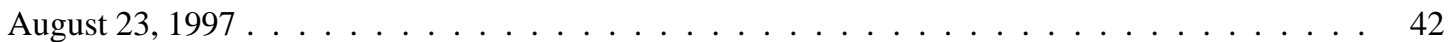

3.1 Sketch of the reduction procedure of MICA images: I. Ideal case . . . . . . . . . . . . . . . 49

3.2 Sketch of the reduction procedure of MICA images: II. Real case . . . . . . . . . . . . . . 50

3.3 Effect of the sky variability on MICA images . . . . . . . . . . . . . . . . . . 55

3.4 Two ways of computing the scaling factor $\beta \ldots \ldots \ldots \ldots$

3.5 Influence of the sky variability on the scaling factor $\beta \ldots \ldots \ldots \ldots$

3.6 Influence of changing air masses on the calibration . . . . . . . . . . . . . . . . . 60

3.7 Calculation of the normalization factor $f_{k} \ldots \ldots \ldots \ldots \ldots \ldots \ldots$

3.8 Comparison between calibrated green line coronal emission obtained with MICA and the Sacramento Peak coronagraph. I . . . . . . . . . . . . . . . . . . . . . . 66

3.9 Comparison between calibrated green line coronal emission obtained with MICA and the Sacramento Peak coronagraph. II . . . . . . . . . . . . . . . . . . . . . . . . . . . 67

4.1 Ionization equilibrium of $\mathrm{Fe}^{13+}$ and $\mathrm{Fe}^{9+}$ ions . . . . . . . . . . . . . . . . . . . . . 72

4.2 Typical green line structures $\ldots \ldots \ldots \ldots \ldots \ldots \ldots$ 
4.3 Sketch used for the characterization of coronal features . . . . . . . . . . . . . . 75

4.4 Composite of MICA and HASTA images on December 6, 1998 . . . . . . . . . . . . . . . 79

4.5 HASTA images of the NOAA AR 8340 on Sep. 23 and Sep. 27, 1998 . . . . . . . . . . . 83

4.6 Soft X-ray enhancement observed on September 30, 1998 by SXT/YOHKOH . . . . . . . . . . 83

4.7 GOES 10 plots on September 30, $1998 \ldots \ldots \ldots \ldots$. . . . . . . . . . . . . . 84

4.8 Combined observations (MICA and HASTA) of the September 30, 1998 event . . . . . . . . . 85

4.9 Signature of a continuum intensity enhancement in MICA images. I . . . . . . . . . . . . 86

4.10 Release of a "blob" on September 30, $1998 \ldots \ldots$. . . . . . . . . . . . . . . . . . 87

4.11 Height-time diagram of the "jet" and "blob" observed in the September 30, 1998 event . . . . . . 88

4.12 Signature of a continuum intensity enhancement in MICA images. II . . . . . . . . . . . . . 88

5.1 Building of the LASCO/C1 time series . . . . . . . . . . . . . . . . . . . 94

5.2 Typical LASCO/C1 time series and corresponding periodograms . . . . . . . . . . . . . . . 97

5.3 Synodic rotation period of the inner green line corona . . . . . . . . . . . . . . . 98

5.4 FWHM of the peaks denoting the computed synodic rotation periods . . . . . . . . . . . 98

5.5 Uncertainty of the period estimates with Method $\mathrm{B}_{0} \ldots \ldots \ldots \ldots$. . . . . . . . . . 99

5.6 Comparison between coronal and photospheric rotation rates . . . . . . . . . . . . . . 100

5.7 Residuals . . . . . . . . . . . . . . . . . . . . . . . . 101

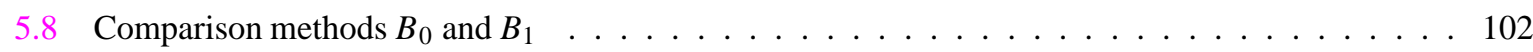

5.9 Example of the widening of the main peak of the periodograms . . . . . . . . . . . 103

5.10 Sample of time series used for harmonic filtering analysis . . . . . . . . . . . . . 106

5.11 Harmonic filtering results . . . . . . . . . . . . . . . . . . . . . 107

5.12 Periodograms obtained from synthetic time series . . . . . . . . . . . . . . . 109

5.13 Harmonic filtering applied to synthetic time series . . . . . . . . . . . . . . . . 110

5.14 DQCS coronal magnetic field model . . . . . . . . . . . . . . . . . . 112

5.15 Sample of EIT images (May 16, 1996) . . . . . . . . . . . . . . . . . . . . . . . 114

5.16 Definition of latitude strips ． . . . . . . . . . . . . . . . . . . 115

5.17 Latitude dependence of the synodic rotation rate as obtained from EIT images . . . . . . . . . 118

5.18 Synodic rotation period (rotation rate) as obtained from EIT data vs solar latitude. Comparison with photospheric rotation rates . . . . . . . . . . . . . . . . . . . . . . . 119

5.19 Distribution of the rotation rate estimates with Fe XII EIT data (April 1 to April 14, 1997) . . . . 121

5.20 Distribution of the rotation rate estimates with Fe XII EIT data (May 10 to May 23, 1996) . . . . . 122

A.1 Solar spectral irradiance . . . . . . . . . . . . . . . . . . . . . . . . . . 129

A.2 Solar spectral irradiance in the UV . . . . . . . . . . . . . . . . . . . . . . 130 


\section{List of Tables}

2.1 Filters: Technical specifications . . . . . . . . . . . . . . . . . . . . . 23

2.2 CCD Camera: Technical sheet . . . . . . . . . . . . . . . . . . . . . . 30

2.3 CCD Camera: Parameters obtained experimentally _ . . . . . . . . . . . . . . . 31

3.1 Experimental values obtained for the calibration factor $\xi_{i} \ldots \ldots \ldots \ldots$

5.1 Example to show disappearance of harmonics at $2 n T_{r o t}$ with Method $\mathrm{B}_{1} \quad \ldots \ldots$. . . . . . 103

5.2 Coefficients of the least square fit of the periods estimated with EIT data . . . . . . . . . . . . . 119

B.1 Spectral absorption coefficient for ozone . . . . . . . . . . . . . . . . . . 134

B.2 Seasonal variation of atmospheric ozone at $30^{\circ} \mathrm{S}$ latitude . . . . . . . . . . . . . . . . 135

C.1 HASTA telescope: Technical specifications . . . . . . . . . . . . . . . . . 136 



\section{Bibliography}

Allen, C.W., Astrophysical Quantities, 2nd Edition, (London: Athlone) (1963).

Ångström, A., "On the Atmospheric Transmission of Sun Radiation and on Dust in the Air", Geografis. Annal, 2, 156-166 (1929).

Ångström, A., “On the Atmospheric Transmission of Sun Radiation”, Geografis. Annal, 2 and 3, 130-159 (1930).

Antonucci, E., M. A. Dodero, "Rigid and Differential Rotation of the Solar Corona”, Solar Phys., 34, 3-10 (1974).

Antonucci, E., M. A. Dodero, "Rotation and Lifetime of Coronal Features”, Solar Phys., 62, 107-112 (1979).

Antonucci, E., L. .Svalgaard, “Green Corona and Solar Sector Structure”, Solar Phys., 36, 115-120 (1974).

Antonucci, E., R. Rosner, K. Tsinganos, “On Magnetic Field Stochasticity and Nonthermal Line Broadening in Solar Flares”, Solar Phys., 301, 975-980 (1986).

Arnaud, J., G. Newkirk, Jr., "Mean Properties of the Polarization of the Fe XIII 10747 Å Coronal Emission Line”, Astron. Astrophys., 178, 263-268 (1987).

Arnaud, J., R. Rothenflug, "An Updated Evaluation of Recombination and Ionization Rates”, Astron. Astrophys. Suppl. Ser., 60, 425-457 (1985).

Athay, R.G., G. E. Moreton, "Impulsive Phenomena of the Solar Atmosphere. I. Some Optical Events Associated with Flares Showing Explosive Phase", Astrophys. J., 133, 935-945 (1961).

Bagalá, L. G., O. H. Bauer, R. Fernandez Borda, C. Francile, G. Haerendel, R. Rieger, M. G. Rovira, “The New H-alpha Solar Telescope at the German-Argentinean Solar Observatory" in Proc. 9th European Meeting on Solar Physics, ESA-SP 448, 469-474 (1999).

Banaszkiewics, M., W. I. Axford, J. F. McKenzie, “An Analytic Solar Magnetic Field Model”, Astron. Astrophys., 337, 940-944 (1998).

Billings, D. E., S. Hirsch, C. Varsavsky, "Coronal Temperature Determination from Emission Lines”, Astrophys. J., 123, 532-535 (1956).

Bird, M. K., P. Edenhofer, "Remote Sensing Observations of the Solar Corona", in Physics and Chemistry in Space - Space and Solar Physics, Vol 20: Physics of the Inner Heliosphere (1. Large Scale Phenomena), Springer-Verlag, Berlin, 13-97 (1990).

Bonnet, R. M., L'Astronomie, 80, p. 191 (1966).

Born, M., Optik, Sringer-Verlag (1985).

Brueckner, G. E., R. A. Howard, M. J. Koomen, C. M. Korendyke, D. J. Michels, J. D. Moses, D. G. Socker, K. P. Dere, P. L. Lamy, A. Llebaria, M. V. Bout, R. Schwenn, G. M. Simnett, D. K. Bedford, C. J. Eyles, “The Large Angle Spectroscopic Coronagraph (LASCO)”, Solar Phys., 162, 357-402 (1995).

Burgess, A., M. Seaton, “The Ionization Equilibrium for Iron in the Solar Corona”, M.N.R.A.S, 127, 355-358 (1964). 
Carrington, R. C., "Observations of the Spots on the Sun”, Williams and Norgate, London (1863).

Chapman, S., Smithsonian Contr. Astrophys, 2, p. 1 (1957).

Chitre, S. M., "Theory of Umbral Oscillations and Penumbral Waves", in Sunspots: Theory and Observations. Proceedings of the NATO Advanced Research Workshop on the Theory of Sunspots, Cambridge, United Kingdom, 333-343 (1991).

Cooper, R. H., D. E. Billings, “A Long-Lived Polar Coronal Region”, Z. Astrophys., 55, $24-28$ (1962).

Davies, S. R., “An Improved Test for Periodicity”, M.N.R.A.S, 244, $93-95$ (1990).

Delaboudinière, J.-P., G. E. Artzner, J. Brunaud, A. H. Gabriel, J. F. Hochedez, F. Millier, X. Y. Song, B. Au, K. P. Dere, R. A. Howard, R. Kreplin, D. J. Michels, J. D. Moses, J. M. Defise, C. Jamar, P. Rochus, J. P. Chauvineau, J. P. Marioge, R. C. Catura, J. R. Lemen, L. Shing, R. A. Stern, J. B. Gurman, W. M. Neupert, A. Maucherat, F. Clette, P. Cugnon, E. L. Van Dessel, "EIT: Extreme-Ultraviolet Imaging Telescope for the SOHO Mission", Solar Phys., 162, 291-312 (1995).

Dunn, R. B., "Coronal Events Observed in 5303 ^”, in Physics of the Solar Corona. ed. by C. Macris, D. Reidel Publishing Company, Dordrecht-Holland, 114-129 (1971).

Dupree, A. K., C. E. Huber, “The Extreme-Ultraviolet Spectrum of a Solar Active Region”, Astrophys. J., 182, 321-333 (1973).

Edlén, B., "Mg I-ähnliche Spektren der Elemente Titan bis Cobalt, Ti XI, V XII, Cr XIII, Mn XIV, Fe XV und Co XVI", Z. Phys., 103, 536-541 (1936).

Edlén, B., "S I-ähnliche Spektren der Elemente Titan bis Eisen, Ti VII, V VIII, Cr IX, Mn X und Fe XI", Z. Phys., 104, 188-193 (1937).

Edlén, B., "Die Deutung der Emissionslinien im Spektrum der Sonnenkorona”, Z. Astrophys., 22, 30-64 (1942).

Epple, A., R. Schwenn, "PICO - A Mirror Coronagraph on Pic Du Midi", in Proc. Third SOHO Workshop, Estes Park, Co., ESA-SP 373, 399-402 (1994).

Epple, A., "Erdgebundene Beobachtungen der Sonnenkorona mit einem Spiegelkoronagraphen", PhD Thesis, Georg-Augusta-Universität zu Göttingen (1997).

Esser, R., N. S. Brickhouse, S. R. Habbal, R. C. Altrock, H. S. Hudson, "Using Fe X $6374 \AA$ A and Fe XIV $5303 \AA$ Spectral Line Intensities to Study the Effect of Line of Sight Integration on Coronal Temperature Inferences”, Journ. Geophys. Res., 100, 19829-19838 (1995).

Evans, J. W., "A Photometer for Measurement of Sky Brightness Near the Sun”, J. Opt. Soc. A., 38, 1083-1085 (1948).

Evans, J. W., "The Coronagraph" in The Sun, ed. G. P. Kuiper, University of Chicago Press, Chicago, (1953).

Ferraz-Mello, S. "Estimation of Periods from Unequally Spaced Observations", Astron. J., 86(4), 619-624 (1981).

Fisher, R., AFCRL Instrumentation Paper 205, NTIS No. AD775745 (1973).

Fisher, R., C. Garcia, "Detection of a Slowly Moving Coronal Transient Event", Astrophys. J. Letters, 282, L35L37 (1984). 
Forbes, T. G., E. R. Priest, "Photospheric Magnetic Field Evolution and Eruptive Flares”, Astrophys. J., 446, 377-389 (1995).

Foukal, P., Solar Astrophysics, ed. by John Wiley \& Sons Inc., New York (1990).

Gilman, P. A., R. Howard, "Variations in Solar Rotation with the Sunspot Cycle", Astrophys. J., 283, 385-391 (1984).

Gilman, P. A., R. Howard, "Rotation Rates of Leader and Follower Sunspots ”, Astrophys. J., 295, 233-240 (1985).

Giovanelli, R. G., “Oscillations and Waves in a Sunspot”, Solar Phys., 27, 71-79 (1972).

Gosling, J. T., “The Solar Flare Myth”, Journ. Geophys. Res., 98, 18937-18949 (1993).

Gosling, J. T., E. Hildner, R. M. MacQueen, R. H. Munro, A. I. Poland, C. L. Ross, "Mass Ejections from the Sun: A View from Skylab”, Journ. Geophys. Res., 79, 4581-4587 (1974).

Grotrian, W., "Zur Frage der Deutung der Linien im Spektrum der Sonnenkorona”, Die Naturwissenschaften, 27, 214-214 (1939).

Guhathakurta, M., R. R. Fisher, R. C. Altrock, "Large-Scale Coronal Temperature and Density Distributions, 1984-1992”, Astrophys. J. Letters, 414, L145-L148 (1993).

Guhathakurta, M., R. R. Fisher, "Latitudinal Variability of Large-Scale Coronal Temperature and its Association with the Density and the Global Magnetic Field", Solar Phys., 152, 181-188 (1994).

Hoeksema, J. T., "The Relationship of the Large-Scale Solar Field to the Interplanetary Magnetic Field: What will Ulysses find?", in The Sun and the Heliosphere in Three Dimensions, ed. by R. G. Marsden, D. Reidel Publ. Co., Dordrecht (Holland), 241-254 (1986).

Horne, J. H., S. L. Baliunas, “A Prescription for Period Analysis of Unevenly Sampled Time Series”, Astrophys. J., 302, 757-763 (1986).

Howard, R., J. M. Adkins, J. E. Boyden, T. A. Cragg, T. S. Gregory, B. J. LaBonte, S. P. Padilla, L. Webster, “Solar Rotation Results at Mount Wilson”, Solar Phys., 83, 321-338 (1983).

Howard, R., P. A. Gilman, P. I. Gilman, "Rotation of the Sun Measured from Mount Wilson White-Light Images”, Astrophys. J., 283, 373-384 (1984).

Howard, R. A., N. R. Sheeley Jr., M. J. Koomen, D. J. Michels, “Coronal mass ejections: 1979-1981”, Journ. Geophys. Res., 90, 8173-8191 (1985).

Huber, M. C. E., A. K. Dupree, L. Goldberg, R. W. Noyes, W. H. Parkinson, E. M. Reeves, G. L. Withbroe, “The Harvard Experiment on OSO-6: Instrumentation, Calibration, Operation, and Description of Observations", Astrophys. J., 183, 291-312 (1973).

Hundhausen, A. J., "Flare-Produced Interplanetary Shock Waves", in Coronal Expansion and Solar Wind, Springer-Verlag, 169-207 (1972).

Hundhausen, A. J., C. B. Sawyer, L. House, R. M. E. Illing, W. J. Wagner, “Coronal Mass Ejections Observed During the Solar Maximum Mission: Latitude Distribution and the Rate of Occurrence”, Journ. Geophys. Res., 89, 2639-2646 (1984). 
Hundhausen, A., "Coronal Mass Ejections", in The Many Faces of the Sun: A Summary of the Results from NASA’s Solar Maximum Mission, Springer-Verlag New York, Inc., 143-200 (1999).

Inhester, B., G. Stenborg, R. Schwenn, N. Srivastava, B. Podlipnik, "The Rotation of the Fe XIV Solar Corona During the Recent Solar Activity Minimum”, Space Sci. Rev., 87, 211-214 (1999).

Innes, D. E., W. Curdt, D. E. McKenzie, R. Schwenn, S. Solanki, G. Stenborg, "Direct Evidence for an Explosive Start to Solar Mass Ejection”, Submitted for publication (2000).

Iqbal, M., An Introduction to Solar Radiation, Academic Press Canada, (1983).

Janiseck, J. R., "Scientific Charge-Coupled Devices", Short Course Notes, S.P.I.E. Symposium on Electronic Imaging, San Jose, S.P.I.E. SC- 22 (1991).

Kohl, J. L., E. M. Reeves, B. Kirkham, “New Instrumentation for Space Astronomy”, eds. K. A. van der Hucht and G. Vaiana, New York: Pergamon, p. 91 (1978).

Koomen, M. J., C. R. Detwiler, G. E. Brueckner, H. W Cooper, R. Tousey, "White Light Coronagraph in OSO-7”, Appl. Optics, 14, 743-751 (1975).

Kosovichev, A. G., J. Schou, P. H. Scherrer, R. S. Bogart, R. I. Bush, J. T. Hoeksema, J. Aloise, L. Bacon, A. Burnette, C. De Forest, P. M. Giles, K. Leibrand, R. Nigam, M. Rubin, K. Scott, S. D. Williams, Sarbani Basu, Christensen-Dalsgaard, W. Dappen, E. J. Rhodes Jr., T. L. Duvall Jr., R. Howe, M. J. Thompson, D. O. Gough, T. Sekii, J. Toomre, T. D. Tarbell, A. M. Title, D. Mathur, M. Morrison, J. L. R. Saba, C. J. Wolfson, I. Zayer, P. N. Milford, "Structure and Rotation of the Solar Interior: Initial Results from the MDI Medium-1 Program”, Solar Phys., 170, 43-61 (1997).

Kosovichev, A. G., V. V. Zharkova, "Observation of Seismic Effects of Solar Flares from SOHO Michelson Doppler Imager", in New Eyes to See Inside the Sun and Stars, ed. by Franz-Ludwig Deubner, Joergen Christensen-Dalsgaard, and Don Kurtz., IAUS, 185, p. 191 (1998).

Koutchmy, S., "Short Period Coronal Oscillations: Observation and Interpretation”, Astron. Astrophys., 120, 185191 (1983).

Koutchmy, S., “Space-Borne Coronagraphy”, Space Sci. Rev., 47, 95-143 (1988).

Leckner, B., "The Spectral Distribution of Solar Radiation at the Earth's Surface - Elements of a Model”, Sol. Energy, 20(2), 143-150 (1978).

Leibacher, J. W., R. W. Noyes, J. Toomre, R. K. Ulrich, “Helioseismology”, Sci. Am., 253, 48-57 (1985).

Lewis, D. J., G. M. Simnett, G. E. Brueckner, R. A. Howard, P. L. Lamy, R. Schwenn, "LASCO Observations of the Coronal Rotation", Solar Phys., 184, 297-315 (1999).

Lomb, N. R., "Least-Squares Frequency Analysis of Unequally Spaced Data”, Ap\&SS, 39, 447-462 (1976).

Low, B. C., "Solar Activity and the Corona", Solar Phys., 167, 217-265 (1996).

Lyot, B., Compt. Rend. Acad. Sci., 193, p. 1169 (1931).

Lyot, B., "A Study of the Solar Corona and Prominences Without Eclipses", M.N.R.A.S, 99, 580-595 (1939).

Mackay, C. D., “Charge-coupled devices in astronomy”, Ann. Rev. Astron. Astrophys., 24, 255-283 (1986). 
MacQueen, R. M., R. R. Fisher, “The Kinematics of Solar Coronal Transients”, Solar Phys., 89, 89-102 (1983).

Magara, T., K. Shibata, "Plasmoid Formation in Eruptive Flares”, Adv. Space Res., 19, 1903-1906 (1997).

Mc Lean, I. S., Electronic and Computer-aided Astronomy, Chichester: Ellis Horwood limited, John Wiley and Sons (1989).

Mie, G., Ann. Physik (4), 25 (1908).

Moon, P., "Proposed Standard Solar Radiation Curves for Engineering Use”, J. Franklin Inst., 230, 583-617 (1940).

Moreton, G. E., "Fast-Moving Disturbances on the Sun”, Sky \& Telescope, 21, p. 145 (1961).

Moses, D., F. Clette, J.-P. Delaboudinière, G. E. Artzner, M. Bougnet, J. Brunaud, C. Carabetian, A. H. Gabriel, J. F. Hochedez, F. Millier, X. Y. Song, B. Au, K. P. Dere, R. A. Howard, R. Kreplin, D. J. Michels, J. M. Defise, C. Jamar, P. Rochus, J. P. Chauvineau, J. P. Marioge, R. C. Catura, J. R. Lemen, L. Shing, R. A. Stern, J. B. Gurman, W. M. Neupert, J. Newmark, B. Thompson, A. Maucherat, F. Portier-Fozzani, D. Berghmans, P. Cugnon, E. L. Van Dessel, J. R. Gabryl, "EIT Observations of the Extreme Ultraviolet Sun”, Solar Phys., 175, 571-599 (1997).

Newberry, M. V., “Signal-to-Noise Considerations for Sky-Subracted CCD Data”, Publ. Astron. Soc. Pacific, 103, 122-130 (1991).

Newkirk, G., D. Bohlin, "Reduction of Scattered Light in the Coronagraph”, Appl. Optics, 2, 131-140 (1963).

Newton, H. W., M. L. Nunn, “The Sun's Rotation Derived from Sunspots 1934-1944 and Additional Results”, M.N.R.A.S, 111, 413-421 (1951).

Newton, E. K., A. G. Emslie, J. T. Mariska, “The Velocity Differential Emission Measure: Diagnostic of Bulk Plasma Motion in Solar Flares”, Astrophys. J., 447, 915-922 (1995).

Noëns, J. C., J. Pageault, G. Ratier, "Measuring Electron Density in Coronal Active Regions. II. A Multichannel Coronagraph with a Photoelectric Spectrograph and a Reflex Monitor at $\lambda 5303$ Å”, Solar Phys., 94, 117-131 (1984).

Parker, E. N., "Dynamics of the Interplanetary Gas and Magnetic Fields”, Astrophys. J., 128, 664-676 (1958).

Patty, S. R., M. J. Hagyard, "Delta-Configurations - Flare Activity and Magnetic-Field Structure”, Solar Phys., 103, 111-128 (1986).

Pecker, J. C., D. E. Billings, W. O. Roberts, “Identification of the Yellow Coronal Line”, Astrophys. J., 120, 509-520 (1954).

Penndorf, R., "Tables of the Refractive Index for Standard Air and the Rayleigh Scattering Coefficient for the Spectral Region between 0.2 and $20 \mu m$ and Their Application to Atmospheric Optics”, J. Opt. Soc. A., 47(2), 176-182 (1957).

Prabhu, T. P., Y. D. Mayya, G. C. Anupama, “Gain Calibration of CCD Systems at VBO”, J. Astrophys. Astr., 13, 129-144 (1992).

Raju, K. P., J. Singh, “Comparison of Computed Fluxes for Fe X and Fe XIV Lines with Observed Values at 1980 Eclipse”, Solar Phys., 110, 271-280 (1987). 
Raju, K. P., J. N. Desai, T. Chandrasekhar, N. M. Ashok, “The Excitation Mechanism of Fe XIV 5303 Å Forbidden Line in the Inner Regions of Solar Corona”, J. Astrophys. Astr., 12, 311-317 (1991).

Raju, K. P., J. N. Desai, “The Excitation Mechanism and the Line-to-Continuum Intensity Ratio of the [Fe XIV] 5303 Å Line in Various Coronal Regions”, Solar Phys., 147, 255-261 (1993).

Ramsey, H. E., S. F. Smith, “Flare-Initiated Filament Oscillations”, Astron. J., 71, 197-199 (1966).

Roesler, F. L., "Fabry-Perot Instruments for Astronomy”, in Methods of Experimental Physics 12: Astrophysics, Part A: Optical and Infrared, edited by N. Carleton, Academic Press Inc. (London) Ltd., 531-569 (1974).

Roberts, D. A., M. L. Goldstein, "Evidence for a High-Latitude Origin of Lower Latitude High-Speed Wind", Geophys. Res. Letters, 25, 595-598 (1998).

Scargle, J. D., "Studies in Astronomical Time Series Analysis II: Statistical Aspects of Spectral Analysis of Unevenly Spaced Data”, Astrophys. J., 263, 835-853 (1982).

Sheeley Jr., N. R., Y.-M. Wang, S. H. Hawley, G. E. Brueckner, K. P. Dere, R. A. Howard, M. J. Koomen, C. M. Korendyke, D. J. Michels, S. E. Paswaters, D. G. Socker, O. C. St Cyr, D. Wang, P. L. Lamy, A. Llebaria, R. Schwenn, G. M. Simnett, S. Plunket, D. A. Biesecker "Measurements of Flow Speeds in the Corona between 2 and $30 \mathrm{R}_{\odot} "$, Astrophys. J., 484, 472-478 (1997).

Scheiner, C., Rosa Ursini sive solis: Book 4, Part 2 (1630).

Scherrer, P. H., R. S. Bogart, R. I. Bush, J. T. Hoeksema, A. G. Kosovichev, J. Schou, W. Rosenberg, L. Springer, T. D. Tarbell, A. Title, C. J. Wolfson, I. Zayer, and the MDI engineering team, "The Solar Oscillation Investigation - Michelson Doppler Imager”, Solar Phys., 162, 129-188 (1995).

Schwarzenberg-Czerny, A., "On the Advantage of Using Analysis of Variance for Period Search", M.N.R.A.S, 241, 153-165 (1989).

Schwarzenberg-Czerny, A., "The Correct Probability Distribution for the Phase Dispersion Minimization Periodogram", Astrophys. J., 489, 941-945 (1997).

Schwenn, R., "Direct Correlations Between Coronal Transients and Interplanetary Disturbances”, Space Sci. Rev., 34, 85-99 (1983).

Schwenn, R., "Relationship of Coronal Transients to Interplanetary Shcks: 3D Aspects", Space Sci. Rev., 44, 139-168 (1986).

Schwenn, R., B. Inhester, S. P. Plunkett, A. Epple, B. Podlipnik, D. K. Bedford, C. J. Eyles, G. M. Simnett, S. J. Tappin, M. V. Bout, P. L. Lamy, A. Llebaria, G. E. Brueckener, K. P. Dere, R. A. Howard, M. J. Koomen, C. M. Korendyke, D. J. Michels, J. D. Moses, N. E. Moulton, S. E. Paswaters, D. G. Socker, O. C. St.Cyr, D. Wang, "First View of the Extended Green-Line Emission Corona at Solar Activity Minimum Using the LASCO-C1 Coronagraph on SOHO”, Solar Phys., 175, 667-684 (1997).

Shibata, K., Y. Ishido, L. W. Acton, K. T. Strong, T. Hirayama, Y. Uchida, A. H. McAllister, R. Matsumoto, S. Tsuneta, T. Shimizu, H. Hara, T. Sakurai, K. Ichimoto, Y. Nishino, Y. Ogawara, "Observations of X-Ray Jets with the YOHKOH Soft X-Ray Telescope”, PASJ, 44, L173-L179 (1992). 
Shklovskii, I. S., Physics of the Solar Corona, Moscow: Fizmatgiz; translated 1965, Oxford: Pergamon Press, (1962).

Sime, D. G., R. R. Fisher, R. C. Altrock, "Rotation Characteristics of the Fe XIV (5303 Å) Solar Corona", Astrophys. J., 336, 454-467 (1989).

Smartt, R. N., "Special Applications of the Point-Diffraction Interferometer", in Interferometry, S.P.I.E. Proceedings, 192, 35-40 (1979).

Smartt, R. N., "Solar Corona Photoelectric Photometer Using Mica Etalons", in Instrumentation in Astronomy IV, S.P.I.E. Proceedings, 331, 442-447 (1982).

Smartt, R. N., S. L. Koutchmy, S. A. Colley, R. Caron, R. Schwenn, S. R. Restaino, "New Technology Mirror Coronagraph with Extended Applications”, in Advanced Technology Optical Telescopes IV, S.P.I.E. Proceedings, 1236, 206-214 (1990).

Smith, S. F., K. L. Harvey, "Observational Effects of Flare-Associated Waves", in Physics of the Solar Corona, ed. by C. Macris, D. Reidel Publishing Company, Dordrecht-Holland, 156-167 (1971).

Snodgrass, H. B., “Magnetic Rotation of the Solar Photosphere”, Astrophys. J., 270, 288-299 (1983).

Snodgrass, H. B., R. K. Ulrich, "Rotation of Doppler Features in the Solar Photosphere", Astrophys. J., 351, 309-316 (1990).

Srivastava, N., R. Schwenn, B. Inhester, G. Stenborg, B. Podlipnik, "Measurements of Flow Speeds and Acceleration in Gradually Evolving Solar Mass Ejections as Observed by LASCO" in Solar Wind Nine, AIP CP 471, 115-118 (1999).

Starck, J.-L., R. Siebenmorgen, R. Gredel, "Spectral Analysis using the Wavelet Transform”, Astrophys. J., 482, 1011-1020 (1997).

Steinolfson, R. S., J. M. Davila, "Coronal Heating by the Resonant Absorption of Alfvén Waves - Importance of the Global Mode and Scaling Laws", Astrophys. J., 415, 354-363 (1993).

Stellingwerf, R. F., "Period Determination Using Phase Dispersion Minimization”, Astrophys. J., 224, 953-960 (1978).

Stenborg, G., R. Schwenn, N. Srivastava, B. Inhester, B. Podlipnik, M. Rovira, C. Francile, "MICA: The Mirror Coronagraph for Argentina", Space Sci. Rev., 87, 307-310 (1999a).

Stenborg, G., R. Schwenn, N. Srivastava, B. Inhester, B. Podlipnik, M. Rovira, C. Francile, "Recent Observations of the Solar Corona with a New Ground-Based Coronagraph in Argentina (MICA)" in Solar Wind Nine, AIP CP-471, 561-564 (1999b).

Stenborg, G., L. G. Bagal, O. H. Bauer, R. Fernndez Borda, C. Francile, G. Haerendel, M. G. Rovira, R. Schwenn, "First Combined Observations in the German-Argentinean Solar Observatory: Correlations in Quiet and Eruptive Phenomena at the Limb", JASTP, in Press (2000).

Stenflo, J. O., "Differential Rotation of the Sun's Magnetic Field Pattern", Astron. Astrophys., 210, 403-409 (1989). 
Strong, K. T., K. Harvey, T. Hirayama, N. Nitta, T. Shimizu, S. Tsuneta, “Observations of the Variability of Coronal Bright Points by the Soft X-Ray Telescope on YOHKOH”, PASJ, 44, L161-L166 (1992).

Tandberg-Hansen, E., The Nature of Solar Prominences, ed. by Kluwer Academic Publishers, Dordrecht (Holland) (1995).

Ternullo, M., "Sunspots: Their Rotation, Their Expansion in the Activity Zone, Their Links with the Giant Convective Cells", Solar Phys., 127, 29-50 (1990).

Thompson, B. J., J. B. Gurman, W. M. Neupert, J. S. Newmark, J.-P. Delaboudiniere, O. C. St. Cyr, S. Stezelberger, K. P. Dere, R. A. Howard, D. J. Michels, "SOHO/EIT Observations of the 1997 April 7 Coronal Transient: Possible Evidence of Coronal Moreton Waves”, Astrophys. J., 517, L151-L154 (1999).

Tousey, R., “The Solar Corona”, Adv. Space Res., 13, 713-730 (1973).

Trellis,, M., “Contribution à l'etude de la Couronne Solaire”, Ann. Astrophys. Suppl. Ser., fasc. 5 (1957).

Tsuneta, S., L. Acton, M. Bruner, J. Lemen, W. Brown, R. Caravalho, R. Catura, S. Freeland, B. Jurcevich, J. Owens, “The Soft X-Ray Telescope for the SOLAR-A Mission”, Solar Phys., 136, 37-67 (1991).

van der Hulst, H. C., "The Chromosphere and the Corona” in The Sun, ed. G. P. Kuiper, University of Chicago Press, Chicago, (1953).

Volz, F., "Die Optik und Meteorologie der atmosphärischen Trübung”, Ber. des Dt. Wetterd., Nr. 13, Band 2, Bad Kissingen (1954).

Waldmeier, M., "Monochromatische Photometrie der Sonnenkorona”, Z. Astrophys., 22, 1-17 (1942).

Waldmeier, M., "Polarkarten der Sonnenkorona”, Z. Astrophys., 27, 24-41 (1950).

Waldmeier, M., "The Structure of the Monochromatc Corona in the Surroundings of Prominences", Solar Phys., 15, 167-175 (1970).

Wang, Y.-M., N. R. Shelley Jr., A. G. Nash, L. R. Shampine, “The Quasy-Rigid Rotation of Coronal Magnetic Fields", Astrophys. J., 327, 427-450 (1988).

Wang, Y.-M., N. R. Shelley Jr., “The Rotation of Photospheric Magnetic Fields: A Random Walk Transport Model", Astrophys. J., 430, 399-412 (1994).

Withbroe, G. L., W. C. Feldman, H. S. Ahluwalia, “The Solar Wind and Its Coronal Origins”, in Solar Interior and Atmosphere, ed. by A. N. Cox, W. C. Livingston, and M. S. Matthews, University of Arizona Press, Tucson, 1087-1106 (1991).

Young, A. T., W. M. Irvine, "Multicolor Photoelectric Photometry of Brighter Planets. I. Program and Procedure", Astron. J., 72, 945-950 (1967).

Zirin, H., A. Stein, “Observations of Running Penumbral Waves”, Astrophys. J. Letters, 178, L85-L87 (1972).

Zirin, H., Astrophysics of the Sun, Cambridge University Press, New York, (1988). 


\section{Danksagung}

Die vorliegende Arbeit wurde am Max-Planck-Institut für Aeronomie (MPAe) in Katlenburg-Lindau angefertigt. Der Max-Planck-Gesellschaft und den Direktoren des Instituts möchte ich für die Gewährung des Stipendiums und für die Bereitstellung des gut ausgestatteten Arbeitsplatzes danken. Auch für die Möglichkeit der Teilnahme an internationalen Konferenzen bedanke ich mich.

Für die Betreuung dieser Arbeit danke ich Herrn Prof. Dr. R. Schwenn. Seine Begeisterung für die Sonnenphysik und sein Interesse am wissenschaftlichen Fortgang, sowie seine freundliche Unterstützung halfen mir über manches Problem hinweg. Herrn Prof. Dr. F. Kneer danke ich für die Übernahme der Betreuung meiner Arbeit an der Universität Göttingen und die besondere Hilfestellung in einer für mich zunächst fremden Umgebung.

Mein ganz besonderer Dank gilt Herrn Dr. A. Epple, der mich bei meinen ersten Schritten in der Koronagraphie begleitete. Auf Grund seiner Kenntnisse im Bereich der Sonnenbeobachtung bekam ich eine einfassende Einführung in die Funktion des Gerätes und die Methoden der Bildverarbeitung.

Den Herren R. Schmidt, U. Strohmeyer und W. Neumann sei an dieser Stelle für die gute und angenehme Zusammenarbeit gedankt, sowie allen Mitarbeitern des MPAe, die am MICA-Projekt mitgearbeitet haben.

Mein ganz besonderer Dank gilt Herrn C. Francile für seine große Hilfe bei der Lösung von technischen Problemen und notwendigen Reparaturen des MICA-Teleskops, sowie für seine liebe Gastfreundschaft während meiner Besuche in San Juan. Die Herren A. Buenaventura Cornudella, A. A. Gonzalez, W. G. Gomez und C. Francile sorgen für den ganzjährigen Betrieb des MICA-Teleskops. Hierfür möchte ich ihnen danken. Ebenso möchte ich mich bei der Belegschaft des "OAFA Observatorium in El Leoncito" herzlich für die vielen schönen Tage in ihrer Mitte bedanken.

Danken möchte ich auch Frau Dr. N. Srivastava für das Korrekturlesen des Manuskripts, die zahllosen fachlichen Diskussionen und die daraus resultierenden Verbesserungsvorschläge. Ihre Hilfbereitschaft und ihre Freundlichkeit auch über das Fachliche hinaus waren eine schöne Erfahrung für mich. Weiterhin danke ich Herrn Dr. B. Inhester für die vielen erhellenden Gespräche zur Sonnenrotation, sowie für seine Einsatzfreude bei allen sonstigen Fragen. Frau Dr. G. Bagalá möchte ich für die Hilfe bei den HASTA-Beobachtungen und für ihren freundschaftlichen Rat danken. Herrn Dr. R. Altrock von Sacramento Peak Observatory in der Vereinigten Staaten danke ich für die Überlassung der Daten, die ich für den Vergleich mit MICA-Daten verwendete.

Frau Dr. M. Rovira, die bereits meine Diplomarbeit betreut hat, möchte ich dafür danken, daß sie auch weiterhin eine Ansprechpartnerin für mich geblieben ist und immer wieder Anteil an meiner Arbeit genommen hat.

Den Mitarbeitern des Rechenzentrums des MPAe und besonders herzlich Herrn B. Podlipnik möchte ich für ihre Unterstützung danken.

Besonderen Dank verdienen schließlich meine Frau, Marta Carolina Sarmiento de Stenborg, und meine Tochter, María Sofía, für die geduldige Unterstützung während der Arbeit und ihr Verständnis für die vielen Abende und Wochenenden meiner Abwesenheit in Rahmen dieser Arbeit. 



\section{Lebenslauf}

Am 01. Januar 1963 wurde ich als Sohn des Ehepaars Sten Lars Stenborg und Alicia Martha D'Alessandro in Buenos Aires, Argentinien, geboren.

Von 1969 bis 1975 besuchte ich die dortige Grundschule "Colegio Marianista". Von 1976 bis 1980 besuchte ich das Gymnasium "Liceo Militar General San Martin” in Buenos Aires.

Anfang 1981 immatrikulierte ich mich an der Universität "Instituto Tecnológico Buenos Aires" (ITBA), wo ich die ersten zwei Jahre Elektronik studierte.

Im Rahmen einer Ausleseprüfung bekam ich 1983 ein Stipendium für ein Physikstudium an der "Instituto Balseiro" (Universität Nacional de Cuyo, Bariloche, Argentinien) zugesprochen und setzte dort mein Studium fort. Aus persönlichen Gründen ging nach einem Semester zurück nach Buenos Aires.

1984 setzte ich mein Studium an der "Facultad de Ciencias Exactas Y Naturales" an der Universität Buenos Aires (UBA) fort. Meine Diplomarbeit fertigte ich am "Instituto de Astronomía y Física del Espacio" (IAFE) in Buenos Aires unter der Leitung von Dr. Marta Rovira an. Das Thema der Arbeit lautete: "Cálculo de Espectros Metálicos en Protuberancias Solares". Im Dezember 1994 bekam ich meinen Abschluß "Licenciatura en Ciencias Físicas". Das entspricht einem Diplom in Physik in Deutschland und wurde hier auch so anerkannt.

Am 18. Marz 1988 heiratete ich die Architektin Marta Carolina Sarmiento. Am 23. Oktober 1990 wurde unsere Tochter María Sofía geboren.

Im August 1995 wechselte ich zum Max-Planck-Institut für Aeronomie in Katlenburg-Lindau (Deutschland). Dort arbeitete ich von August 1995 bis Mai 1996 and von November 1996 bis Januar 1997 als wissenschaftlicher Mitarbeiter an der Entwicklung der Software für das MICA-Projekt.

Im Juni 1997 bekam ich ein Doktoranden-Stipendium am Max-Planck-Institut für Aeronomie. Von Juni 1997 bis Dezember 1997 arbeitete ich an der Installation des MICA-Teleskops, am German-Argentinien Solar Observatorium im "Estación de Altura Prof. Ulrico Cesco", das eine Außenstelle vom "Observatorio Astronómico Félix Aguilar" (OAFA) in El Leoncito (San Juan, Argentinien) ist. Zusätzlich war ich verantwortlich für die Kalibrierung des Teleskops und für die Feinabstimmung der Software. Danach zog ich nach Deutschland um, wo ich an der vorliegenden Dissertation arbeitete. Zum Sommersemester 1998 immatrikulierte ich mich an der GeorgAugust-Universität in Göttingen. 